\title{
п्.
}

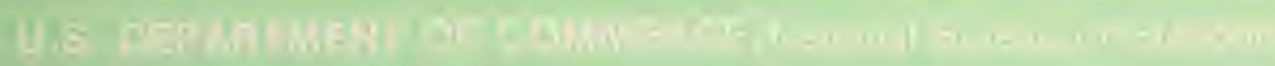




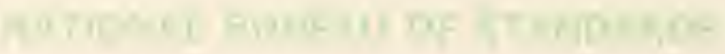

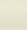

r 


\section{SPEED2, A Computer Program for the Reduction of Data from Automatic Data Acquisition Systems}

Richard D. Peacock

Center for Fire Research

National Engineering Laboratory

National Bureau of Standards

Washington, DC 20234

and

John M. Smith

Institute for Computer Sciences and Technology

National Bureau of Standards

Washington, DC 20234

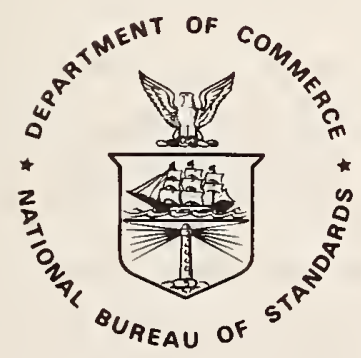

U.S. DEPARTMENT OF COMMERCE, Juanita M. Kreps, Secretary

Luther H. Hodges, Jr., Under Secretary

Jordan J. Baruch, Assistant Secretary for Science and Technology

NATIONAL BUREAU OF STANDARDS, Ernest Ambler, Director

Issued September 1979 


\section{National Bureau of Standards Technical Note 1108}

Nat. Bur. Stand. (U.S.), Tech. Note 1108, 153 pages (Sept. 1979)

CODEN: NBTNAE 
1. INTRODUCTION

2. SPEED2, PURPOSE AND STRUCTURE . . . . . . . . . . . . . . . . . 3

2.1 Purpose of SPEED2 . . . . . . . . . . . . . . . . . . . 3

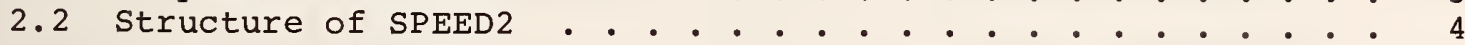

3. MAIN PROGRAM, SPEED2 . . . . . . . . . . . . . . . . . . . 5

4. PLOT1, PURPOSE AND STRUCTURE . . . . . . . . . . . . . . . . 5

5. PLOT2, PURPOSE AND STRUCTURE . . . . . . . . . . . . . . . . . . 6

5.1 Using PLOT2 . . . . . . . . . . . . . . . . . . . . 7

5.2 Data Transformation . . . . . . . . . . . . . . . . . . . . . 7

5.2 .1 Linear Transformations . . . . . . . . . . . . . 7

5.2.2 More Complex Transformations . . . . . . . . . 8

5.3 Making Corrections to the Data Matrix . . . . . . . . . . 10

5.4 Generating Graphs . . . . . . . . . . . . . . . . . . 11

6. PLOT3, CALCOMP PEN PLOTTING OF DATA . . . . . . . . . . . . . . 12

6.1 Using PLOT3 . . . . . . . . . . . . . . . . . . . . . 13

6.2 Generating Pen Plots. . . . . . . . . . . . . . . . 13

7. PLOT4, COMBINING SEVERAL SETS OF DATA . . . . . . . . . . . . . 15

7.1 Using PLOT4 •. . . . . . . . . . . . . . . . . . . . . 15

7.2 Renumbering of Instruments Using PLOT4 . . . . . . . . . . . . 16

8. RESTRICTIONS ON THE USE OF SPEED2 . . . . . . . . . . . . . . . . 16

9. NOTES ON THE USE OF SPEED2 . . . . . . . . . . . . . . . . . . . 17

9.1 Choice of NROW and NCOL . . . . . . . . . . . . . . . 17

9.2 Instrument Definition Cards . . . . . . . . . . . . . . . . 18

9.3 Unconverted Input . . . . . . . . . . . . . . . . . . 18

9.4 Converted Input . . . . . . . . . . . . . . . . . . . . 19

9.5 Creating New Instruments . . . . . . . . . . . . . . . 19

10. DIAGNOSTIC AND ERROR MESSAGES . . . . . . . . . . . . . . . . 20

10.1 Unconverted Data Checking . . . . . . . . . . . . . . . 20

10.2 Data Cards and Format Errors . . . . . . . . . . . . . . 21

10.3 Other Messages and Diagnostics . . . . . . . . . . . . . 22

11. DATA CARDS FOR THE EXECUTION OF SPEED2 . . . . . . . . . . . . . 24

11.1 SPEED2 Data Cards . . . . . . . . . . . • . . . . . . . 24

11.2 PLOTl Data Cards . . . . . . . . . . . . . . . . . 24

11.3 PLOT2 Data Cards . . . . . . . . . . . . . . . . 25

11.4 PLOT3 Data Cards. . . . . . . . . . . . . . . . . 25

11.5 PLOT4 Data Cards. . . . . . . . . . . . . . . . 26

12. REFERENCES . . . . . . . . . . . . . . . . . . . 26

APPENDIX A. PROGRAM STATEMENT LISTING OF SPEED2 . . . . . • • • . • . A-1

APPENDIX B. SAMPLE RUNSTREAMS AND EXAMPLES OF THE USE OF SPEED2 • • B-1 
Table 1. Recording format of several data acquisition systems . . . . . 27 Table 2. Data acquisition system formats . . . . . . . . . . . 28 Table 3. SPEed 2 data cards. . . . . . . . . . . . . . . . . . 37 Table 4. PLOTl data cards . . . . . . . . . . . . . . . . . 38 Table 5. PLOT2 data cards . . . . . . . . . . . . . . . . . . 44 4 Table 6. PLOT3 data cards . . . . . . . . . . . . . . . . . 53

Table 7. PLOT4 data cards . . . . . . . . . . . . . . . . 57 
Richard D. Peacock and John M. Smith

The voluminous amount of data that can be collected by automatic data acquisition systems requires the use of a digital computer for the reduction of data. A general purpose computer program for the reduction of data collected by automatic data acquisition systems is presented. The program is written with the ability to accept data from a number of different data acquisition systems, with the ability to check the correctness of data included. Through the use of FORTRAN computer programming, the data can be converted to meaningful scientific and engineering units. The data can then be presented in tabular, printer plot or ink pen plot form.

The program is documented, and detailed instructions for its use, with examples, are presented. The use of SPEED2 requires some knowledge of FORTRAN programming language and the executive control language for the computer system in use.

Key words: Computer program; data reduction; data acquisition systems; plotting.

\section{INTRODUCTION}

In 1968, the Building Research Division of the National Bureau of Standards (NBS) approached the Computer Services Division of NBS with a proposal concerning the design of a series of computer programs to facilitate the analysis of automatically recorded data. During the following two years, a system of computer programs called SPEED (Systematic Plotting and Evaluation of Enumerated Data) was developed and tested. This system was announced at the Ninth Annual Technical Symposium of the Association for Computing Machinery and in an article in Computer Graphics. The following paragraphs, quoted from the Computer Graphics article, which indicated the need for SPEED are still valid: 
"During the past ten years the use of data acquisition systems or digital scanning systems has increased many fold. Such systems record automatically the readings of a number of instruments on either punched paper tape or magnetic tape. The individual characteristics of the various commercially available digital scanning systems vary greatly; however as a point of reference it may be noted that: one commercially available system is able to record the readings of up to one thousand instruments; another digital scanning system is able to read a clock and record the time plus the readings of up to twenty-five instruments in 2.3 seconds.

The use of digital scanning systems offers several advantages to the research scientist. First, their rapid recording capabilities allow for more complete data sampling. Second, automatically recorded data is more accurate than data that has been recorded manually.

These advantages are however, to some extent, counterbalanced by several problems which arise. Two problems are caused by the large volume of recorded data. First, it is difficult, if not impossible, to process large volumes of data by hand. Thus, the scientist finds it necessary to make use of the computer. Unfortunately, he is often unfamiliar with the capabilities and limitations of this device. Second, when presented with a large volume of data, it is often difficult for the scientist to rapidly interpret the broad characteristics of general trends that may be present. Two other problems arise in the form in which the data are recorded. The data are generally recorded in millivolts rather than standard units. Thus, some conversion process, usually a linear transformation, is required. Furthermore, the recorded data are not usually directly compatible with computers. In order for a computer to read this data some special computer program must be used to read this data in the recorded form and translate it into the internal computer representation."[1] ${ }^{1}$

\footnotetext{
Numbers in brackets refer to the literature references listed at the end of this report.
} 
During the nine years since its announcement, SPEED has been widely used at NBS and other computer installations. SPEED has operated successfully on UNIVAC, IBM, and CDC large-scale computers and has proved its usefulness. Unfortunately, during those nine years, many users found it convenient to modify the original system. This has led to the existence of different versions of SPEED, many of which do not include some important features, and few of which correspond directly to the original (and only) documentation.

For these reasons, SPEED has been rewritten with the new system titled SPEED2. The goals of this task have been:

a. To provide a standardized system of programs with appropriate current documentation;

b. To recover features lost through evolution of SPEED, in particular, the handling of input data;

c. To provide a more easily readable program including all old features and several new features; and

d. To take advantage of changes in computer operating systems.

The remainder of this paper provides a description of SPEED2 and instructions for its use.

Although this paper is meant as a detailed guide to the use of the SPEED2 system, some knowledge of FORTRAN programming and executive system control language for the computer in use is assumed.

\section{SPEED2, PURPOSE AND STRUCTURE}

\subsection{Purpose of SPEED2}

SPEED2 is designed to aid in the analysis of experimental data composed of a set of readings from a series of instruments. Thus, the basic data for SPEED2 are represented as a matrix

$$
\operatorname{REED}(i, j)
$$

where the row index, $i$, refers to a given scan through the series of instruments, with $i=1$ corresponding to the first scan, etc. Typically, 
data acquisition systems scan at a fixed interval of time, so that the row index will also refer to a single time since the start of a test. The column index, $j$, refers to the readings from a single instrument. The order of the instruments in the columns of the array is controlled by the user through data cards read during the execution of the program.

SPEED2 provides the capability to read the input matrix from a variety of media including magnetic tape, paper tape, mass storage devices, or punched cards (of unconverted or converted data). This includes input checking and appropriate error messages. The ability to modify (correct or alter) individual entries, portions of rows or portions of columns, or entire rows or columns of the matrix is included in SPEED2. SPEED2 also provides for the transformation of analog instrument readings into standard units through linear or nonlinear transformations. This transformed and corrected matrix may be outputted to a printer, card punch, magnetic tape, or mass storage device. The user may generate printer plots or ink pen plots of the readings of any instrument versus a series of readings from other instruments. Finally, the ability to compare entries of several matrices, or to merge several matrices, each representing a different experiment, is included.

\subsection{Structure of SPEED2}

The system, SPEED2, is composed of a main program, ambiguously titled SPEED2; four major subprograms named PLOT1, PLOT2, PLOT3, and PLOT4; and a number of minor subprograms. Several of these subprograms are proprietary and/or limited in use to a UNIVAC computer. Consistent use of variable names among the various subprograms has been largely achieved.

The major subprogram, PLOTl, provides a means to read, check, and correct the input data recorded by a variety of data acquisition systems as shown in table 1 . In addition, the user may specify a unique data acquisition system through card input.

The major subprogram, PLOT2, and its dependent subroutines have the role of reading, correcting, transforming, and printing of the input matrix. This segment of the system also generates printer plots.

The system segment controlled by PLOT3 may be used to generate pen plots on a Calcomp plotter. 
The segment controlled by PLOT4 is used to make comparisons between several input matrices of different experiments.

\section{MAIN PROGRAM, SPEED2}

The main program of the SPEED2 system is also titled SPEED2. Although a relatively short program, SPEED2 serves several vital functions.

First, SPEED2 provides dimensioning for many of the arrays used in the system that change in size depending on the size of the input matrix. For the NBS UNIVAC Computer, these dimensions are controlled by PARAMETER cards in the program SPEED2 as shown in appendix $A$ :

\section{PARAMETER NROW $=30$, NCOL $=50$ \\ PARAMETER MAXPLT $=6$ \\ PARAMETER MAXCNL $=150$}

The entries on the first card, NROW and NCOL, refer to the number of rows (scans) and columns (instruments), respectively, in the input matrix. The second parameter card contains a single parameter, MAXPLT, which specifies the maximum number of curves to be plotted on any one graph in PLOT2 or PLOT3. The parameter MAXCNL defines the actual maximum number of instruments recorded by the data acquisition system in a single scan. It may be larger or smaller than the parameter NCOL. A proper choice of values is important to the efficient operation of the system.

Second, SPEED2 provides direction to the system. Through an input card, the user indicates his intention to use a particular major subprogram, PLOT1, PLOT2, PLOT3, or PLOT4. SPEED2 reads this input card and calls the proper subroutine. The user also has the options, as indicated through an input card, to process several data sets in sequence. SPEED2 provides this capability, and also erases all input from one data set before proceeding to the next.

\section{PLOTI, PURPOSE AND STRUCTURE}

PLOTl, the first of the major subprograms of SPEED2, provides for the initial input of data from a data acquisition system; checking of this input data; and optional output of the data to card punch, printer, or mass storage. With a minimum of data cards, the user can check the correctness of the data recorded by a data acquisition system. 
PLOT1, like SPEED2, reads a data card which indicates the user's choice of a number of options. The parameters are interpreted, and the appropriate subroutines are called to perform the desired tasks.

The first card read by PLOTl contains a series of parameters which indicate the particular input media and data acquisition system used to record the data, and the action to be taken with the input data matrix. options include printing all, some, or none of the input records; punching the input data onto punched carảs or writing to mass storage or magnetic tape; printing messages when errors are encountered in the input data; and skipping particular records of data.

In addition to this parameter card, PLOTl requires two cards which indicate the title of the experiment to be printed at the top of each page of output. All other cards are optional, as detailed in section 11.

\section{PLOT2, PURPOSE AND STRUCTURE}

PLOT2 is the second of the major subprograms of SPEED2. In addition to all the features of PLOT1, PLOT2 provides for the conversion of analog instrument readings into standard units through linear or nonlinear transformations and for analysis of the input matrix.

PLOT2, like SPEED2 and PLOT1 reads data cards which indicate the user's choice of a number of options. These cards are interpreted, and proper subroutines are called to perform the desired tasks.

The first card read by PLOT2 contains a series of parameters which indicate the particular input media of the data matrix (magnetic tape, paper tape, unconverted data cards, converted data cards, etc.), the data acquisition system used to record the data, and the action to be taken with the input data matrix. Options include printing all, some, or none of the records; punching the input data on cards or writing to mass storage; stopping or proceeding after reading the input; printing some, all, or no input error messages; and skipping specified data records.

Next, a series of parameters is read which indicate actions to be taken with the converted data matrix. Options include printing, punching, and writing the converted matrix; generating graphs; making corrections to the matrix; and stopping after the conversion process is completed. 
The remainder of the input is composed of the experiment title, cards defining the instruments (or columns), corrections (if any), and directions for the generation of graphs.

\subsection{Using PLOT2}

In order to use PLOT2 efficiently, the user should first examine the data collected by the data acquisition system to determine the number of rows and columns needed to store the input matrix. The user should also determine at this point whether or not new columns, which augment the input matrix, are to be generated through the use of subprograms CONV and FIND. If necessary, cards should be prepared to change the parameters which define the storage area for the input matrix and cards should be prepared to modify subprogram CONV. Instructions for changing subprogram CONV are given in section 5.2, and examples of both types of changes described above are given in appendix $B$. These cards should be preceded by the required executive system cards and followed by the appropriate executive system cards necessary to create and run an executable program.

The user then prepares the data cards. The format and content of each data card is given in section 11. It should be noted that the main program, SPEED2, requires two data cards prior to those cards required by PLOT2.

\subsection{Data Transformation}

As previously noted, instrumentation data readings are normally recorded in millivolts or volts. To convert these readings to standard units of temperature, length, etc., it is often sufficient to transform the reading through a linear equation. Some conversions, however, are considerably more complex and nonlinear.

\subsubsection{Linear Transformations}

PLOT2 achieves linear transformations as follows: The input data to PLOT2 includes a series of cards which describe each instrument. For each instrument, these cards contain the instrument's number, the instrument's type, and the instrument's name. The name is used only for identification purposes in printing results. The instrument's number is the channel number 
assigned by the data recording device. The instrument type is a user assigned number. Generally, instruments requiring the same linear transformation are assigned the same type. However, note that there may be only one instrument of type 1 (which usually records time). Following the instrument descriptors, PLOT2 requires a series of coefficient cards. These cards contain the transformation coefficients for each type used, I through $n$ (assuming $n$ different instrument types have been assigned).

All of this data is passed from PLOT2 to subprogram CONV. For each instrument, readings have been stored in the columns of the input matrix REED. Thus if instrument number $j$ appears on the $i$ 'th descriptor card, its readings are

$$
\operatorname{REED}(\ell, i), \ell=1, \operatorname{MAXR}(i)
$$

where MAXR(i) is the number of readings of instrument number $j$. Furthermore, if the type of instrument number $j$ is $k$, that is ITYPE (i)=k, then the appropriate transformation coefficients are $C(k)$ and $\operatorname{ADD}(k)$. Hence, the transformation required is

$$
\operatorname{REED}(l, i)=\operatorname{REED}(l, i) * C(k)+\operatorname{ADD}(k), \ell=1, \operatorname{MAXR}(i)
$$

\subsubsection{More Complex Transformations}

Nonlinear or more complicated transformations require the user to make modifications to subprogram CONV. Such modifications are made by computer programming using ordinary FORTRAN statements. Clearly, the user will wish to modify a given column of the input matrix; however, the user may not know which column of the matrix contains the readings of the instrument in question. This may be answered through the use of subprogram FIND.

Subprogram FIND has four arguments and is invoked through a FORTRAN statement of the form

$$
\text { CALL FIND (J, I, KH, NCOL) }
$$

where $\mathrm{KH}$ is the array of instrument numbers in use, NCOL is the maximum dimension of that array, and $J$ is an instrument number. The value of $I$, is computed by FIND and, is the column of the matrix in which the readings 
of instrument $\mathrm{J}$ have been stored. A convenient convention to follow

is the form

CALL FIND $(72, \mathrm{~L} 72, \mathrm{KH}, \mathrm{NCOL})$

In this example, the readings of instrument number 72 will be found in column $\mathrm{L} 72$ (location of instrument 72 ).

Having located the proper columns of the matrix, nonlinear transformations are carried out through FORTRAN arithmetic statements. Thus the following simple FORTRAN DO loop

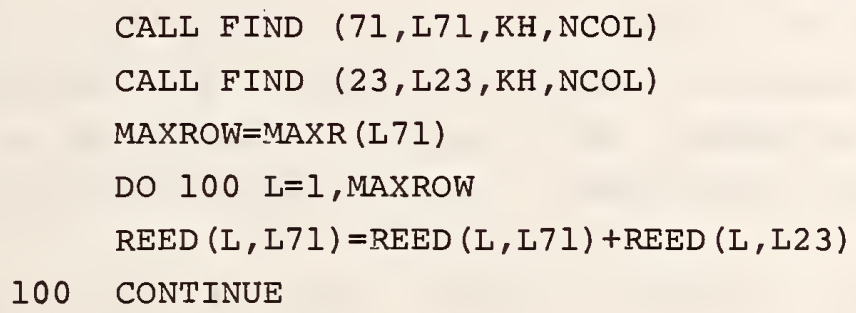

would transform the readings of instrument number 71 by adding to it the readings of instrument number 23 . A series of nonlinear transformations might be programmed as shown below:

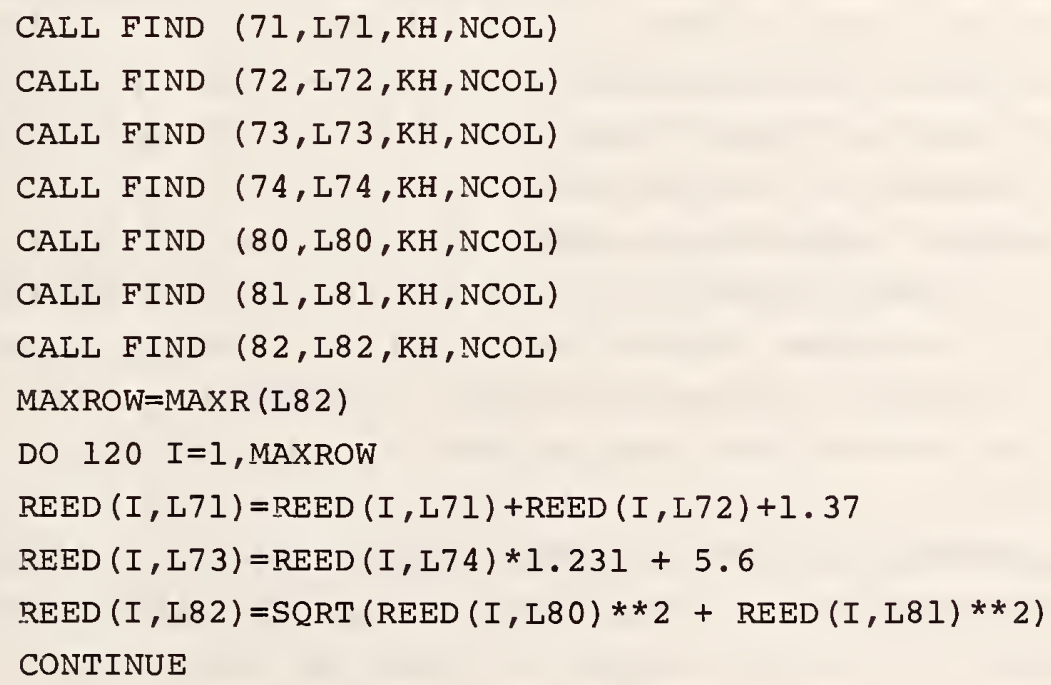

The first seven cards call subprogram FIND as described above to locate the instruments to be converted. The instruments are then used in simple calculations to serve as an example of the use of subprogram CONV. Obviously, much more complex conversions could be carried out. 
The above cards should be inserted in subprogram conv' as noted in the examples shown in appendix B.

\subsection{Making Corrections to the Data Matrix}

The PLOT2 segment also provides the capability of modifying or correcting entries in the input matrix. Any number of corrections may be made; however, any single correction may apply only to one single entry, consecutive entries in a single column, or consecutive entries in a single row. Corrections are expected if and only if the PLOT2 parameter NCORR is set non-zero by the user.

Assuming NCORR is non-zero, PLOT2 will read a card containing the variables IRL (a low row index), IRH (a high row index), ICL (a low column index), and $\mathrm{ICH}$ (a high column index). The following restrictions apply:

a. Either IRL=IRH or ICL=ICH or both. Note, if IRL=IRH, all corrections apply to a single row. If ICL=ICH, all corrections apply to a single column. If IRL=IRH and ICL=ICH, a single entry will be corrected.

b. IRL is greater than zero but less than or equal to IRH or IRI is less than zero. IRI less than zero signifies the end of the corrections.

c. ICL is greater than zero but less than or equal to ICH, or both ICL and ICH are less than zero. Note that both ICL and ICH must be both positive or both negative. If they are negative, they are interpreted to be instrument numbers rather than column numbers. In this case, the colunn $i$ in which instrument number ICL is stored is found. Similarly, the column $j$ in which instrument number ICH is stored is found. Then the values of ICL and ICH are replaced by $i$ and $j$ respectively. The restriction becomes $i$ is greater than zero but less than or equal to $j$.

Failure to satisfy any of the above restrictions will result in an error message being printed, and may result in all following data cards being out of order. Thus, particular care must be taken in the preparation of this card.

There are, in effect, only three valid combinations. They are:

a. IRL is equal to IRH and ICL is equal to ICH meaning correct entry REED (IRL, ICL) . 
b. IRL is less than IRH and ICL is equal to ICH meaning correct entries REED(IRL, ICL) REED (IRL+l,ICL), ..., REED (IRH-1, ICL), and REED (IRH, ICL) .

c. IRL is equal to IRH and ICL is less than ICH meaning correct entries REED (IRL, ICL), REED (IRL, ICL +1 ), ..., REED (IRL, ICH-1), and REED (IRL, ICH) .

In any of the above cases, the number of entries to be corrected is

$$
(I R H-I R L)+(I C H-I C L)+I
$$

These entry corrections are read from a series of data cards punched in the format $8 \mathrm{~F} 10.0$.

\subsection{Generating Graphs}

PLOT2 provides the capability to generate printer plots of the readings of either one or more instruments versus those of several other instruments. This is done only if the input parameter NPLOT is set non-zero by the user.

The program PLOT2 reads a card, containing up to 80 characters, which is printed as a heading on the graph. The program also reads a card or cards which contains the word PLOT (in any four consecutive columns, followed by a series of instrument numbers that specify the instruments to be plotted typed in free format separated by at least one space. There are two allowable formats for plot cards. If the card contains a comma separating instruments into pairs such as

$$
\text { PLOT } \quad 3999,3017 \quad 5999,5017 \quad 3999,10017
$$

then the readings from the first instrument of each pair are taken to be $x$-values (abscissas) and the second instrument of each pair are taken to be $y$-values (ordinates). If no comma is found on any of the cards, such as

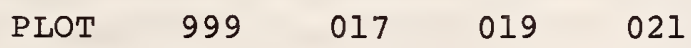

then, the first mentioned instrument's readings are taken to be $x$-values (abscissas), and readings from the other entered instruments are taken to be $y$-values (ordinates). From one to $n$ curves may be plotted simultaneously on a single graph, where $n$ equals the SPEED2 parameter MAXPLT. 
The user also controls the lower and upper limits of the two axes. $A$ card is read containing the desired lower and upper limits of the $x$ and $\mathrm{Y}$ axes, $\mathrm{XL}, \mathrm{XH}, \mathrm{YL}$, and $\mathrm{YH}$, respectively. Care should be taken in choosing the limits to insure that readable values are printed on the axes. One important note is that points which fall outside the ranges determined for the two axes, are neither plotted nor noted.

Finally, the program reads a single card with two messages of 40 characters each. The first is printed vertically as a title for the $y$-axis, and the second is printed below the graph as a title for the $\mathrm{x}$-axis.

After printing the graph, the program seeks another set of graph defining cards. A blank card signals the end of the plotting phase which is the final portion of PLOT2.

\section{PLOT3, CALCOMP PEN PLOTTING OF DATA}

The third of the major subprograms of SPEED2, PLOT3, allows the user to prepare high quality ink pen plots. PLOT3 is designed to accept data generated by PLOT2 and prepare a magnetic tape to be plotted off-line on a Calcomp plotter. Many of the subroutines used by PLOT3 are proprietary, however, and may be used only by license from California Computer Products, Inc. Like the other major subprograms, PLOT3 reads data cards which indicate the user's choice of a number of options to control the generation of the plots.

The first two cards read in the PLOT3 phase contain parameters which specify certain parameters which control the generation of the graphs produced. The user can specify the type of curve generated, either a smooth, fitted curve, or a curve connecting points on the graph with straight lines; whether to print or not to print the data; the course of action to be taken if points are found that would be out of bounds on the graphs produced; whether to put axes on two or four sides of the plots; the spacing of plotting symbols on the curves produced; and whether or not to read cards to produce legends on the curves. In addition, the user can specify the size of the horizontal and vertical axes of the graphs, and a scaling factor to increase or decrease the overall size of the plots produced. 
A number of cards are then read which specify the experiment title, define the instruments, and specify the plots to be produced, in a fashion similar to that used in PLOT2. Note that no conversion of data is made in PLOT3. Only reduced data (data processed by PLOT2) is read by PLOT3.

\subsection{Using PLOT3}

As with PLOT2, some forethought should be given to the data to allow the most efficient use of the SPEED2 system. The user should examine the data to determine the number of rows and columns needed to store the input matrix and the maximum number of curves on any single graph. If necessary, cards should be prepared to change the parameters which define the storage area for the input matrix exactly as was done with PLOT2. Of course, these cards should be preceded by and followed by the necessary executive system control cards to create an executable program.

The user then prepares the data cards. The format and content of each card is defined in section 11. As with PLOT2, SPEED2 requires data cards prior to those required by PLOT3.

\subsection{Generating Pen Plots}

PLOT3 is designed to prepare a magnetic tape to be plotted on a calcomp plotter. For each plot prepared, the readings of one or more instruments are plotted versus those of one or more other instruments.

A card is read which contains a figure number and two 39 character title lines which are placed below the plot as the title of the plot. Also read is a card containing two messages of 40 characters each. The first is printed as the title for the $y$-axis, and the second for the x-axis. All of the titles are centered along their respective axes.

PLOT3 has the capability to produce plots with linear, semi-log (with the $\log$ axis along the $\mathrm{y}$-axis), or $\log \log$ axes. The program reads a card or cards containing either the word PLOT for a linear plot, SPLOT for a semi-log plot, or LPLOT for a log-log plot in any consecutive columns followed by a series of instrument numbers that specify the instruments to be plotted. As with the PLOT2 printer graphs, there are two allowable 
formats for the cards. If the card contains a comma separating instruments into pairs, such as

$\begin{array}{rlll}\text { PLOT } & 3999,3017 & 5999,5017 & 3999,10017 \\ \text { SPLOT } & 3999,3017 & 5999,5017 & 3999,10017 \\ \text { LPLOT } & 3999,3017 & 5999,5017 & 3999,10017\end{array}$

the readings from the first instrument of each pair are taken to be $x-$ values (abscissas), and the second instrument of each pair is taken to be $y$-values (ordinates). In the above statements, instrument 3999 is plotted versus 3017,5999 versus 5017, and 3999 versus 10017 . If no comma is found on any of the cards, such as

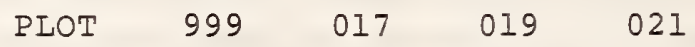

then the first mentioned instrument's readings are taken to be $x$-values (abscissas) and the readings from the other entered instruments are taken to be $y$-values (ordinates). As with PLOT2, up to MAXPIT curves may be plotted on a single graph. If a card containing instrument numbers contains a semicolon in column 80 , then another card is read and interpreted as containing additional instrument numbers for plotting on the same graph. As many cards as are needed may be used to specify instrument numbers.

The user can also control the lower and upper limits of the two axes. A card is read containing the lower and upper limits of each axis. Care should be taken in choosing the limits to insure that readable values are printed on the axes. However, PLOT3 checks these numbers to insure that readable numbers will be printed and may modify the limits to insure readability.

Finally, the program reads a single card containing two messages of 40 characters each. The first is printed as the title for the $y$-axis, and the second for the x-axis. As with the graph heading, the axis titles should be centered in their 40-character spaces to insure that they will be centered properly on the plot.

For example, the following sequence of cards

$\begin{array}{llll}\text { SPLOT } & 999,017 & 36,123 & 231,84 \\ & 93,998 & & 41,86 \\ & 78,81 & & \\ 0.0 & 250 . & 0.001 & 100 .\end{array}$


OIRATE OF DECOMPOSITION

TIME

(HOURS)

RATE

(1/SEC)

would prepare a single semi-log graph of instrument 999 versus 017,36 versus 123, 231 versus 84,93 versus 998,41 versus 86 , and 78 versus 81 . The $x$-axis limits would be from 0.0 to 250. and the y-axis (a log scale) from 0.001 to 100. The title for the plot would be FIGURE 1: RATE OF DECOMPOSITION. The $x$ and $y$ axes would be labeled TIME (HOURS) and RATE (1/SEC), respectively.

PLOT3 also provides a means for the user to place a legend on the graph. The legend can be positioned anywhere on the graph and lines of text, up to 60 characters each, can be optionally prefixed with a symbol to identify legends for individual curves.

After generating the necessary information on the magnetic tape, the program seeks another set of graph defining cards. A blank card signals the end of the plotting, and the program prepares the tape for plotting.

\section{PLOT4, COMBINING SEVERAL SETS OF DATA}

PLOT4 is the last of the major subprograms of the SPEED2 system. PLOT4 allows the user to perform analysis and plotting on a number of instruments from several different tests, possibly with repeated instrument numbers in the tests. By renumbering the instruments to include a test number for each instrument, PLOT4 creates a new data set consisting of the readings of the renumbered instruments. Further analysis may then be performed on this new data set using PLOT2 or PLOT3 as desired.

As an example, consider an instrument numbered 121 from 11 different tests numbered arbitrarily test 3 through test 13. PLOT4 would perform a renumbering of the 11 instruments as $3121,4121,5121,6121,7121,8121$, 9121, 10121, 11121, 12121, and 13121, respectively. These instruments may then be punched on cards or written to mass storage and referenced in either PLOT2 or PLOT3 as reduced data using the new instrument numbers 3121 through 13121.

\subsection{Using PLOT4}

As with the previous major phases of SPEED2, the user should determine the number of rows and columns needed to store the readings of the instruments 
to be processed. If necessary, cards should be prepared to change the parameters which define the storage area for the input matrix. of course, these cards should be preceded by the required executive system cards and followed by the appropriate executive system cards necessary to create an executable program.

The user then prepares the data cards. The format and content of each data card is described in detail in section 11 and generally outlined below. It must be noted that as with PLOT1, PLOT2, and PLOT3, the main program, SPEED2 requires two data cards prior to those required by PLOT4.

\subsection{Renumbering of Instruments Using PLOT4}

The first card read by PLOT 4 contains two parameters that specify whether the data is to be printed and/or punched onto cards or mass storage. After reading title cards, cards defining the instruments to be selected from the various tests are included. Each instrument card contains a test number, provided by the user; an instrument number within the test; and a short description of the instrument. The remainder of the cards is comprised of the reduced data from each test preceded by a card of the form

TEST nnn

defining a test number for each test. Specifications for the data cards are given in section 11 along with examples of the use of PLOT4 in appendix B.

\section{RESTRICTIONS ON THE USE OF SPEED2}

The system, SPEED2, has been tested and continues to be tested by the authors. Users are requested to report any problems which arise through the use of SPEED2. Users should not modify any of the programs in this system with the exception of changing the parameters (see section 3 ) and inserting nonlinear transformation statements in subprogram CONV (see section 5). Examples of these changes are given in appendix B. Any other substantive changes are strongly discouraged without a detailed knowledge of the internal structure of the SPEED2 system. 
While most of SPEED2 is written in a portable subset of FORTRAN IV and thus is compatible with a variety of computers, a number of exceptions were found when the subprograms were verified using the VERIFIER developed by Bell Laboratories [2]. These are described below:

a. Subprograms EREXIT, BYTE and NTRAN are written in UNIVAC assembler language. These would have to be replaced with the equivalent routines to be used on another machine.

b. A number of subprograms make use of Hollerith literals in arithmetic operations. To use SPEED2 on a computer other than a UNIVAC 1100 series computer, the computer must have the ability to arithmetically manipulate character strings.

c. The PARAMETER statement used in the main program, SPEED2, is unique to UNIVAC FORTRAN. Actual dimension limits for the variables NROW, NCOL, MAXPLT, MAXCNL, INDIM, OUTDIM, NPTS, and PLTDIM must be substituted in the main program to use SPEED2 on another computer. An alternate main program that has been successfully used on an INTERDATA 7/32 minicomputer is included in the program listing in appendix $A$.

d. Finally, PLOT3 makes use of a number of Calcomp proprietary subprograms which must be obtained by a given computer installation from California Computer Products, Inc. The authors believe that a detailed description of the many secondary subroutines would be either too brief to be of value or too complex to be comprehended. Thus, such statements have been omitted unless absolutely necessary.

\section{NOTES ON THE USE OF SPEED2}

\subsection{Choice of NROW and NCOL}

Choosing NROW and NCOL should be done with care. If values are chosen which are too small, execution will be terminated when the input matrix size exceeds the allocated size. Conversely, computer use charges are based, in part, on the allocated storage. Too large values of NROW and NCOL will cause excess costs. In addition to the input matrix, REED, being dimensioned NROW by NCOL, the arrays KH, ITYPE, $C, X . Y$, and NAME all depend on NCOL for their size. Thus, the storage allocation for all of these arrays is approximately 
$(N R O W+15) *(N C O L+13)$

On the NBS UNIVAC 1108, this product should not exceed 45000 .

\subsection{Instrument Definition Cards}

As noted in section 5, each instrument is defined by a card containing the instrument number, type, and name. The use of these data in the conversion process has already been explained, but other points should be mentioned.

First, subprogram PRINT prints only those instruments defined through an instrument card. Thus, each instrument whose readings are to be printed must be defined, whether the instrument is real or whether it represents some combination of other instruments formed through the use of subprogram CoNv. Conversely, if the user wishes to divide his experiment into several smaller sub-experiments, he may omit the processing of data for any instruments by omitting their defining cards. One exception to this rule is the instrument card which defines time. If its defining card is left out, the program will internally reserve space for it in the array REED. However, if its card is not included, the time readings will not be available for plotting or conversion.

Second, subprogram PRINT will print the converted instrument readings, in columns, in the order in which the defining cards appear. Thus, the user may predetermine this printout order in whatever way is most convenient and may easily change the order if the computer run is repeated. As previously mentioned, each column will be headed by the first six characters of the instrument's name.

Finally, if the user indicates a desire to punch the converted matrix on cards or mass storage through setting NPNCH non-zero, the above comments apply to these cards too.

\subsection{Unconverted Input}

Data acquisition systems record readings from instruments in a variety of different formats. Table 1 presents the formats used in a number of different data systems. Several characteristics common to the different systems are detailed below. 
a. A time reading is usually prefixed to the series of instrument readings. Time readings and instrument readings are of different formats.

b. Time readings consist of digits to indicate days, hours, minutes, and/or seconds, perhaps separated by spaces or other special characters.

c. Each instrument reading consists of characters to indicate a channel number assigned by the data acquisition system, the sign of the reading, the value of the reading, and a channel overflow indicator.

d. Within a single scan, the time reading and each instrument reading is separated by spaces and/or other special characters.

e. Scans are separated by a special character sequence on card images or by a record separator on magnetic tape.

f. The end of the test data recording is indicated by a special character sequence or by an end of file mark on magnetic tape.

Table 2 presents sample scans from a number of different data systems. Tables 1 and 2 also provide guidelines to follow if cards are to be prepared by the user.

\subsection{Converted Input}

Converted instrument readings also require that data be prepared following a particular format. The readings from each instrument are prepared in a format similar to table 2. Each set of readings from an instrument begins with a data card specifying the number of readings of the instrument and the instrument number in a format $2 I 6$, followed by a series of cards containing the readings. The readings, in the format E10.5, are packed eight readings per card. Finally, the end of test is indicated by a card with 999 in columns 78, 79, and 80 .

\subsection{Creating New Instruments}

Complex transformations made through the use of subpro',ram CONV may include new instruments representing combinations of oth: instruments. 
It should be noted that the user must specify the number of readings in the created instrument by setting MAXR for the created instrument.

\section{DIAGNOSTIC AND ERROR MESSAGES}

The SPEED2 system is designed to intercept and interpret most user errors and to print a diagnostic or error message indicating the error. While most messages are self-explanatory, they are listed below, along with explanations of the errors and how to correct them.

\subsection{Unconverted Data Checking}

When the unconverted data are input to the program in any of the available forms, the data are checked for errors in the format of the data or for illegal characters, etc. If errors are found, a message is printed. Five different messages are possible. The first indicates an error in the unconverted data. This can either be an illegal character in the data, or data in which characters are out of sequence, bad, or missing. The message takes the form

\section{CHARACTER SEQUENCE ERROR RECORD: i CHARACTER: j}

The record index, $i$, is the record (scan) number of the particular record in error and the character index, $j$, is the number of the actual character in error. The normal action taken by SPEED2 is to set the particular reading in error to zero and continue processing. If the user has prepared punched data cards or card images on mass storage of the unconverted data, and the user wishes to correct the error, the data can be edited, corrected, and the program re-run with the corrected set of data. The user could also simply replace the reading in error by using subprogram CORR to correct the reading.

The second type of error message indicates an overflow in a data reading from the data acquisition system. It takes the form

\section{OVERFLOW WARNING RECORD: i CHARACTER: j CHANNEL: $k$}

All comments made for the previous error message also apply for this diagnostic warning. The channel number as recorded by the data acquisition system, k, is also given. 
The third type of message indicates simply that the record length of a single scan through the instruments has changed from the last scan. It is simply a warning to the user that something may be wrong with the data. It takes the form:

RECORD LENGTH WARNING RECORD: i OLD LENGTH: j NEW LENGTH: $k$

The record number, $i$, the length of the last scan, $j$, and the length of the current scan, $k$, is given.

The last two error messages indicate a duplicate time or channel reading in a single scan. The messages take the form:

MULTIPLE TIME ERROR RECORD: i CHARACTER: j

MULTIPLE READING WARNING RECORD: i CHARACTER: j CHANNEL: $k$

The record number, $i$, and the character number, $j$, are given for both messages. The channel number, $k$, is also given for the channel warning.

\subsection{Data Cards and Format Errors}

Many user errors result from improper placement of or from incorrect formats on data cards entered by the user. While it is urged that the user carefully check the data cards before running SPEED2, such errors are bound to occur. Each data card read by SPEED2 has a unique identification (SPEED2 Data Card Al, PLOT3 Data Card D3 for instance). If format errors are found on data cards, program execution is halted and an appropriate error message is printed. Two messages are possible for read errors, but both take the same form:

FORMAT ERROR ON iseg DATA CARD icrd

or

END OF FILE ENCOUNTERED ON iseg DATA CARD icrd PRECEDING DATA CARDS MAY BE IN ERROR

The two variables, iseg and icrd, are the program segment (SPEED2, PLOT1, PLOT2, PLOT3, or PLOT4) and the card identification (A1, D3, etc.). 
Many other error messagts are printed by the SPEED2 system to alert the user of possible errors encountered during the processing of data by SPEED2. A short explanation is provided below for each along with an explanation of how it can be corrected.

\section{ONLY ONE INSTRUMENT OF TYPE 1 IS ALLOWED SECOND FOUND AT INSTRUMENT NUMBER i}

Instrument Type 1 is reserved to indicate the time channel. obviously, only one channel is allowed for this. If more than one is found, program execution is stopped. The user must correct the instrument cards so there is only one instrument of Type 1, the time channel.

\section{COLUMN STORAGE LIMITS EXCEEDED AT COLUMN i CHECK PARAMETERS NCOL AND NROW}

The user has not allowed enough room for the instruments he wishes to process as defined by the instrument defining cards. The PARAMETER NCOI should be increased to the proper size.

\section{ILLEGAL INPUT FILE TYPE SPECIFIED FOR INTYPE}

The user has specified an illegal number for the input file parameter INTYPE, or there is a format error on the data card on which INTYPE is read.

\section{ROW STORAGE LIMITS EXCEEDED AT ROW i}

CHECK PARAMETERS NCOL AND NROW

The user has not allowed enough room for the number of scans in the experiment. The PARAMETER NROW should be increased to the proper size.

READING OF INPUT FILE TERMINATED BY NTRAN ERROR, STATUS: i

An unrecoverable error has occurred while reading the magnetic tape with NTRAN. All data read in and processed up to the point of error is processed however. There are ways to read magnetic tapes with such errors; however, none appear to be very reliable. (UNIVAC 1100 only) 


\section{INPUT RECORD SIZE OF i EXCEEDS BUFFER SIZE CHECK PARAMETER NAXCNL}

The standard version of SPEED2 is configured for a maximum of 150 channels of data per record (scan). More than the maximum number of channels, MAXCNL, have been processed in one record. To correct this, check the input data and/or increase the PARAMETER MAXCNL.

\section{READING TERMINATED BY READ ERROR OR END OF FILE}

Reading of the unconverted input data has been terminated by a read error or by an attempt to read past an end of file. Usually, there is a format error on the input punched cards. The user should check the data to insure it is in the proper format.

NUMBER OF READINGS FOR INSTRUMENT NUMBER i EXCEEDS STORAGE LIMITS. CHECK PARAMETERS NROW AND NCOL

There are too many readings of reduced data for instrument number $i$, or there is a format error on the card defining the instrument in the reduced data set. The user should check the data for correctness and/or increase PARAMETER NROW.

\section{ERROR ON FOLLOWING CORRECTION CARD}

The user has specified NCORR non-zero and subprogram CORR has found an error on the correction cards. The user should check the format of the data cards. The card in error is printed.

\section{ERROR IN SUBPROGRAM FIND}

NO REFERENCE TO INSTRUMENT NUMBER i CAN BE FOUND

Instrument number $i$ was not defined on an instrument card, and the instrument was referenced during transformations using subprogram CONV. The results of the calculations are unpredictable, and the user should check the FORTRAN modifications to subprogram CONV carefully. 
The data cards required for the execution of SPEED2 fall naturally into five major divisions: those cards required by the main program, SPEED2, and cards directing the execution of each of the major phases of SPEED2 -- PLOT1, PLOT2, PLOT3, and PLOT4. In addition, the data cards for each major phase fall into distinct parts. These are described below.

\subsection{SPEED2 Data Cards}

The main program segment, SPEED2, requires two data cards to control the execution of the other program segments. The content and format of these cards is described in table 3. One data card that specifies the number of sets of data is followed by sets of cards defining the major phase of SPEED2 to be used to process each data set and the cards required by the major phase specified on that card. NSETS sets of these cards are included in the total set of data cards.

\subsection{PLOT1 Data Cards}

If the user specified PLOT1 on SPEED2 data card A2, then cards are read as defined in table 4 to control the execution of PLOTl. The six distinct parts defined for PLOTl are:

$\begin{array}{ll}\text { A: PLOTl Control Parameters } & \text { - required } \\ \text { B: Experimental Title } & \text { - required } \\ \text { C: Data Acquisition System Definition } & \text { - optional } \\ \text { (only if INTYPE=0) } & \text { - optional } \\ \text { D: Skipping Records of Data } & \\ \text { (only if INSKIP is non-zero) } & \text { - optional } \\ \text { E: Unconverted Data Cards } & \\ \text { (only if INTYPE specifies cards) } & \text { - optional } \\ \text { F: Converted Data Cards } & \\ \quad \text { (only if INTYPE }=4 \text { ) }\end{array}$

Data cards Part $C$ through $F$ are optional and are only required depending upon the values of certain control parameters defined in Part $\mathrm{A}$. 
The data cards required for the execution of PLOT2 fall naturally into nine distinct parts. Not all parts are required in each run. Whether or not a certain part is required depends on the values of certain input parameters described below and detailed in table 5 .
A: PLOT2 Control Parameters
- required
B: Experiment Title and Instrument Description - required
C: Data Acquisition System Definition
- optional
(only if INTYPE=0)
D: Skipping Records of Data
(only if INSKIP is non-zero)
E: Unconverted Data Cards
- optional
(only if INTYPE specifies cards)
F : Converted Data Cards
- optional
(only if INTYPE=4)
G: Reading Corrections to Data
- optional
(only if NCORR is non-zero)
$\mathrm{H}$ : Cards Read By the User in CONV
- optional
I: Generating Printer Plots
- optional
(only if NPLOT is non-zero)

\subsection{PLOT3 Data Cards}

The data cards for the execution of PLOT3, described in table 6 , fall into four distinct parts. All parts are required for execution.
A: PLOT3 Control Parameters
- required
B: Experiment Title and Instrument Definition
- required
C: Converted Data Cards
- required
D: Generating Pen Plots
- required 
The data cards required for the execution of PLOT4 are described in table 7. The three major parts are:
A: PLOT4 Control Parameters
- required
B: Experiment Title and Instrument Definition - required
C: Converted Data Cards for Each Test
- required

\section{REFERENCES}

[1] Smith, John M., Automatic data evaluation, manipulation, display, and plotting with SPEED, Computer Graphics, Vol. 4, No. 2, 41-53 (Fall 1970).

[2] Ryder, B. G., The FORTRAN verifier: user's guide, Bell Telephone Laboratories, Incorporated, Murray Hill, New Jersey. 


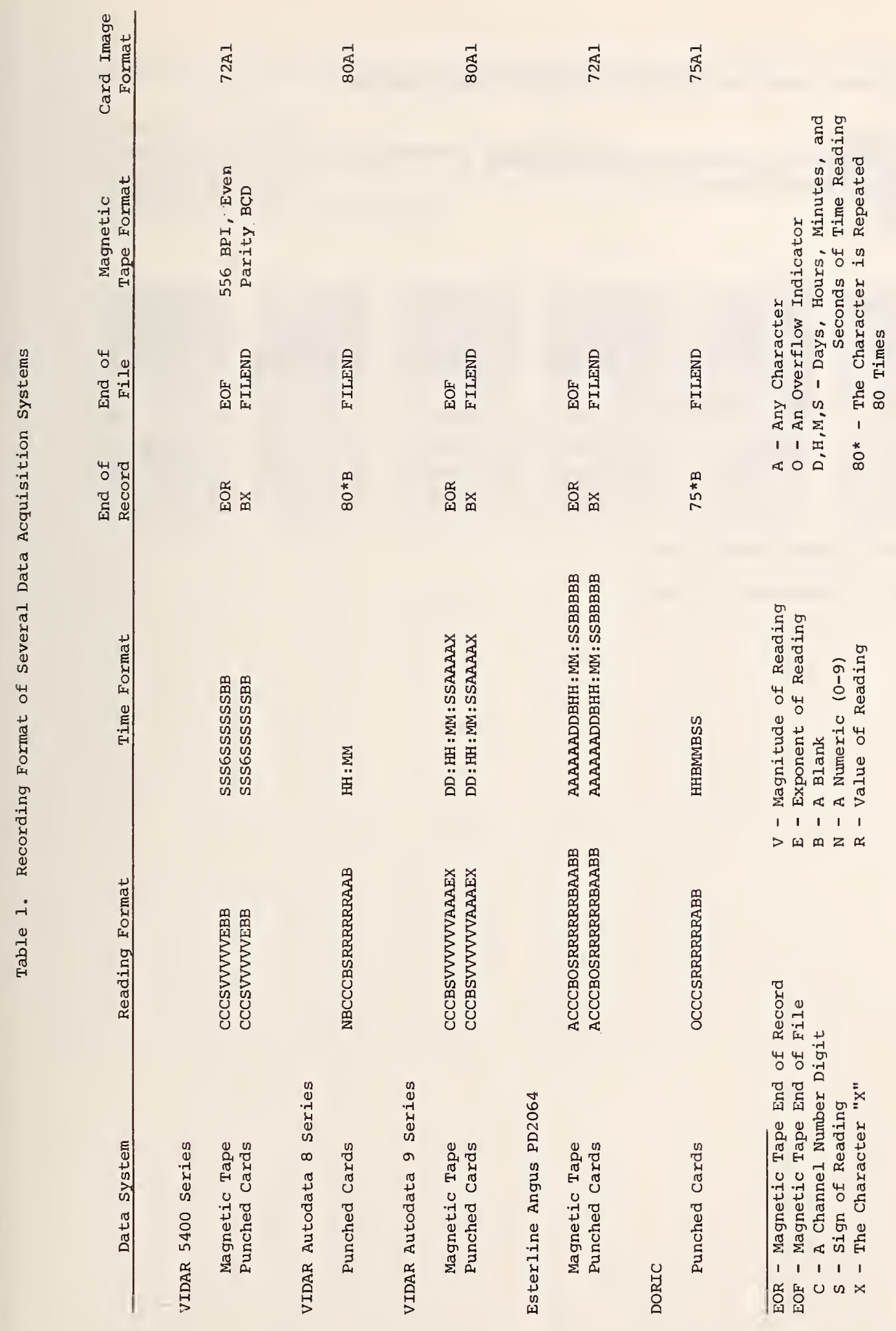


SPEED2 Data Acquisition System Format Specification

INPUT $=$ TAPE

READING $=3 *(C)(+1++-2--0900) 5 *(V)(E) 2 *(K)$

TIME $=3 *(S)(K G) 6 *(S) 2 *(K)$

$E O R=E O R$

$E O F=E O F$

0006000000

0051000526

0112000016

0172000015
0001000166 0061000036 0121000026 0181000026
0012000016 0072000016 0132000016 0191000066
0021000036 0081000016 0141000036 0201000036
0031000015 0092000016 0151000036 0212000006
0041000015 0101000046 0162000016 0221000026

(MAGNETIC TAPE RECORD GAP)

$000 \in 000003$

0051000026

$011200001 \epsilon$

0172000016

0002000006 0061000016 0121000036

0181000036

0011000016 0072000016 0132000016 0192000006
0021600036 0082000006 0141000026 0201000046
0031000026 0092000006 0151000046 0212000016
$0 C 42000016$ 0101000046 0162000016 0222000016

(MAGNETIC TAPE RECOFD GAP)

(MAGNETIC TAPE END OF FILE) 
Table 2. Data Acquisition System Formats. cont. VIDAR 5400 SERIES

SPEED2 Data Acquisition System Format Specification

INPUT $=$ CARD I MAGES

RE ADING $=3 *(C)(+1++-2--0900) 5 *(V)(E) 2 *(K)$

TIME $=3 *(S)(K 6) 6 *(S) 2 *(K)$

$E C R=\{K\}(K X)$

$E O F=(K F)(K I)(K \perp)(K E)(K N)(K D)$

000600.0000 0051000026 0112000016 0172000016 0006000003 0051000026 $011200001 \epsilon$ $01720 C .0016$ FILEND
0001000166 0061000036 0121000026 0181000026 0002000006 0061000016 0121000036 0181000036
0012000016 0072000016 0132000016 0191000066 0011000016 0072000016 0132000016 0192006006
0021000036 0081000016 0141000036 0201000036 0021600036 0082000006 0141600026 c 201000046
0031000016 0092000016 0151000036 0212000006 0031000026 0092000006 0151000046 0212000016
0041000016 0101000046 0162000016 $x$ 0042000016 0101000046 0162000016 $x$ 
Table 2. Data Acquisition System Formats, conto SPEED2 REDUCED DATA

SPEED2 Data Acquisition System Format Specification

$3573150 T C \quad 1$

$.27000+02 \cdot 27000+02 \cdot 27000+02 \cdot 27000+02 \cdot 270 c 0+02 \cdot 27000+02 \cdot 27000+02$ $.27000+02 \cdot 27000+02 \cdot 27000+02.27000+02.27000+02.27000+02 \cdot 27000+02$

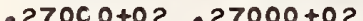
$.27000+02 \cdot 27000+02$ $.27000+02$ $.27000+02$ $000+027000+02$ $.27000+02 \cdot 27000+02 \cdot 27000+02$ $.27000+02.27000+02 \cdot 27000+02$ $27000+02 \cdot 27000+02 \cdot 27000+02 \cdot 27000+02$ $.27000+02 \cdot 27000+02 \cdot 27000+02 \cdot 27000+02$ $.27000+02 \cdot 27000+02 \cdot 027000+02 \cdot 2$ $.27000+02 \cdot 27000+02 \cdot 27000+02$ $.27000+02 \cdot 27000+02.27000+02$ $.27000+02 \cdot 27000+02 \cdot 27000+02$ $.27000+02 \cdot 27000+02 \cdot 27000+02$ $.27000+02 \cdot 27000+02 \cdot 27000+02$ $.27000+02 \cdot 27000+02 \cdot 27000+02.2$ $.27000+02.27000+02.27000+02$. $.28000+02 \cdot 27000+02 \cdot 28000+02$ $.28000+02 \cdot 28000+02 \cdot 27000+02$. $.28000+02 \cdot 28000+02 \cdot 28000+02$ $.28000+02 \cdot 27000+02 \cdot 27000+02$. $.28000+02.28000+02.28000+02$ $.28000+02 \cdot 28000+02.28000+02$ $.28000+02 \cdot 28000+02.28000+02$ $.28000+02 \cdot 28000+02 \cdot 28000+02$ - 28000+02 .28000+02.28000+02 . 28c00+02 . $28000+02 \cdot 28000+02$ $.2800 j+02 \cdot 28000+02.28000+02$ . $29000+02 \cdot 25000+02 \cdot 29000+02$ - $29000+02 \cdot 29000+02 \cdot 29000+02$ $.290 \mathrm{C} 0+02 \cdot 29000+02 \cdot 29000+02$ . 25000+02.29000+02.25000+02 $.30000+02$ . $300 \mathrm{C} 2+02$ $.30000+02$ $.30000+02$ $.30000+02$ $.31000+\mathrm{C} 2$ $.31000+02$ . $31000+02$
$27000+02 \cdot 27000+02$ $.27000+02 \cdot 27000+02$ $.27000+02 \cdot 27000+02$ $.27000+02 \cdot 27000+02$ $.27000+02 \cdot 27000+02$. $27000+02 \cdot 27000+02$ $.27000+02 \cdot 27000+02$ $27000+02 \cdot 27000+02$ $.27000+02 \cdot .27000+02$ $28000+02 \cdot 28000+02$ $.28000+02 \cdot 27000+02$ $.28000+02 \cdot 28000+02$ $.28060+02 \cdot 27000+02$. $.28000+02.28000+02$. $.28000+02 \cdot 28000+02$ $2800 \mathrm{C}+02.28 \mathrm{COC}+02$. $.28000+02 \cdot 28000+02$ $.29000+02 \cdot 28000+02$ $.29000+02 \cdot 29000+02$ $29000+02 \cdot 29000+02$ $.29000+02 \cdot 29000+02$ $.29000+02 \cdot 30000+02$ $.30000+02 \cdot 30000+02$ $.30000+02 \cdot 36000+02$ $.30000+02.30000+02$ . $30000+02 \cdot 30000+02$ $.30000+02 \cdot 30000+02$ $.31000+02 \cdot 31000+02$ $.31000+02 \cdot 31000+02$ . $31000+02 \cdot 31000+02$ $.28000+02 \cdot 29000+02$ $\begin{array}{ll}27000+02 & \cdot 27000+02 \\ 27000+02 & \cdot 27000+02\end{array}$ $27000+02 \cdot 27000+02$ - $27000+02.27000+02$ $.27000+02 \cdot 27000+02$ $.27000+02 \cdot 27000+02$ $.27000+02 \cdot 27000+02$ $.27000+02 \cdot 27000+02$ $.27000+02 \cdot 27000+02$ $.27000+02 \cdot 27000+02$ $.27000+02 \cdot 27000+02$ $.27000+02 \cdot 27000+02$ $.27000+02 \cdot 27000+02$ $.27000+02 \cdot 27000+02$ $.27000+02 \cdot 27000+02$ $.28000+02 \cdot 27000+02$ $.28000+02 \cdot 28000+02$ $28000+02 \cdot 28000+02$ - $28000+02 \cdot 28500+02$ $.28000+02 \cdot 28000+02$ $28000+02 \cdot 28000+02$ $.28000+02 \cdot 28000+02$ $.28000+02 \cdot 28000+02$ $.29000+02 \cdot 29000+02$ . $29000+02 \cdot 29000+02$ $.29000+02 \cdot 29000+02$ . $29000+02 \cdot 25000+02$ - $29000+02 \cdot 30000+02$ . $36000+02 \cdot 30000+02$ $.30000+02 \cdot 3 c 000+02$ $.30000+02 \cdot 30000+02$ $.30000+02 \cdot 30000+02$ $.30000+02 \cdot 30000+02$ $.31000+02 \cdot 31000+02$ $.31000+02 \cdot 31000+02$ $.31000+02 \cdot 31000+02$
$.27000+02$ - $27000+02$ $.27000+02$ $.27000+02$ $.27000+02$ $.27000+02$ $.27000+02$ $.27000+02$ - $27000+02$ - $27000+02$ - $27000+02$ $.27000+02$ - $27000+02$ - $27000+02$ $.27000+02$ $.27000+02$ $.27000+02$ - $28000+02$ $.28000+02$ - $28000+02$ $.28000+02$ - $28000+02$ - $28000+02$ - $28000+02$ - $29000+02$ - $29000+02$ - $29000+02$ $.2900 \mathrm{C}+\mathrm{C} 2$ - $29000+02$ . $30000+02$ - $30000+02$ -30000+02 $.30000+02$ . $30000+02$ - $31000+02$ - $31000+02$ $.31000+02$ 
Table 2. Data Acquisition System Formats, conto VIDAR AUTODATA EIGHT

SPEED2 Data Acquistition System Format Specification

INPUT $=C A R D$ I MAGES

READING $=(N)(K) 3 *(C)(K)(R .0) 5 *(R) 2 *(A)(K)$

TIME $=2 *(H)(K:) 2 *(M) 75 *(K)$

$E O R=80 *(K)$

$E C F=(K F)(K I)(K L)(K E)(K N)(K D)$

$05: 24$

5105

$0027.6<5106$

0030.0 C 5107

$0028.3 C 5108$

$0030.2 \mathrm{C}$

5109

0441.0 C 1110

$010.52 M V 1111$

$000.87 M V 2112$

$0.0984 \mathrm{~V}$

$05: 25$

5105

5109

0027.3 C 51060030.2 C 5107

0027.3 C 51060030.2 C 5107

$0028.3 C 5108$

$0030.5 \mathrm{C}$

FILEND

$001.06 M V 2112$

$0.0994 \mathrm{~V}$ 
Table 2. Data Acquisition System Formats, cont. ESTERLINE ANGUS PD2OE4

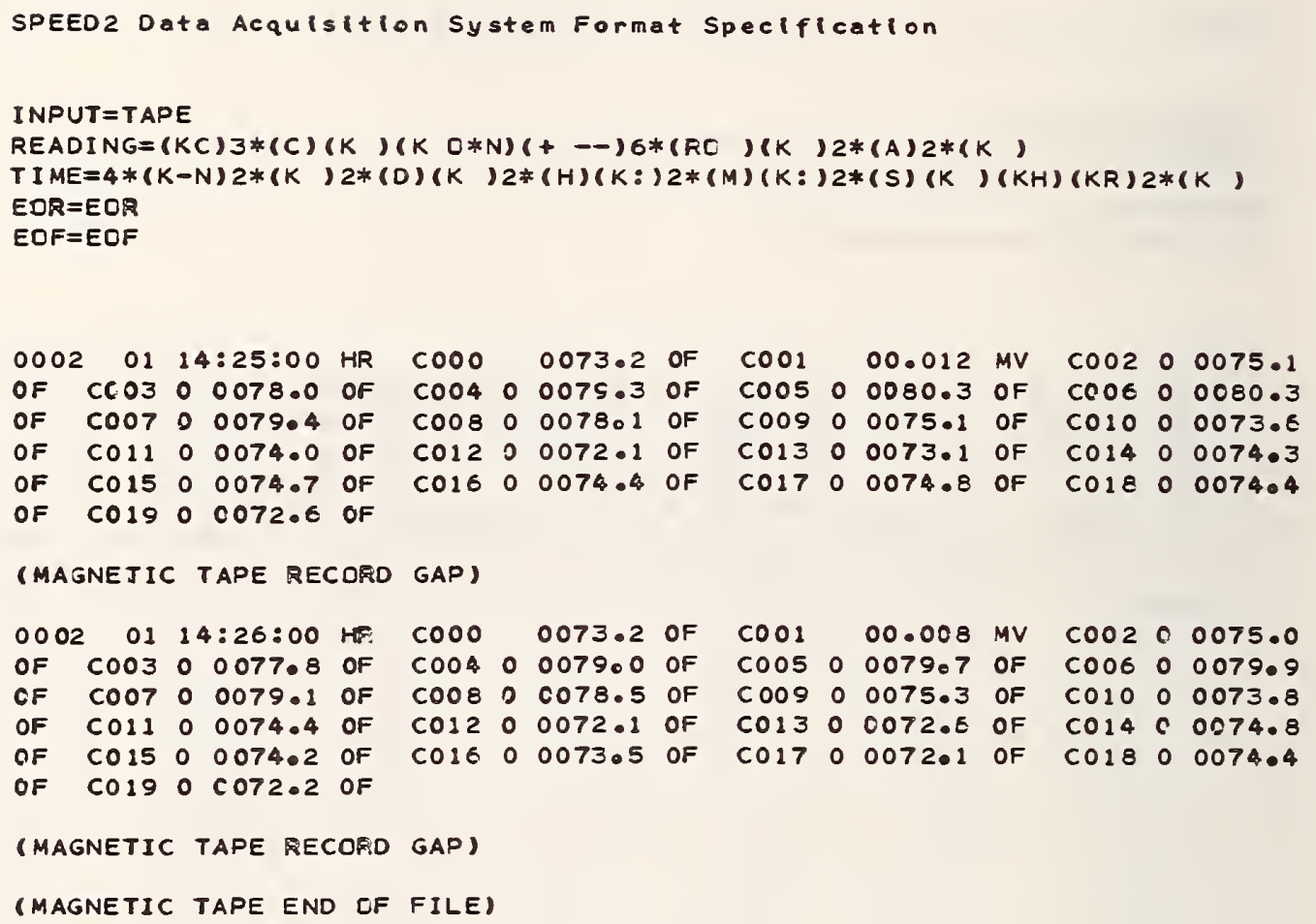


Table 2. Data Acquisition System Formats, cont. ESTERLINE ANGUS PD2C64

SPEED2 Data Acquisition System Format Specification

INPUT $=$ CARD IMAGES

READING $=(K C) 3 *(C)(K)(K D * N)(+--) 6 *(R D)(K) 2 *(A) 2 *(K)$

TIME $=4 *(K-N) 2 *\left(K^{*}\right) 2 *(D)(K) 2 *(H)(K:) 2 *(M)(K:) 2 *(S)(K)(K H)(K R) 2 *(K)$ $E O R=(K)(K X)$

$E O F=(K F)(K I)(K L)(K E)(K N)(K D)$

\begin{tabular}{|c|c|c|c|c|c|c|c|c|c|c|c|c|c|c|c|}
\hline 0002 & 01 & & $0: 25: 00$ & HR & $c 000$ & & 0073.2 & OF & $\cos 1$ & & 00.012 & MV & $\mathrm{COO2}$ & 0 & 0075.1 \\
\hline OF & $c 003$ & 0 & 0078.0 & OF & $\mathrm{COO4}$ & 0 & 0079.3 & OF & c005 & 0 & 0080.3 & OF & 1006 & 0 & 0080.3 \\
\hline OF & 1007 & 0 & 0079.4 & OF & $c 008$ & 0 & 0078.1 & OF & 1009 & 0 & $007 E \bullet 1$ & OF & $\cos 0$ & 0 & 0073.6 \\
\hline OF & $\cos 11$ & 0 & 0074.0 & OF & $\mathrm{CO} 12$ & 0 & 0072.1 & OF & $\operatorname{col} 3$ & 0 & 0073.1 & OF & $\mathrm{COI} 4$ & 0 & 0074.3 \\
\hline OF & Co 15 & 0 & 0074.7 & OF & $\operatorname{col} 6$ & 0 & 0074.4 & OF & $\mathrm{CO} 17$ & 0 & 0074.8 & OF & $\operatorname{col} 18$ & 0 & 0074.4 \\
\hline OF & $\operatorname{col} 19$ & 0 & 0072.6 & of & $x$ & & & & & & & & & & \\
\hline 0002 & 01 & 14 & $: 26: 00$ & HR & $\mathrm{COOO}$ & & 0073.2 & OF & $\mathrm{COOI}$ & & 00.008 & MV & $\mathrm{COO}_{2}$ & 0 & 0075.0 \\
\hline OF & $C 003$ & 0 & 0077.8 & OF & 1004 & 0 & 0079.0 & OF & $c 005$ & 0 & 0079.7 & of & 1006 & 0 & 0079.9 \\
\hline OF & $\mathrm{COOT}$ & 0 & 0079.1 & OF & $c 008$ & 0 & 007805 & of & 1009 & 0 & 0075.3 & OF & COL 10 & 0 & 0073.8 \\
\hline OF & $\cos 11$ & 0 & 0074.4 & OF & $\operatorname{col} 12$ & 0 & 0072.1 & OF & $\operatorname{col} 3$ & 0 & 0072.6 & OF & $C O 14$ & 0 & 0074.8 \\
\hline OF & $\operatorname{col} 15$ & 0 & 0074.2 & OF & $\operatorname{col} 16$ & 0 & 0073.5 & OF & $\mathrm{CO} 17$ & 0 & 0072.1 & OF & $\cos 18$ & 0 & 0074.4 \\
\hline OF & Co19 & 0 & 0072.2 & OF & $x$ & & & & & & & & & & \\
\hline
\end{tabular}
FILEND 
Table 2. Data Acquisition System Formats. cont. VIDAR AUTODATA NINE

SPEED2 Data Acquisition System Format Specification

INPUT $=$ TAPE

RE ADING $=3 *(C)(K)(t+--0) 5 *(V O)(V K) 3 *(A)(E 0)(K X)$

TIME $=3 *(D) 2 *(H)(K:) 2 *(M)(K:) 2 *(S) 4 *(K)(K X)$

$E Q R=E Q R$

$E O F=E D F$

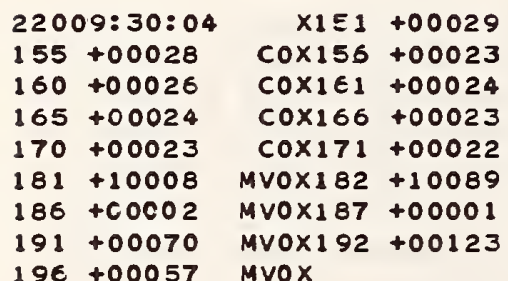

(MAGNETIC TAPE RECORD GAP)

$22009: 30: 19$

$155+00060$

$160+00025$

$165+00024$

$170+00023$

$181+10001$

$186+00002$

$191+00082$

$196+00036$

$\times 151+00034$ cox156+00022 $\operatorname{cox} 161+00024$ $\operatorname{cox} 166+00023$ $\operatorname{cox} 171+00022$ $M V 0 \times 182+10078$ MVOX187+00000 MVOX192+00130 MvoX

(MAGNETIC TAPE RECORD GAP)

(MAGNETIC TAPE END OF FILE)

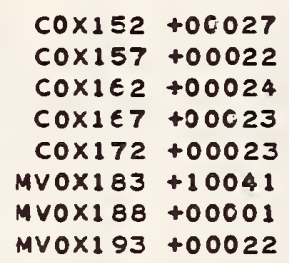

cox152+00031 c0X157+00022 cox1E2 +00024 C $0 \times 167+00023$ C0X172+00022 MVox183+10035 MVOX $188+00000$ $M \vee 0 \times 193+00012$ $\operatorname{cox} 153+0002 \epsilon$ $\operatorname{cox} 158+00022$ $\operatorname{cox} 163+00024$ COX $1 \in 8+C 0023$ Cox173+0002E MVOX184+10073 $M V O X 189+00000$ MVOX194-00004

Cox153+00033 Cox158+00022 $\operatorname{cox} 163+00024$ Cox168+00023 $\operatorname{cox} 173+0002 \epsilon$ MVOX184+10068 MVOX $189+00001$ $M V O \times 194+00039$ cox $154+00028$ cox159+00022 $\operatorname{cox} 164+00023$ coxicg +00024 C0X180 +09874 MVOX185+10321 MVOX190+00001 MVOX195 -00007

C0X154+00047 cox159+00022 $\operatorname{cox} 164+00023$ $\operatorname{cox} 169+00024$ CoX180 +09857 MVOX185+10320 MV0X190+00000 MVoX195 -00005 $\operatorname{cox}$ $\operatorname{cox}$ $\operatorname{cox}$ $\operatorname{cox}$ MVOX MVOX MVOX MVOX

$\operatorname{cox}$ $\operatorname{cox}$ $\operatorname{cox}$ $\operatorname{cox}$ $\operatorname{mvox}$ MVOX MVOX Mvox 
Table 2. Data Acquisition System Formats. cont. VIDAR AUTODATA NINE

SPEEO2 Data AcQuisition System Format Specification

INPUT $=$ CARD I MAGES

READING $=3 *(C)(K)(++--0) 5 *(V O)(V K) 3 *(A)(E D)(K X)$

TIME $=3 *(D) 2 *(H)(K:) 2 *(M)(K:) 2 *(S) 4 *(K)(K X)$

$E D R=(K)(K X)$

$E O F=(K F)(K I)(K L)(K E)(K N)(K D)$

$22009: 30: 04$
$155+00028$
$160+00026$
$165+00024$
$170+00023$
$181+10008$
$186+00002$
$191+00070$
$196+00057$
$22009: 30: 19$
$155+00060$
$160+00025$
$165+00024$
$170+00023$
$181+10001$
$186+00002$
$191+00082$
$196+00036$
$F I L E N D$

$22009: 30: 04$

$\times 151+00029$

Cox156+00023

$\operatorname{cox} 161+00024$

c0X166+00023

$\operatorname{cox} 171+00022$

MVOX182+10089

MVOX187+00001

MVOX192+00123 $\operatorname{MvOX} \times$

$x 151+00034$

$\operatorname{cox} 156+00022$

Cox $1 \epsilon 1+00024$

Cox $166+00023$

$\operatorname{cox} 171+00022$

MVOX182+10078

$M V O X 187+00000$

MVoX192+00130

$\operatorname{mvox} x$ cox152+00027 $\operatorname{cox} 157+00022$ coxie2 +00024 COX1E7 +00023 $\operatorname{cox} 172+00023$ $M V O X 183+10041$ MVOX188+00C01 MVOX193+00022

Cox152+00031 cox157+00022 $\operatorname{cox} 162+00024$ COX1E7 +00023 $\operatorname{cox} 172+00022$ MVOX183+10035 MVOX188 +00000 MVOX193+00012

\begin{tabular}{|c|c|}
\hline $\operatorname{cox} \times 153$ & +00026 \\
\hline $\operatorname{cox} 158$ & +00022 \\
\hline $\operatorname{cox} \times 163$ & +00024 \\
\hline $\operatorname{cox} 168$ & +00023 \\
\hline $\operatorname{cox} \times 173$ & +00026 \\
\hline MVO X1 84 & +10073 \\
\hline MVOX189 & +00000 \\
\hline MVOX 194 & -00004 \\
\hline $\operatorname{cox} \times 153$ & +00033 \\
\hline $\operatorname{cox} \times 158$ & +00022 \\
\hline $\operatorname{cox} \times 163$ & +00024 \\
\hline $\operatorname{cox} \times 168$ & +00023 \\
\hline $\operatorname{cox} 173$ & +00026 \\
\hline MVOX 184 & +10068 \\
\hline MVOX $\times 189$ & +00001 \\
\hline MVOX 19 & +0003 \\
\hline
\end{tabular}

$\operatorname{cox} 154+00028$ $\operatorname{cox} 159+00022$ C $0 \times 164+00023$ Coxieg +00024 C0X180+09874 MVOX185+10321 MVOX190+00001 MVOX155 -00007

C0X154+00047 cox159+00022 cox $164+00023$ $\operatorname{cox} 169+00024$ CoX180+09857 MVOX185+10320 MVOX $190+00000$ MVOX195-00005 $\operatorname{cox}$

$\operatorname{cox}$ $\cos$ $\operatorname{cox}$ MvoX MVOX MvoX MVOX

$\operatorname{cox}$ $\operatorname{cox}$ $\operatorname{cox}$ $\operatorname{cox}$ $\operatorname{Mvox}$ Mvox Mvox MvoX 


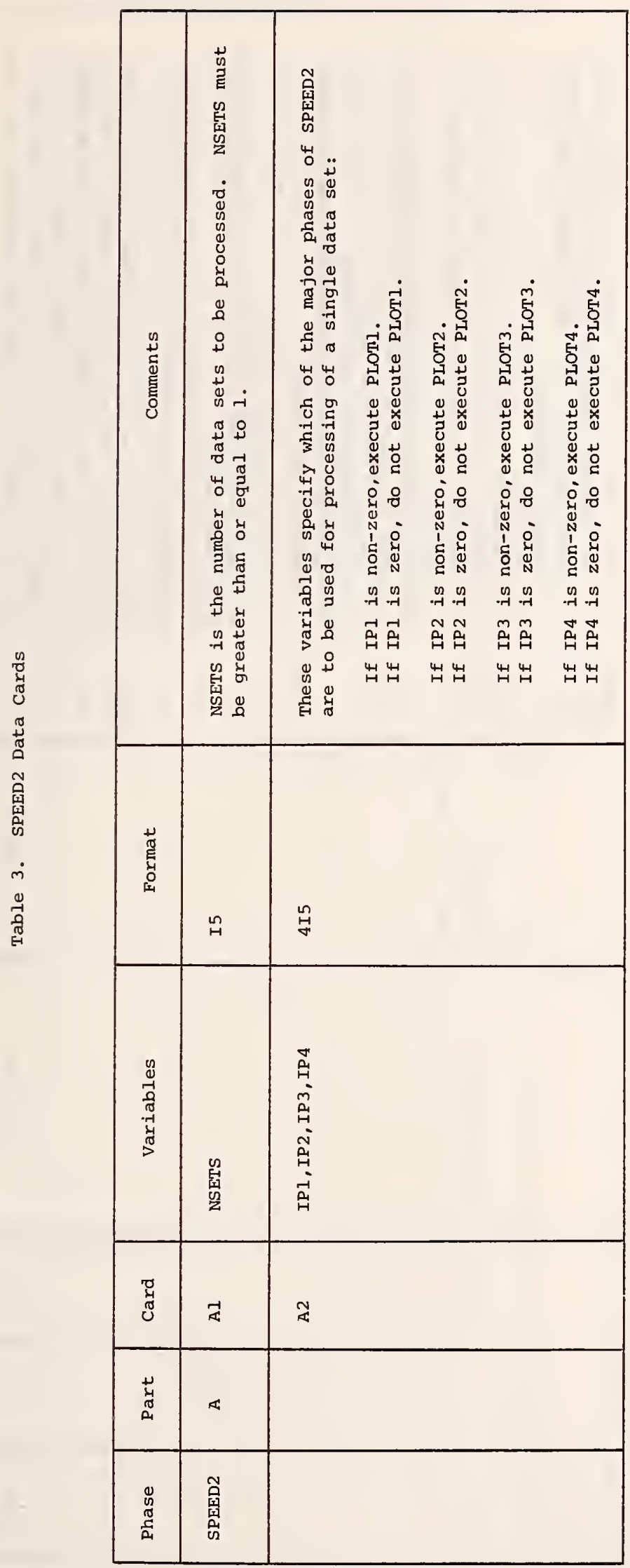




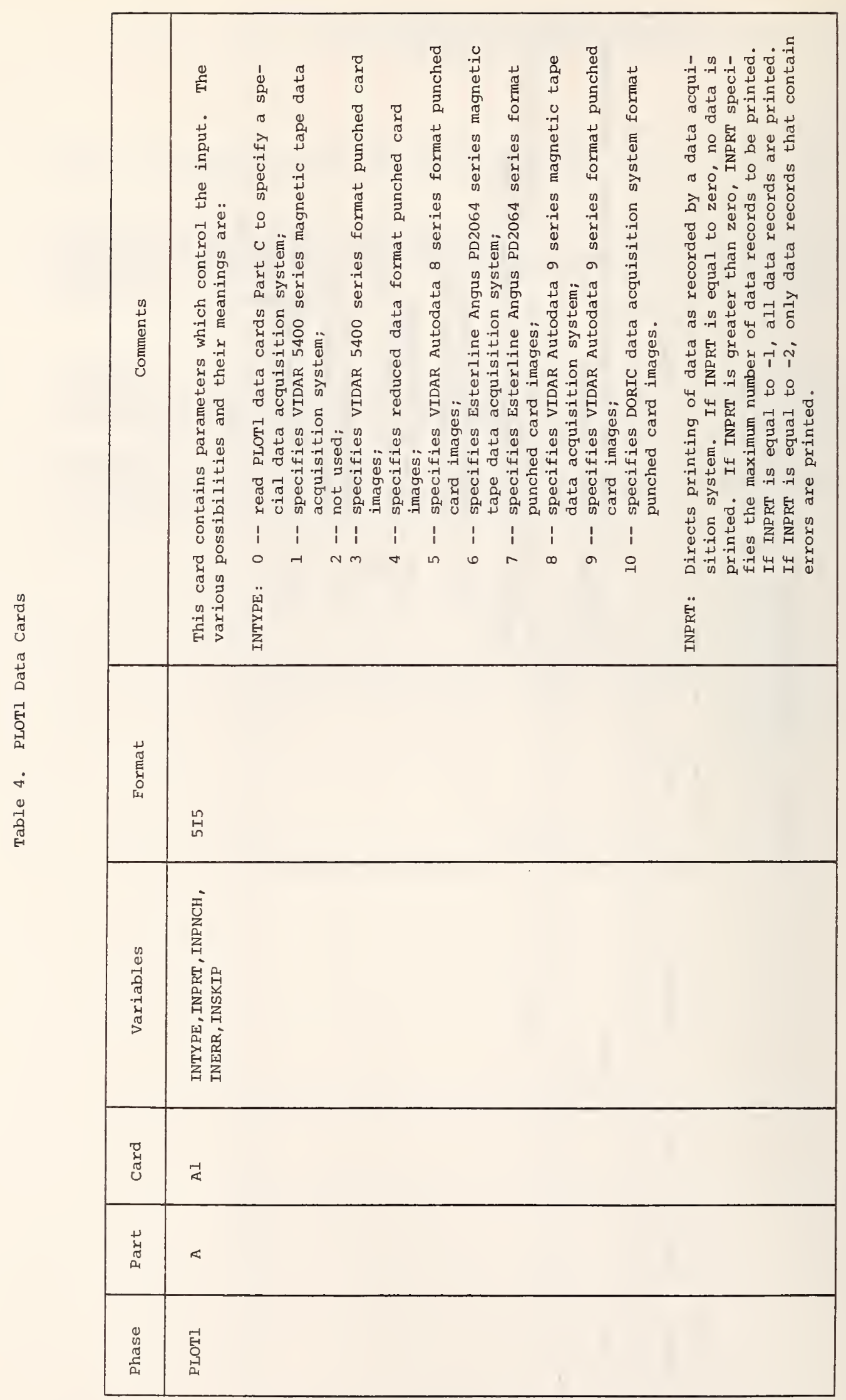




\begin{tabular}{|c|c|c|c|c|c|}
\hline 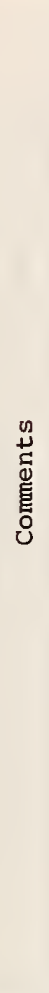 & 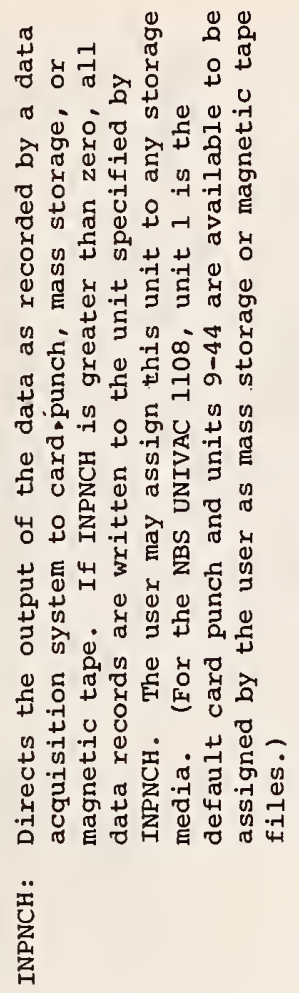 & 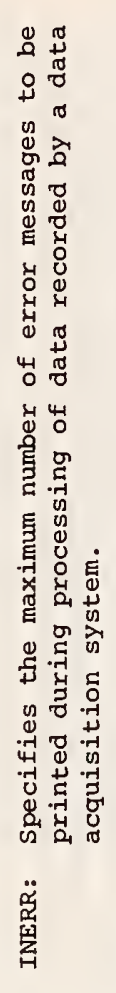 & 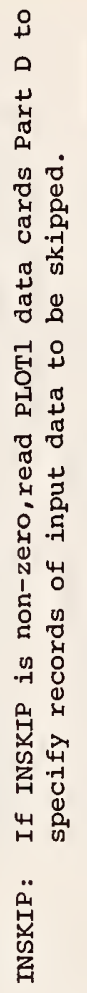 & 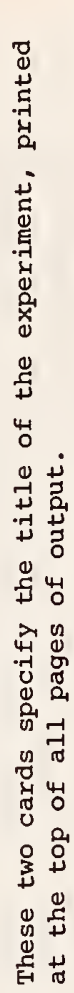 & 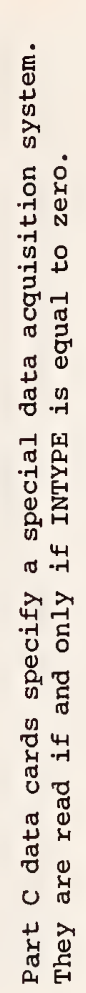 \\
\hline 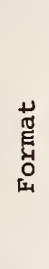 & & & & 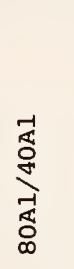 & \\
\hline 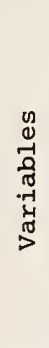 & & & & 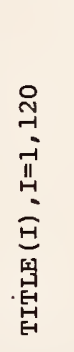 & \\
\hline ర్ర & & & & $\stackrel{\mathscr{M}}{\stackrel{N}{m}}$ & \\
\hline 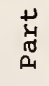 & & & & Ф & $u$ \\
\hline 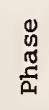 & & & & 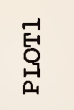 & 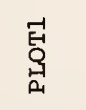 \\
\hline
\end{tabular}




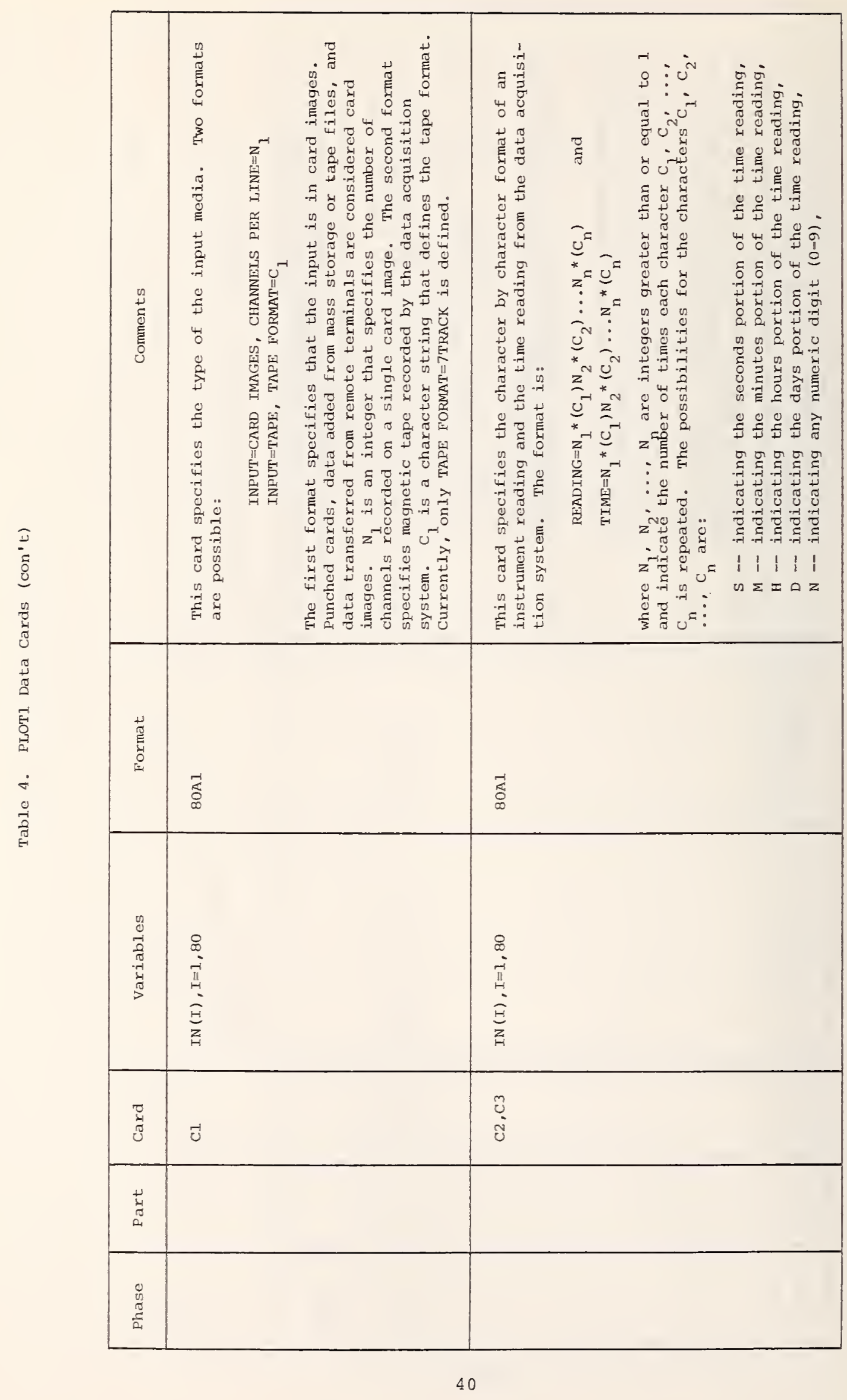




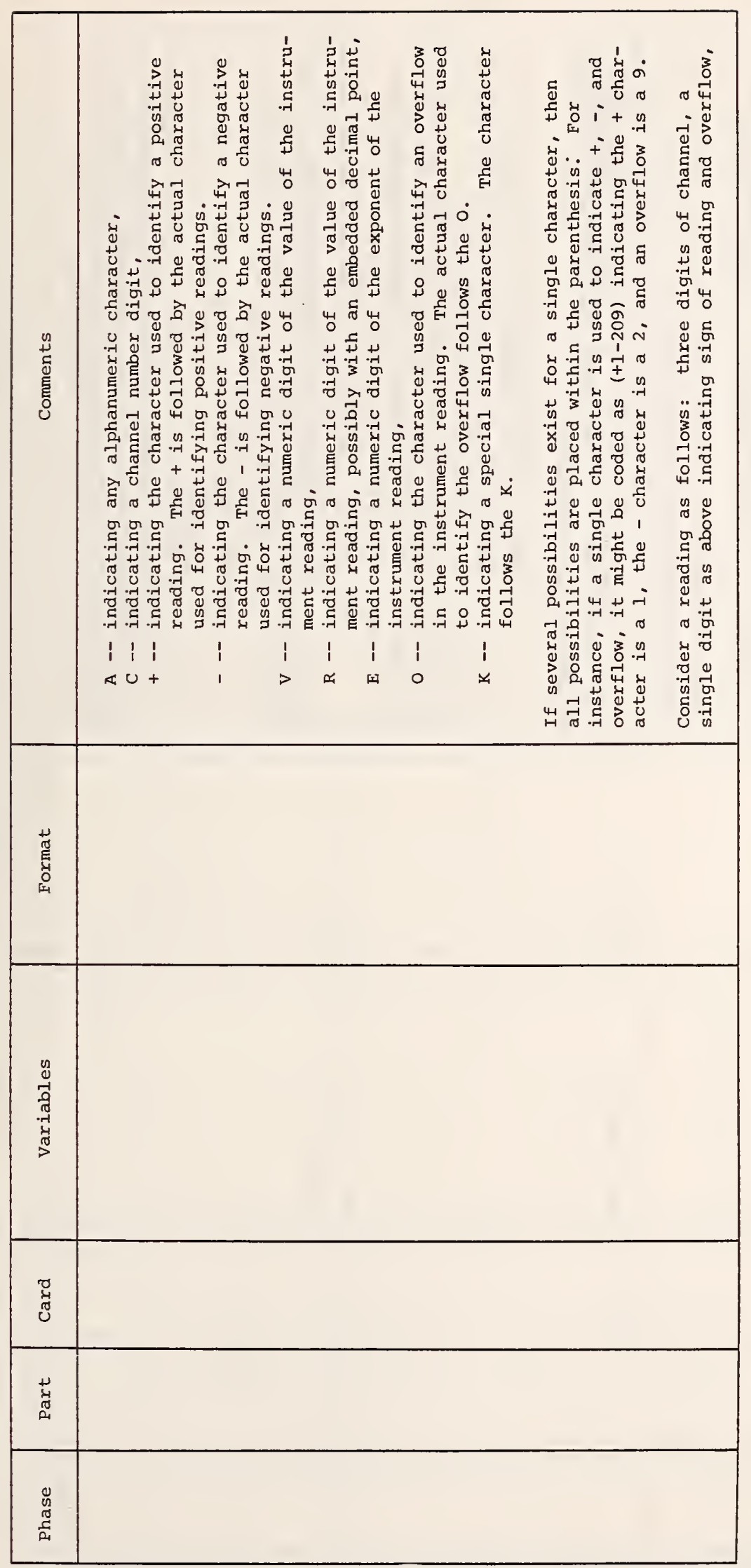




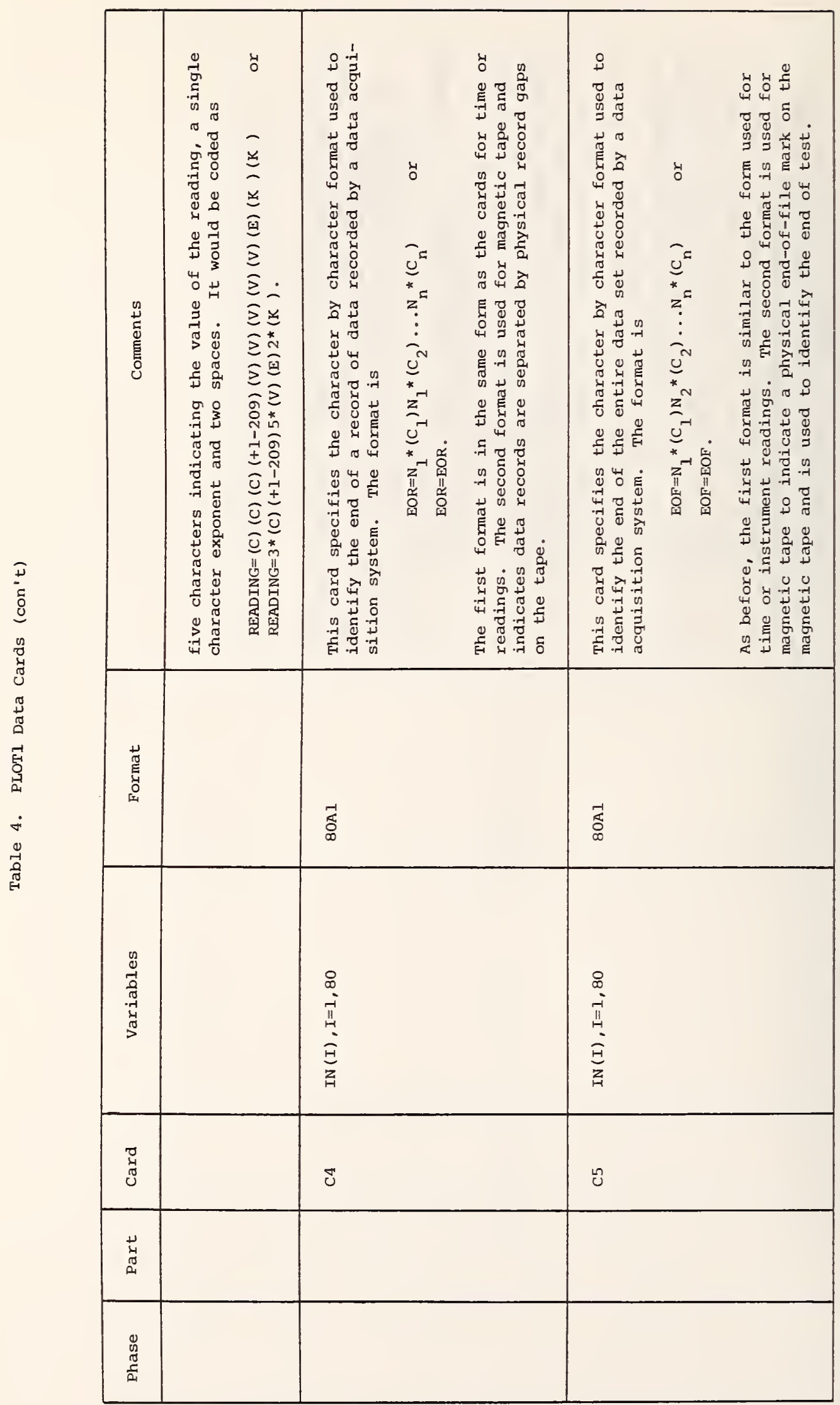




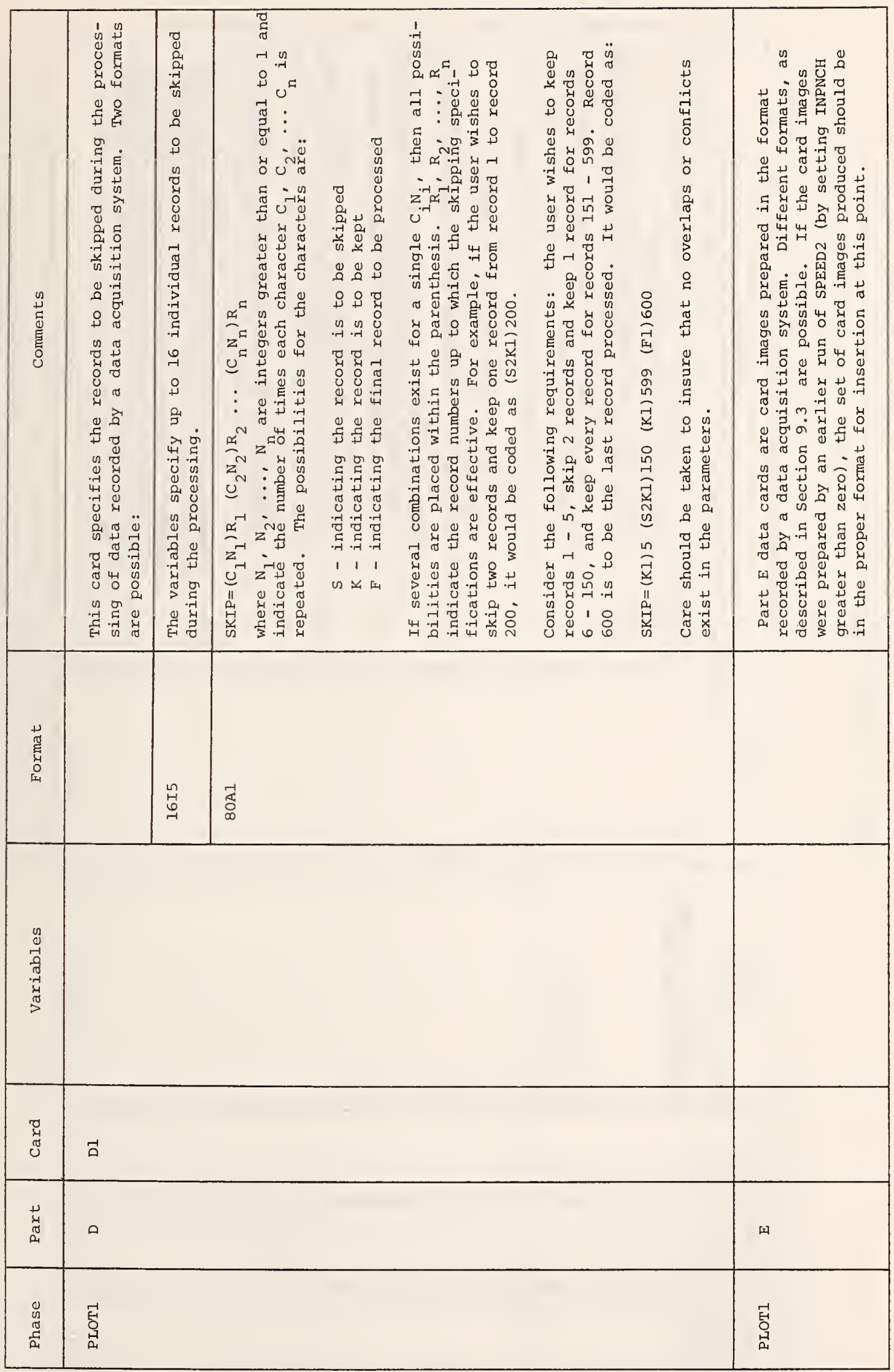




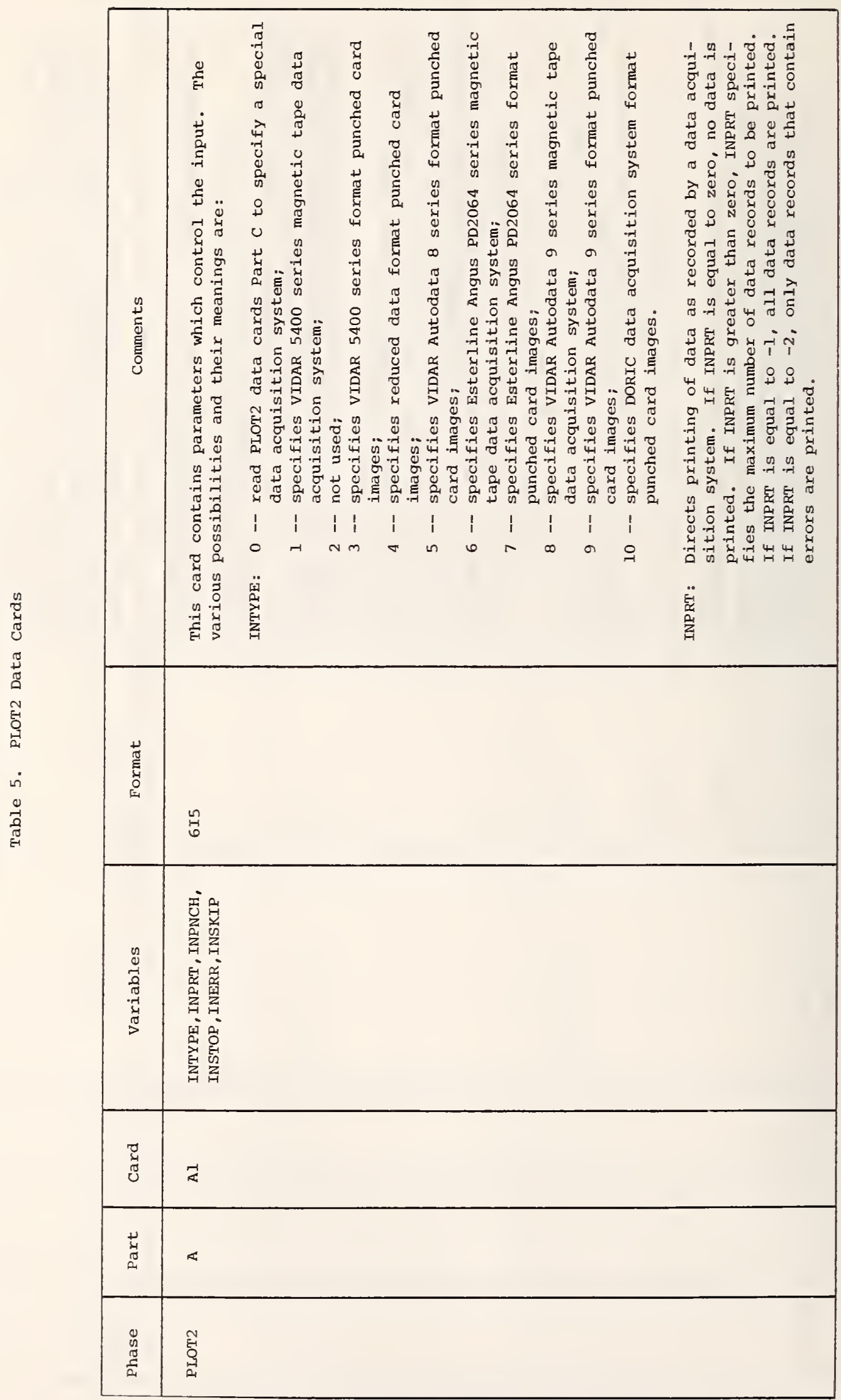




\begin{tabular}{|c|c|c|c|c|c|c|}
\hline 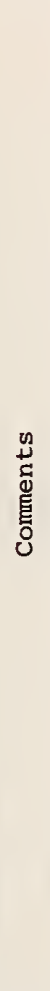 & 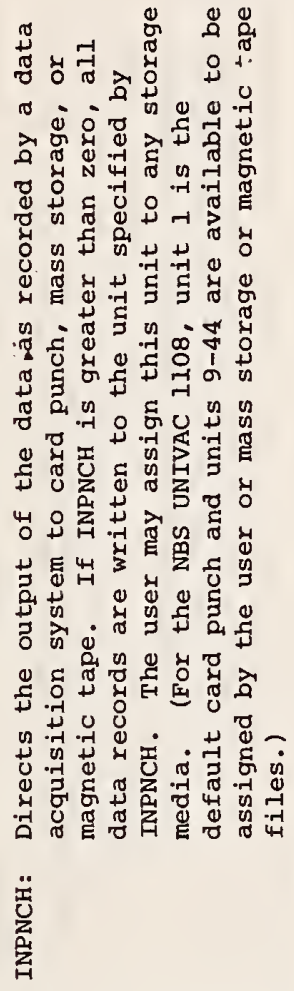 & 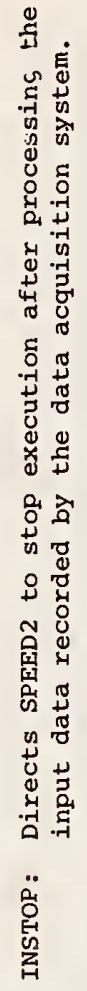 & 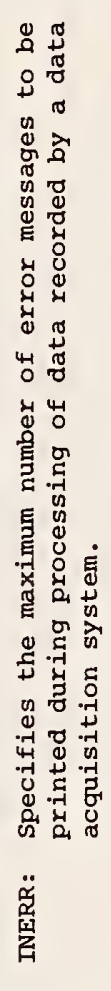 & 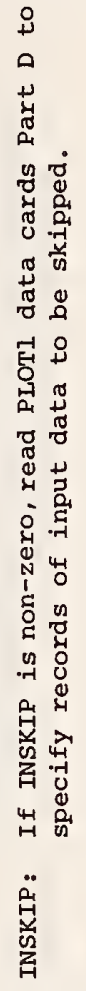 & 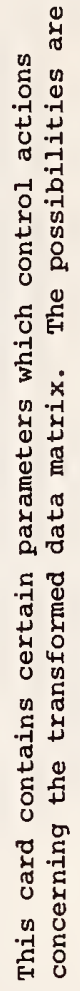 & 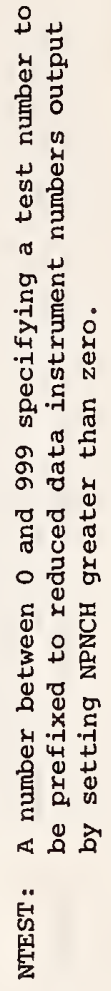 \\
\hline 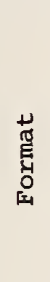 & & & & & 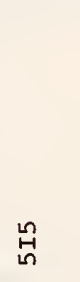 & \\
\hline $\begin{array}{l}0 \\
0 \\
-1 \\
-70 \\
-4 \\
0 \\
0\end{array}$ & & & & & 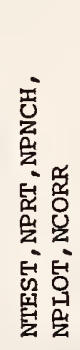 & \\
\hline 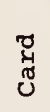 & & & & & $\tilde{\pi}$ & \\
\hline है & & & & & & \\
\hline 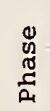 & & & & & & \\
\hline
\end{tabular}




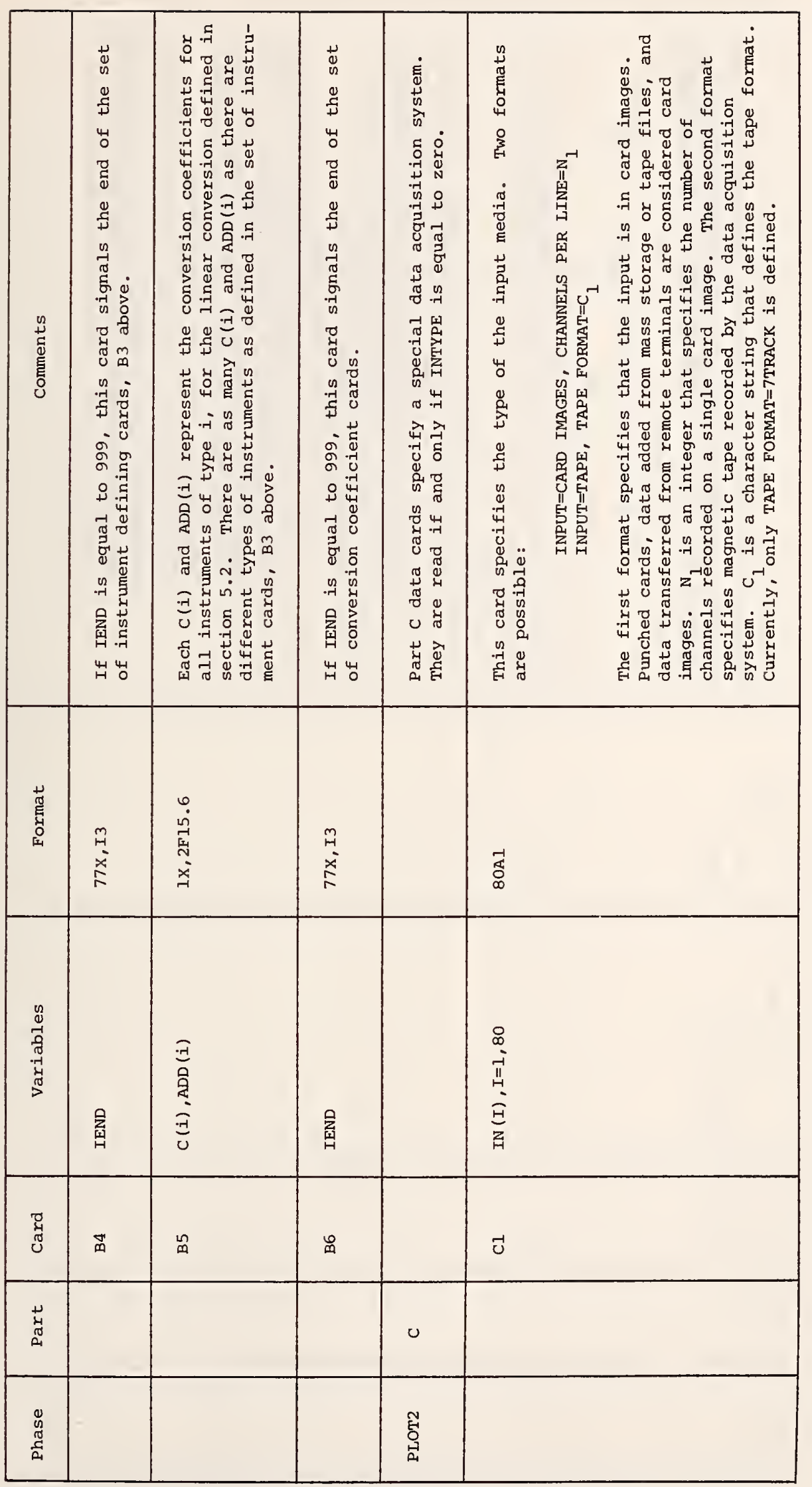




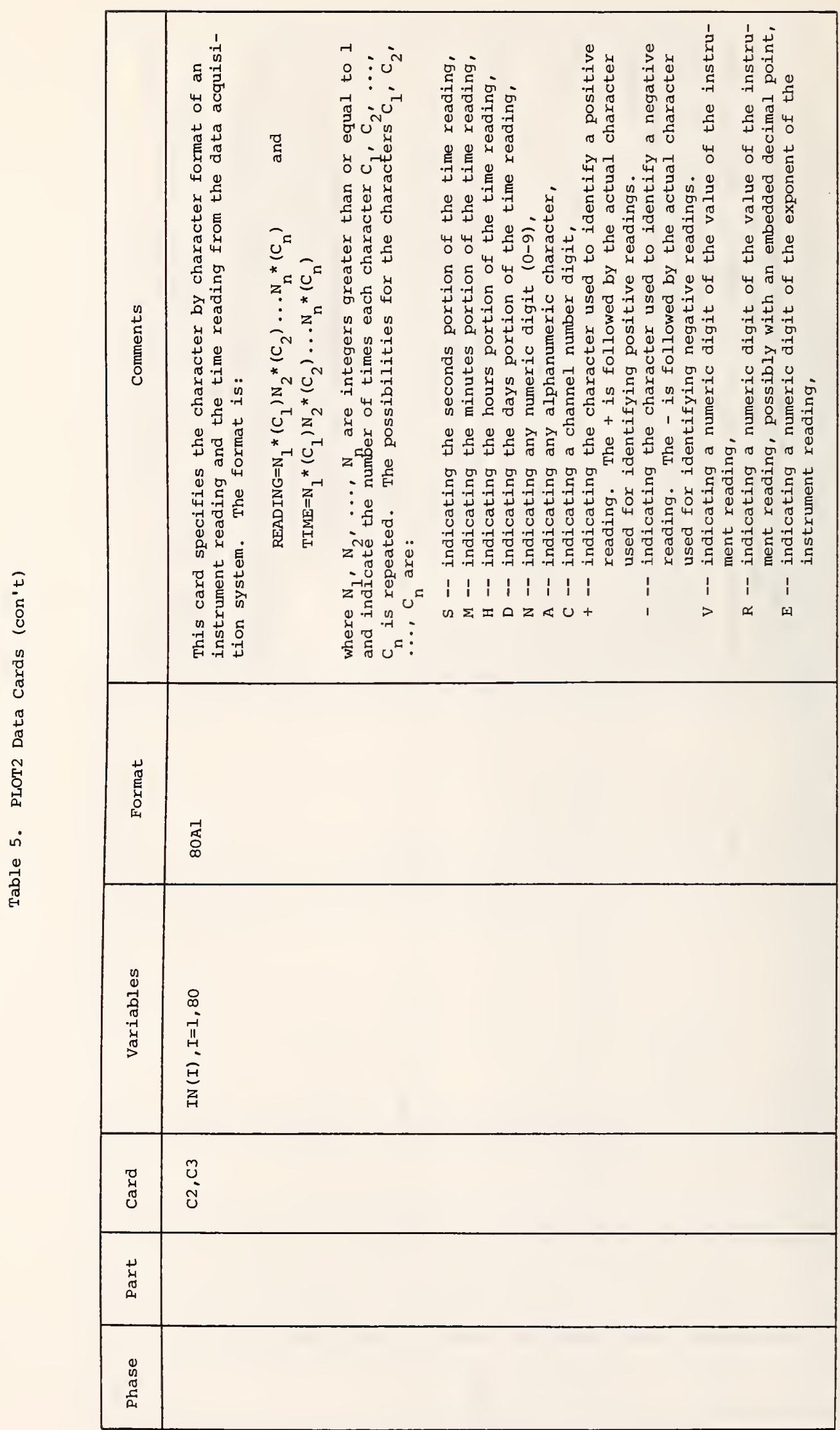




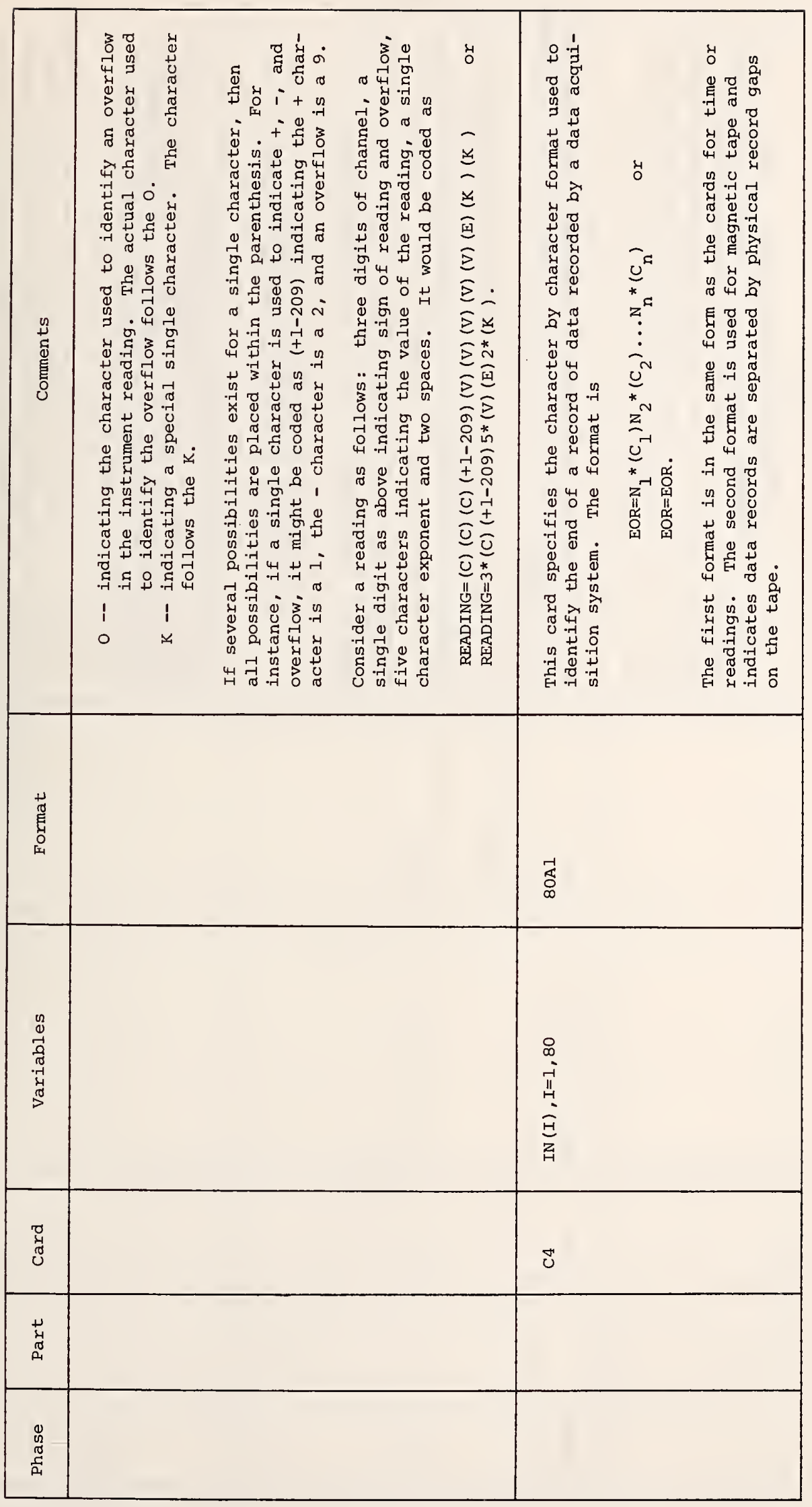




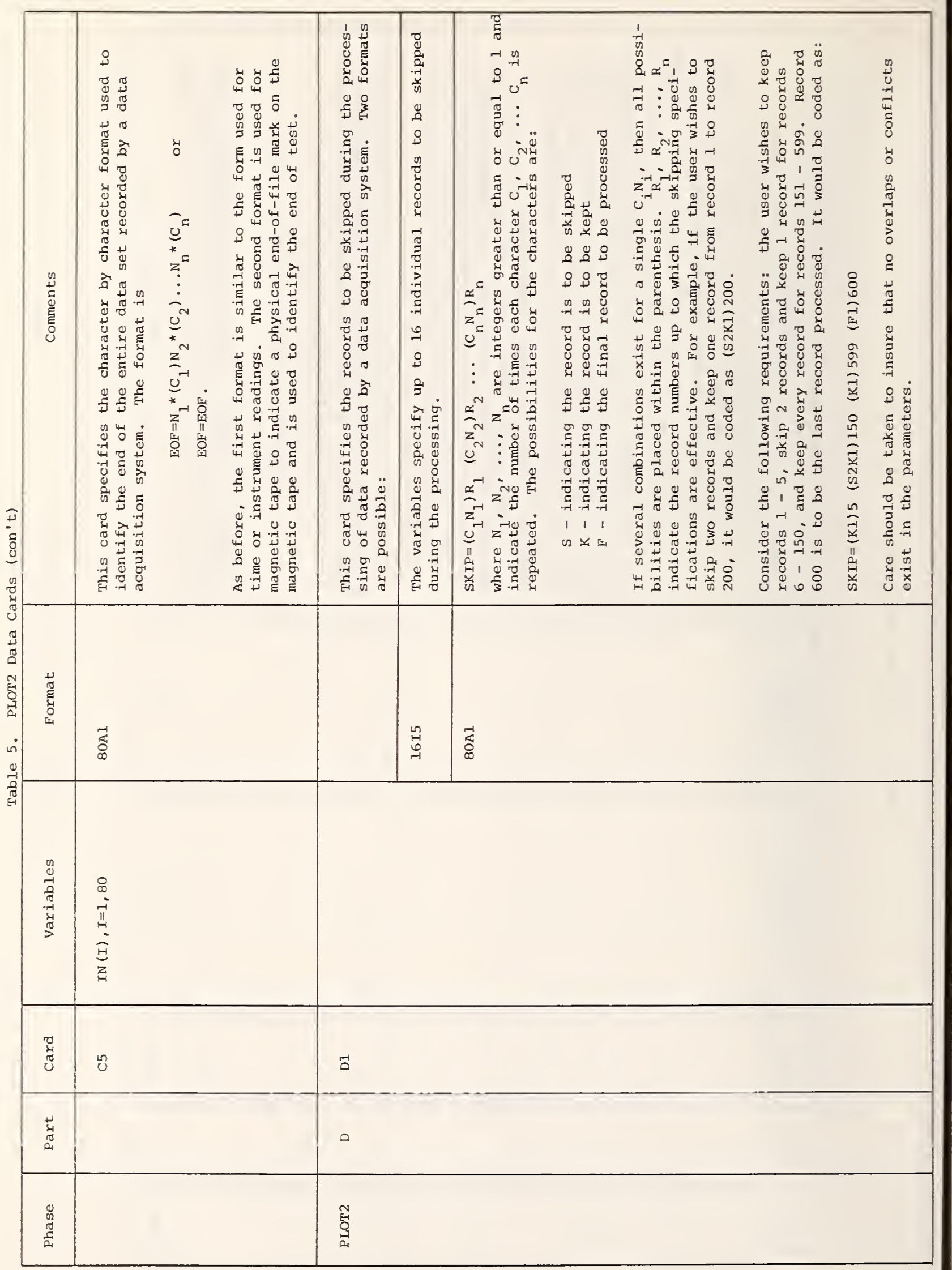




\begin{tabular}{|c|c|c|c|c|c|}
\hline 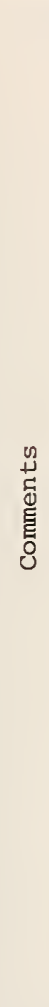 & 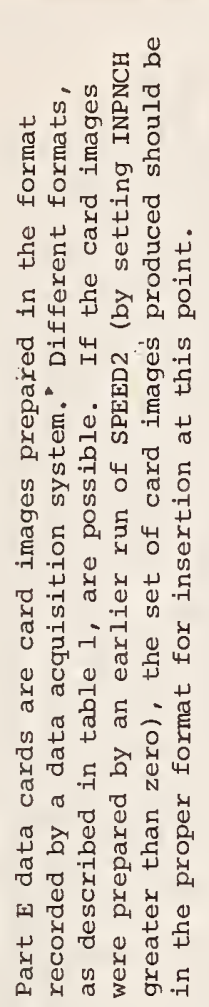 & 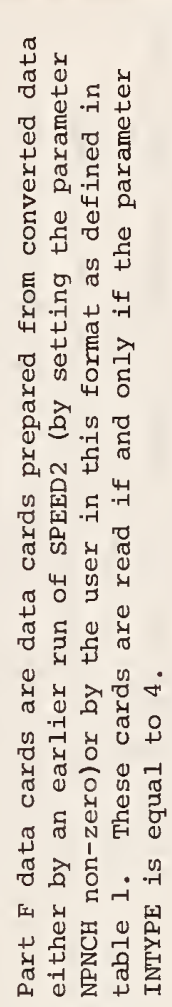 & 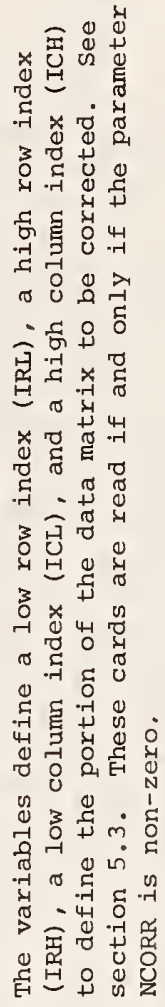 & 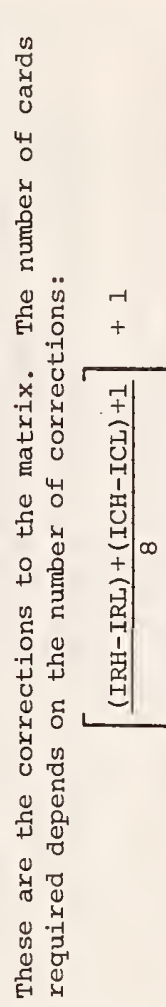 & 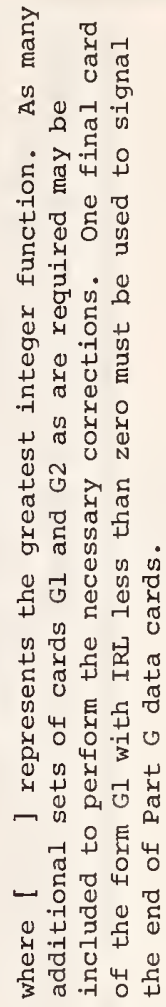 \\
\hline 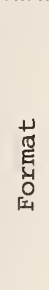 & & & $\stackrel{\stackrel{\sim}{H}}{\forall}$ & $\begin{array}{l}\circ \\
\dot{0} \\
\text { 師 }\end{array}$ & \\
\hline $\begin{array}{l}0 \\
0 \\
-1 \\
0 \\
-7 \\
-1 \\
\tilde{0} \\
0\end{array}$ & & & 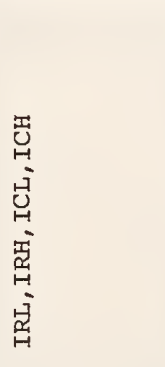 & 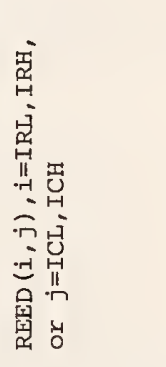 & \\
\hline $\begin{array}{l}\bar{y} \\
\tilde{J} \\
0\end{array}$ & & & - & లै & \\
\hline $\begin{array}{l}H_{y} \\
\sigma \\
\Omega_{1}\end{array}$ & Ф & 所 & 0 & & \\
\hline $\begin{array}{l}0 \\
\text { D } \\
\text { శ } \\
\text { صి }\end{array}$ & $\begin{array}{l}\text { 舁 } \\
\text { 只 } \\
\end{array}$ & $\begin{array}{l}\underset{⿴}{N} \\
\underset{\leftrightarrow}{\circ} \\
\mu_{1}\end{array}$ & 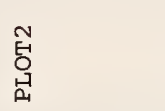 & & \\
\hline
\end{tabular}




\begin{tabular}{|c|c|c|c|c|c|c|}
\hline 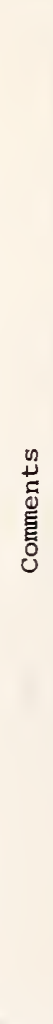 & 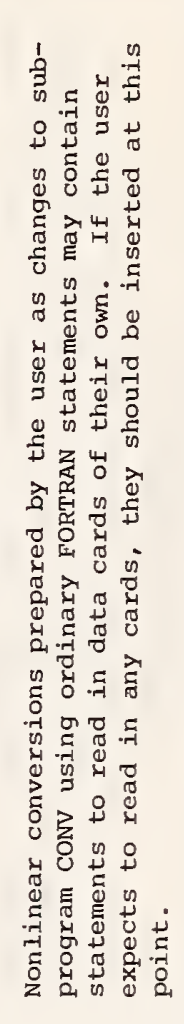 & 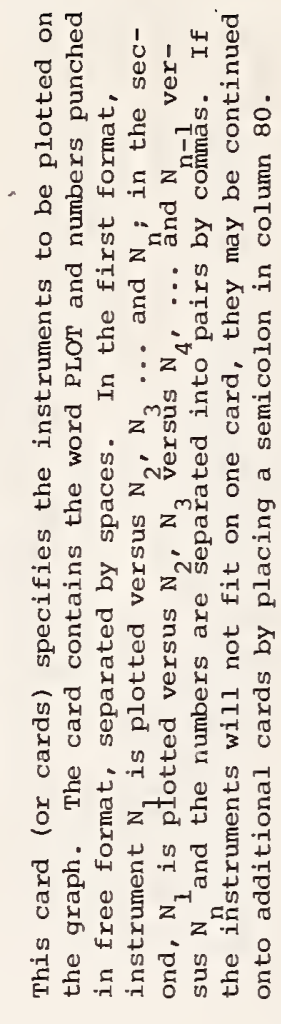 & 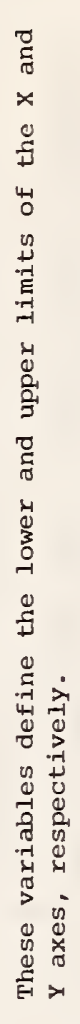 & 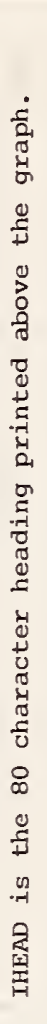 & 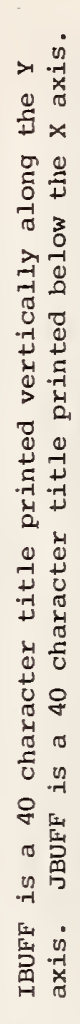 & 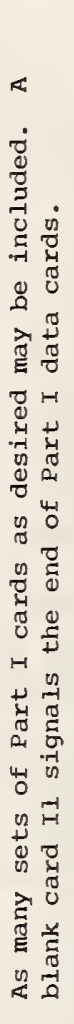 \\
\hline \multirow{2}{*}{ 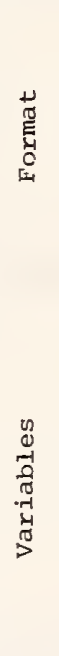 } & & \multirow{2}{*}{ 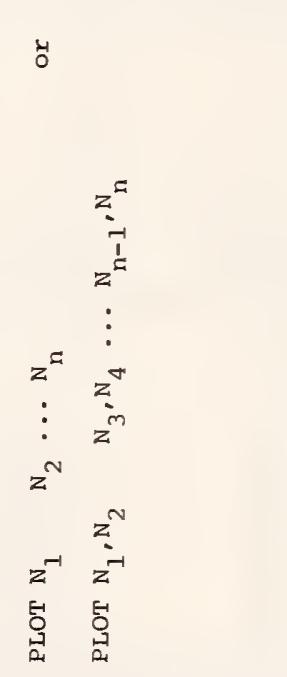 } & $\begin{array}{l}0 \\
\dot{0} \\
\stackrel{1}{8}\end{array}$ & $\underset{\infty}{\stackrel{1}{\delta}}$ & $\underset{\Phi}{\vec{\delta}}$ & \\
\hline & & & 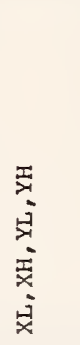 & 睍 & 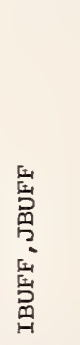 & \\
\hline $\begin{array}{l}\text { ర్ } \\
\text { లే }\end{array}$ & & $\stackrel{-1}{H}$ & $\underset{7}{\mathbb{7}}$ & $\stackrel{M}{H}$ & $\mathbb{H}$ & \\
\hline 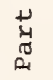 & $I$ & $H$ & & & & \\
\hline $\begin{array}{l}0 \\
\text { 足 } \\
\text { 足 } \\
\text { D. }\end{array}$ & 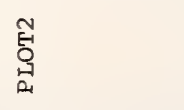 & $\begin{array}{c}\text { 舁 } \\
\text { 品 }\end{array}$ & & & & \\
\hline
\end{tabular}




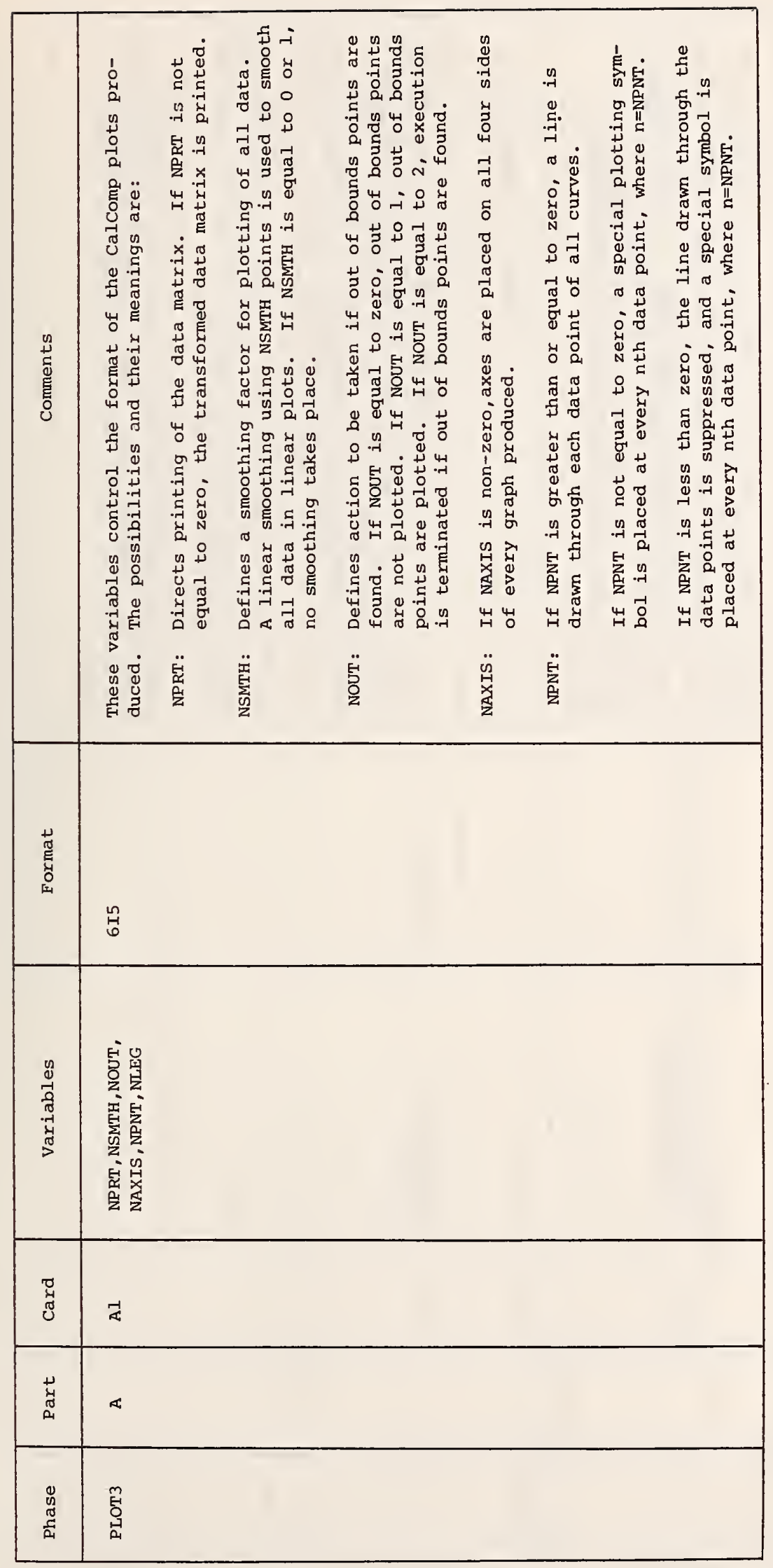




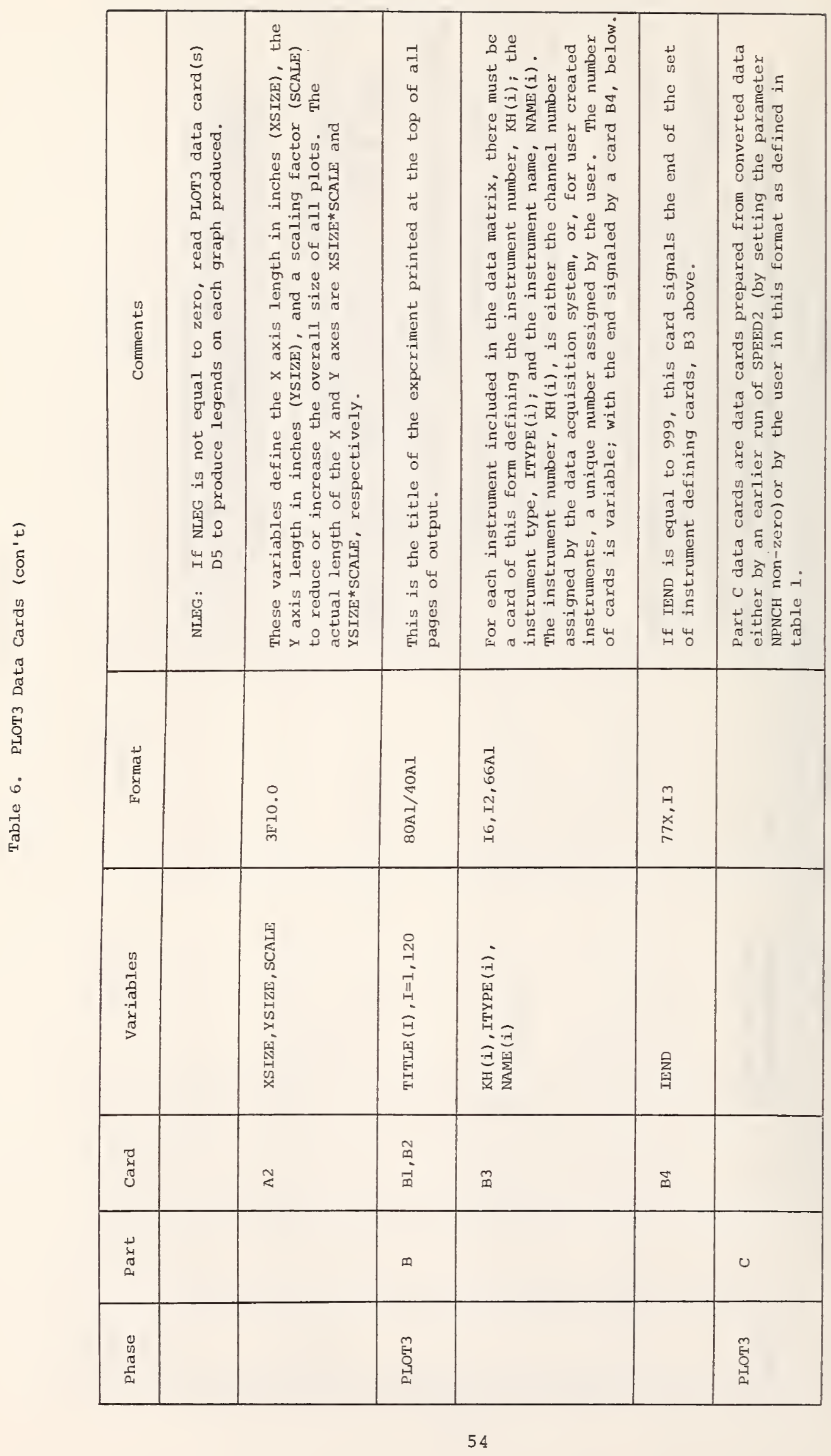




\begin{tabular}{|c|c|c|c|c|c|c|}
\hline 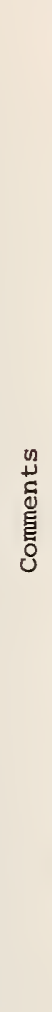 & 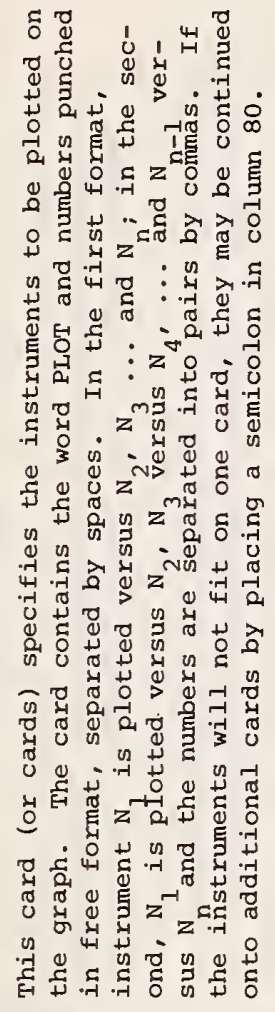 & 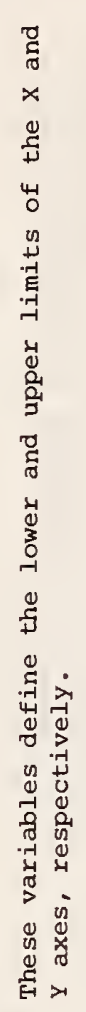 & 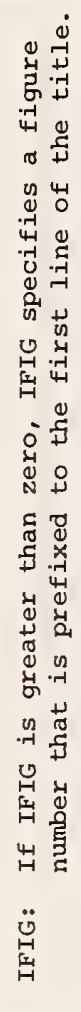 & 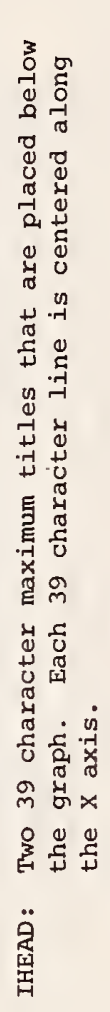 & 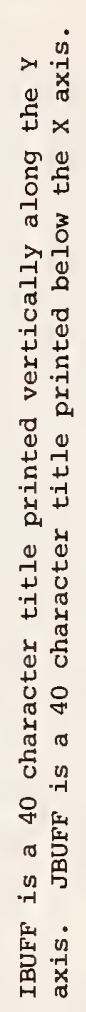 & 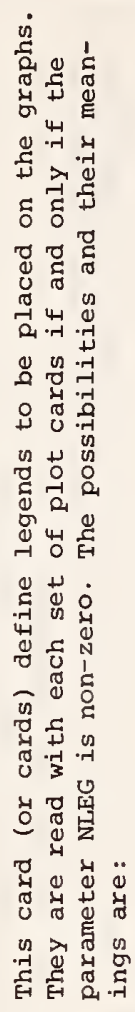 \\
\hline 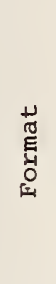 & $z^{a}$ & $\begin{array}{l}0 \\
\dot{0} \\
-1 \\
\dot{8}\end{array}$ & 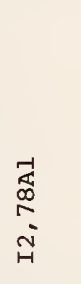 & & 灾 & 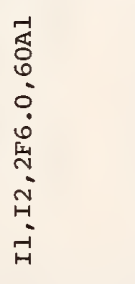 \\
\hline $\begin{array}{l}n \\
0 \\
-1 \\
0 \\
-\pi \\
-1 \\
\pi \\
\infty\end{array}$ & 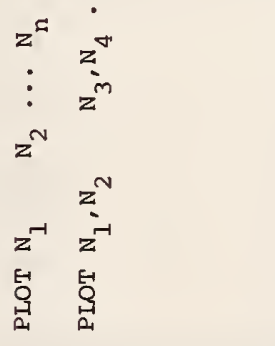 & 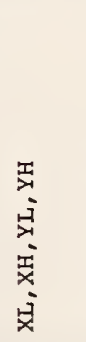 & 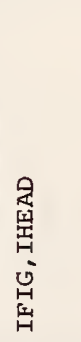 & & 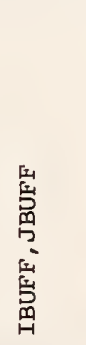 & 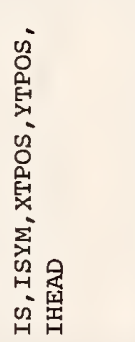 \\
\hline $\begin{array}{l}\text { '] } \\
\text { రુ }\end{array}$ & $\overrightarrow{0}$ & $\tilde{a}$ & $\hat{a}$ & & $\vec{\Delta}$ & $\stackrel{\llcorner}{\circ}$ \\
\hline 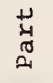 & 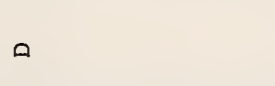 & & & & & \\
\hline $\begin{array}{l}0 \\
0 \\
\text { đ } \\
\text { a }\end{array}$ & $\begin{array}{l}\text { 舁 } \\
\text { 品 }\end{array}$ & & & & & \\
\hline
\end{tabular}




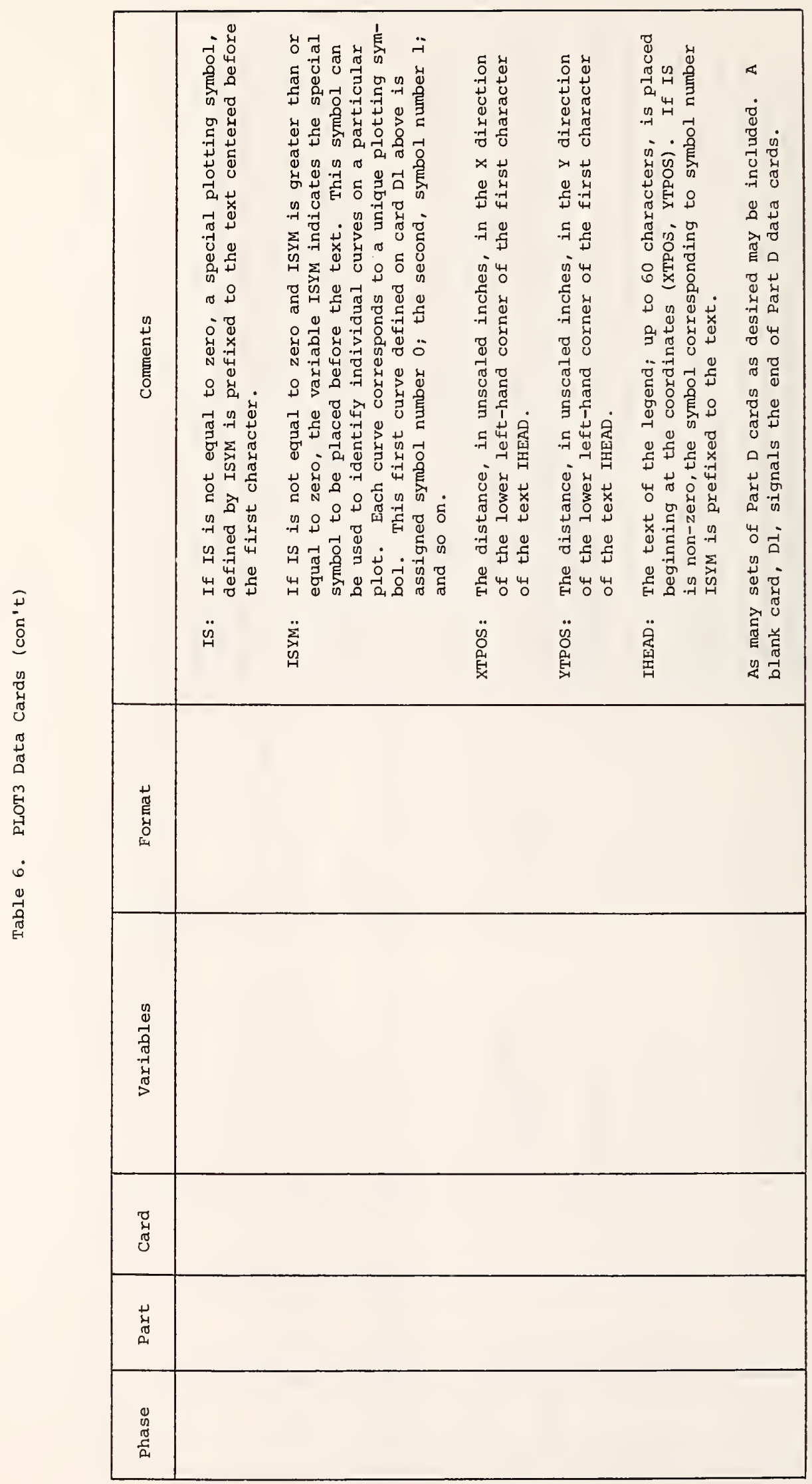




\begin{tabular}{|c|c|c|c|c|c|}
\hline 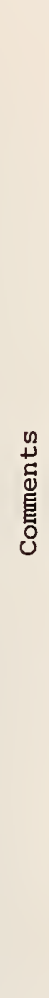 & 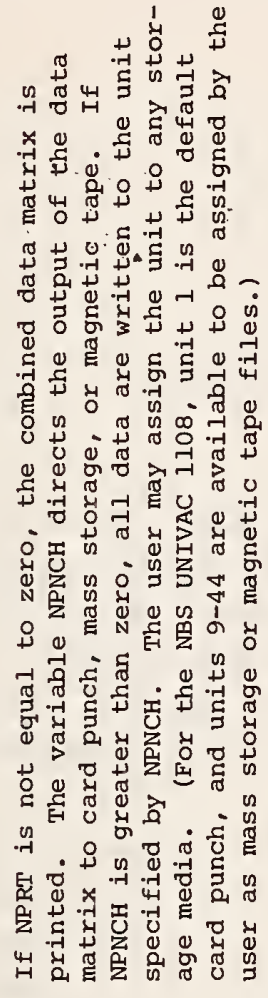 & 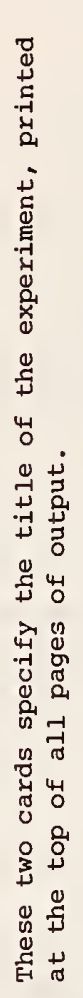 & 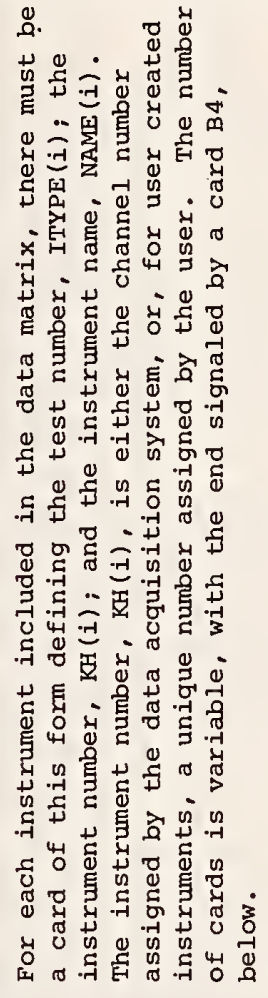 & 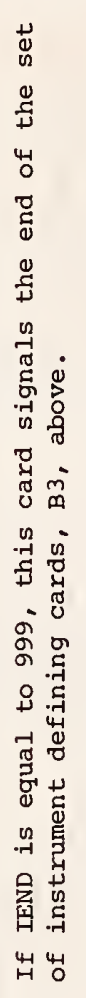 & 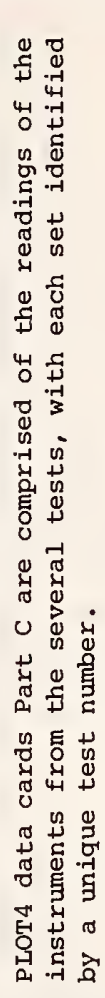 \\
\hline 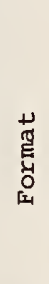 & $\stackrel{\mathscr{H}}{N}$ & 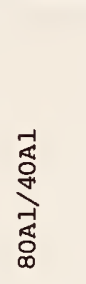 & 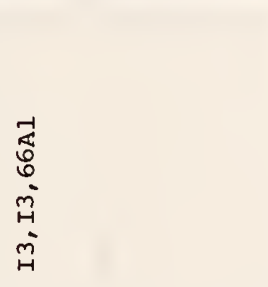 & $\begin{array}{l}\stackrel{m}{+} \\
\stackrel{x}{x}\end{array}$ & \\
\hline 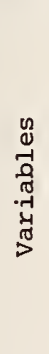 & 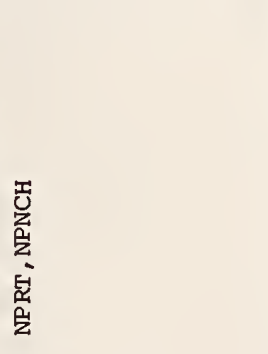 & ঙ & 离 & 音 & \\
\hline $\begin{array}{l}\overline{\text { T}} \\
\text { Uू }\end{array}$ & $\vec{a}$ & $\stackrel{m}{m}$ & $\stackrel{m}{m}$ & म̈ & \\
\hline 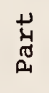 & 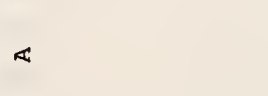 & $m$ & & & 0 \\
\hline $\begin{array}{l}0 \\
0 \\
0 \\
\tilde{0} \\
\tilde{0}\end{array}$ & $\begin{array}{l}\text { 范 } \\
\text { 吕 }\end{array}$ & 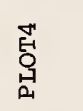 & & & $\begin{array}{l}\text { 范 } \\
\text { 吕 }\end{array}$ \\
\hline
\end{tabular}




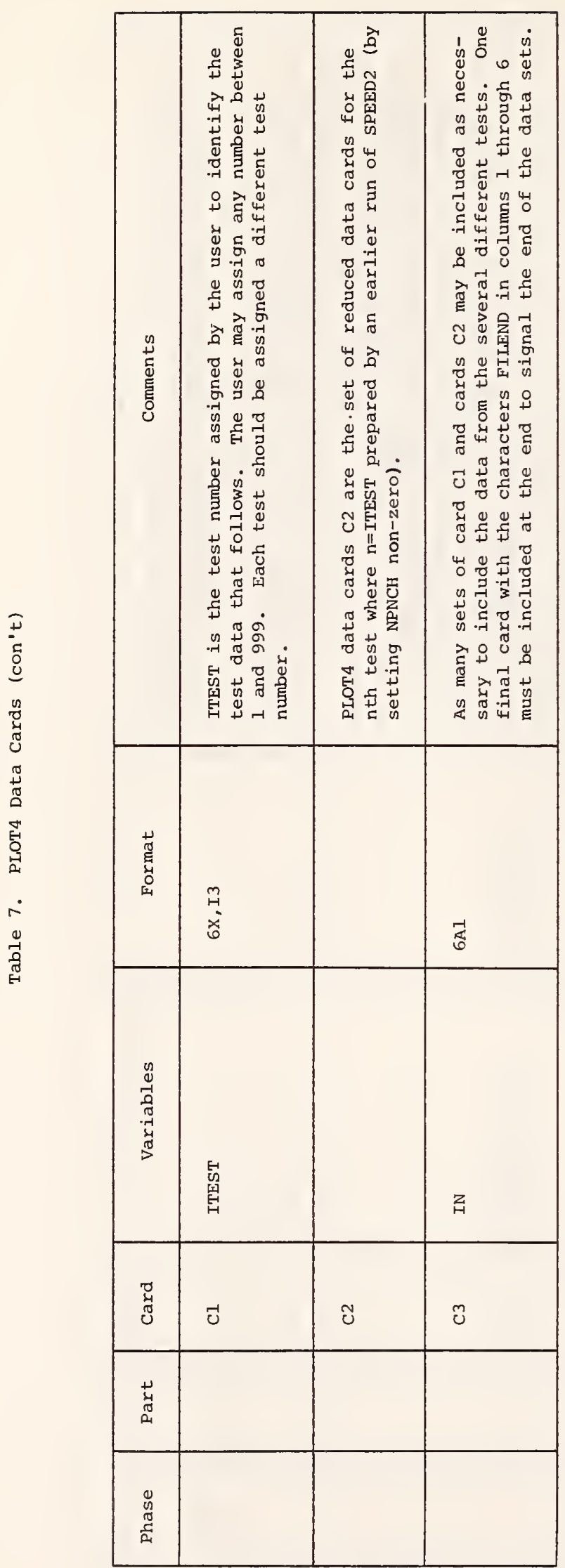


APPENDIX A

PROGRAM STATEMENT LISTING OF SPEED2

A listing of all subprograms of the SPEED2 system follows. A program flow / program interdependency diagram is shown in figure A-1. While the user need not be concerned with most subprograms, all are included for completeness. Subprograms SPEED2 and CONV are the only two that users would normally change. All other changes should only be attempted by those with a detailed knowledge of the SPEED2 system.

\begin{tabular}{|c|c|c|}
\hline Subprogram & Comments & Page \\
\hline SPEED 2 & (UNIVAC 1108 only) & $A-3$ \\
\hline SPEED 2 & (alternate main program) & $A-6$ \\
\hline PLOT1 & & $A-9$ \\
\hline PLOT2 & & $A-10$ \\
\hline PLOT 3 & t. & $A-15$ \\
\hline PLOT4 & & $A-25$ \\
\hline DEF INE & & $A-28$ \\
\hline DATAIN & & $A-39$ \\
\hline DREAD & & $A-43$ \\
\hline MATCH & & A-46 \\
\hline DIGIT & & $A-49$ \\
\hline SKIP & & $\mathrm{A}-50$ \\
\hline REDCIN & & $A-51$ \\
\hline PRINT & & $A-53$ \\
\hline PUNCH & & $A-55$ \\
\hline CONV & & $A-57$ \\
\hline CORR & & $A-58$ \\
\hline FIND & & $A-60$ \\
\hline DRAW & & $A-61$ \\
\hline TEXT & & $A-64$ \\
\hline RWERR & (entry point in subprogram ERROR) & $A-65$ \\
\hline RWEOF & (entry point in subprogram ERROR) & $A-65$ \\
\hline BYTE & (UNIVAC 1108 only) & $A-66$ \\
\hline EREXIT & (UNIVAC 1108 only) & $A-67$ \\
\hline MAP & (UNIVAC 1108 on $1 \mathrm{y}$ ) & $A-68$ \\
\hline
\end{tabular}




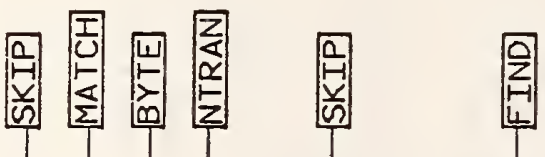

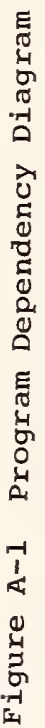

崔
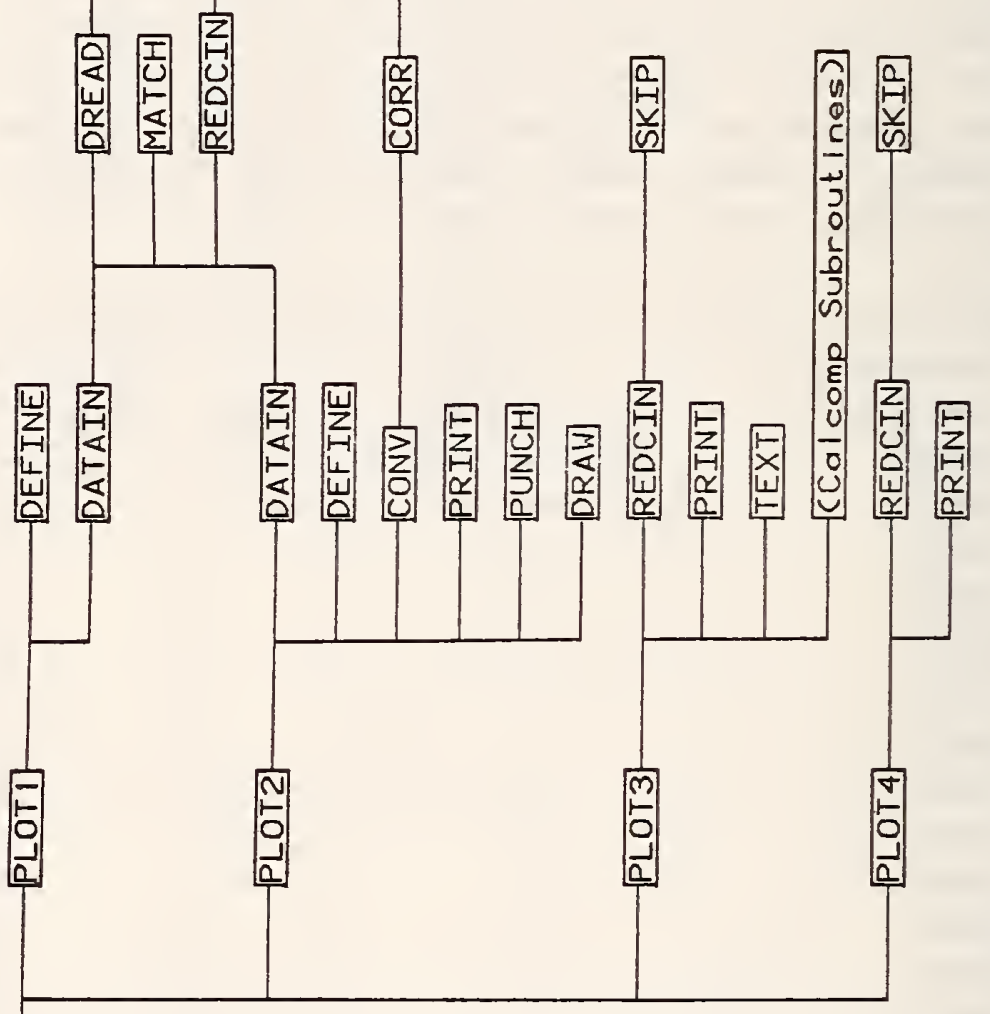

佥

|-

$\ddot{j}$ 


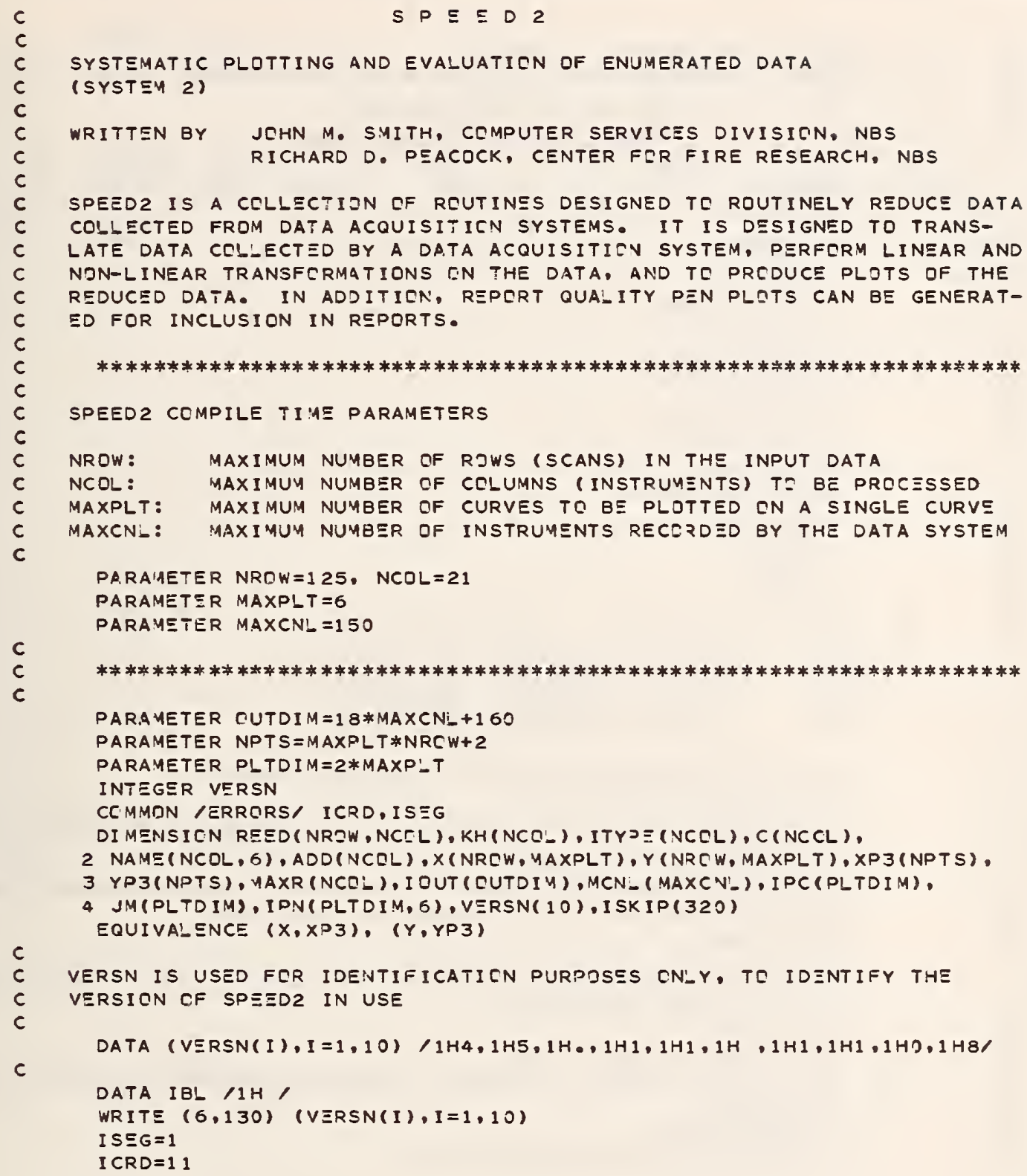




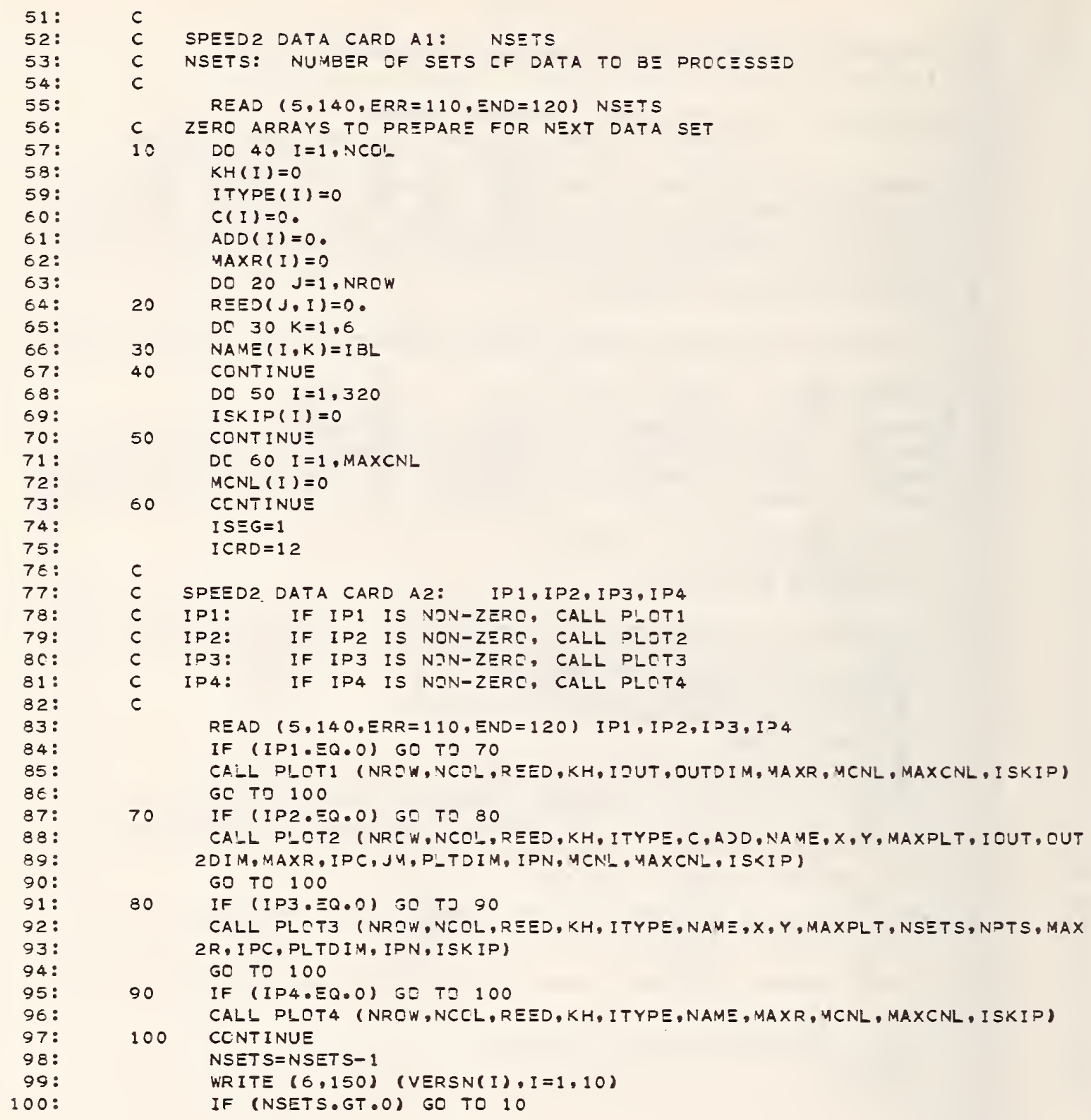




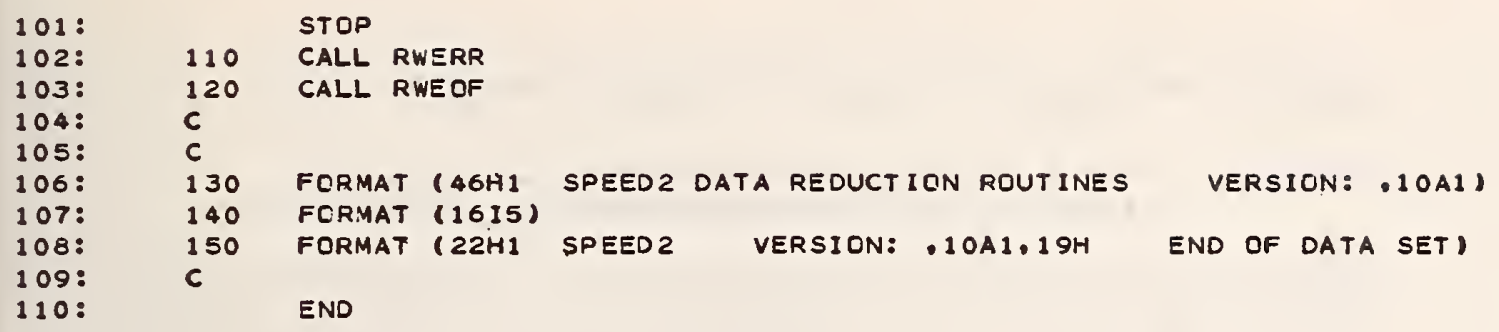

FORMAT ( 46 HI SPEED2 DATA REDUCTION ROUTINES FCRMAT (1615) FORMAT (22H1

SPEED 2

VERSION: $10 A 1,19 H$

END OF DATA SET,

VERSION: .1OA1J

140

150

c

END 


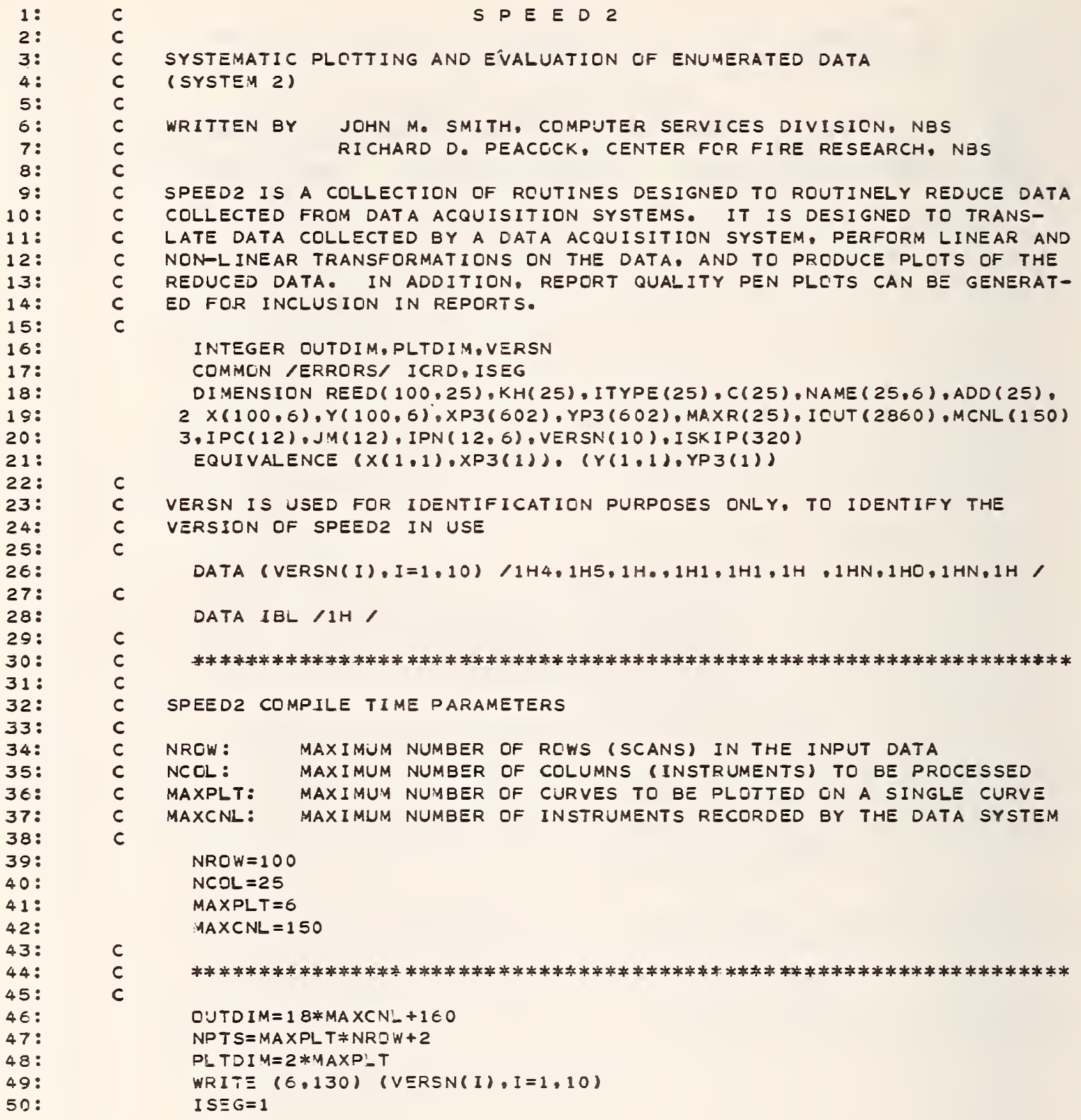


$51:$

52:

53:

54:

55:

$56:$

$57:$

58:

$59:$

60:

$\epsilon 1:$

62:

63:

64:

65:

66:

67:

68:

69:

70 :

$71:$

$72:$

$73:$

$74:$

$75:$

76:

$77:$

73:

$79:$

80 :

81:

82:

83:

84:

85 :

$86:$

$87:$

88:

89:

90:

91 :

92 :

$93:$

94:

95 :

$96:$

$97:$

98 :

$99:$

$100:$

$I C R D=11$

SPEED2 DATA CARD A1: NSETS

NSETS: NUMBER OF SETS CF DATA TO BE PRQCESSED

READ $(5,140, E R R=110, E N D=120)$ NSETS

C ZERC ARRAYS TO PREPARE FOR NEXT DATA SET

10 DO $40 \quad I=1, N C O L$

$K H(I)=0$

$I T Y P E(I)=0$

$C(I)=0$.

$\operatorname{ADD}(I)=0$.

$\operatorname{MAXR}(I)=0$

DC $20 J=1, N R C W$

20 REED $(J, I)=0$.

DC $30 \quad K=1,6$

NAME $(I, K)=I B L$

30

40

CONTINUE

DO $50 \quad I=1,320$

$I S K I P(I)=0$

50 CONTINUE

$D=60 I=1$, MAXCNL

MCNL $(I)=0$

60 CONTINUE

I $S E G=1$

I $C R D=12$

c

c

c

c

c

c

C

SPEED2 DATA CARD A2: IP1,IP2,IP3,IP4

IP 1: IF IPI IS NON-ZERO, CALL PLOT1

IP2: IF IP2 IS NJN-ZERC, CALL PLQT2

IP3: IF IP3 IS NJN=ZERO, CALL PLOT3

IP4: IF IP4 IS NEN-ZERC, CALL PLOT4

READ $(5,140, E R R=110, E N D=120)$ IP $1, I P 2, I P 3, I P 4$

IF (IPI.EQ.0) GC TO 70

CALL PLDTI (NROW,NCOL, REED,KH, IOUT, OUTDIM, MAXR, MCNL, MAXCNL, ISKIP) GD TO 100

70 IF (IP2.EQ.0) GO TO 80

CAL' PLOT2 (NROW, NCOL, REED, KH, ITYPE, C, A DD,NAME, X, Y, MAXPLT, I OUT, OUT 2DIM, MAXR, IPC, JY, PLTDIM, IPN, MCNL, MAXCNL, I SKIPI

GC TO 100

80 IF (IP3, $\equiv \mathrm{Q} .0)$ GC TD 90

CALL PLOT3 (NRCW, NCCL, REED, KH, ITYPE, NAME, X, Y, MAXPLT, NSETS, NPTS, MAX 2R, IPC,PLTDIM, IPN, ISKIP)

GC TO 100

90

IF (IP4.EQ.0) GO TC 100

CALL PLOT4 (NROW, NCOL,REED, KH, ITYPE, NAYE, MAXR, MCNL, MAXCNL, ISKIP)

100 CONTINUE

NSETS $=$ NSET $S-1$

WRITE $(6,150) \quad(V E R S N(I), I=1,10)$ 
$101:$

102:

103:

104:

105:

106:

107:

108:

1C9:

110:

111:

IF (NSETS.GT.O) GO TO 10

STOP

110 CALL RWERR

120 CALL RWEOF

c

c

130 FORMAT (46H1 SPEED2 DATA REDUCTION ROUTINES VERSION:, 1วA1)

140 FCRMAT (16I5)

150

c

FCRMAT ( $22 \mathrm{H}$ I SPEED?

VERSICN: , IOAI, 19H

END GF DATA SET)

END 
1:

2:

3:

4:

5:

6:

7:

8:

9:

10 :

11:

12:

13:

14:

$15:$

$16:$

$17:$

$18:$

19:

20:

21:

22:

23:

24:

25:

26:

27:

28:

29:

30:

31:

32:

33:

34:

$35:$

$36:$

$37:$

38:

39:

40:

41:

42:

43:

44:

45:

46:

$47:$

48:

49:

SUBROUTINE PLOTI (NROW, NCCL,REED,KH, IOUT, IUTDIM, MAXR, MCNL, MAXCNL, 2 ISKIP)

c

C

C

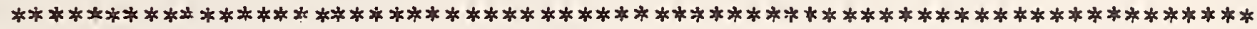

COMMON IERRCRS/ ICRD, ISEG

INTEGER OUTDIM

DI MENSION REED(NRDW, NCQL), KH(NCQL), IOUT (CUTDIM), TITLE (120),

$2 \operatorname{MAXR}(N C C L)$, INDAS(22), ICHRS(320), MCNL (MAXCNL), I SKIP(320)

I SE G $=2$

$I C R D=11$

C

PLOT 1 DATA CARD AI: INTYPE, INPRT, INPNCH, INERR, INSKIP

INTYPE: UNCCNVERTED INPUT DATA FILE TYPE

INPRT: DIRECTS PRINTING CF UNCENVERTED INPUT DATA

INPNCH: DIRECTS PUNCHING CF UNCCNVERTED INPUT DATA

INERR: NUMBER OF INPUT ERRER MESSAGES TO BE PRINTED

INSKIP: DIRECTS SKIPPING OF INPUT RECQRDS

READ $(5,30, E R R=10, E N D=20)$ INTYPE, INPRT, INPNCH, INERR, INSKIP

$I C R D=21$

PLOT1 DATA CARDS B1EB2: TITLE

TITLE: SPECIFIES TITLE OF EXPERIMENT PRINTED AT TOP CF EACH PAGE

$R \subseteq A D(5,40, E R R=10, E N D=20) \quad(T I T L E(I), I=1,120)$

WRITE $(6,50)$

WR ITE $(6,60)$ INTYPE, INPRT, INPNCH, INERR, INSK IP

NR $=$ NRC $W * N C C^{\prime}$

$\mathrm{NC}=1$

$\mathrm{KH}(1)=-1$

ITI MS=1

CALL DEF INE (INTYPE, ICHRS, INDAS, IREAD, I T IM, I EOR, I EOF, MEDIA, TITLE, I 2 NSKIP, ISKIP, NCNL, NTFCRMI

CAL- DATAIN (NR, NC, REED, KH, INTYPE, INPRT, INPNCH, MAXR, ITIME, INERR, IN 2SKIP, IOUT, CUTDIM, I CHRS, INDAS, IREAD, ITIM, IEOR, IECF, MEDIA, TITLE, MCNL 3, MAXCNL, ISKIP, NCNL, NTFCRM)

RETURN

10 CALL RWERR

20 CALL RWEOF

C

C

3) FORMAT (16I5)

40 FORMAT (80A1/40A1)

50 FCIRMAT (27HO PLCTI CCNTRCL PARAMETERS, /)

60

FCRMAT ( $8 H$ INTYPE:, I 5,2X,7H INPRT:, I5,2X,7HINPNCH:, I5,2X,7H INERR: 2. I5.2X,7HINSKIP:, I5)

END 
$1:$

2:

3:

4:

5:

6:

$7:$

8:

9 :

$10:$

11 :

12:

$13:$

14 :

$15:$

16 :

$17:$

$18:$

19 :

20:

21:

$22:$

$23:$

24:

25 :

2E:

27:

28 :

29:

30:

31 :

$32:$

33:

34:

35:

36 :

37 :

$38:$

39:

40:

41:

42:

43 :

44:

45 :

46 :

47:

48 :

$49:$

50 :

SUBROUTINE PLET2 (NROW,NCOL,REED,KH,ITYPE, C, ADD,NAME, X, Y, MAXPLT, 2 I IUT, OUTDIM, MAXR, IPC, JM, PLTDIM, IPN, MCNL, MAXCNL, ISKIP)

COMMCN /EFRORS/ ICRD, ISEG

INTEGER QUTOIM, PI-TDIM, CHAR

DI MENSICN REED(NRCW, NCOL), KH(NCOL), ITYPE (NECL),C(NCCL), ADO(NCOL), 2 NAME(NCOL,5),X(NRCW,MAXPLT),Y(NRCW,MAXPLT), MAXR (NROW).

3 IOUT(CUTDIM), TITLE (120), IN 80$), C H A R(10), I C(6), I P C(P L T) I M)$,

$4 \mathrm{JM}(P L T D I M)$, IHEAD (BO), I SYM(6), ICHRS (320), IVDAS(22), IPN(PLTDIM, 6),

5 I BUFF (40), JBUFF ( 40$), M C N L(M A X C N$, ), ISKIP ( 320$)$

DATA (CHAR (J), J=1,10) / $1 \mathrm{HO}, 1 \mathrm{H1}, 1 \mathrm{H} 2,1 \mathrm{H3}, 1 \mathrm{H4}, 1 \mathrm{H} 5,1 \mathrm{H} 6,1 \mathrm{H7}, 1 \mathrm{HB}, 1 \mathrm{H9/}$

DATA (ISYM(J),J=1,6) / IH*, IHX, IHO, 1H+, 1H-, IH,/

DATA NSEMI, NCEY /1H:1H,

ISEG $=3$

$I C R D=11$

PLCT2 DATA CARD A1: INTYPE, INPRT, INPNCH, INSTCP, INERR, INSKIP INTYPE: UNCQNVERTED INPUT DATA FILE TYPE

INPRT: DIRECTS PRINTING DF UNCQNVERTED INPUT DATA

INPNCH: DIRECTS PUNCHING CF UNCQNVERTED INPUT DATA.

INSTCP: IF INSTCP IS NON=ZERO, STOP PRCCESSING

INERR: NUMBER CF INPUT ERROR MESSAGES TO BE PRINTED

INSKIP: DIRECTS SKIPPING CF INPUT RECQRDS

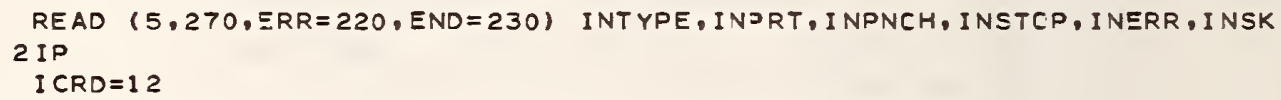

READ $(5,270, E R R=220, E N D=230)$ INTYPE, INJRT, INPNCH, INSTCP, INERR, INSK 2 IP

$I C R D=12$

PLOT2 DATA CARD A2: NTEST,NPRT, NPNCH, NPLOT, NCORR

NTEST: TEST NUMBER

NPRT: DIRECTS DRINTING CF CONVERTED DATA

NPNCH: DIRECTS PUNCHING CF CONVERTED DATA

NPLOT: IF NPLOT IS NJN-ZERD. PRINTER PLJTS ARE TC BE GENERATED

NCORR: IF NCORR IS NON-ZERC, READ CORRECTICNS TO DATA

READ $(5,270, E R R=220, E N D=230) \quad N T E S T, N P R T, N P N C H, N P L C T, N C D R R$

I $C R D=21$

C

PLOT2 DATA CARDS $31 E B 2:$ TIT!E

TITLE: SPECIFIES TITLE OF EXPERIMENT PRINTED AT TCP CF EACH PAGE

READ $(5,280, E R R=220, E N D=230)(T I T-E(I), I=1,120)$

WRITE $(6,290)$

WRITE $(6,390)$ INTYPE, INPRT, INPNCH, INSTIP, I VERR, INSK IP

WRI TE $(6,400)$ NTEST, NPRT, NPNCH, NP'-OT, NCERR

$K=1$

IT I $M E=0$

WRITE $(6,300)$ 


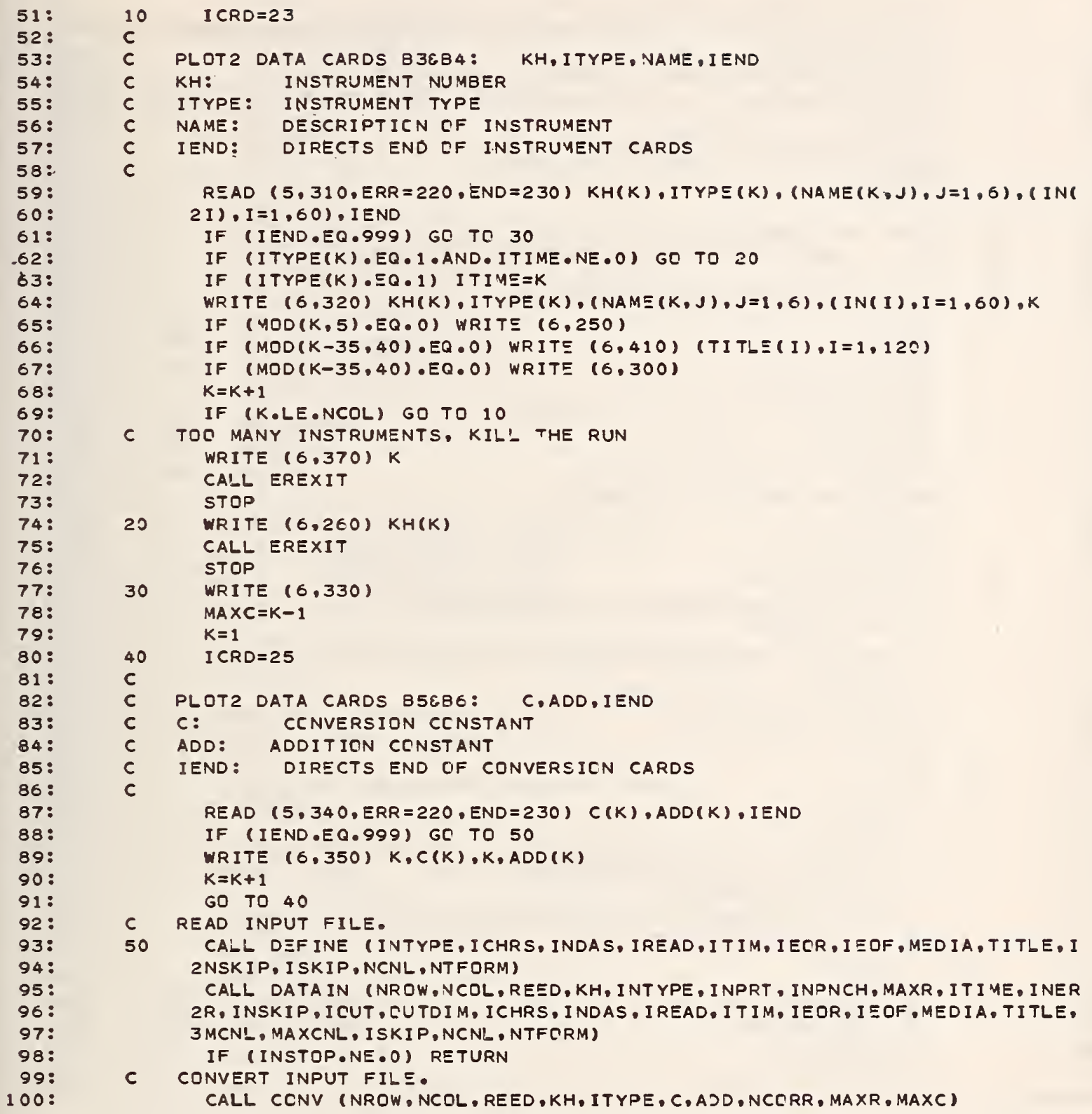




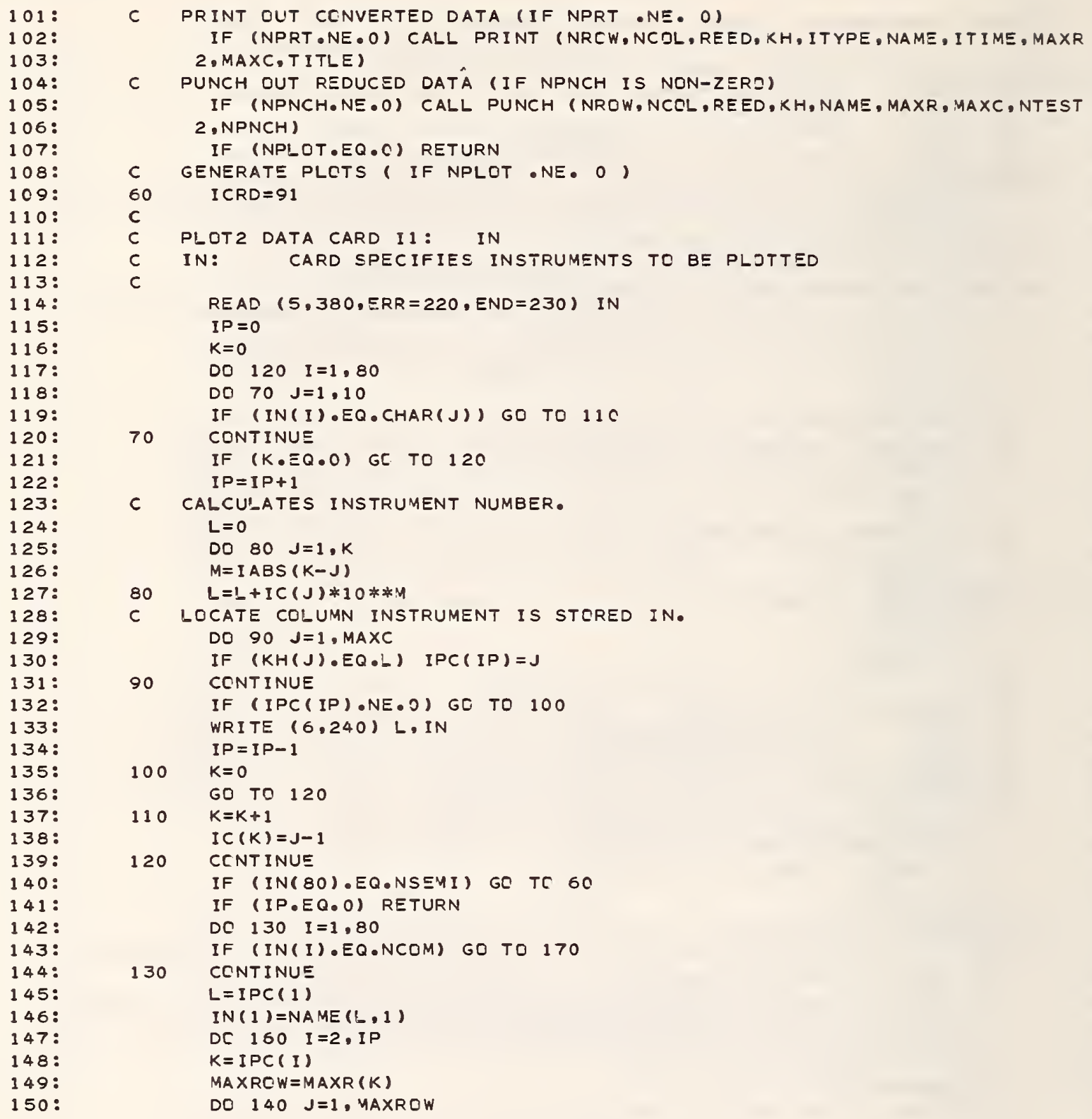




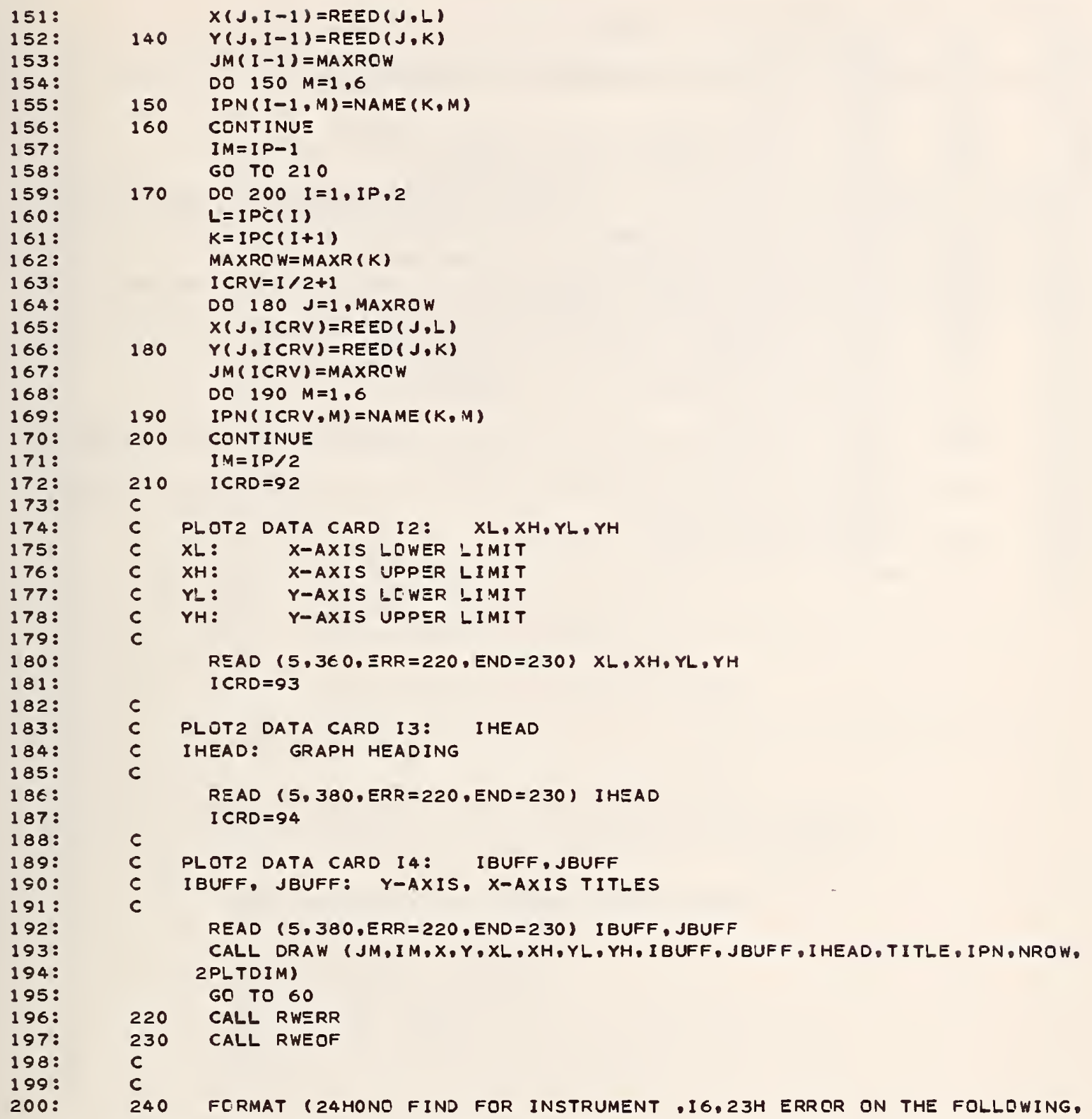


$201:$

202:

203:

204:

205:

206:

$207:$

208:

209:

210:

211:

212:

$213:$

214:

$215:$

$216:$

217 :

218 :

$219:$

220:

221:

222:

223:

224:

225:

226:
210 PLOT CARD, /, 1X,80A1)

FORMAT ( $\left.1 \mathrm{H}^{\circ}\right)$

FORMAT ( 41 HOCN.Y ONE INSTRUMENT OF TYPE 1 IS ALLOWED,

$235 \mathrm{H}$ SECOND FCUND AT INSTRUMENT NUMBER, 13)

FORMAT (16I5)

FORMAT (80A1/40A1)

FORMAT (27HO PLOT2 CCNTRCL PARAMETERS/)

FCRMAT (22HO TABLE OF INSTRUMENTS/,6X,

$241 \mathrm{HKH}$ ITYPE I.D. INSTRUMENT DESCRIPTICN,T87,6HCCLUMN,/,1H,

$392(1 \mathrm{H}-), /)$

FCRMAT (16,I2,66A1,3X, I3)

FORMAT ( $1 X, I 6, I 3,3 X, 6 A 1,2 X, 60 A 1, T 87, I 6)$

FORMAT (//,22HOTABLE OF MULTIPLIERS, $10 X, 15 H T A B L E$ OF ADDERS)

FORMAT $(1 X, F 15,6, F 15,6,149)$

FCRMAT (2H C,I2, IH=,F $15,6,12 X, 3 H A D D, I 2,1 H=, F 15.6)$

FORMAT (4F10.0)

FORMAT ( 42 HOCCLUMN STORAGE LIMITS EXCEEDED AT CCLUMN, I4,/,

$231 \mathrm{H}$ CHECK PARAMETERS NCCL AND NROW) FORMAT (BOA1)

380

390

FORMAT ( $8 H$ INTYPE:, I5,2X,7H INPRT:, I5,2X,7HINPNCH:, I5,2X,7HINSTOP:

2. I 5, 2X,7H INERR:, I5, 2X, 7HINSK IP:, I5)

400 FORMAT (8H NTEST:, I5,2X,7H NPRT:, I5,2X,7H NPNCH:, I5,2X,7H NPLOT: 2, I5,2X,7H NCORR:, I5)

410 FCRMAT (1H1,120A1)

C

END 
1:

2:

3:

4:

$5:$

6:

7:

8:

9:

10:

11 :

12:

13:

14:

15:

16:

$17:$

18:

19:

20:

21:

22:

23:

24:

25:

26:

27:

28:

29:

30:

31:

32:

$33:$

34 :

35:

36:

$37:$

38 :

39:

40:

41:

42:

43:

44:

45:

46:

47:

48:

49:

50:

SUBROUT INE PLCT3 (NROW, NCCL, REED, KH, ITYPE, NAME, X, Y, MAXPLT, NSETS, 2 NPTS, MAXR, IPC, PLTDIM, IPN, ISKIP)

C

C

CQMMON /ERRORS/ICRD,ISEG

INTEGER CHAR,PLTDIM, XPOW,XDIG,YPOW, YDIG, FTIT

REAL IY, IX

DIMENSION REED(NROW, NCOL), KH(NCOL), ITYPE (NCOL), NAME (NCOL, 6$)$, 2. TITLE(120), IN(80), CHAR(10), IC(6), IPC(PLTDIM), AREA(1024), IBUFF(40) 3. JBUFF $(40), X(N P T S), Y(N P T S)$, I HEAD $(78), M A X R(N C O L)$, IPN (PI-TDIM, 6$)$, 4 FTIT (10), ISKIP(320)

DATA (CHAR(J),J=1,10)/1HO,1H1,1H2,1H3,1H4,1H5,1H6,1H7,1H8,1H9/

DATA (FTIT (J),J=1,10)/1HF, 1HI,1HG, $1 \mathrm{HU}, 1 \mathrm{HR}, 1 \mathrm{HE}, 1 \mathrm{H}, 1 \mathrm{H}, 1 \mathrm{H}, 1 \mathrm{H}: /$

DATA NCOM,NSEMI,NS, NP,NL /1H, $1 \mathrm{H} ;, 1 \mathrm{HS}, 1 \mathrm{HP}, 1 \mathrm{HL}$

C

INITIALIZE THE CALCOMP TAPE FOR THE PLOTTING

CALL PLOTS (AREA,1024,7)

CALL PLOT $(0, \ldots-11, \ldots-3)$

CALL PLOT $(0,1,5,-3)$

WRITE $(6,710)$

C

I $S E G=4$

$I C R D=11$

C

PLOT3 DATA CARD A1: NPRT, NSMTH, NOUT, NAXIS,NPNT, NLEG

NPRT: IF NPRT IS NON-ZERO, PRINT DATA

NSMTH: SMCCTHING FACTOR

NCUT: DETERMINES FATE OF CUT-OF-BOUNDS PCINTS

NAXIS: TWO OR FQUR SIDED AXES

NPNT: $\quad$ SPACING DF POINTS ON CURVES

N'EG: READ CARDS FIR LEGENDS CN CURVES

READ $(5,720, E R R=630, E N D=640) \quad$ NPRT, NSMTH, NOUT, NAXIS, NPNT, NLEG

$I C R D=12$

C

C

PLOT 3 DATA CARD A2: XSIZE, YSIZE, SCALE

XSIZE: LENGTH CF THE $X$ AXIS OF THE PLOT IN INCHES

YSIZE: LENGTH CF THE Y AXIS OF THE PLOT IN INCHES

SCALE: SCALE FACTOR FCR PLOTTING

READ $(5,880, E R R=630, E N D=640) \quad X S I Z E, Y S I Z 5, S C A L E$

IF (XSIZE.LT.1.J) XSIZE $=10.0$

IF (YSIZE.LT.1.0) YSIZE $=8.0$

IF (SCALE.EQ.0.) SCALE $=1$. ?

IF (NSMTH.LT.O) NSMTH=0

CALL FACTOR (SCALE)

WRITE $(6,730)$ NPRT, NSMTH, NOUT, NAXIS, NPNT, NLEG

WRITE $(6,740)$ XSIZE, YSIZE, SCALE

I CRD $=21$

C 


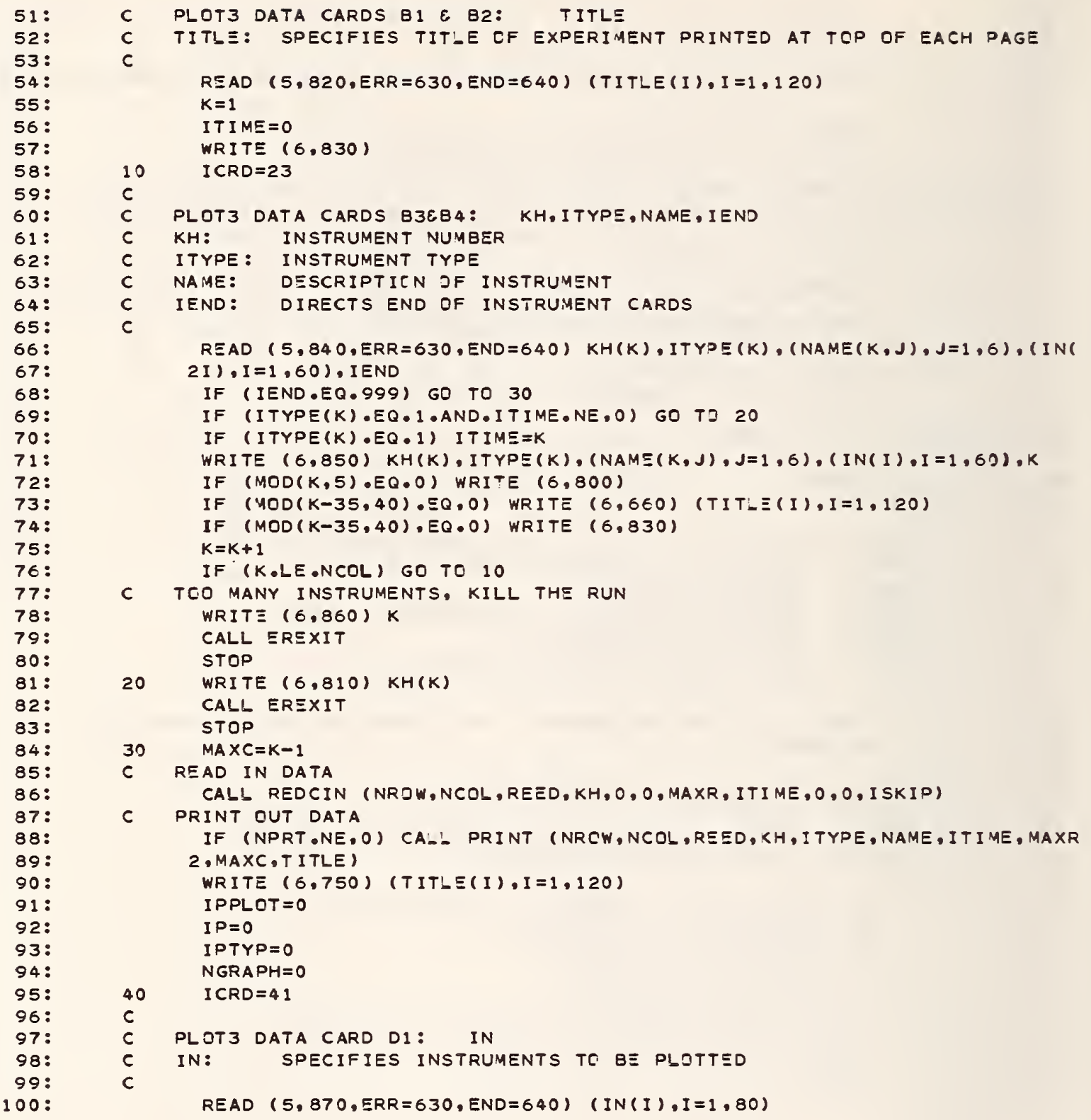




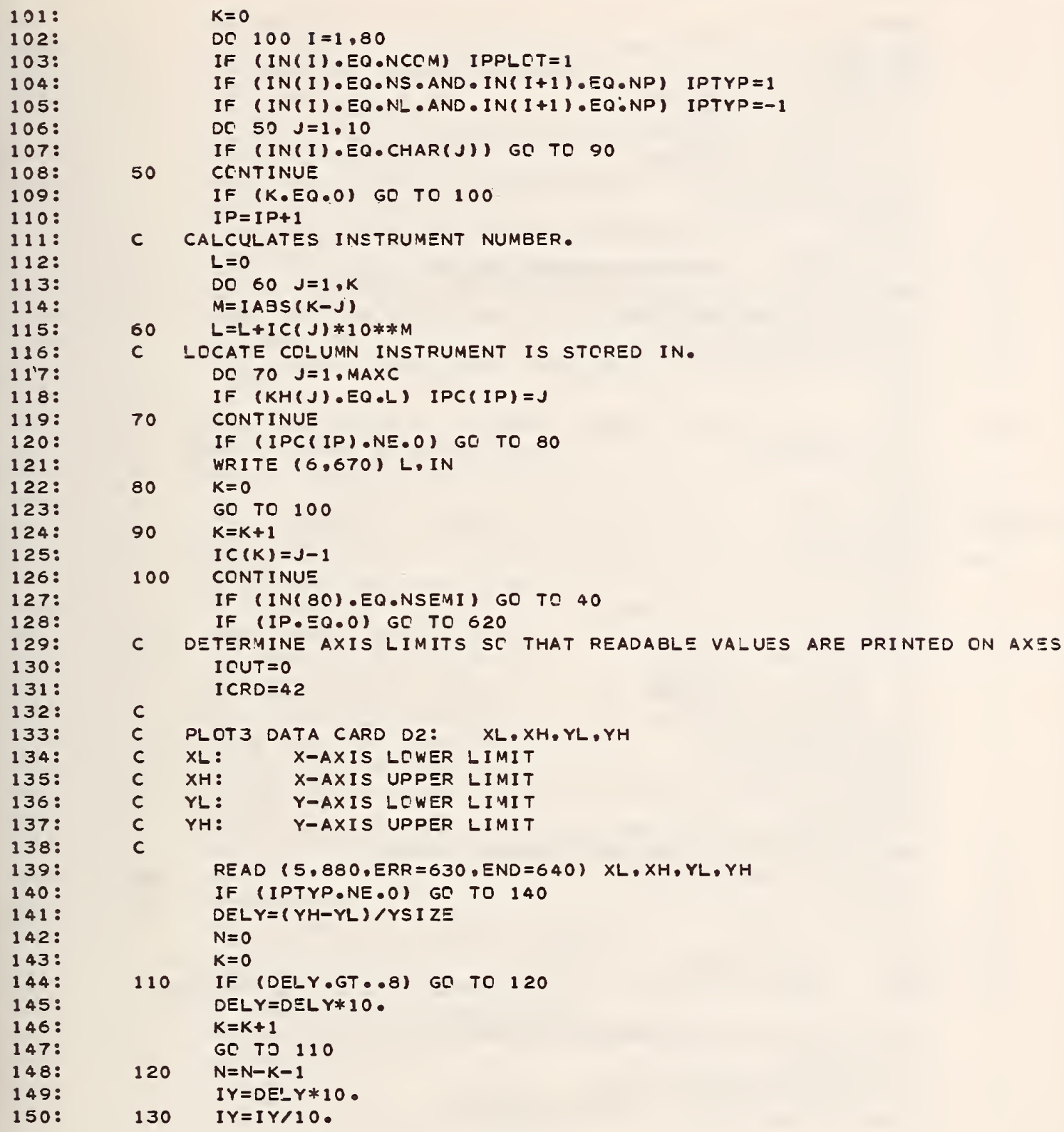




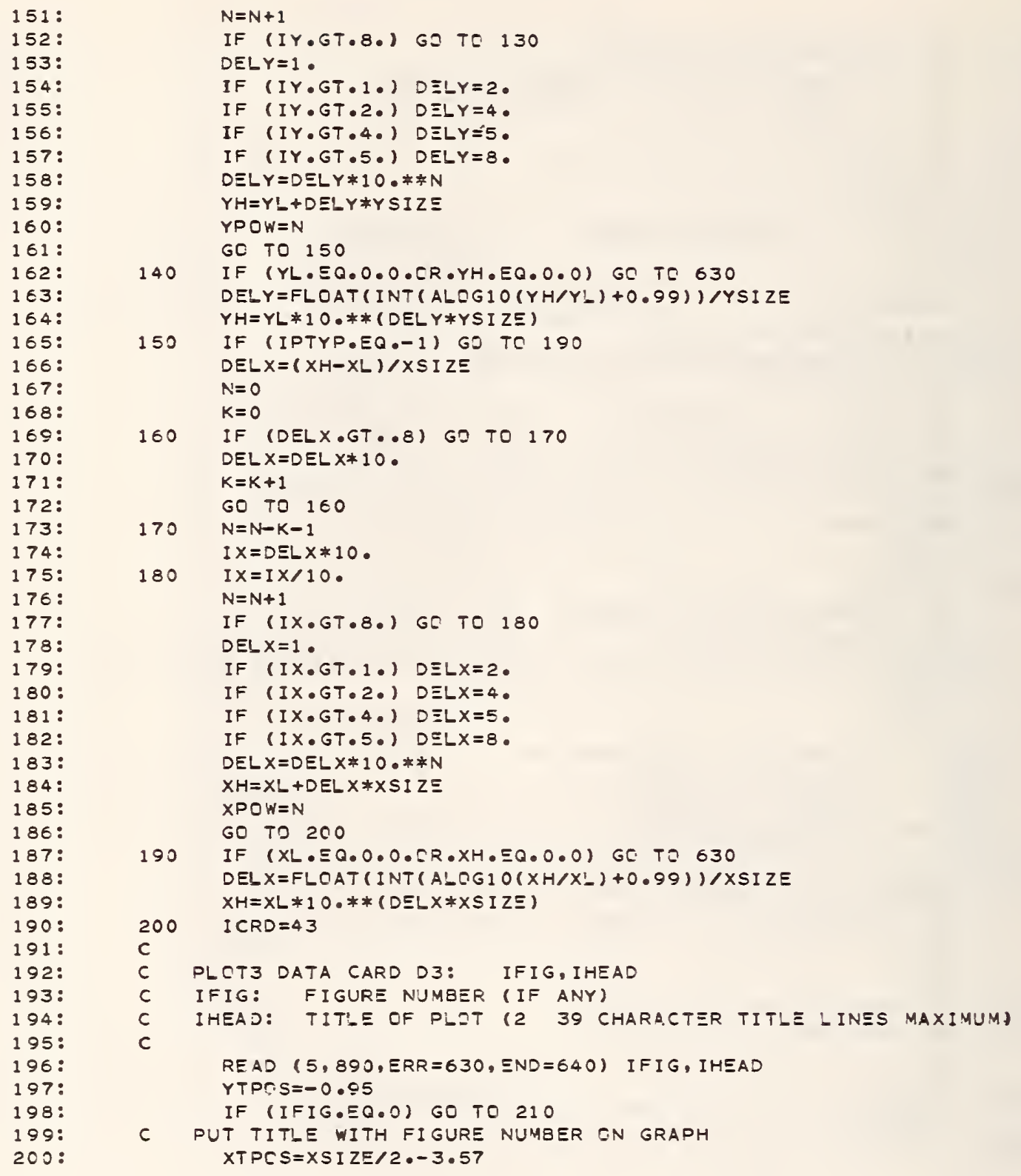




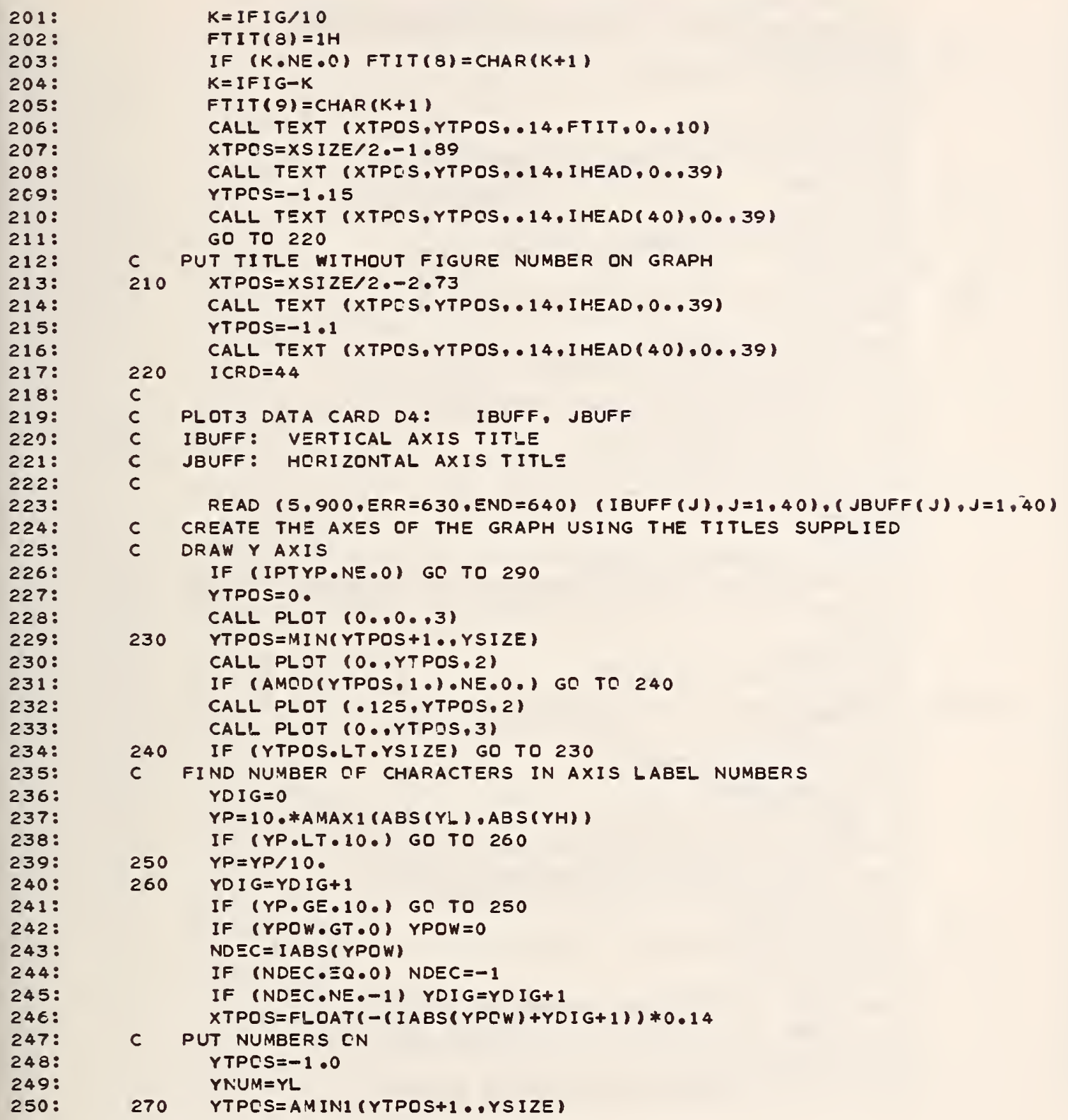


251:

252:

253:

254:

255:

256:

257:

258:

259:

260 :

261:

262:

263:

264:

$265:$

266:

267:

268:

$269:$

$270:$

271:

272:

273:

274:

275:

276:

277:

278:

$279:$

280:

281 :

282:

283:

284:

285:

286:

$287:$

288:

289:

290:

291:

292:

293:

294:

295:

296:

297:

298:

299:

उ00:
IF (AMCD(YTPOS, 1.).NE.O...ND.YTPOS.EQ.YSIZE) GO TO 280 CALL NU:ABER (XTFOS, YTPCS-0.07,.14,YNUM, O. NDEC)

280 YNUM=YNUM+DELY

C AXIS TITLE

IF (YTPES.LT.YSIZE) GE TO 270

$$
\text { YTPOS }=Y S I Z E / 2 \cdot-2 \cdot 8
$$

$X T P O S=X T P C S-.20$

CAL' TEXT (XTPOS,YTPOS,.14, IBUFF,90, 40)

GO TO 300

290 CALL LGAXIS $(0,0,0,1 \mathrm{H}, 0, Y S I Z E, 90,, \mathrm{Y}$, DELY)

YTPOS $=Y S I Z E / 2,-2 \cdot 8$

CALL TEXT $(-0.5$, YTPOS,.14, IBUFF,90.,40)

$C$ DRAW $X$ AXIS

300 IF (IPTYP.5Q.-1) GO TC 370

$X T P O S=0$.

CALL PLOT $(0,10,3)$

310 XTPOS $=$ A YIN1 $(X T P O S+1 \ldots X S I Z E)$

CALL PLOT (XTPOS,0.,2)

IF (AMOD(XTPCS, 1.).NE.0.) GO TC 320

CALL PLJT (XTPOS,.125.2)

CALL PLOT (XTPCS,0,3)

320 IF (XTPOS.LT.XSIZE) GO TO 310

$C$ PUT NUMBERS BELCW TIC MARKS $C N \times$ AXIS $X T P O S=-1$. $X N \cup M=X l$

330 XTPGS=AMINI (XTPOS+1 .XSIZE)

IF (AMOD(XTPCS, 1.).NE.O..AND.XTPQS.EQ.XSIZE) GO TO 360

C FIND NUMBER OF CHARACTERS IN AXIS LABEL NUMBER $X D I G=0$

$X P=10 . * X N U M$

IF $(X P \cdot L T \cdot 10$,$) GO TO 350$

$340 \quad X P=X P / 10$.

$350 \quad X D I G=X D I G+1$

IF $(X P, G E \cdot 10$,$) GE TD 340$

IF $(X P O W \cdot G T \cdot O) \quad X P O W=0$

$N D E C=I A B S(X P O W)$

IF (NDEC.EQ.O) NDEC $=-1$

IF (NDEC.EQ.-1) XDIG=XOIG-1

$X T P O S=X T P O S-F L D A T((I A B S(X P C W)+X D I G+1)) * 0.07$

CALL NUMBER (XTPOS, -.19,.14,XNUM, 0.,NDEC)

$X T P C S=X T P O S+F L D A T((I A B S(X P O W)+X D I G+1)) * 0.07$

$360 \quad X N U M=X N U M+D E L X$

IF (XTPOS.LT.XSIZE) GO TC 330

C ADD AXIS TITLE

$X T P O S=X S I Z E / 2 \cdot-2.8$

CALL TEXT (XTPOS, -.53,.14, JBUFF, 0.,40)

GC TJ 380

370 CALL LGAXIS $(0,0,0,1 \mathrm{H},-1, X S I Z E, 0, \ldots$ XL, DELX)

$X T P C S=X S I Z E / 2 \cdot-2 \cdot 8$

CALL TEXT (XTPJS, $-0.53, .14, J B U F F, 0.40)$ 
$301:$

302 :

303:

304:

305 :

306 :

307 :

308:

$309:$

310 :

311:

312 :

313 :

314:

315 :

316 :

317 :

318 :

$319:$

320:

321:

322:

323:

324:

325:

326 :

327:

328:

329:

330 :

331:

332:

333 :

334:

335:

336:

$337:$

338 :

339:

340:

$341:$

342 :

343 :

344 :

$345:$

346 :

$347:$

348 :

349:

$350:$

380 IF (NAXIS.EQ,C) GO TO 430

C PUT AXES ON THE OTHER TWO SIDES OF THE GRAPH, Y AXIS FIRST YTPOS $=0$.

CALL PLCT (XSIZE,0,3)

390 YTPC.S=AMINI ( YTPOS+1 , YSIZE)

CALL PLOT (XSIZE,YTPQS, 2)

IF (AMOD(YTPCS,1.).NE.0.) GC TC 400

CALL PLOT (XSIZE-.125,YTPOS, 2)

CALL PLOT (XSIZE,YTPOS, 3)

400 IF (YTPOS.LT.YSIZE) GO TO 390

$C$ DRAW $X$ AXIS

$X T P O S=0$.

CALL PLCT $(0,, Y S I Z E, 3)$

410 XTPOS=A.MINI (XTPIS+1, XSIZE)

CALL PLCT (XTPOS,YSIZE, 2)

IF (AMCD(XTPCS, 1.). NE.0.) GE TO 420

CALL PLOT (XTPOS,YSIZE- 125,2 )

CALL PLOT (XTPCS,YSIZE,3)

420 IF (XTPOS.LT.XSIZE) GO TC 410

$430 \quad$ NCRV $=I P-1$

IF (IPPLOT •EQ.1) NCRV=IP/2

$D C 540 \quad I=1, N C R V$

IF (IPPLOT.EQ.1) GO TO 440

$I X X=I P C(1)$

$I Y Y=I P C(I+1)$

GC TO 450

$440 \quad I X X=I P C(2 * I-1)$

$I Y Y=I P C(2 * I)$

$450 \quad$ NPT $=0$

MAXRCW $=$ MAXR (I YY)

DO $470 \quad L=1$, MAXRCW

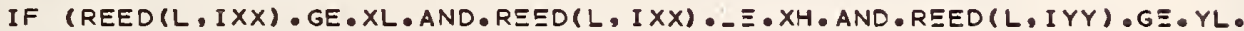

2AND.REED(L, IYY) - $-E, Y H)$ GC TO 460

IOUT $=$ I OUT +1

IF (NOUT,EQ.O) GC TO 470

$460 \quad$ NPT $=$ NPT +1

$X(N P T)=R E E D(L, I X X)$

$Y(N P T)=R E E D(L, I Y Y)$

470 CENTINUE

I SYM $=I-1$

$X(N P T+1)=X L$

$X(N P T+2)=D S L X$

$Y(N P T+1)=Y L$

$Y(N P T+2)=D E L Y$

IF (NPT.EQ.O) GO TC 520

IF (IPTYP.EQ.0) GO TO 480

CAL: LG'-INE (X,Y,NPT, 1, NPNT, I SYИ, IPTYP)

GC TD 520

C SMOCTH DATA IF NSMTH IS NOT EQUAL TC ZERE

480 IF (NSMTH.EQ.O) GO TO $51 \mathrm{C}$ 
351:

352:

353:

354:

355:

356:

357:

358:

359:

360:

361 :

362:

363:

364:

365 :

366:

367:

368 :

369:

370:

371:

372:

373:

374:

375:

376:

377:

378:

379:

380 :

381:

382:

383:

384:

385:

386 :

387:

388:

$389:$

390:

391:

392:

$393:$

394:

395:

396:

397:

398:

399:

400:

DO $500 \quad L=1, N P T$

I PTH $=$ NP $T-1+1$

IPTL $=M A X O(N P T-L-N S M T H=1,1)$

$Y(I P T H)=Y(I P T H) / F L O A T((I P T H=I P T L+1))$

IF (IPTH.EQ.1) GO TO 500

IP THMI = IPTH 1

DO 490 LL=IPTL, IPTHM 1

$Y(I P T H)=Y(I P T H)+Y(L L) / F L O A T((I P T H=I P T-+1))$

490

CONTINUE

CONT INUE

CALL LINE ( $X, Y, N P T, 1, N P N T$, ISYM)

DO $530 \mathrm{~J}=1,6$

IPN $(I, J)=N A M E(I Y Y, J)$

CONTINUE

IF (NLEG.EQ.O) GO TO 550

c

C PLOT3 DATA CARD DS: IS, ISYM, XTPOS, YTPOS, IHEAD

C IS: PLOTTING SYMBOL FI-AG

C ISYM: PLOTTING SYMBOL PREFIXED TC LEGEND IF IS IS NON-ZERC

C XTPCS: X POSITION OF LEGEND

C YTPCS: Y POSITION CF LEGEND

C IHEAD: LEGEND

C

READ $(5,650, E N D=630, E R R=640)$ IS, I SYM, XTPOS, YTPOS, (IHEAJ $(I), I=1,60)$ IF (IS.EQ.O.AND.ISYM.EQ.O.AND.XTPOS.EQ.0.0.AND.YTPES.EQ.0.0.AND.IH 2EAD(1).EQ.1H ) GO TO 550

IF (IS.NE.O) CALL SYMBOL (XTPOS-.105,YTPOS+.035,.07,ISYM,0.,-1)

CALL TEXT (XTPOS,YTPOS,.07, IHEAD, 0.,60)

C PUT A LEGEND BLOCK IN BOTTCM RIGHT HAND CERNER

550 CALL FACTOR (1.0)

$X T P O S=X S I Z E * S C A L E+0.5$

YTPOS $=0.1 \neq F L O A T(N C R V / 2+M O D(N C R V, 2))+0.03$

DC. $580 \quad I=1, N C R V, 2$

YTPOS $=Y T P C S-0.10$

CALL SYMBCL (XTPOS, YTPOS+.035,.07, I-1,0.,-1)

DC $560 \mathrm{~J}=1.6$

$560 \quad \operatorname{IN}(J)=\operatorname{IPN}(I, J)$

CALL TEXT (XTPCS+.14,YTPCS,.07, IN,0.,6)

IF (I.EQ.NCRV) GO TO 590

CALL SYMBCL (XTPCS+0.7, YTPOS+.035,.07,I,0.,=1)

DC $570 \quad J=1,6$

$570 \quad I N(J)=I P N(I+1, J)$

CALL TEXT (XTPOS+0.84,YTPOS,.07, IN, 0.,6)

580 CCNTINUE

$590 \quad$ XTPOS $=X S I Z E * S C A L E+0.4$

YTPC S $=0.1$ *F! $-O A T(N C R V / 2+M O D(N C R V, 2))+0.03$

YLEN $=0.1$ *FLOAT $(N C R V / 2+M O D(N C R V, 2))+0.03$

CALL PIOT (XTPCS, YTPOS, 3)

CAL: PLOT (XTPOS+1.425, YTPOS, 2)

CALL PI_CT (XTPOS+1.425, YTPOS-YLEN, 2) 
401:

$402:$

$403:$

404:

405:

$406:$

407:

408:

409:

410 :

$411:$

412 :

$413:$

414:

415:

A16:

417:

418 :

419:

420:

421:

422:

423:

424:

425 :

$426:$

$427:$

428:

429:

430 :

431:

$432:$

433 :

434:

435:

436 :

$437:$

438 :

439:

440:

441 :

442 :

443:

444 :

445 :

446 :

447 :

448:

449:

450:
CALL PLOT (XTPOS, YTPOS-YLEN, 2 )

CALL PLOT (XTPOS,YTPOS, 2)

CALL FACTOR (SCALE)

C WRITE DUT INFORMATION ABQUT THE GRAPH WE JUST FINISHED

$D E 610 \quad I=1$, IP

$J=I P C(I)$

$I P C(I)=K H(J)$

DC $600 \quad K=1.6$

$600 \quad$ IPN $(I, K)=N A M E(J, K)$

610 CONTINUE

IF (IPPLOT, EQ.0) WRITE $(6,760)((\operatorname{IPN}(I, J), J=1,6), I P C(I), I=1, I P)$

IF (IPPLDT:EQ.1) WRITE $(6,680)$ ( (IPN $(I, J), J=1, \epsilon), I P C(I), I=1$, IP)

WRITE $(6,770) \quad X L, D E L X, X H, Y L, D E L Y, Y H$

WRITE $(6,780)$ JBUFF, I BUFF

IF (IFIG.EQ.0) WRITE $(6.790)$ IHEAD

IF (IFIG.NE.O) WRITE $(6,910)$ IFIG.IHEAD

IF (IOUT•NE•O) WRITE $(6,690)$

IF (YSIZE*SCALE+1.5.GT.10.75) WRITE $(6.700)$

XMOVE $=X S I Z E+7.0$

CALL PLCT (XMOVE.0..-3)

I PPLDT $=0$

I $P=0$

IPTYP=O

NGRAPH $=$ NGRAPH+1

IF (MCD(NGRAPH, 5) EQ.0) WRITE (6,750) (TITLE(I),I =1,120)

GO TO 40

620 IF (NSETS.LE.1) CALL PLOT $(0,0 ., 999)$

RETURN

630 CALL RWERR

640 CALL RWEOF

c

c

650 FCRMAT (I1.I2.2F6.0.60A1)

660 FORMAT (1H1.120A1)

670 FCRMAT ( 31 HOND FIND FOR INSTRUMENT NUMBER . I 6.

$214 \mathrm{H}$ ERROR ON THE 19HFOLLOWING PLDT CARD, $1.1 \times, 80 A 1)$

680 FORMAT (17HOCALCQMP PLCT CF, 2(6A1,2H (. I6,4H), .6A1,2H (, I6.

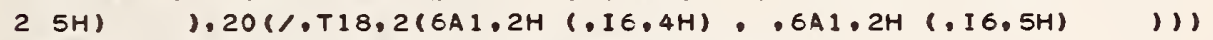

FORMAT (29HOPOINTS OUT OF BOUNDS ON PLJT)

FORMAT ( 48 HOPAPER WIDER THAN 12 INCHES MUST BE USED CN PLJT)

FCRMAT (25HOPLOT 3 CONTROL PARAMETERS)

FCRMAT (16I5)

FCRMAT ( 8 HO NPRT:, I5.2X,7H NSMTH:, I5.2X,7H NOUT:, I5.2X,7H NAXIS:

2.15,2X,7H NPNT:, I5,2X,7H NLEG:.15)

FCRMAT ( $8 H$ XSIZE:,F5.2,2X,7H YSIZE:.F5.2,2X,7H SCALE:,F5.2)

FCRMAT ( 1 H1,120A1,, 23 HOCALCOMP PLOTS PREPARED)

FCRMAT ( 17 HOCALCOMP PLCT CF , GA1,2H $(, I 6,9 H)$ VERSUS,3(2X,6A1,2H (

$2.16,1 H)) .7(/, T 37.3(2 X, 6 A 1,2 H(.16,1 H)))$

FCRMAT ( $21 \mathrm{H}$ X-AXIS LOWER LIMIT: , G8.3.21H

DELX (PER INCH): ,

$268.3 .14 \mathrm{H}$ UPPER LIMIT: . G8.3.\%.21H Y-AXIS LJWER LIMIT: .G8.3. 
451:

452:

453:

454:

455:

$456:$

$457:$

458:

459:

460:

$461:$

462:

463:

464:

465:

466 :

467:

468:

469:

470 :

471:
$321 \mathrm{H}$ DELY (PER INCH): , G8.3,14H UPPER LIMIT:, G8.3)

FCRMAT (15H X-AXIS TITLE: ,40A1./.15H Y-AXIS TITLE: ,40AI)

FORMAT (14H GRAPH TITLE:, 39A1,/,14X,39A1)

FORMAT (1H )

FCRMAT ( 41 HOONLY CNE INSTRUMENT OF TYPE 1 IS ALLOWED,/,

$235 \mathrm{H}$ SECCND FUUND “AT INSTRUMENT NUMBER, I3)

FCRMAT ( $80 A 1 / 40 A 1)$

FORMAT (22HO TABLE OF INSTRUMENTS/,3X,

$241 \mathrm{HKH}$ ITYPE I.D. INSTRUMENT DESCRIPTION,T87,6HCOLUMN, /, IH,

$392(1 \mathrm{H}-1, /)$

FORMAT (I6,I2,66A1,3X,I3)

FORMAT (1X,I6,I3,3X,6A1,2X,60A1,T87,I6)

FCRMAT ( $42 H O C O L U M N$ STCRAGE LIMITS EXCEEDED AT COLUMN, I 4,1,

$231 \mathrm{H}$ CHECK PARAMETERS NCC!- AND NRCW)

FORMAT (BOA1)

FCRMAT (4F10.0)

FCRMAT (12,78A1)

FORMAT (8OA1)

FORMAT (21H GRAPH TITLE: FIGURE, I 2,3H:,39A1, $/, 26 \times, 39 A 1)$

END 
1:

2:

3:

4:

5:

6:

7:

8:

9:

10:

11 :

12:

13:

14:

15:

16:

$17:$

18:

$19:$

20:

21:

22:

23:

24:

25:

26:

27:

28:

29:

30 :

31:

32 :

33:

34:

35:

36 :

37:

38:

39:

40:

41:

42 :

43:

44:

45:

46:

47:

48 :

49:

50 :

SUBROUTINE PLOT4 (NROW, NCOL,REED, KH, ITYPE, NAME, MAXR, MCNL, MAXCNL. 2 ISKIP)

C

$c$

CCMMON /ERRORS/ICRD, ISEG

DI MENSION REED (NRC,W,NCOL), KH(NCOL), ITYPE(NCCL), NAME (NCOL, 6 ),

2 MAXR (NCOL), TITLE(120), IN(80), MCNL (MAXCNL), ISKIP(320)

DATA NF/1HF/

ISEG $=5$

$I C R D=11$

C

C PLOT 4 DATA CARD AI: NPRT,NPNCH

C NPRT: DIRECTS PRINTING EF CCNVERTED DATA

C NPNCH: DIRECTS PUNCHING CF CCNVERTED DATA

c

READ (5,110,ERR $=80, E N D=90) \quad N P R T, N P N C H$

ICRD $=21$

C PLOT4 DATA CARDS BI $\varepsilon$ B2: TITLE

C TITLE: SPECIFIES TITLE OF EXPERIMENT PRINTED AT TCP CF EACH PAGE

C

READ $(5,120, E R R=80, E N D=90) \quad(T I T L E(I), I=1,120)$

WRITE $(6,130)$

WRITE $(6,140)$ NPRT, NPNCH

$K=1$

WRITE $(6.160)$

I CRD $=23$

PLCT4 DATA CARDS B3: ITYPE, KH,NAME, IEND

ITYPE: TEST NUMBER FOR THIS INSTRUMENT

KH: INSTRUMENT (CHANNEL) NUMBER FCR THIS INSTRUMENT

NAME: DESCRIPTIQN OF INSTRUMENT

IEND: DIRECTS END DF INSTRUMENT CARDS

READ (5, 150, ERR $=80, E N D=90)$ I TYPE $(K), K H(K),(N A M E(K, J), J=1,6)$, (IN(I)

$2, I=1,60)$, IEND

IF (IEND•EQ.999) GO TO 20

WRI TE $(6,170) \mathrm{KH}(K)$, ITYPE(K), (NAME(K,J),J=1,6), (IN(J),J=1,60),K

IF $(M O D(K, 5), E Q, 0)$ WRITE $(E, 180)$

IF $(M O D(K-35,40), E Q \bullet 0)$ WRITE $(6,100)$ (TITLE(I),I=1,120)

IF $($ MOD $(K-35,40), E Q .0)$ WRITE $(6,160)$

$K=K+1$

IF (K.LE.NCOL) GO TO 10

C TCC MANY INSTRUMENTS, KILL THE RUN

WRITE $(6,250) \mathrm{K}$

CALL EREXIT

STOP

20 MAXC $=K-1$

30 I $C R D=31$

30 


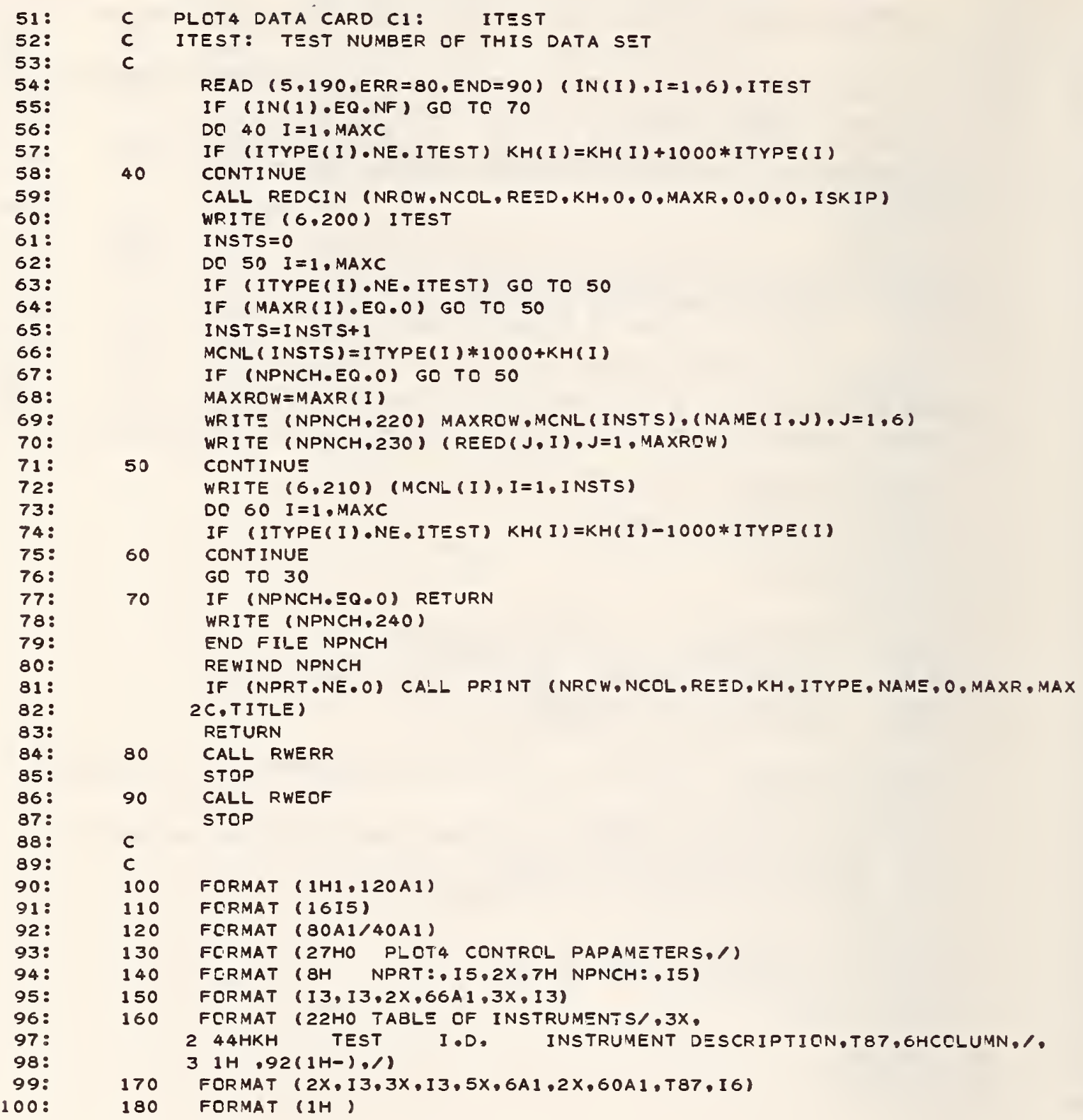




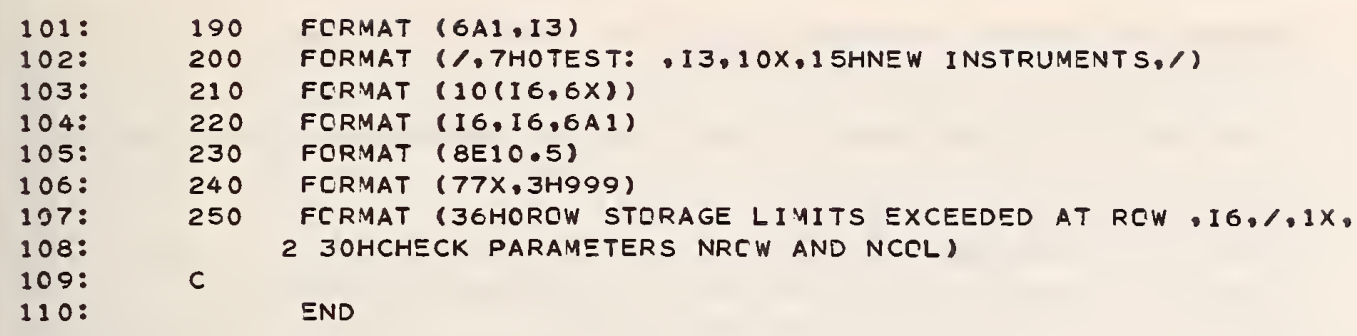


1:

2:

3:

4:

5:

6:

$7:$

8:

9:

10:

11:

12 :

13:

14:

$15:$

16:

17 :

18:

19:

20:

21:

22:

23:

24:

25:

26:

27:

28:

29:

30:

31:

32:

33:

34:

35:

36:

37:

38:

39:

40:

41:

42:

43:

$44:$

45:

46:

47:

48:

49:

50:
SUBROUTINE DEFINE (INTYPE, ICHRS, INDAS, IREAD, ITIME, IEOR, IEIF, MEDIA, 2 TITIE, INSKIP, ISKIP, NCN!-, NTFORM)

$c$

c

CCMMON /ERRORS/ICRD, ISEG

INTEGER READ,TIME, EOR, $\subseteq O F, D A S, T F O R M$

DIMENSICN ICHPS (320), INDRS(22), IN $(80), \operatorname{INPUT}(10,38), \operatorname{READ}(10,56)$,

2 TIME $(10,68), \operatorname{ECR}(10,16), \operatorname{ECF}(10,28), D A S(10,22), \operatorname{ICHARS}(26)$,

3 TITLE (120), ISKIP(320), TFCRM $(1,6)$

DATA (INPUT $(1, J), J=1,38$ ) / $1 \mathrm{HI}, 1 \mathrm{HN}, 1 \mathrm{HP}, 1 \mathrm{HU}, 1 \mathrm{HT}, 1 \mathrm{H}=1 \mathrm{HT}, 1 \mathrm{HA}, 1 \mathrm{HP}, 1 \mathrm{HE}$, $21 H, 1 H, 1 H T, 1 H A, 1 H P, 1 H \equiv, 1 H, 1 H F, 1 H O, 1 H R, 1 H M, 1 H A, 1 H T, 1 H=, 1 H 7,1 H T$,

$31 \mathrm{HR}, 1 \mathrm{HA}, 1 \mathrm{HC}, 1 \mathrm{HK}, 8 * 1 \mathrm{H} /$

DATA (INPUT $(2, J), J=1,38) / 38 * 1 H$,

DATA (INPUT $(3, J), J=1,38$ ) / $1 \mathrm{HI}, 1 \mathrm{HN}, 1 \mathrm{HP}, 1 \mathrm{HU}, 1 \mathrm{HT}, 1 \mathrm{H}=, 1 \mathrm{HC}, 1 \mathrm{HA}, 1 \mathrm{HR}, 1 \mathrm{HD}$,

$21 \mathrm{H}, 1 \mathrm{H}, 1 \mathrm{HM}, 1 \mathrm{HA}, 1 \mathrm{HG}, 1 \mathrm{HE}, 1 \mathrm{HS}, 1 \mathrm{H,}, 1 \mathrm{H}, 1 \mathrm{HC}, 1 \mathrm{HH}, 1 \mathrm{HA}, 1 \mathrm{HN}, 1 \mathrm{HN}, 1 \mathrm{H}, 1 \mathrm{HL}$,

$31 \mathrm{HS}, 1 \mathrm{H}, 1 \mathrm{HP}, 1 \mathrm{HE}, 1 \mathrm{HR}, 1 \mathrm{H}, 1 \mathrm{H}_{-}^{\prime}, 1 \mathrm{HI}, 1 \mathrm{HN}, 1 \mathrm{H}, 1 \mathrm{H}=, 1 \mathrm{HE} /$

DATA (INPUT $(4, J), J=1,38) / 38 * 1 H$,

DATA ( INPUT $(5, J), J=1,38) / 1 H I, 1 H N, 1 H P, 1 H U, 1 H T, 1 H=, 1 H C, 1 H A, 1 H R, 1 H D$,

$21 \mathrm{H}, 1 \mathrm{HI}, 1 \mathrm{HM}, 1 \mathrm{HA}, 1 \mathrm{HG}, 1 \mathrm{HE}, 1 \mathrm{HS}, 1 \mathrm{H}, 1 \mathrm{H}, 1 \mathrm{HC}, 1 \mathrm{HH}, 1 \mathrm{HA}, 1 \mathrm{HN}, 1 \mathrm{HN}, 1 \mathrm{HE}, 1 \mathrm{HL}$,

$31 \mathrm{HS}, 1 \mathrm{H}, 1 \mathrm{HP}, 1 \mathrm{H} \equiv, 1 \mathrm{HR}, 1 \mathrm{H}, 1 \mathrm{HL}, 1 \mathrm{HI}, 1 \mathrm{HN}, 1 \mathrm{HE}, 1 \mathrm{H}=, 1 \mathrm{H} 4 /$

DATA (INPUT $(E, J), J=1,38$ ) /1HI, IHN, IHP, IHU, $1 H T, 1 H=, 1 H T, 1 H A, 1 H P, 1 H E$,

$21 \mathrm{H}, 1 \mathrm{H}, 1 \mathrm{HT}, 1 \mathrm{HA}, 1 \mathrm{HP}, 1 \mathrm{HE}, 1 \mathrm{H}, 1 \mathrm{HF}, 1 \mathrm{HO}, 1 \mathrm{HR}, 1 \mathrm{HM}, 1 \mathrm{HA}, 1 \mathrm{HT}, 1 \mathrm{H}=, 1 \mathrm{H} 7,1 \mathrm{HT}$,

$31 \mathrm{HR}, 1 \mathrm{HA}, 1 \mathrm{HC}, 1 \mathrm{HK}, 8 * 1 \mathrm{H}$,

DATA ( INPUT $(7, J), J=1,38$ ) / $1 \mathrm{HI}, 1 \mathrm{HN}, 1 \mathrm{HP}, 1 \mathrm{HU}, 1 \mathrm{HT}, 1 \mathrm{H}=, 1 \mathrm{HC}, 1 \mathrm{HA}, 1 \mathrm{HR}, 1 \mathrm{HD}$,

$21 \mathrm{H}, 1 \mathrm{HI}, 1 \mathrm{HM}, 1 \mathrm{HA}, 1 \mathrm{HG}, 1 \mathrm{HE}, 1 \mathrm{HS}, 1 \mathrm{H}, 1 \mathrm{H}, 1 \mathrm{HC}, 1 \mathrm{HH}, 1 \mathrm{HA}, 1 \mathrm{HN}, 1 \mathrm{HN}, 1 \mathrm{HE}, 1 \mathrm{HL}_{-}$,

$31 \mathrm{HS}, 1 \mathrm{H}, 1 \mathrm{HP}, 1 \mathrm{HE}, 1 \mathrm{HR}, 1 \mathrm{H}, 1 \mathrm{HL}, 1 \mathrm{HI}, 1 \mathrm{HN}, 1 \mathrm{HE}, 1 \mathrm{H}=, 1 \mathrm{H} 4 /$

DATA (INPUT $(8, J), J=1,38) / 1 \mathrm{HI}, 1 \mathrm{HN}, 1 \mathrm{HP}, 1 \mathrm{HU}, 1 \mathrm{HT}, 1 \mathrm{H}=, 1 \mathrm{HT}, 1 \mathrm{HA}, 1 \mathrm{HP}, 1 \mathrm{HE}$,

$21 H, 1 H, 1 H T, 1 H A, 1 H P, 1 H \equiv, 1 H, 1 H F, 1 H C, 1 H R, 1 H M, 1 H A, 1 H T, 1 H=, 1 H 7,1 H T$,

$31 \mathrm{HR}, 1 \mathrm{HA}, 1 \mathrm{HC}, 1 \mathrm{HK}, 8 \% 1 \mathrm{H} /$

DATA (INPUT $(9, J), J=1,38$ ) / $1 \mathrm{HI}, 1 \mathrm{HN}, 1 \mathrm{HP}, 1 \mathrm{HU}, 1 \mathrm{HT}, 1 \mathrm{H}=, 1 \mathrm{HC}, 1 \mathrm{HA}, 1 \mathrm{HR}, 1 \mathrm{HD}$,

$21 \mathrm{H}, 1 \mathrm{HI}, 1 \mathrm{HM}, 1 \mathrm{HA}, 1 \mathrm{HG}, 1 \mathrm{HE}, 1 \mathrm{HS}, 1 \mathrm{H}, 1 \mathrm{H}, 1 \mathrm{HC}, 1 \mathrm{HH}, 1 \mathrm{HA}, 1 \mathrm{HN}, 1 \mathrm{HN}, 1 \mathrm{H}, 1 \mathrm{HL}$,

$31 \mathrm{HS}, 1 \mathrm{H}, 1 \mathrm{HP}, 1 \mathrm{HE}, 1 \mathrm{HR}, 1 \mathrm{H}, 1 \mathrm{HL}, 1 \mathrm{HI}, 1 \mathrm{HN}, 1 \mathrm{H}, 1 \mathrm{H}=, 1 \mathrm{H} /$

DATA ( INPUT $(10, J), J=1,38$ ) $/ 1 \mathrm{HI}, 1 \mathrm{HN}, 1 \mathrm{HP}, 1 \mathrm{HU}, 1 \mathrm{HT}, 1 \mathrm{H}=, 1 \mathrm{HC}, 1 \mathrm{HA}, 1 \mathrm{HR}, 1 \mathrm{HD}$

$2,1 \mathrm{H}, 1 \mathrm{HI}, 1 \mathrm{HM}, 1 \mathrm{HA}, 1 \mathrm{HG}, 1 \mathrm{HE}, 1 \mathrm{HS}, 1 \mathrm{H}, 1 \mathrm{H}, 1 \mathrm{HC}, 1 \mathrm{HH}, 1 \mathrm{HA}, 1 \mathrm{HN}, 1 \mathrm{HN}, 1 \mathrm{H} \equiv, 1 \mathrm{HL}$,

$31 \mathrm{HS}, 1 \mathrm{H}, 1 \mathrm{HP}, 1 \mathrm{H}, 1 \mathrm{HR}, 1 \mathrm{H}, 1 \mathrm{HL}, 1 \mathrm{HI}, 1 \mathrm{HN}, 1 \mathrm{H} \equiv, 1 \mathrm{H}=, 1 \mathrm{H} /$

DATA (READ $(1, J), J=1,56) / 1 \mathrm{HP}, 1 \mathrm{HE}, 1 \mathrm{HA}, 1 \mathrm{HD}, 1 \mathrm{HI}, 1 \mathrm{HN}, 1 \mathrm{HG}, 1 \mathrm{H}=, 1 \mathrm{H}, 1 \mathrm{H}$,

$21 \mathrm{H}(, 1 \mathrm{HC}, 1 \mathrm{H}), 1 \mathrm{H}(, 1 \mathrm{H}+, 1 \mathrm{H} 1,1 \mathrm{H}+, 1 \mathrm{H+}, 1 \mathrm{H}-, 1 \mathrm{H} 2,1 \mathrm{H}-, 1 \mathrm{H}-, 1 \mathrm{HC}, 1 \mathrm{H}, 1 \mathrm{H}, 1 \mathrm{HC}$,

$31 \mathrm{H}), 1 \mathrm{H} 5,1 \mathrm{H*}, 1 \mathrm{H}(, 1 \mathrm{HV}, 1 \mathrm{H}), 1 \mathrm{H}(, 1 \mathrm{HE}, 1 \mathrm{H}), 1 \mathrm{H} 2,1 \mathrm{H}, 1 \mathrm{H}(, 1 \mathrm{HK}, 1 \mathrm{H}, 1 \mathrm{H}), 1 \mathrm{H}$,

$41 \mathrm{H}, 1 \mathrm{H}, 1 \mathrm{H}, 1 \mathrm{H}, 1 \mathrm{H}, 1 \mathrm{H}, 1 \mathrm{H}, 1 \mathrm{H}, 1 \mathrm{H}, 1 \mathrm{H}, 1 \mathrm{H}, 1 \mathrm{H}, 1 \mathrm{H}, 1 \mathrm{H}$,

DATA (READ $(2, J), J=1,56) / 1 H, 1 H, 1 H, 1 H, 1 H, 1 H, 1 H, 1 H, 1 H, 1 H$,

$21 \mathrm{H}, 1 \mathrm{H}, 1 \mathrm{H}, 1 \mathrm{H}, 1 \mathrm{H}, 1 \mathrm{H}, 1 \mathrm{H}, 1 \mathrm{H}, 1 \mathrm{H}, 1 \mathrm{H}, 1 \mathrm{H}, 1 \mathrm{H}, 1 \mathrm{H}, 1 \mathrm{H}, 1 \mathrm{H}, 1 \mathrm{H}$,

$31 \mathrm{H}, 1 \mathrm{H}, 1 \mathrm{H}, 1 \mathrm{H}, 1 \mathrm{H}, 1 \mathrm{H}, 1 \mathrm{H}, 1 \mathrm{H}, 1 \mathrm{H}, 1 \mathrm{H}, 1 \mathrm{H}, 1 \mathrm{H}, 1 \mathrm{H}, 1 \mathrm{H}, 1 \mathrm{H}, 1 \mathrm{H}$,

$41 \mathrm{H}, 1 \mathrm{H}, 1 \mathrm{H}, 1 \mathrm{H}, 1 \mathrm{H}, 1 \mathrm{H}, 1 \mathrm{H}, 1 \mathrm{H}, 1 \mathrm{H}, 1 \mathrm{H}, 1 \mathrm{H}, 1 \mathrm{H}, 1 \mathrm{H}, 1 \mathrm{H}$,

DATA (READ $(3, J), J=1,56) / 1 H R, 1 H E, 1 H A, 1 H D, 1 H I, 1 H N, 1 H G, 1 H=, 1 H 3,1 H *$,

$21 \mathrm{H}, 1 \mathrm{HC}, 1 \mathrm{H}), 1 \mathrm{H}(, 1 \mathrm{H}+, 1 \mathrm{H} 1,1 \mathrm{H}+, 1 \mathrm{H+}, 1 \mathrm{H}-, 1 \mathrm{H}, 1 \mathrm{H}-, 1 \mathrm{H}-, 1 \mathrm{HC}, 1 \mathrm{H}, 1 \mathrm{H}, 1 \mathrm{HO}$,

$31 \mathrm{H}), 1 \mathrm{H} 5,1 \mathrm{H}, 1 \mathrm{H}(, 1 \mathrm{HV}, 1 \mathrm{H}), 1 \mathrm{H}(, 1 \mathrm{HE}, 1 \mathrm{H}), 1 \mathrm{H} 2,1 \mathrm{H}, 1 \mathrm{H}(, 1 \mathrm{HK}, 1 \mathrm{H}, 1 \mathrm{H}), 1 \mathrm{H}$,

$41 \mathrm{H}, 1 \mathrm{H}, 1 \mathrm{H}, 1 \mathrm{H}, 1 \mathrm{H}, 1 \mathrm{H}, 1 \mathrm{H}, 1 \mathrm{H}, 1 \mathrm{H}, 1 \mathrm{H}, 1 \mathrm{H}, 1 \mathrm{H}, 1 \mathrm{H}, 1 \mathrm{H}$,

DATA $(\operatorname{READ}(4, J), J=1,56) / 1 \mathrm{H}, 1 \mathrm{H}, 1 \mathrm{H}, 1 \mathrm{H}, 1 \mathrm{H}, 1 \mathrm{H}, 1 \mathrm{H}, 1 \mathrm{H}, 1 \mathrm{H}, 1 \mathrm{H}$,

$21 \mathrm{H}, 1 \mathrm{H}, 1 \mathrm{H}, 1 \mathrm{H}, 1 \mathrm{H}, 1 \mathrm{H}, 1 \mathrm{H}, 1 \mathrm{H}, 1 \mathrm{H}, 1 \mathrm{H}, 1 \mathrm{H}, 1 \mathrm{H}, 1 \mathrm{H}, 1 \mathrm{H}, 1 \mathrm{H}, 1 \mathrm{H}$, 
51:

52:

53:

54:

55:

56:

$57:$

58:

59:

60:

61:

62:

63:

64:

$65:$

66:

67:

68:

69:

70 :

$71:$

$72:$

73:

$74:$

$75:$

76 :

$77:$

78 :

79:

80:

81:

82:

83:

84:

85:

86:

87:

88:

89:

90:

91:

92:

93:

$94:$

95:

96:

97:

98 :

99:

100:

$31 H, 1 H, 1 H, 1 H, 1 H, 1 H, 1 H, 1 H, 1 H, 1 H, 1 H, 1 H, 1 H, 1 H, 1 H, 2 H$,

$41 \mathrm{1H}, 1 \mathrm{H}, 1 \mathrm{H}, 1 \mathrm{H}, 1 \mathrm{H}, 1 \mathrm{H}, 1 \mathrm{H}, 1 \mathrm{H}, 1 \mathrm{H}, 1 \mathrm{H}, 1 \mathrm{H}, 1 \mathrm{H}, 1 \mathrm{H}, 1 \mathrm{H}$,

DATA (READ $(5, J), J=1,56) / 1 \mathrm{HR}, 1 \mathrm{HE}, 1 \mathrm{HA}, 1 \mathrm{HD}, 1 \mathrm{HI}, 1 \mathrm{HN}, 1 \mathrm{HG}, 1 \mathrm{H}=, 1 \mathrm{H}, 1 \mathrm{HN}$,

$21 H), 1 H(, 1 H K, 1 H, 1 H), 1 H 3,1 H *, 1 H(, 1 H C, 1 H), 1 H(, 1 H K, 1 H, 1 H), 1 H(, 1 H R$,

$31 \mathrm{HO}, 1 \mathrm{H}, 1 \mathrm{H}, 1 \mathrm{H}, 1 \mathrm{H}, 1 \mathrm{H}(, 1 \mathrm{HR}, 1 \mathrm{H}), 1 \mathrm{H}, 1 \mathrm{H}, 1 \mathrm{H}, 1 \mathrm{HA}, 1 \mathrm{H}, 1 \mathrm{H}, 1 \mathrm{HK}, 1 \mathrm{H}$,

$41 H, 1 H, 1 H, 1 H, 1 H, 1 H, 1 H, 1 H, 1 H, 1 H, 1 H, 1 H, 1 H, 1 H /$

DATA (READ $(6, J), J=1,56) / 1 \mathrm{HR}, 1 \mathrm{HE}, 1 \mathrm{HA}, 1 \mathrm{HD}, 1 \mathrm{HI}, 1 \mathrm{HN}, 1 \mathrm{HG}, 1 \mathrm{H}=, 1 \mathrm{H}, 1 \mathrm{HK}$,

$21 H C, 1 H), 1 H 3,1 H *, 1 H(, 1 H C, 1 H), 1 H(, 1 H K, 1 H, 1 H), 1 H(, 1 H K, 1 H, 1 H,, 1 H *$,

$31 \mathrm{HN}, 1 \mathrm{H}, 01 \mathrm{H}, 1 \mathrm{H}+, 1 \mathrm{H}, 1 \mathrm{H}=, 1 \mathrm{H}, 1 \mathrm{H}, 1 \mathrm{H}, 1 \mathrm{H}, 1 \mathrm{H}, 1 \mathrm{HR}, 1 \mathrm{HC}, 1 \mathrm{H}, 1 \mathrm{H}, 1 \mathrm{H}$,

$41 \mathrm{HK}, 1 \mathrm{H}, 1 \mathrm{H}), 1 \mathrm{H}, \mathrm{,}-1 \mathrm{H}, 1 \mathrm{H}(, 1 \mathrm{HA}, 1 \mathrm{H}), 1 \mathrm{H}, 1 \mathrm{H}, 1 \mathrm{H}(, 1 \mathrm{HK}, 1 \mathrm{H}, 1 \mathrm{H})$

DATA (READ (7,J), J=1,56)/1HR, $1 \mathrm{HE}, 1 \mathrm{HA}, 1 \mathrm{HD}, 1 \mathrm{HI}, 1 \mathrm{HN}, 1 \mathrm{HG}, 1 \mathrm{H}=, 1 \mathrm{H}, 1 \mathrm{HK}$,

$21 H C, 1 H), 1 H 3,1 H *, 1 H(, 1 H C, 1 H), 1 H(, 1 H K, 1 H, 1 H), 1 H(, 1 H K, 1 H, 1 H J, 1 H *$,

$31 \mathrm{HN}, 1 \mathrm{H}, 1 \mathrm{H}(, 1 \mathrm{H}+, 1 \mathrm{H}, 1 \mathrm{H}=, 1 \mathrm{H}=, 1 \mathrm{H}, 1 \mathrm{H}, 1 \mathrm{H}, 1 \mathrm{H}, 1 \mathrm{HR}, 1 \mathrm{HC}, 1 \mathrm{H}, 1 \mathrm{H}, 1 \mathrm{H}($,

$41 H K, 1 H, 1 H), 1 H 2,1 H *, 1 H(, 1 H A, 1 H), 1 H 2,1 H *, 1 H(, 1 H K, 1 H, 1 H) /$

DATA (READ $(8, J), J=1,56) / 1 H R, 1 H E, 1 H A, 1 H D, 1 H I, 1 H N, 1 H G, 1 H=, 1 H 3,1 H *$,

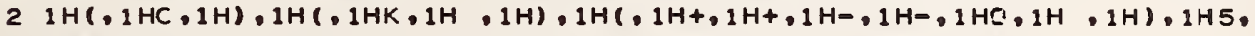

$31 H *, 1 H(, 1 H V, 1 H C, 1 H, 1 H), 1 H(, 1 H V, 1 H K, 1 H, 1 H), 1 H 3,1 H *, 1 H(, 1 H A, 1 H)$,

$41 \mathrm{H}, 1 \mathrm{HE}, 1 \mathrm{HO}, 1 \mathrm{H}, 1 \mathrm{H}), 1 \mathrm{H}, 1 \mathrm{HK}, 1 \mathrm{HX}, 1 \mathrm{H}, 1 \mathrm{H}, 1 \mathrm{H}, 1 \mathrm{H}, 1 \mathrm{H}, 1 \mathrm{H}$,

DATA (READ $(9, J), J=1,56) / 1 H R, 1 H E, 1 H A, 1 H D, 1 H I, 1 H N, 1 H G, 1 H=, 1 H 3,1 H *$,

$21 H(, 1 H C, 1 H), 1 H(, 1 H K, 1 H, 1 H), 1 H(, 1 H+, 1 H+, 1 H=, 1 H=, 1 H C, 1 H, 1 H), 1 H 5$,

$31 H *, 1 H(, 1 H V, 1 H O, 1 H, 1 H), 1 H(, 1 H V, 1 H K, 1 H, 1 H), 1 H 3,1 H *, 1 H(, 1 H A, 1 H)$,

$41 \mathrm{H}, 1 \mathrm{HE}, 1 \mathrm{HD}, 1 \mathrm{H}, 1 \mathrm{H}), 1 \mathrm{H}, 1 \mathrm{HK}, 1 \mathrm{HX}, 1 \mathrm{H}), 1 \mathrm{H}, 1 \mathrm{H}, 1 \mathrm{H}, 1 \mathrm{H}, 1 \mathrm{H}$,

DATA (READ $(10, \mathrm{~J}), \mathrm{J}=1,56) / 1 \mathrm{HR}, 1 \mathrm{HE}, 1 \mathrm{HA}, 1 \mathrm{HD}, 1 \mathrm{HI}, 1 \mathrm{HN}, 1 \mathrm{HG}, 1 \mathrm{H}=, 1 \mathrm{H}, 1 \mathrm{HC}$,

$21 H *, 1 H K, 1 H, 1 H), 1 H 3,1 H *, 1 H(, 1 H C, 1 H), 1 H(, 1 H+, 1 H, 1 H-, 1 H-, 1 H), 1 H 6$,

$31 H *, 1 H(, 1 H R, 1 H), 1 H(, 1 H A, 1 H), 1 H 2,1 H *, 1 H(, 1 H K, 1 H, 1 H), 1 H, 1 H, 1 H$,

$41 \mathrm{H}, 1 \mathrm{H}, 1 \mathrm{H}, 1 \mathrm{H}, 1 \mathrm{H}, 1 \mathrm{H}, 1 \mathrm{H}, 1 \mathrm{H}, 1 \mathrm{H}, 1 \mathrm{H}, 1 \mathrm{H}, 1 \mathrm{H}, 1 \mathrm{H}, 1 \mathrm{H}$,

DATA (TIME $(1, J), J=1,68) / 1 \mathrm{HT}, 1 \mathrm{HI}, 1 \mathrm{HM}, 1 \mathrm{HE}, 1 \mathrm{H}=, 1 \mathrm{H}, 1 \mathrm{H*}, 1 \mathrm{H}(, 1 \mathrm{HS}, 1 \mathrm{H})$, $21 \mathrm{H}, 1 \mathrm{HK}, 1 \mathrm{H}, 1 \mathrm{H}), 1 \mathrm{H}, 1 \mathrm{H}, 1 \mathrm{H}(, 1 \mathrm{HS}, 1 \mathrm{H}, 1 \mathrm{H}, 1 \mathrm{H},, 1 \mathrm{H}, 1 \mathrm{HK}, 1 \mathrm{H}, 1 \mathrm{H}), 1 \mathrm{H}$,

$31 \mathrm{H}, 1 \mathrm{H}, 1 \mathrm{H}, 1 \mathrm{H}, 1 \mathrm{H}, 1 \mathrm{H}, 1 \mathrm{H}, 1 \mathrm{H}, 1 \mathrm{H}, 1 \mathrm{H}, 1 \mathrm{H}, 1 \mathrm{H}, 1 \mathrm{H}, 1 \mathrm{H}, 1 \mathrm{H}, 1 \mathrm{H}$,

$41 \mathrm{H}, 1 \mathrm{H}, 1 \mathrm{H}, 1 \mathrm{H}, 1 \mathrm{H}, 1 \mathrm{H}, 1 \mathrm{H}, 1 \mathrm{H}, 1 \mathrm{H}, 1 \mathrm{H}, 1 \mathrm{H}, 1 \mathrm{H}, 1 \mathrm{H}, 1 \mathrm{H}, 1 \mathrm{H}, 1 \mathrm{H}$,

$51 \mathrm{H}, 1 \mathrm{H}, 1 \mathrm{H}, 1 \mathrm{H}, 1 \mathrm{H}, 1 \mathrm{H}, 1 \mathrm{H}, 1 \mathrm{H}, 1 \mathrm{H}, 1 \mathrm{H} /$

DATA (TIME $(2, J), J=1,68) / 1 H, 1 H, 1 H, 1 H, 1 H, 1 H, 1 H, 1 H, 1 H, 1 H$,

$21 \mathrm{H}, 1 \mathrm{H}, 1 \mathrm{H}, 1 \mathrm{H}, 1 \mathrm{H}, 1 \mathrm{H}, 1 \mathrm{H}, 1 \mathrm{H}, 1 \mathrm{H}, 1 \mathrm{H}, 1 \mathrm{H}, 1 \mathrm{H}, 1 \mathrm{H}, 1 \mathrm{H}, 1 \mathrm{H}, 1 \mathrm{H}$,

$31 \mathrm{H}, 1 \mathrm{H}, 1 \mathrm{H}, 1 \mathrm{H}, 1 \mathrm{H}, 1 \mathrm{H}, 1 \mathrm{H}, 1 \mathrm{H}, 1 \mathrm{H}, 1 \mathrm{H}, 1 \mathrm{H}, 1 \mathrm{H}, 1 \mathrm{H}, 1 \mathrm{H}, 1 \mathrm{H}, 1 \mathrm{H}$,

$41 H, 1 H, 1 H, 1 H, 1 H, 1 H, 1 H, 1 H, 1 H, 1 H, 1 H, 1 H, 1 H, 1 H, 1 H, 1 H$, $51 H, 1 H, 1 H, 1 H, 1 H, 1 H, 1 H, 1 H, 1 H, 1 H$,

DATA (TIME $(3, J), J=1,68) / 1 H T, 1 H I, 1 H M, 1 H E, 1 H=, 1 H 3,1 H *, 1 H(, 1 H S, 1 H)$, $21 \mathrm{H}, 1 \mathrm{HK}, 1 \mathrm{H}, 1 \mathrm{H}), 1 \mathrm{H}, 1 \mathrm{H*}, 1 \mathrm{H}, 1 \mathrm{HS}, 1 \mathrm{H}), 1 \mathrm{H}, 1 \mathrm{H}, 1 \mathrm{H}(, 1 \mathrm{HK}, 1 \mathrm{H}, 1 \mathrm{H}), 1 \mathrm{H}$, $31 \mathrm{H}, 1 \mathrm{H}, 1 \mathrm{H}, 1 \mathrm{H}, 1 \mathrm{H}, 1 \mathrm{H}, 1 \mathrm{H}, 1 \mathrm{H}, 1 \mathrm{H}, 1 \mathrm{H}, 1 \mathrm{H}, 1 \mathrm{H}, 1 \mathrm{H}, 1 \mathrm{H}, 1 \mathrm{H}, 1 \mathrm{H}$, $41 \mathrm{H}, 1 \mathrm{H}, 1 \mathrm{H}, 1 \mathrm{H}, 1 \mathrm{H}, 1 \mathrm{H}, 1 \mathrm{H}, 1 \mathrm{H}, 1 \mathrm{H}, 1 \mathrm{H}, 1 \mathrm{H}, 1 \mathrm{H}, 1 \mathrm{H}, 1 \mathrm{H}, 1 \mathrm{H}, 1 \mathrm{H}$, $51 \mathrm{H}, 1 \mathrm{H}, 1 \mathrm{H}, 1 \mathrm{H}, 1 \mathrm{H}, 1 \mathrm{H}, 1 \mathrm{H}, 1 \mathrm{H}, 1 \mathrm{H}, 1 \mathrm{H}$,

DATA (TIME $(4, J), J=1,68) / 1 H, 1 H, 1 H, 1 H, 1 H, 1 H, 1 H, 1 H, 1 H, 1 H$, $21 \mathrm{H}, 1 \mathrm{H}, 1 \mathrm{H}, 1 \mathrm{H}, 1 \mathrm{H}, 1 \mathrm{H}, 1 \mathrm{H}, 1 \mathrm{H}, 1 \mathrm{H}, 1 \mathrm{H}, 1 \mathrm{H}, 1 \mathrm{H}, 1 \mathrm{H}, 1 \mathrm{H}, 1 \mathrm{H}, 1 \mathrm{H}$, $31 \mathrm{H}, 1 \mathrm{H}, 1 \mathrm{H}, 1 \mathrm{H}, 1 \mathrm{H}, 1 \mathrm{H}, 1 \mathrm{H}, 1 \mathrm{H}, 1 \mathrm{H}, 1 \mathrm{H}, 1 \mathrm{H}, 1 \mathrm{H}, 1 \mathrm{H}, 1 \mathrm{H}, 1 \mathrm{H}, 1 \mathrm{H}$, $41 H, 1 H, 1 H, 1 H, 1 H, 1 H, 1 H, 1 H, 1 H, 1 H, 1 H, 1 H, 1 H, 1 H, 1 H, 1 H$, $51 \mathrm{H}, 1 \mathrm{H}, 1 \mathrm{H}, 1 \mathrm{H}, 1 \mathrm{H}, 1 \mathrm{H}, 1 \mathrm{H}, 1 \mathrm{H}, 1 \mathrm{H}, 1 \mathrm{H}$,

DATA (TIME $(5, J), J=1,68) / 1 \mathrm{HT}, 1 \mathrm{HI}, 1 \mathrm{HM}, 1 \mathrm{HE}, 1 \mathrm{H}=, 1 \mathrm{H}, 1 \mathrm{H}, 1 \mathrm{H}(, 1 \mathrm{HH}, 1 \mathrm{H})$, $21 \mathrm{H}, 1 \mathrm{HK}, 1 \mathrm{H}:, 1 \mathrm{H}), 1 \mathrm{H}, 1 \mathrm{H}, 1 \mathrm{H}(, 1 \mathrm{HM}, 1 \mathrm{H}), 1 \mathrm{H}, 1 \mathrm{H}, 1 \mathrm{H}, 1 \mathrm{H}(, 1 \mathrm{HK}, 1 \mathrm{H}, 1 \mathrm{H})$, $31 \mathrm{H}, 1 \mathrm{H}, 1 \mathrm{H}, 1 \mathrm{H}, 1 \mathrm{H}, 1 \mathrm{H}, 1 \mathrm{H}, 1 \mathrm{H}, 1 \mathrm{H}, 1 \mathrm{H}, 1 \mathrm{H}, 1 \mathrm{H}, 1 \mathrm{H}, 1 \mathrm{H}, 1 \mathrm{H}, 1 \mathrm{H}$, $41 \mathrm{H}, 1 \mathrm{H}, 1 \mathrm{H}, 1 \mathrm{H}, 1 \mathrm{H}, 1 \mathrm{H}, 1 \mathrm{H}, 1 \mathrm{H}, 1 \mathrm{H}, 1 \mathrm{H}, 1 \mathrm{H}, 1 \mathrm{H}, 1 \mathrm{H}, 1 \mathrm{H}, 1 \mathrm{H}, 1 \mathrm{H}$, 
101:

$102:$

103:

104:

105:

106:

$107:$

108:

109:

110 :

$111:$

112 :

$113:$

114 :

115 :

116 :

117 :

$118:$

$119:$

120 :

121 :

122 :

123 :

$124:$

125 :

126 :

$127:$

128 :

129 :

130 :

$131:$

132 :

$133:$

$134:$

135 :

$136:$

137 :

138 :

$139:$

140 :

141:

142 :

143:

144:

145 :

146 :

147 :

148:

149:

150:
$51 \mathrm{H}, 1 \mathrm{H}, 1 \mathrm{H}, 1 \mathrm{H}, 1 \mathrm{H}, 1 \mathrm{H}, 1 \mathrm{H}, 1 \mathrm{H}, 1 \mathrm{H}, 1 \mathrm{H}$,

DATA (TIME $(6, J), J=1,68) / 1 \mathrm{HT}, 1 \mathrm{HI}, 1 \mathrm{HM}, 1 \mathrm{HE}, 1 \mathrm{H}=, 1 \mathrm{H} 4,1 \mathrm{H}, 1 \mathrm{H}(, 1 \mathrm{HK}, 1 \mathrm{H}-$, $21 \mathrm{HN}, 1 \mathrm{H}), 1 \mathrm{H} 2,1 \mathrm{H}, 1 \mathrm{H}(, 1 \mathrm{HK}, 1 \mathrm{H}, 1 \mathrm{H}), 1 \mathrm{H} 2,1 \mathrm{H}, 1 \mathrm{H}, 1 \mathrm{HD}, 1 \mathrm{H}), 1 \mathrm{H}(, 1 \mathrm{HK}, 1 \mathrm{H}$,

$31 \mathrm{H}), 1 \mathrm{H} 2,1 \mathrm{H} * 1 \mathrm{H}(, 1 \mathrm{HH}, 1 \mathrm{H}), 1 \mathrm{H}(, 1 \mathrm{HK}, 1 \mathrm{H}:, 1 \mathrm{H}), 1 \mathrm{H} 2,1 \mathrm{H} *, 1 \mathrm{H}(, 1 \mathrm{HM}, 1 \mathrm{H}), 1 \mathrm{H}($,

$41 \mathrm{HK}, 1 \mathrm{H}:, 1 \mathrm{H}), 1 \mathrm{H} 2,1 \mathrm{H} *, 1 \mathrm{H}(, 1 \mathrm{HS}, 1 \mathrm{H}), 1 \mathrm{H}(, 1 \mathrm{HK}, 1 \mathrm{H}, 1 \mathrm{H}), 1 \mathrm{H}(, 1 \mathrm{HK}, 1 \mathrm{HH}, 1 \mathrm{H})$,

$51 \mathrm{H}(, 1 \mathrm{HK}, 1 \mathrm{HR}, 1 \mathrm{H}), 1 \mathrm{H}, 1 \mathrm{H}, 1 \mathrm{H}(, 1 \mathrm{HK}, 1 \mathrm{H}, 1 \mathrm{H}) /$

DATA (TIME $(7, \mathrm{~J}), \mathrm{J}=1,68) / 1 \mathrm{H}^{\top}, 1 \mathrm{HI}, 1 \mathrm{HM}, 1 \mathrm{HE}, 1 \mathrm{H}=, 1 \mathrm{H} 4,1 \mathrm{H}^{*}, 1 \mathrm{H}, 1 \mathrm{HK}, 1 \mathrm{H}$, $21 \mathrm{HN}, 1 \mathrm{H}), 1 \mathrm{H} 2,1 \mathrm{H}, 1 \mathrm{H}(, 1 \mathrm{HK}, 1 \mathrm{H}, 1 \mathrm{H}), 1 \mathrm{H}, 1 \mathrm{H} *, 1 \mathrm{H}(, 1 \mathrm{HD}, 1 \mathrm{H}), 1 \mathrm{H}(, 1 \mathrm{HK}, 1 \mathrm{H}$,

$31 \mathrm{H}), 1 \mathrm{H2}, 1 \mathrm{H*}, 1 \mathrm{H}(, 1 \mathrm{HH}, 1 \mathrm{H}), 1 \mathrm{H}(, 1 \mathrm{HK}, 1 \mathrm{H}: 1 \mathrm{H}), 1 \mathrm{H} 2,1 \mathrm{H}, 1 \mathrm{H}(, 1 \mathrm{HM}, 1 \mathrm{H}), 1 \mathrm{H}$,

$41 \mathrm{HK}, 1 \mathrm{H}:, 1 \mathrm{H}), 1 \mathrm{H}, 1 \mathrm{H}, 1 \mathrm{H}(, 1 \mathrm{HS}, 1 \mathrm{H}), 1 \mathrm{H}(, 1 \mathrm{HK}, 1 \mathrm{H}, 1 \mathrm{H}), 1 \mathrm{H}(, 1 \mathrm{HK}, 1 \mathrm{HH}, 1 \mathrm{H})$,

$51 \mathrm{H}(, 1 \mathrm{HK}, 1 \mathrm{HR}, 1 \mathrm{H}), 1 \mathrm{H}, 1 \mathrm{H}, 1 \mathrm{H}(, 1 \mathrm{HK}, 1 \mathrm{H}, 1 \mathrm{H}) /$

DATA (TIME $(8, J), J=1,68) / 1 H T, 1 H I, 1 H M, 1 H E, 1 H=, 1 H 3,1 H *, 1 H(, 1 H D, 1 H)$,

$21 \mathrm{H}, 1 \mathrm{H}, 1 \mathrm{H}(, 1 \mathrm{HH}, 1 \mathrm{H}), 1 \mathrm{H}(, 1 \mathrm{HK}, 1 \mathrm{H}:, 1 \mathrm{H}), 1 \mathrm{H} 2,1 \mathrm{H} *, 1 \mathrm{H}(, 1 \mathrm{HM}, 1 \mathrm{H}), 1 \mathrm{H}(, 1 \mathrm{HK}$,

$31 \mathrm{H}:, 1 \mathrm{H}), 1 \mathrm{H} 2,1 \mathrm{H*}, 1 \mathrm{H}(, 1 \mathrm{HS}, 1 \mathrm{H}), 1 \mathrm{H} 4,1 \mathrm{H} *, 1 \mathrm{H}(, 1 \mathrm{HK}, 1 \mathrm{H}, 1 \mathrm{H}), 1 \mathrm{H}(, 1 \mathrm{HK}, 1 \mathrm{HX}$,

$41 \mathrm{H}, 1 \mathrm{H}, 1 \mathrm{H}, 1 \mathrm{H}, 1 \mathrm{H}, 1 \mathrm{H}, 1 \mathrm{H}, 1 \mathrm{H}, 1 \mathrm{H}, 1 \mathrm{H}, 1 \mathrm{H}, 1 \mathrm{H}, 1 \mathrm{H}, 1 \mathrm{H}, 1 \mathrm{H}, 1 \mathrm{H}$,

$51 \mathrm{H}, 1 \mathrm{H}, 1 \mathrm{H}, 1 \mathrm{H}, 1 \mathrm{H}, 1 \mathrm{H}, 1 \mathrm{H}, 1 \mathrm{H}, 1 \mathrm{H}, 1 \mathrm{H}$,

DATA (TIME $(9, J), J=1,68) / 1 H T, 1 H I, 1 H M, 1 H E, 1 H=, 1 H 3,1 H *, 1 H(, 1 H D, 1 H)$, $21 \mathrm{H} 2,1 \mathrm{H} *, 1 \mathrm{H}(, 1 \mathrm{HH}, 1 \mathrm{H}), 1 \mathrm{H}(, 1 \mathrm{HK}, 1 \mathrm{H}:, 1 \mathrm{H}), 1 \mathrm{H} 2,1 \mathrm{H}, 1 \mathrm{H}(, 1 \mathrm{HM}, 1 \mathrm{H}), 1 \mathrm{H}, 1 \mathrm{HK}$,

$31 \mathrm{H}:, 1 \mathrm{H}), 1 \mathrm{H}, 1 \mathrm{H*}, 1 \mathrm{H}(, 1 \mathrm{HS}, 1 \mathrm{H}), 1 \mathrm{H}, 1 \mathrm{H} *, 1 \mathrm{H}(, 1 \mathrm{HK}, 1 \mathrm{H}, 1 \mathrm{H}), 1 \mathrm{H}, 1 \mathrm{HK}, 1 \mathrm{HX}$,

$41 \mathrm{H}, 1 \mathrm{H}, 1 \mathrm{H}, 1 \mathrm{H}, 1 \mathrm{H}, 1 \mathrm{H}, 1 \mathrm{H}, 1 \mathrm{H}, 1 \mathrm{H}, 1 \mathrm{H}, 1 \mathrm{H}, 1 \mathrm{H}, 1 \mathrm{H}, 1 \mathrm{H}, 1 \mathrm{H}, 1 \mathrm{H}$,

$51 \mathrm{H}, 1 \mathrm{H}, 1 \mathrm{H}, 1 \mathrm{H}, 1 \mathrm{H}, 1 \mathrm{H}, 1 \mathrm{H}, 1 \mathrm{H}, 1 \mathrm{H}, 1 \mathrm{H} /$

DATA (TIME $(10, \mathrm{~J}), \mathrm{J}=1,68) / 1 \mathrm{HT}, 1 \mathrm{HI}, 1 \mathrm{HM}, 1 \mathrm{HE}, 1 \mathrm{H}=, 1 \mathrm{H}(, 1 \mathrm{HK}, 1 \mathrm{H}, 1 \mathrm{H}), 1 \mathrm{H} 1$, $21 \mathrm{H}, 1 \mathrm{H*}, 1 \mathrm{H}(, 1 \mathrm{HK}, 1 \mathrm{H}, 1 \mathrm{HN}, 1 \mathrm{H}), 1 \mathrm{H}, 1 \mathrm{H}, 1 \mathrm{H}(, 1 \mathrm{HH}, 1 \mathrm{H}), 1 \mathrm{H}(, 1 \mathrm{HK}, 1 \mathrm{H}, 1 \mathrm{H})$,

$31 \mathrm{H}, 1 \mathrm{H}^{*}, 1 \mathrm{H}(, 1 \mathrm{H}, 1 \mathrm{H}), 1 \mathrm{H}(, 1 \mathrm{HK}, 1 \mathrm{H}, 1 \mathrm{H}), 1 \mathrm{H} 2,1 \mathrm{H}, 1 \mathrm{H}(, 1 \mathrm{HS}, 1 \mathrm{H}), 1 \mathrm{H}(, 1 \mathrm{HK}$,

$41 \mathrm{H}, 1 \mathrm{H}), 1 \mathrm{H}(, 1 \mathrm{HK}, 1 \mathrm{HH}, 1 \mathrm{H}), 1 \mathrm{H} 4,1 \mathrm{H}, 1 \mathrm{H}, 1 \mathrm{H}(, 1 \mathrm{HK}, 1 \mathrm{H}, 1 \mathrm{H}, 1 \mathrm{H}, 1 \mathrm{H}, 1 \mathrm{H}$,

$51 \mathrm{H}, 1 \mathrm{H}, 1 \mathrm{H}, 1 \mathrm{H}, 1 \mathrm{H}, 1 \mathrm{H}, 1 \mathrm{H}, 1 \mathrm{H}, 1 \mathrm{H}, 1 \mathrm{H} /$

DATA (EOR $(1, J), J=1,16) / 1 H E, 1 H C, 1 H R, 1 H=, 1 H E, 1 H C, 1 H R, 1 H, 1 H, 1 H$,

$21 \mathrm{H}, 1 \mathrm{H}, 1 \mathrm{H}, 1 \mathrm{H}, 1 \mathrm{H}, 1 \mathrm{H}$,

DATA $(E O R(2, J), J=1,16) / 1 H, 1 H, 1 H, 1 H, 1 H, 1 H, 1 H, 1 H, 1 H, 1 H$,

$21 \mathrm{H}, 1 \mathrm{H}, 1 \mathrm{H}, 1 \mathrm{H}, 1 \mathrm{H}, 1 \mathrm{H}$,

DATA (ECR(3,J),J=1,16) /1HE, 1HC, 1HR, 1H=, 1H(, 1HK, 1H, 1H), 1H(, 1HK, $21 \mathrm{HX}, 1 \mathrm{H}), 1 \mathrm{H}, 1 \mathrm{H}, 1 \mathrm{H}, 1 \mathrm{H}$,

DATA (ECR(4,J), J =1,16) /1H, 1H, 1H, 1H, $1 \mathrm{H}, 1 \mathrm{H}, 1 \mathrm{H}, 1 \mathrm{H}, 1 \mathrm{H}, 1 \mathrm{H}$, $21 \mathrm{H}, 1 \mathrm{H}, 1 \mathrm{H}, 1 \mathrm{H}, 1 \mathrm{H}, 1 \mathrm{H}$,

DATA (ECR $(5, J), J=1,16) / 1 H E, 1 H C, 1 H R, 1 H=, 1 H 8,1 H 0,1 H *, 1 H(, 1 H K, 1 H$,

$21 \mathrm{H}, 1 \mathrm{H}, 1 \mathrm{H}, 1 \mathrm{H}, 1 \mathrm{H}, 1 \mathrm{H}$,

DATA (EQR $(6, J), J=1,16) / 1 H E, 1 H C, 1 H R, 1 H=, 1 H E, 1 H O, 1 H R, 1 H, 1 H, 1 H$,

$21 \mathrm{H}, 1 \mathrm{H}, 1 \mathrm{H}, 1 \mathrm{H}, 1 \mathrm{H}, 1 \mathrm{H}$,

DATA (EOR $(7, J), J=1,16) / 1 H E, 1 H C, 1 H R, 1 H=, 1 H(, 1 H K, 1 H, 1 H), 1 H(, 1 H K$, $21 \mathrm{HX}, 1 \mathrm{H}), 1 \mathrm{H}, 1 \mathrm{H}, 1 \mathrm{H}, 1 \mathrm{H} /$

DATA (EOR $(8, J), J=1,16) / 1 H E, 1 H C, 1 H R, 1 H=, 1 H E, 1 H C, 1 H R, 1 H, 1 H, 1 H$,

$21 \mathrm{H}, 1 \mathrm{H}, 1 \mathrm{H}, 1 \mathrm{H}, 1 \mathrm{H}, 1 \mathrm{H} /$

DATA $(E O R(9, J), J=1,16) / 1 H E, 1 H C, 1 H R, 1 H=, 1 H(, 1 H K, 1 H, 1 H), 1 H(, 1 H K$,

$21 H X, 1 H), 1 H(, 1 H K, 1 H, 1 H) /$

DATA (EOR $(10, J), J=1,16) / 1 H E, 1 H C, 1 H R, 1 H=, 1 H 7,1 H O, 1 H *, 1 H(, 1 H K, 1 H$, $21 \mathrm{H}), 1 \mathrm{H}, 1 \mathrm{H}, 1 \mathrm{H}, 1 \mathrm{H}, 1 \mathrm{H}$,

DATA (ECF $(1, \mathrm{~J}), \mathrm{J}=1,28) / 1 \mathrm{HE}, 1 \mathrm{HO}, 1 \mathrm{HF}, 1 \mathrm{H}=, 1 \mathrm{HE}, 1 \mathrm{HC}, 1 \mathrm{HF}, 1 \mathrm{H}, 1 \mathrm{H}, 1 \mathrm{H}$,

$21 \mathrm{H}, 1 \mathrm{H}, 1 \mathrm{H}, 1 \mathrm{H}, 1 \mathrm{H}, 1 \mathrm{H}, 1 \mathrm{H}, 1 \mathrm{H}, 1 \mathrm{H}, 1 \mathrm{H}, 1 \mathrm{H}, 1 \mathrm{H}, 1 \mathrm{H}, 1 \mathrm{H}, 1 \mathrm{H}, 1 \mathrm{H}$, $31 \mathrm{H}, 1 \mathrm{H} /$

DATA $(E C F(2, J), J=1,28) / 1 H, 1 H, 1 H, 1 H, 1 H, 1 H, 1 H, 1 H, 1 H, 1 H$, 
151:

152:

153:

$154:$

155:

$156:$

157:

158:

$159:$

$160:$

$1 \in 1$ :

162 :

163:

$1 \in 4:$

165:

166 :

167:

168 :

$169:$

170:

171:

172:

173:

174:

175:

176:

177:

178 :

179:

180 :

181:

182 :

$183:$

$184:$

185:

136 :

187:

188 :

$189:$

190 :

191:

192:

193:

194:

195:

196 :

$197:$

198 :

199:

200:
$21 H, 1 H, 1 H, 1 H, 1 H, 1 H, 1 H, 1 H, 1 H, 1 H, 1 H, 1 H, 1 H, 1 H, 1 H, 1 H$,

$31 \mathrm{H}, 1 \mathrm{H}$,

DATA (EOF $(3, J), J=1,28) / 1 H E, 1 H C, 1 H F, 1 H=, 1 H(, 1 H K, 1 H F, 1 H), 1 H(, 1 H K$,

$21 H I, 1 H), 1 H(, 1 H K, 1 H L, 1 H), 1 H(, 1 H K, 1 H E, 1 H), 1 H(, 1 H K, 1 H N, 1 H), 1 H(, 1 H K$,

$31 H D, 1 H) /$

DATA (EOF $(4, J), J=1,28) / 1 H, 1 H, 1 H, 1 H, 1 H, 1 H, 1 H, 1 H, 1 H, 1 H$,

$21 H, 1 H, 1 H, 1 H, 1 H, 1 H, 1 H, 1 H, 1 H, 1 H, 1 H, 1 H, 1 H, 1 H, 1 H, 1 H$,

$31 \mathrm{H}, 1 \mathrm{H} /$

DATA (EOF $(5, J), J=1,28) / 1 \mathrm{HE}, 1 \mathrm{HO}, 1 \mathrm{HF}, 1 \mathrm{H}=, 1 \mathrm{H}, 1 \mathrm{HK}, 1 \mathrm{HF}, 1 \mathrm{H}), 1 \mathrm{H}, 1 \mathrm{HK}$,

$21 H I, 1 H), 1 H(, 1 H K, 1 H L, 1 H), 1 H(, 1 H K, 1 H E, 1 H), 1 H(, 1 H K, 1 H N, 1 H), 1 H(, 1 H K$,

$31 \mathrm{HD}, 1 \mathrm{H}) /$

DATA (EOF $(6, J), J=1,28) / 1 H E, 1 H C, 1 H F, 1 H=, 1 H E, 1 H C, 1 H F, 1 H, 1 H, 1 H$,

$21 H, 1 H, 1 H, 1 H, 1 H, 1 H, 1 H, 1 H, 1 H, 1 H, 1 H, 1 H, 1 H, 1 H, 1 H, 1 H$,

$31 \mathrm{1H}, 1 \mathrm{H}$,

DATA (ECIF $(7, J), J=1,28) / 1 H E, 1 H Q, 1 H F, 1 H=, 1 H(, 1 H K, 1 H F, 1 H), 1 H(, 1 H K$,

$21 H I, 1 H), 1 H(, 1 H K, 1 H L, 1 H), 1 H(, 1 H K, 1 H E, 1 H), 1 H(, 1 H K, 1 H N, 1 H), 1 H(, 1 H K$,

$31 \mathrm{HD}, 1 \mathrm{H}) /$

DATA (SOF $(8, J), J=1,28) / 1 \mathrm{HE}, 1 \mathrm{HC}, 1 \mathrm{HF}, 1 \mathrm{H}=, 1 \mathrm{HE}, 1 \mathrm{HC}, 1 \mathrm{HF}, 1 \mathrm{H}, 1 \mathrm{H}, 1 \mathrm{H}$,

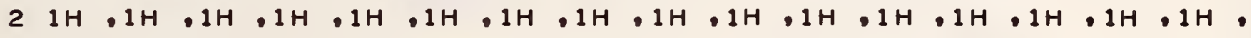

$31 \mathrm{IH}, 1 \mathrm{H}$,

DATA (ECF $(9, J), J=1,28) / 1 H E, 1 H C, 1 H F, 1 H=, 1 H(, 1 H K, 1 H F, 1 H), 1 H(, 1 H K$,

$21 \mathrm{HI}, 1 \mathrm{H}), 1 \mathrm{H}(, 1 \mathrm{HK}, 1 \mathrm{HL}, 1 \mathrm{H}), 1 \mathrm{H}(, 1 \mathrm{HK}, 1 \mathrm{HE}, 1 \mathrm{H}), 1 \mathrm{H}, 1 \mathrm{HK}, 1 \mathrm{HN}, 1 \mathrm{H}), 1 \mathrm{H}, 1 \mathrm{HK}$,

$31 \mathrm{HD}, 1 \mathrm{H}) /$

DATA (EOF $(10, J), J=1,28) / 1 H \equiv, 1 H C, 1 H F, 1 H=, 1 H(, 1 H K, 1 H F, 1 H), 1 H(, 1 H K$,

$21 H I, 1 H), 1 H(, 1 H K, 1 H L, 1 H), 1 H(, 1 H K, 1 H E, 1 H), 1 H(, 1 H K, 1 H N, 1 H), 1 H(, 1 H K$,

$31 H D, 1 H) /$

DATA (DAS $(1, J), J=1,22) / 1 \mathrm{HV}, 1 \mathrm{HI}, 1 \mathrm{HD}, 1 \mathrm{HA}, 1 \mathrm{HR}, 1 \mathrm{H}, 1 \mathrm{H}, 1 \mathrm{H}, 1 \mathrm{HO}, 1 \mathrm{HO}$,

$21 H, 1 H S, 1 H E, 1 H R, 1 H I, 1 H E, 1 H S, 1 H, 1 H, 1 H, 1 H, 1 H$,

DATA (DAS $(2, J), J=1,22) / 1 H, 1 H, 1 H, 1 H, 1 H, 1 H, 1 H, 1 H, 1 H, 1 H$,

$21 \mathrm{1H}, 1 \mathrm{H}, 1 \mathrm{H}, 1 \mathrm{H}, 1 \mathrm{H}, 1 \mathrm{H}, 1 \mathrm{H}, 1 \mathrm{H}, 1 \mathrm{H}, 1 \mathrm{H}, 1 \mathrm{H}, 1 \mathrm{H}$,

DATA (DAS $(3, J), J=1,22) / 1 H V, 1 H I, 1 H D, 1 H A, 1 H R, 1 H, 1 H 5,1 H 4,1 H O, 1 H O$,

$21 \mathrm{H}, 1 \mathrm{HS}, 1 \mathrm{HE}, 1 \mathrm{HR}, 1 \mathrm{HI}, 1 \mathrm{HE}, 1 \mathrm{HS}, 1 \mathrm{H}, 1 \mathrm{H}, 1 \mathrm{H}, 1 \mathrm{H}, 1 \mathrm{H}$,

DATA (DAS( $4, J), J=1,22) / 1 H S, 1 H P, 1 H E, 1 H E, 1 H D, 1 H 2,1 H, 1 H R, 1 H E, 1 H D$,

$21 H U, 1 H C, 1 H E, 1 H D, 1 H, 1 H D, 1 H A, 1 H T, 1 H A, 1 H, 1 H, 1 H$,

DATA (DAS $(5, J), J=1,22) / 1 H V, 1 H I, 1 H D, 1 H A, 1 H R, 1 H, 1 H A, 1 H U, 1 H T, 1 H O$,

$21 \mathrm{HD}, 1 \mathrm{HA}, 1 \mathrm{HT}, 1 \mathrm{HA}, 1 \mathrm{H}, 1 \mathrm{HE}, 1 \mathrm{HI}, 1 \mathrm{HG}, 1 \mathrm{HH}, 1 \mathrm{HT}, 1 \mathrm{H}, 1 \mathrm{H}$,

DATA (DAS $(6, J), J=1,22) / 1 H E, 1 H S, 1 H T, 1 H E, 1 H R, 1 H L, 1 H I, 1 H N, 1 H E, 1 H$,

$21 \mathrm{HA}, 1 \mathrm{HN}, 1 \mathrm{HG}, 1 \mathrm{HU}, 1 \mathrm{HS}, 1 \mathrm{H}, 1 \mathrm{HP}, 1 \mathrm{HD}, 1 \mathrm{H} 2,1 \mathrm{HO}, 1 \mathrm{H} 6,1 \mathrm{H} 4 /$

DATA (DAS $(7, J), J=1,22) / 1 H E, 1 H S, 1 H T, 1 H E, 1 H R, 1 H L, 1 H I, 1 H N, 1 H E, 1 H$,

$21 \mathrm{HA}, 1 \mathrm{HN}, 1 \mathrm{HG}, 1 \mathrm{HU}, 1 \mathrm{HS}, 1 \mathrm{H}, 1 \mathrm{HP}, 1 \mathrm{HD}, 1 \mathrm{H} 2,1 \mathrm{HO}, 1 \mathrm{H}, 1 \mathrm{H} 4 /$

DATA (DAS $(8, J), J=1,22) / 1 \mathrm{HV}, 1 \mathrm{HI}, 1 \mathrm{HD}, 1 \mathrm{HA}, 1 \mathrm{HR}, 1 \mathrm{H}, 1 \mathrm{HA}, 1 \mathrm{HU}, 1 \mathrm{HT}, 1 \mathrm{HO}$,

$21 \mathrm{HD}, 1 \mathrm{HA}, 1 \mathrm{HT}, 1 \mathrm{HA}, 1 \mathrm{H}, 1 \mathrm{HN}, 1 \mathrm{HI}, 1 \mathrm{HN}, 1 \mathrm{HE}, 1 \mathrm{H}, 1 \mathrm{H}, 1 \mathrm{H}$,

DATA (DAS $(9, J), J=1,22) / 1 H V, 1 H I, 1 H D, 1 H A, 1 H R, 1 H, 1 H A, 1 H U, 1 H T, 1 H O$,

$21 H D, 1 H A, 1 H T, 1 H A, 1 H, 1 H N, 1 H I, 1 H N, 1 H E, 1 H, 1 H, 1 H$,

DATA (DAS $(10, J), J=1,22) / 1 H D, 1 H C, 1 H R, 1 H I, 1 H C, 1 H, 1 H D, 1 H I, 1 H G, 1 H I$,

$21 H T, 1 H R, 1 H E, 1 H N, 1 H D, 1 H, 1 H 2,1 H 2,1 H 0,1 H, 1 H, 1 H$,

DATA (ICHARS (I), I=1,26), NCHARS / $1 \mathrm{HN}, 1 \mathrm{HA}, 1 \mathrm{HC}, 1 \mathrm{H},, 1 \mathrm{H},, 1 \mathrm{HV}, 1 \mathrm{HR}, 1 \mathrm{HE}$,

$21 \mathrm{HC}, 1 \mathrm{HK}, 1 \mathrm{HD}, 1 \mathrm{HH}, 1 \mathrm{HM}, 1 \mathrm{HS}, 1 \mathrm{H}(, 1 \mathrm{H}), 1 \mathrm{HS}, 1 \mathrm{HK}, 1 \mathrm{HI}, 1 \mathrm{HP}, 1 \mathrm{H}=, 1 \mathrm{HK}, 1 \mathrm{HS}, 1 \mathrm{HF}$,

$31 \mathrm{H}(, 1 \mathrm{H}), 16 /$

DATA (TFORM $(1, J), J=1,6), N F O R M / 1 H 7,1 H T, 1 H R, 1 H A, 1 H C, 1 H K, 1 /$ 


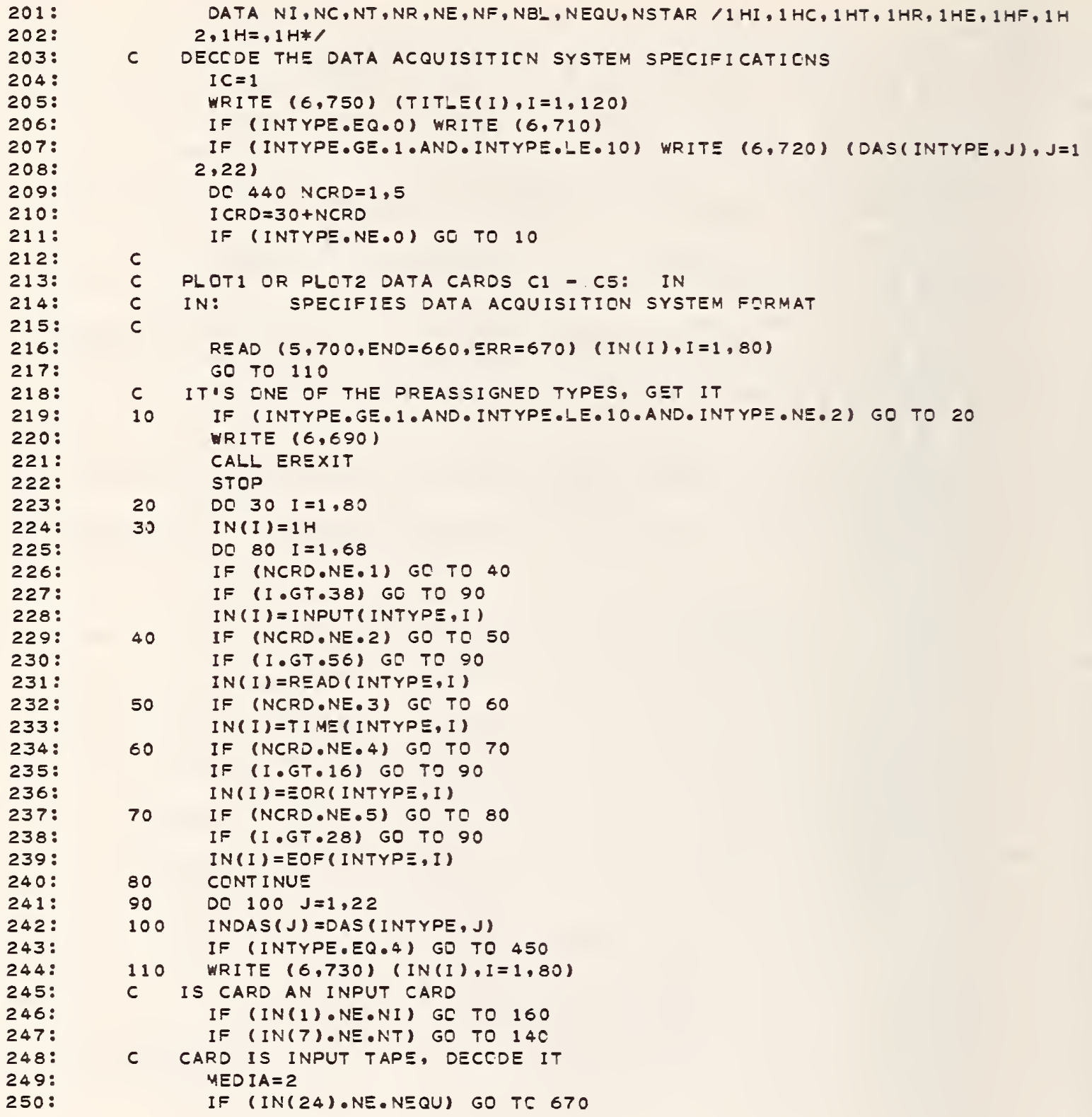




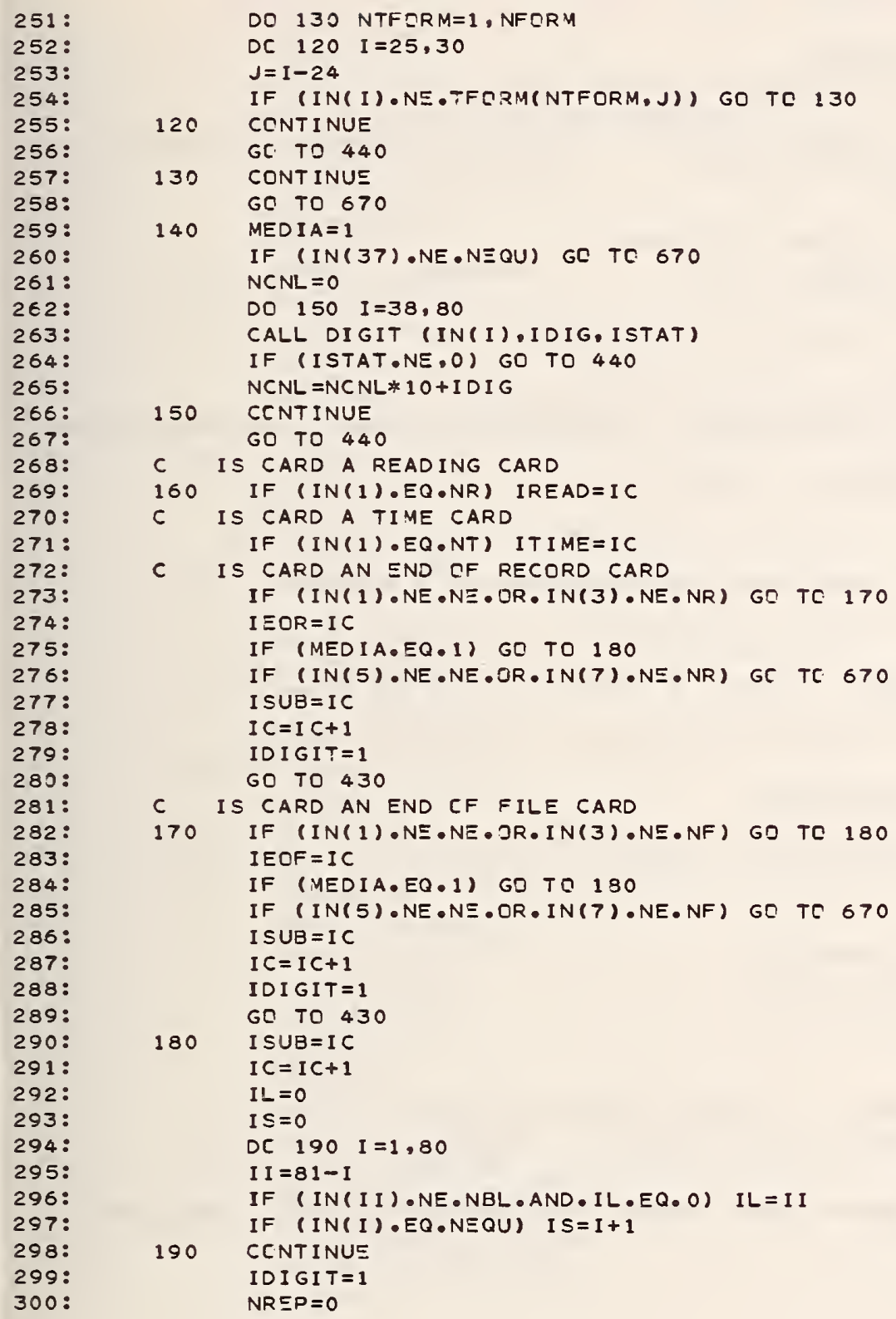




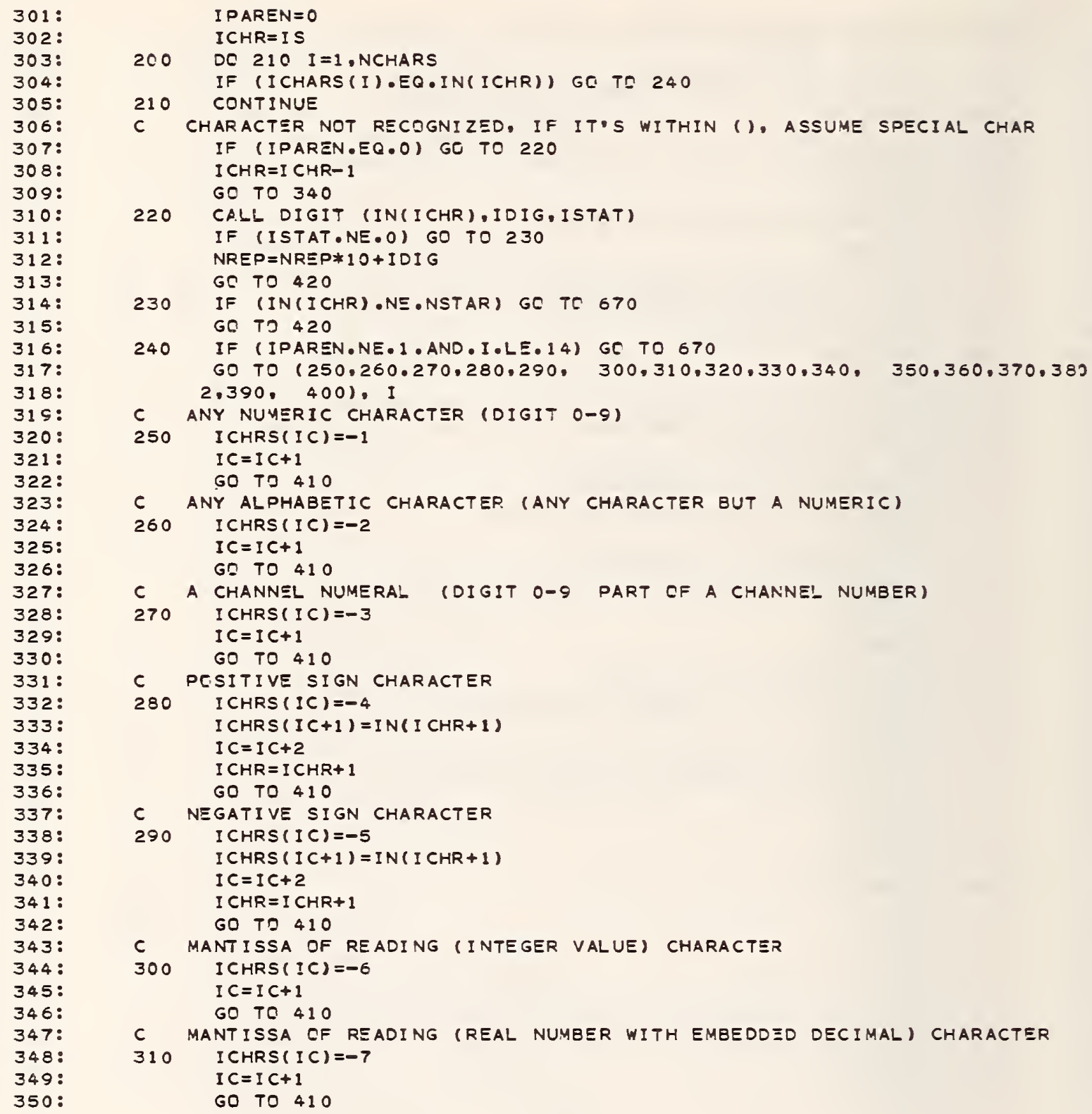




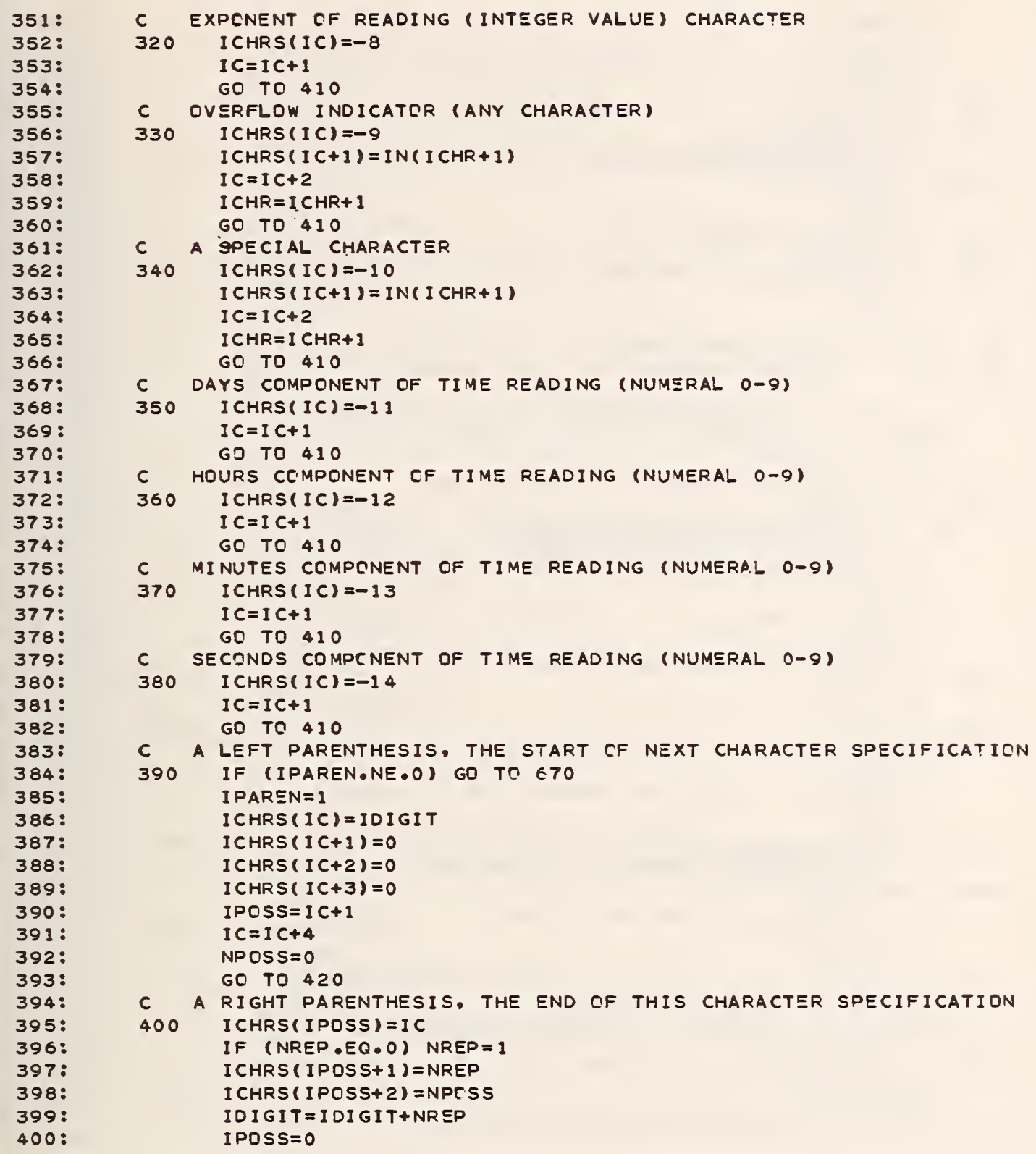




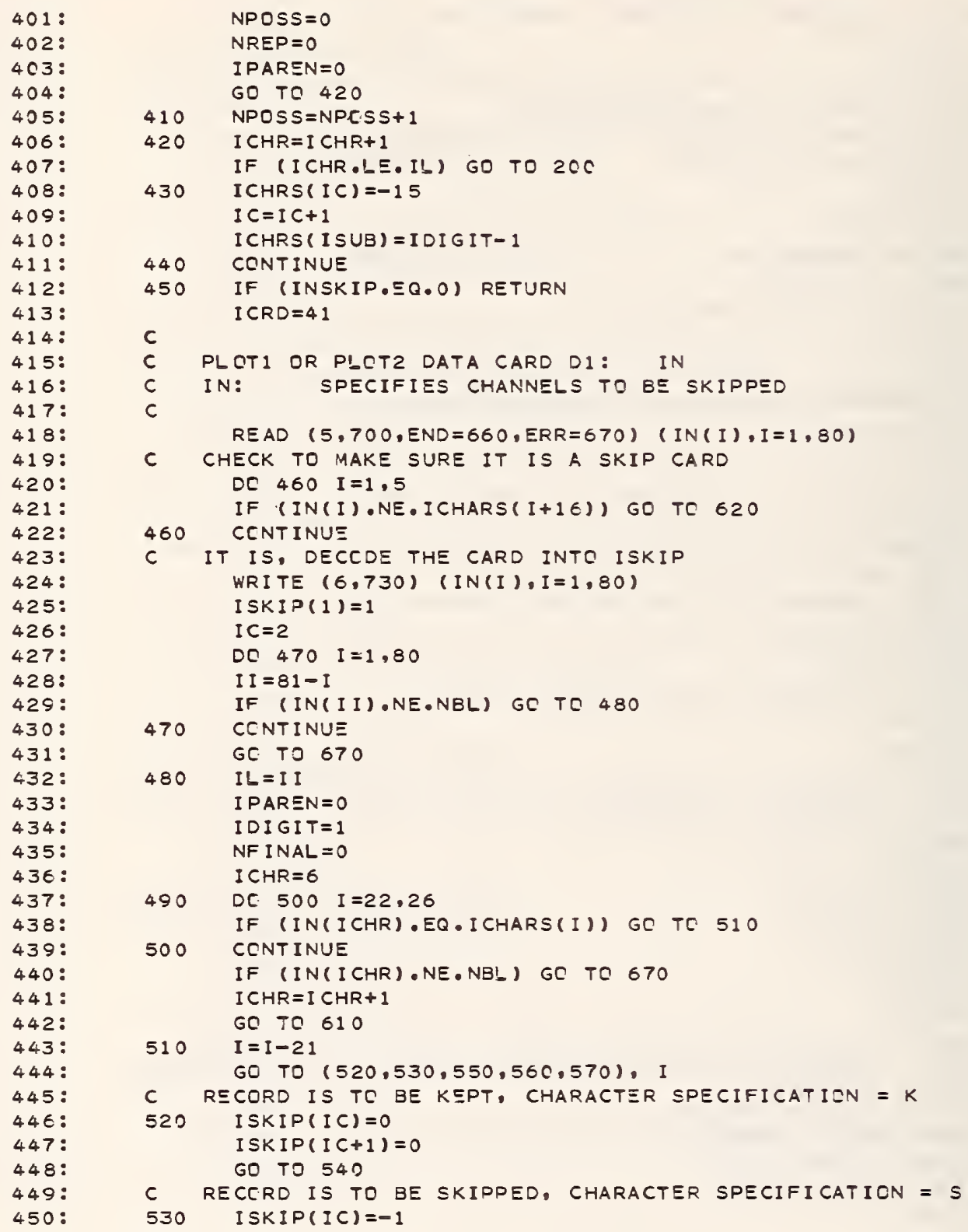




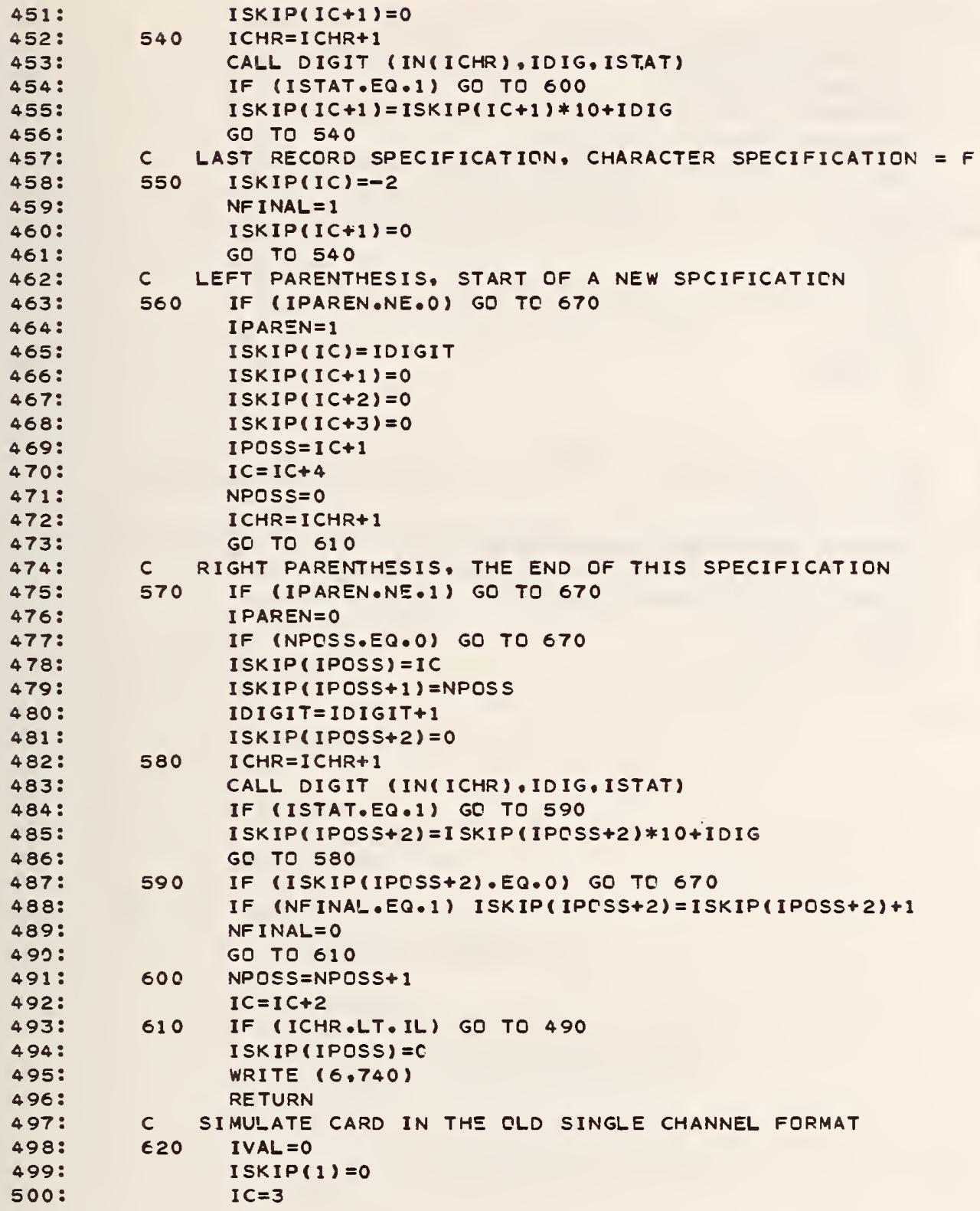




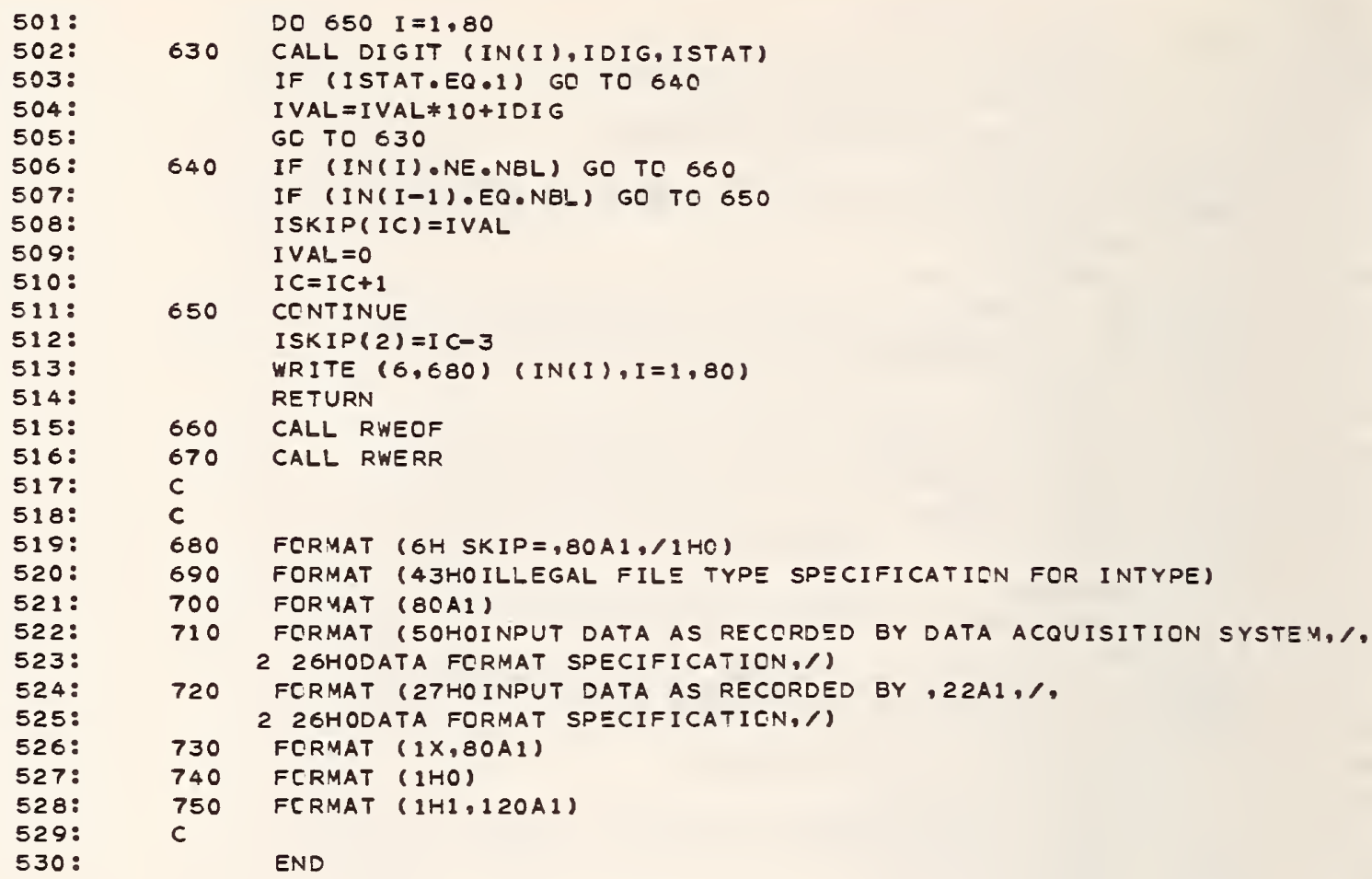


SUBROUT INE DATAIN \&NROW, NCOL,REED, KH, INTYPE, INPRT , INPNCH, MAXR, 2 I I ME, INERR, INSKIP, IOUT, OUTDIM, I CHRS, I NDAS, IREAD, I T I , I EOR, IEOF, 3 MEDIA, TITLE, MCNL, MAXCNL, ISKIP, NCNL, NTFORM)

c

c

COMMON /ERRORS/ ICRD.ISEG

INTEGER OUTDIN,FORMAT, RECHRS

DIMENSION REED (NROW, NCOL), KH(NCOL), IOUT (OUTDIM), MAXR (NCOL).

2 I CHRS ( 320 ), INDAS (22), FORMAT (3), T ITLE ( 120$)$, MCNL (MAXCNL) .

3 ISKIP(320)

DATA NBL,NCOM,NDASH /1H,1H, $1 \mathrm{H}-/$

IF (INTYPE.EQ.4) GO TO 250

IPRT $=0$

I $D$ I $A G=0$

$I E R R=0$

IROW $=0$

IREC $=0$

RECHRS $=0$

10 IRERR $=0$

IRDI $A G=0$

$I=1$

CALL DREAD (IOUT, OUTDIM,INSKIP,L,LCHRS, IREC, IROW, I NTYPE, I CHRS, IREA

2D, IEOR, IEOF, MEDIA, ISKIP, NCNL, NTFORM)

IF (L.LT.O) GO TO 190

DO $20 \mathrm{~J}=1, \mathrm{MAXCNL}$

$M C N L(J)=-1$

20

CONTINUE
$I C N L=0$

$I R=0$

$I B=-I C H R S(I R E A D)$

I $\mathrm{C}=\mathrm{O}$

$I T=0$

$I R O W=I R O W+1$

IF (IROW.LE.NROW) GO TO 30

WRITE $(6.290)$ IROW

CALL EREXIT

C HAS RECORD LENGTH CHANGED IF SC, PRINT A WARNING

30 IF (IROW.EQ.1) RECHRS=LCHRS

IF (RECHRS.EQ.LCHRS) GO TO 40

ID I AG = I D I AG+ I

IRDI $A G=I R D I A G+1$

IF (IERR+IDIAG.GT.INERR. AND.INPRT.NE.-2) GD TO 40

IF (IRERR+IRDIAG.EQ.1) WRITE (6.300)

WRITE $(6,390)$ IREC,RECHRS,LCHRS

RECHRS =LCHRS

C CHECK FOR TIME OR CHANNEL READING

40 CALL MATCH (IOUT, OUTDIM, ICHRS, ITIM, I, ISIGN, I CHAN, IDEC, IVALUE, IEXP, 2IDAY, IHR, IMIN, ISEC, IMAT, NCHRS, NOVFL)

IF (IMAT.EQ.1) GO TO 170 


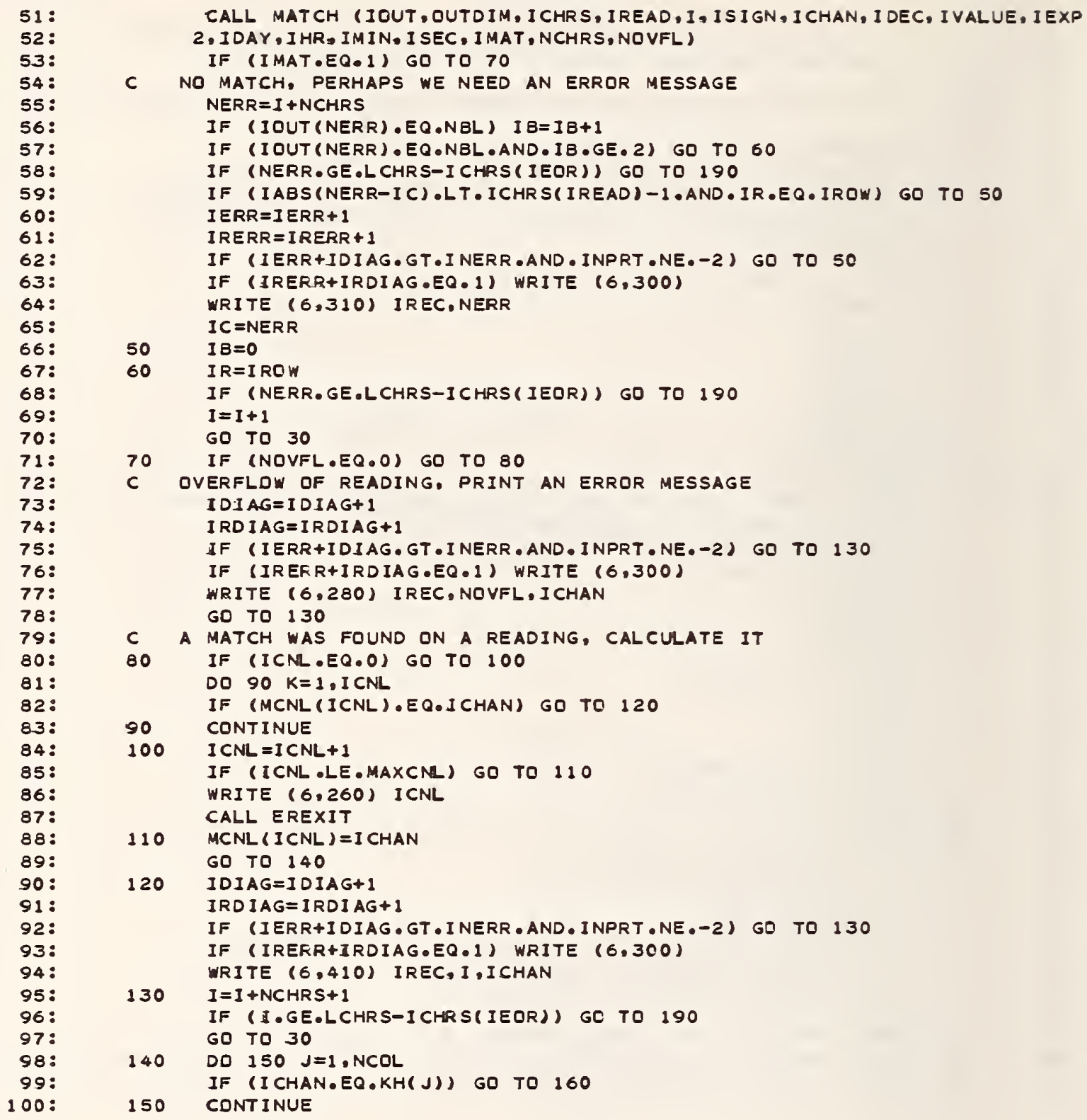

CALL MATCH (IOUT, OUTDIM, ICHRS, IREAD, I, ISI GN, ICHAN, I DEC, I VALUE, IEXP 2, IDAY, I HR, IMIN, I SEC, IMAT, NCHRS, NQVFL) 


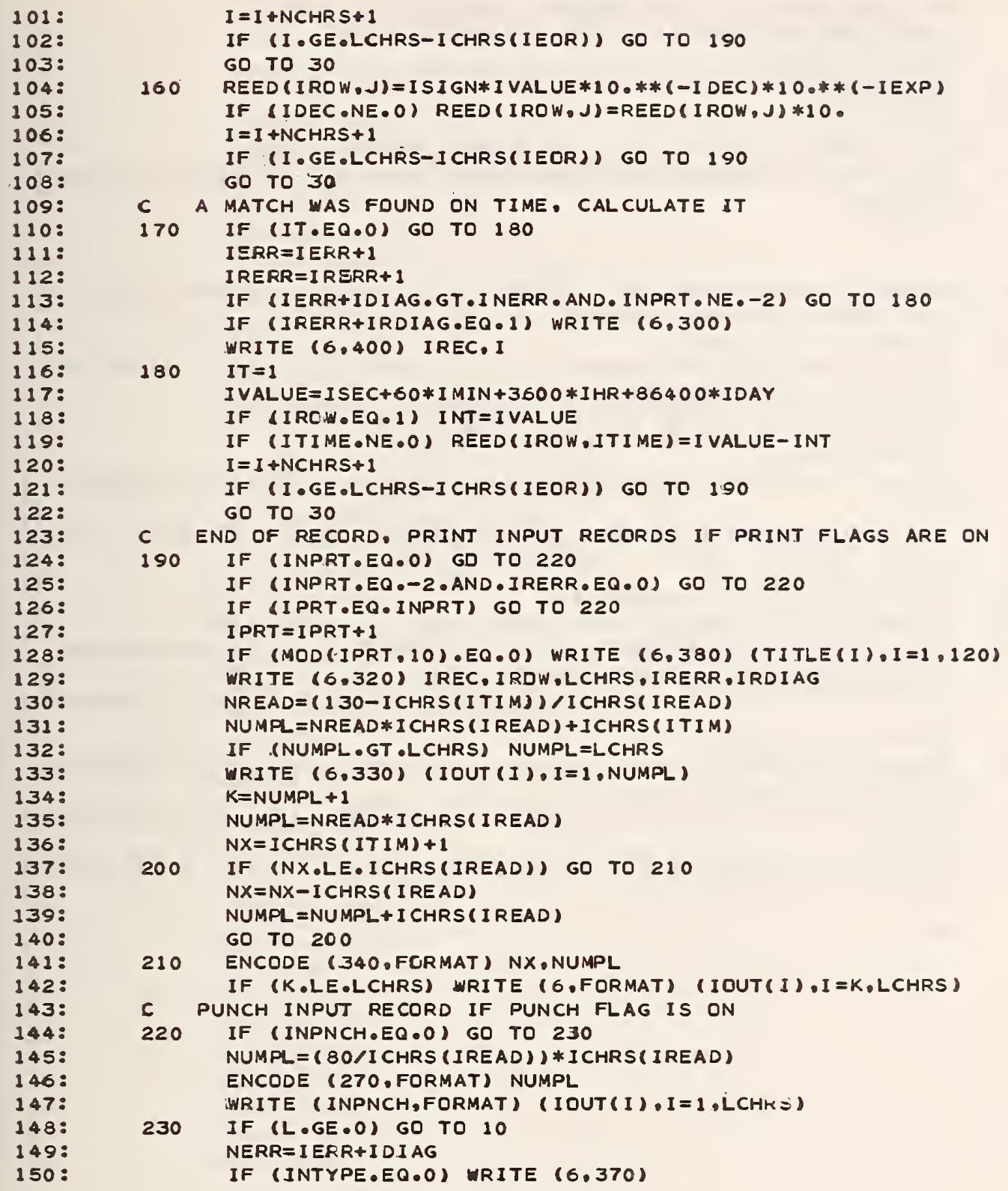




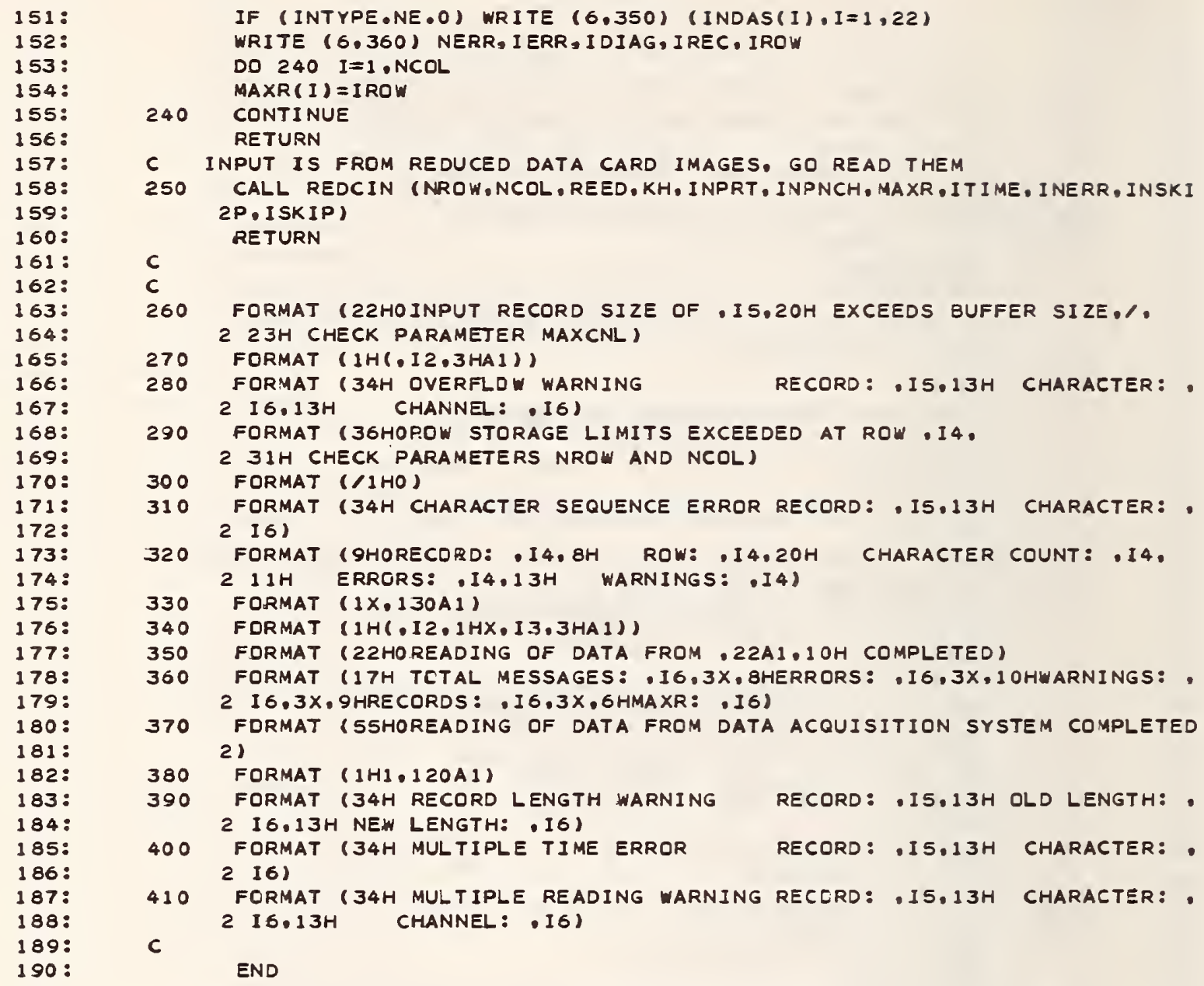


1:

2:

3:

4:

5:

6:

$7:$

8:

9:

10:

11 :

12 :

13:

14:

$15:$

16 :

17:

18:

19:

20:

21:

22:

23:

24:

25:

26:

27:

28 :

29:

30 :

31 :

32:

$33:$

34:

35:

36:

37:

38:

$39:$

40:

41:

42:

43:

44:

45 :

46:

47 :

$48:$

49:

50:

SUBROUT INE DREAD (ICUT, OUTDIM, INSKIP, L, LCHRS, IREC, IRCW, INTYPE,

2 ICHRS, IREAD, IECR, I EOF, MEDIA, ISK IP, NCNL, NTF (RM)

c

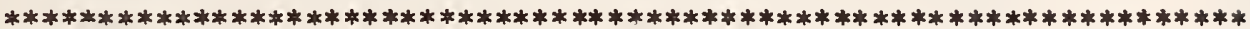

C

INTEGER OUTDIM

CCMMON /ERRORS/ ICRD, ISEG

DI MENSION IOUT (OUTDIM), ISKIP(320), ICHRS(320)

DATA NE,NAT/1HE,1HQ/

$10 \quad I I=0$

IREC $=$ IREC +1

IF (MEDIA.EQ.1) GO TO 80

C INPUT IS TO BE FRDM MAGNETIC TAPE, READ A BLDCK CF DATA IF (NTFORM.NE.1) GQ TO 70

C 7 TRACK TAPE INPUT

CALL NTRAN $(7,26,24,22)$

IOUT ( 1 ) =NE

CALL NTRAN $(7,2$, OUTDIM, IOUT,LSTAT, 20,L)

IF (LSTAT•EQ, -2 ) GO TO 60

IF (IOUT ( 1 ) EQ.NE) LSTAT $=-4$

IF (IOUT(1).EQ.NE) GO TO 160

IF (LSTAT,EQ,-3) CALL NTRAN $(7,22)$

IF (LSTAT.EQ.-3) LSTAT $=L$

IF (LSTAT.LT.O) GO TO 160

IF (L.GT.OUTDIM/6) GO TC 180

DC $30 \quad I=1, L$

$I I=L+1-I$

IC $=$ I OUT ( II)

DO $20 \mathrm{~J}=1,6$

$I K=6 *(I I-1)+7-J$

CALL BYTE (IC,7-J,IOUT(IK))

20 CONTINUE

30 CONTINUE

LCHRS $=6 *$ L

40 IF (IOUT(LCHRS).NE.NAT) GO TO 50 LCHRS $=$ L CHRS -1

GO TO 40

50 IOUT (LCHRS +1$)=1 \mathrm{H}$

IOUT $($ LCHRS +2) $=1$ HX

LCHRS $=\perp C H R S+2$

ICHRS (IEOR) $=2$

GC TO 150

60 IOUT $(1)=1 \mathrm{HF}$

ICUT $(2)=1 \mathrm{HI}$

IOUT $(3)=1 \mathrm{HL}$

I OUT (4) $=1 \mathrm{HE}$

IOUT ( 5$)=1 \mathrm{HN}$

IOUT $(6)=1 \mathrm{HD}$

ICHRS (IEOF) $=6$

GC TO 170 


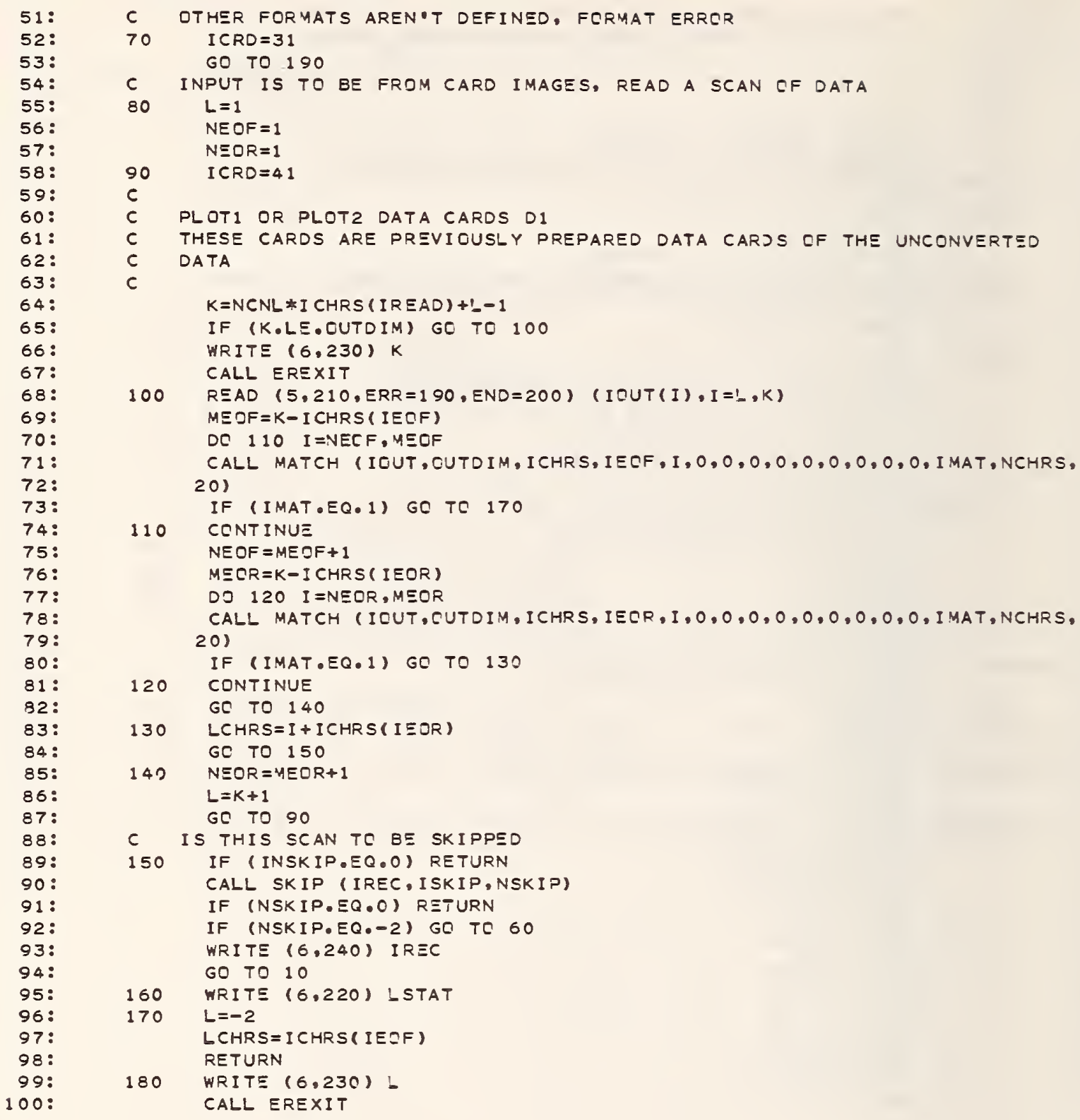




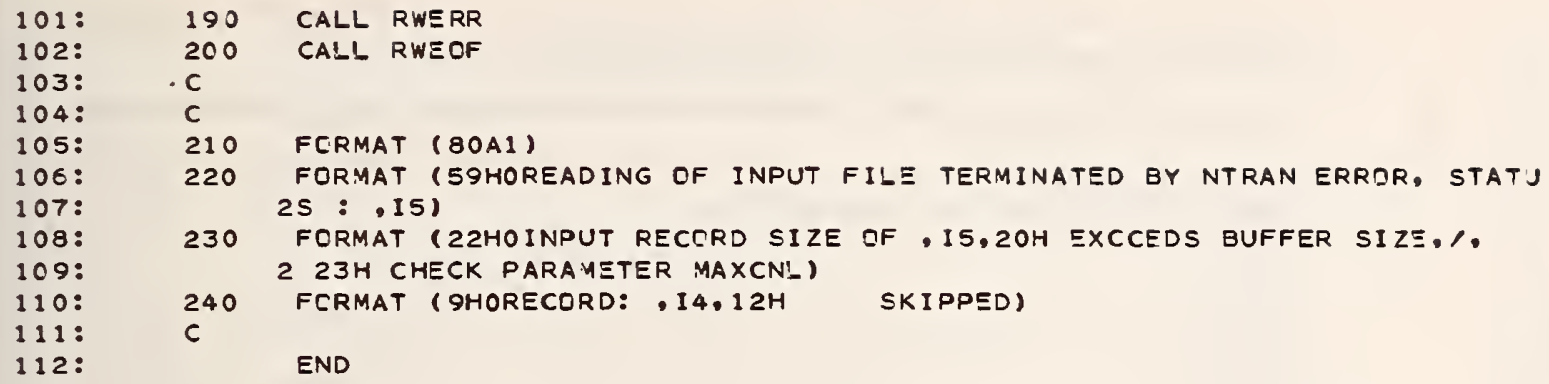


1:

2:

3:

$4:$

$5:$

$6:$

$7:$

8:

$9:$

10:

11 :

12 :

13 :

14:

$15:$

16 :

17:

18:

$19:$

20:

$21:$

22:

23:

24:

25:

26:

$27:$

28:

29:

30:

$31:$

32:

33 :

34:

$35:$

$36:$

37:

38:

39:

40:

41 :

42 :

43:

44:

45:

46 :

$47:$

$48:$

49:

50:

SUBROUTINE MATCH (IOUT, OUTDIM, ICHRS, ISUB, I, ISIGN, I CHAN, IDEC, IVALUE 2, I EXP, IDAY, IHR, I YIN, ISEC, I MAT, NCHRS, NOVF!)

$$
c
$$

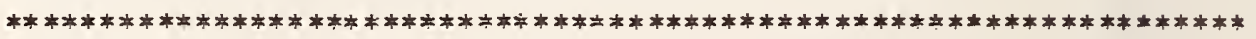




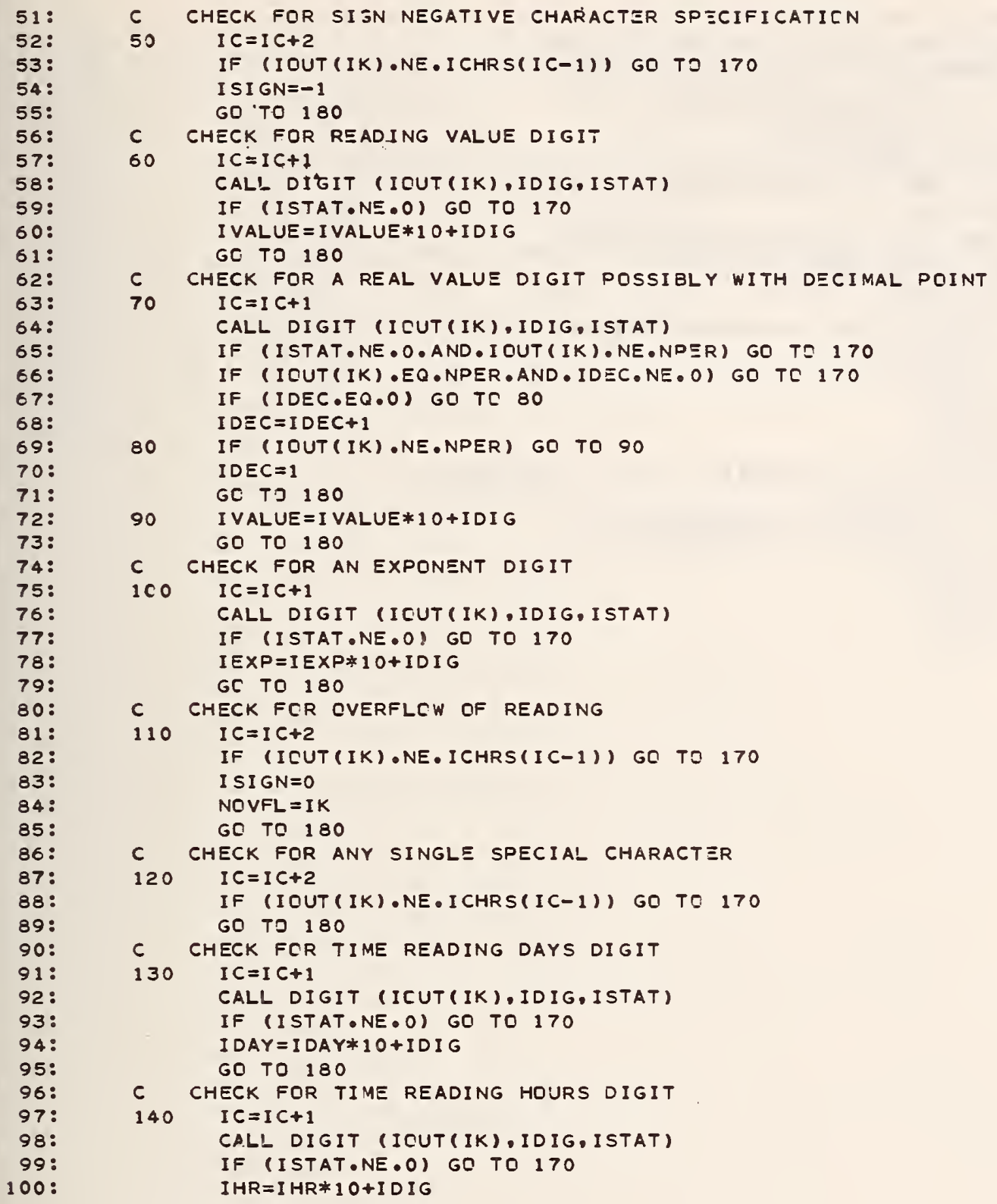




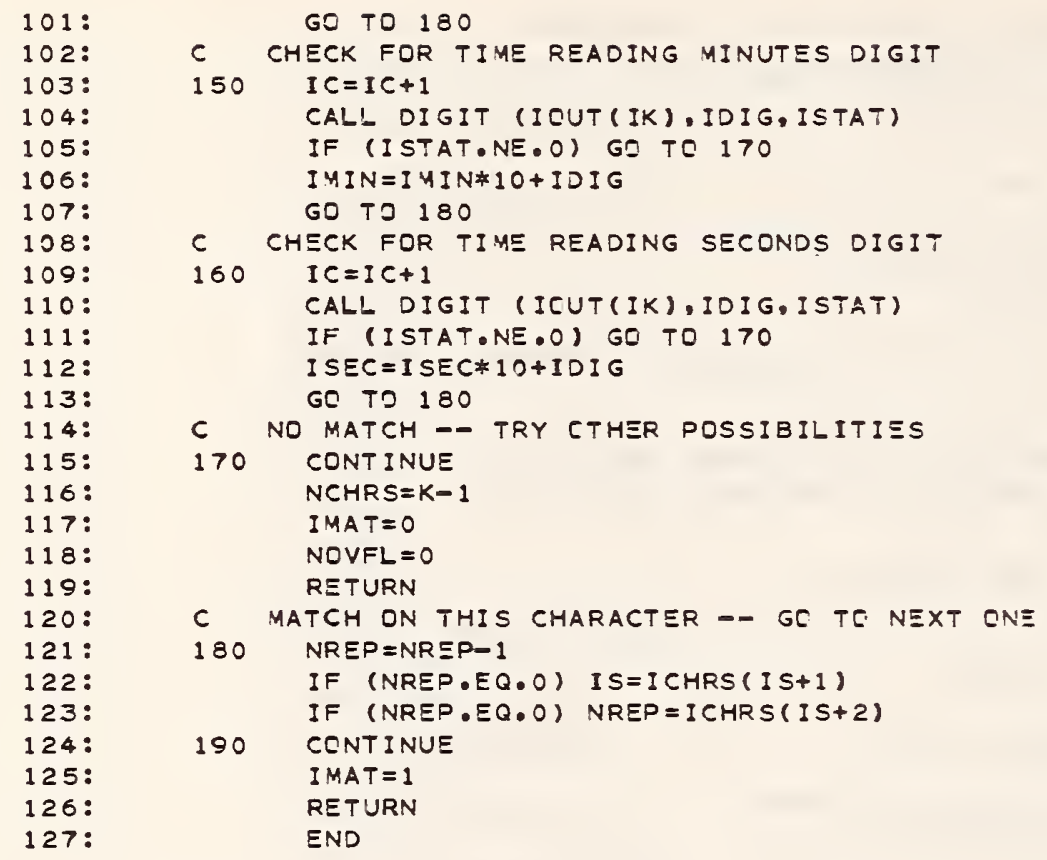




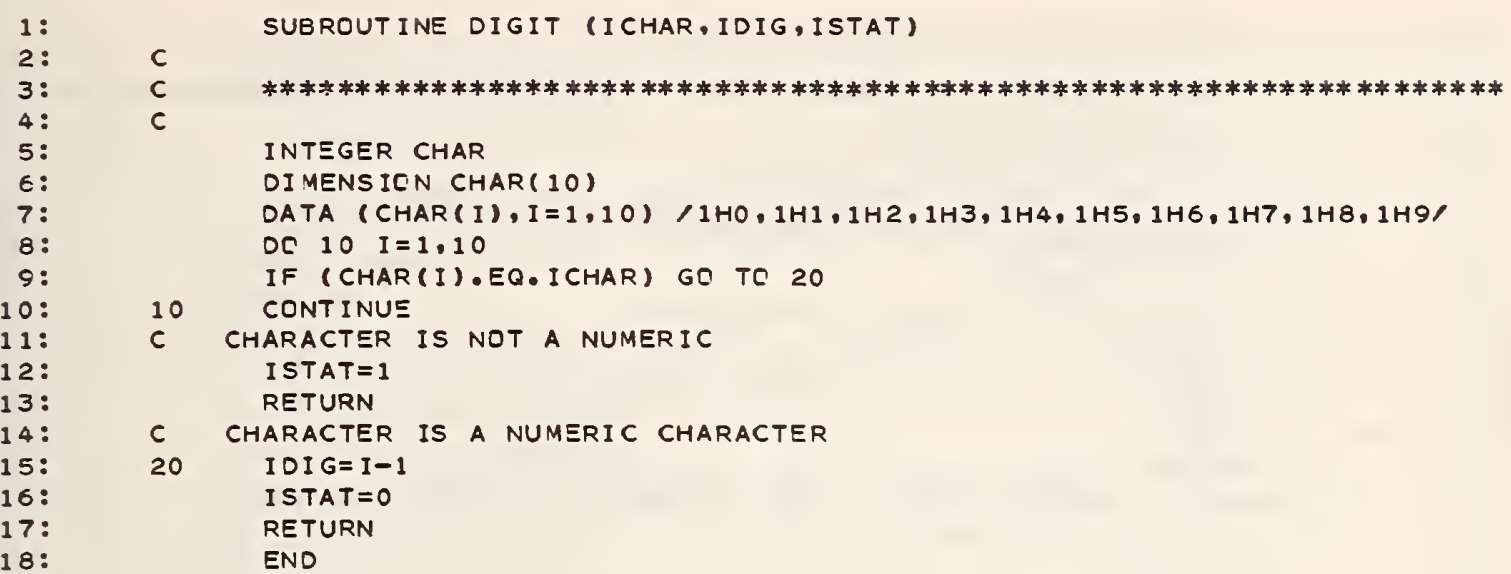




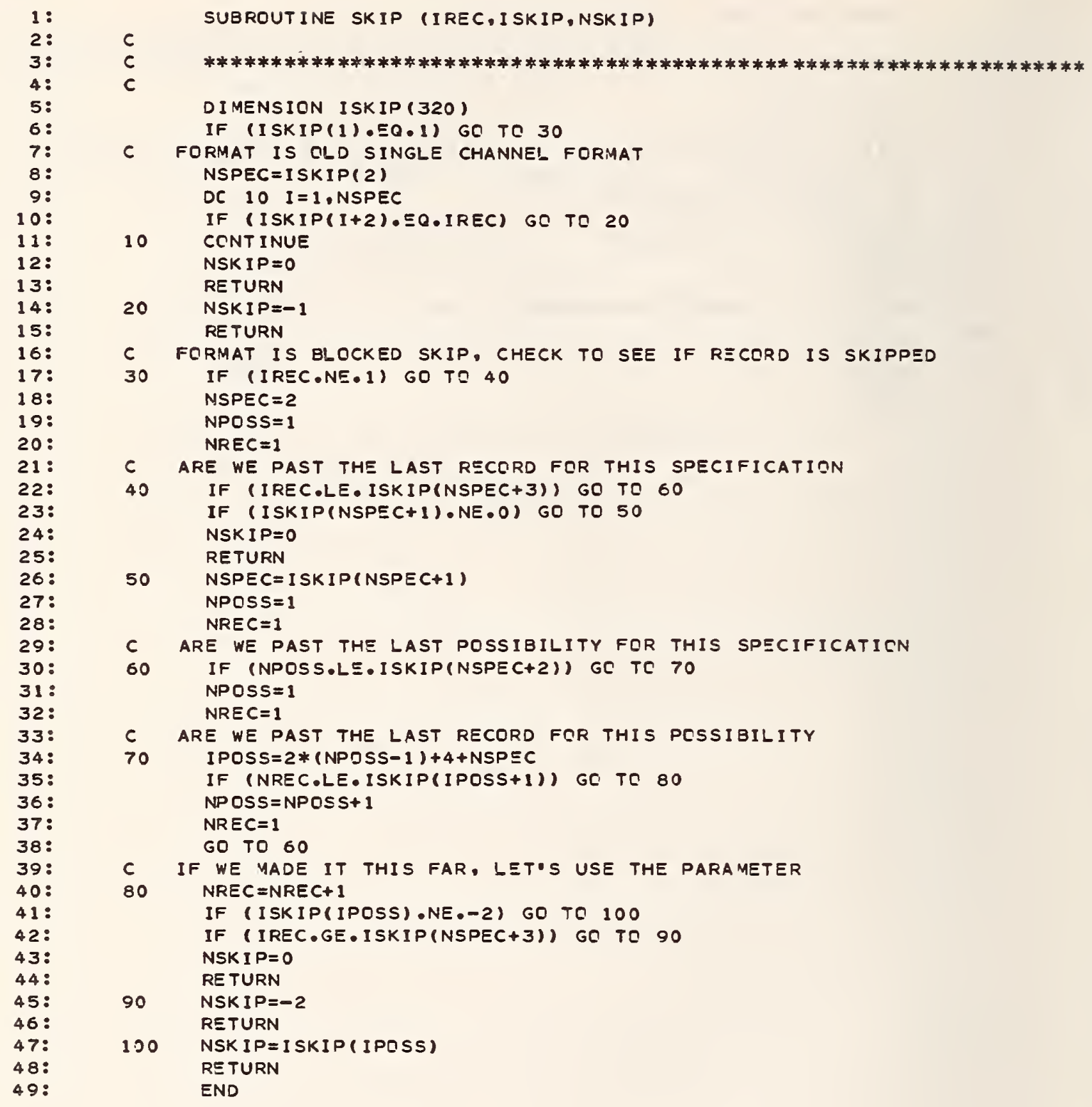


SUBRCUTINE REDCIN (NRCW, NCE:, REED, KH, INPRT, INPNCH, MAXR, IT I ME, INERR 2. INSKIP, ISKIP, LASREC)

C

C

C

CCMMON /ERRORS/ ICRD, ISEG

DI MENSICN REED (NRCW, NCCL), KH(NCOL), YAXR (NCOL), R( 8), IEXP( 8),

2 I SKIP(320)

ICSK I P = O

NCHAN $=0$

C READ CHANNEL NUMBER AND NUMBER OF READINGS

IF (ISEG・EQ.2.CR.ISEG・EQ.3) ICRD $=60$

IF (ISEG.EQ.4) I CRD $=30$

IF (ISEG.EQ.5) I CRD $=32$

$10 \operatorname{READ}(5,190, E R R=120, E N D=130)$ NREAD, I CHAN, IEND

IF (IEND.EQ.999) GC: TC 110

C LCCATE CCLUMN INSTRUMENT IS STCRED IN (IF ANY)

DO $20 \mathrm{~J}=1$. NCOL

IF (ICHAN.EQ.KH(J)) GC TC 30

20 CONTINUE

C THIS CHANNEL IS NOT NEEDED, SKIP CVER THE READINGS NCARDS $=(N R E A D+7) / 8$

READ $(5,140, E R R=120, E N D=130)$ (IN, I =1, NCARDS)

ICSK IP $=$ ICSKIP+1

IF (INPRT.EQ.0) GO TO 10

IF (IPRT,EQ.INPRT) GC TD 10

WRITE $(6,150)$ I CHAN

$I P R T=I P R T+1$

GO TO 10

C READ IN THIS CHANNEI'S READINGS INTO ARRAY REED

30 I $D E C=0$

I RCW $=0$

$40 \operatorname{READ}(5,210, E R R=120, E N D=130) \quad(R(I), \operatorname{IEXP}(I), I=1,8)$

DC $80 \quad I=1,8$

$I R E C=I R E C+1$

IF (INSKIP.EQ.O) GC TO 50

CALL SKIP (IREC, ISKIP,NSKIP)

IF (NSKIP.EQ.-1) GO TC 70

IF (NSKIP.EQ.-2) GO TO 70

50 CONTINUE

IF (IROW.LT.NROW) GO TO 60

WRITE $(6,200)$ I CHAN

CALL EREXIT

STOP

60 IR OW=IRCW+1

REED(IRCW,J)=R(I)*10•**IEXP(I)

70 IF (IREC.GE.NREAD) GO TC 90

80 CONTINUE

GO TD 40

$90 \quad \operatorname{MAXR}(J)=I R C W$ 


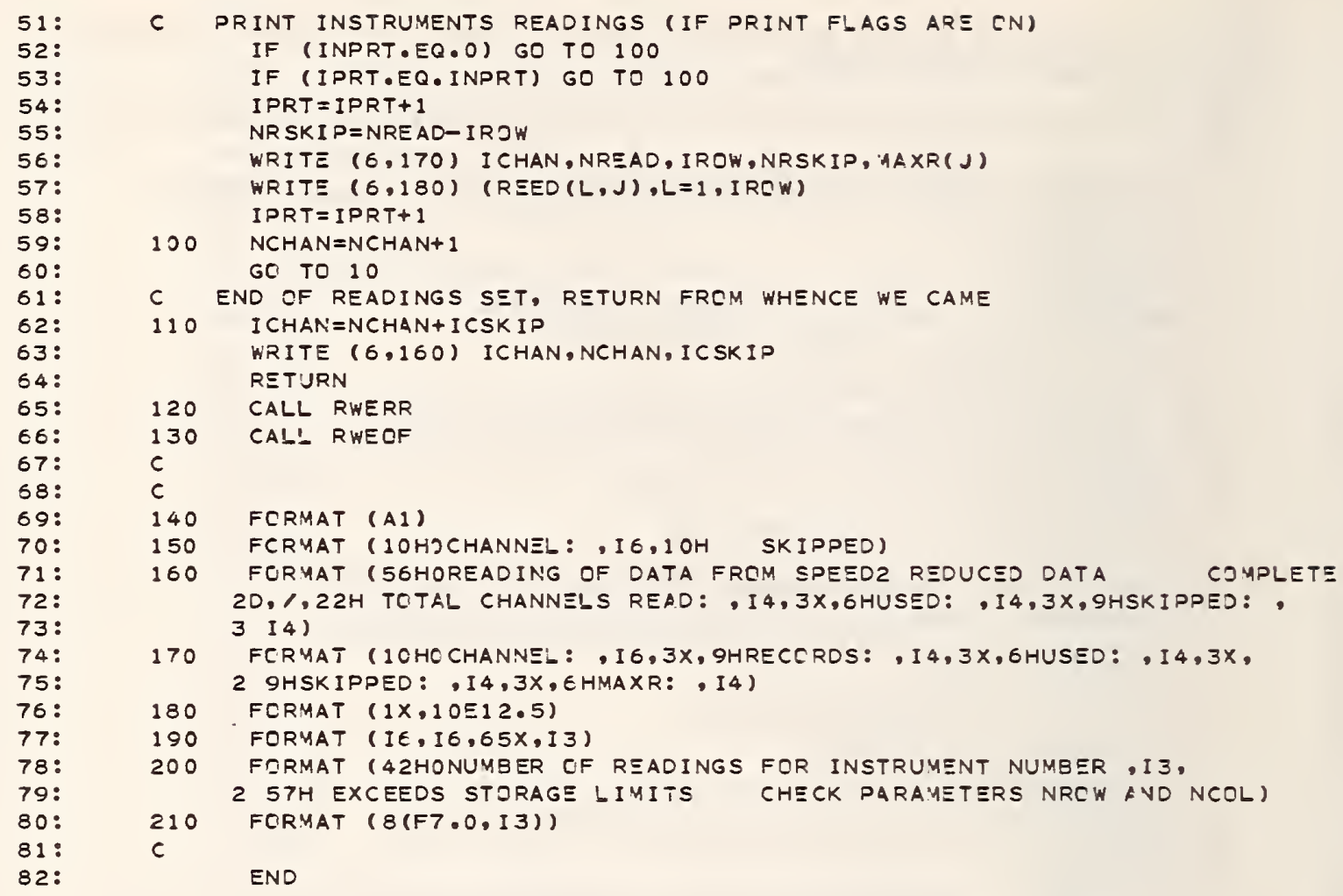


SUBROUTINE PRINT (NROW, NCOL, REED, KH, ITYPE, NAME, ITIME, MAXR, MAXC, 2 TITLE)

C

c

c

DIMENSION REED(NROW, NCOL), KH(NCOL), NAME(NCOL, 6), MAXR (NCOL),

2 I TYPE (NCCL), IBUF(I 20), JBUF (120), TITLE(120)

DATA IB! $/ 1 \mathrm{H} \%$

KHL $=1$

$10 \quad K H H=K H L+9$

$K H H=M I N O(K H H, M A X C)$

$I L=0$

DO $60 L=1$, NROW

IP $=0$

DC $20 \quad I=1,120$

$I B U F(I)=I B L$

$J B U F(I)=I B L$

20 CONTINUE

ENCODE $(70, J B U F)$ (REED $(L, I), I=K H,, K H H)$

DECODE ( $80, J B U F)$ (IBUF (I), I=1,120)

DC $50 \mathrm{I}=\mathrm{KHL}, \mathrm{KHH}$

IF (L.GT.MAXR(I)) GO TO 30

I $P=1$

GO TO 50

30 I WL $=(I-K H L) * 12+1$

$I W H=I W L+11$

DO $40 \mathrm{~J}=I W L, I W H$

$40 \quad I B U F(J)=I B L$

50 CCNTINUE

IF (IP.EQ.0) GO TO 60

$I L=I L+1$

IF (MOD(IL,40).EQ,1) WRITE $(6,140)$ (TITLE(I),I=1,120)

IF (MOD(IL,40),EQ.1) WRITE (6,100) ((NAME(I,II), I I=1,6), I =KHL,KHH)

IF $($ YCD (IL,40),EQ.1) WRITE $(6,110)$ (IBL,KH(I),I=KHL,KHH)

IF $(M O D(I L, 10) \cdot \equiv Q .1)$ WRITE $(6,120)$

IF (ITIME,NE.O) WRITE $(6,130)$ REED(L,ITIME), (IBUF(I), I=1,120)

IF (ITIME.EQ.0) WRITE $(6.90)$ (IBUF $(I), I=1,120$ )

60 CONTINUE

$K H L=K H L+10$

IF (KHL.LE.MAXC) GC TC 10

RETURN

c

70 FCRMAT $(10(1 \times, 610.4,1 \times))$

80 FCRMAT (12CA1)

90 FCRMAT $(1 \times, T 12,120 A 1)$

100 FORMAT (1HO,T12,10(4X,6A1,2X))

110

120

FORMAT $(11 X, 10(A 1,2 X, 1 H(, 16,1 H), 1 X))$

FCRMAT (1H)

FORMAT ( 1 X,G10,4,T12,120A1) 
51: 140 FCRMAT (1H1,120A1)

52:

$53:$

$c$

END 
1:

2:

3:

4:

5:

6:

$7:$

8:

$9:$

10:

11:

12 :

13:

14:

15:

16:

17:

18:

19:

20:

21:

22:

23:

24:

25:

26:

27:

28:

29:

30 :

31:

32 :

33:

34:

35 :

36:

37:

38:

39:

40:

41:

42:

43:

44:

45:

46 :

47:

48:

49:

$50:$

SUBROUT INE PUNCH (NROW,NCCL,REED, KH,NAME, MAXR, MAXC, NTEST, NPNCH)

C

C

C

DI MENSICN REED (NROW, NCOL), KH (NCCL), NAME(NCEL,6), MAXR(NCCL),

2 I BUF ( 80$)$, IZERC (10), ICHR (17)

DATA (IZERO (I), I=1,10), (ICHR(I),I =1,17), IBL / IH+, 1H, ,5*1HO,1H+,

$22 * 1 \mathrm{HO}, 1 \mathrm{HO}, 1 \mathrm{H1}, 1 \mathrm{H} 2,1 \mathrm{H} 3,1 \mathrm{H} 4,1 \mathrm{H}, 1 \mathrm{H}, 1 \mathrm{H}, 1 \mathrm{H}, 1 \mathrm{H}, 1 \mathrm{H}, 1 \mathrm{H}, 1 \mathrm{H}, 1 \mathrm{H}, 1 \mathrm{H}-$

$3,1 \mathrm{H}, 1 \mathrm{H}, 1 \mathrm{H} /$

DO $80 \quad I=1, M A X C$

$K H N=M O D(K H(I), 1000)+1000 * N T E S T$

WRITE (NPNCH,90) MAXR(I),KHN, (NAME $(I, J), J=1,6)$

$M A X R L=1$

$10 \quad M A X R H=M A X R L+7$

$M A, X R H=M I N O$ ( $M A X R H, M A X R(I)$ )

$D C 20 J=1,80$

$I B U F(J)=I B L$

20

CCINTINUE

DC 70 L = MAXRL, MAXRH

$I W L=(L-M A X R L) * 10+1$

IF (REED(L,I) IEQ.0.0) GO TO 30

IEXP = INT $(A L G G 10(A B S(R E E D(L, I))))+1$

IF (ABS (REED $(L, I)) \cdot L T, 1,0)$ I EXP $=I \subseteq X P-1$

I VAL $=I N T(A B S(100000$ * *REED $(L, I) / 10$ ***IEXP $)+0.5)$

IREED $=1000 * I$ VAL + ABS (IEXP)

I BUF ( I WL +1) =I CHR (14)

I $C=I S I G N(1, I E X P)$

$I B U F(I W L+7)=I C H R(12+I C)$

IC =IF IX(SIGN(1, REED $(L, I)))$

I BUF (I WL ) $=\operatorname{ICHR}(16+I C)$

30

$60 \quad J=1.10$

IF (REED(L,I).EQ.0.0) GO TO 50

IF (J.GT.8.OR.J.EQ.3) GO TO 40

I $C=M O D$ ( I REED, 10)

$K=I W L+10-J$

$\operatorname{IBUF}(K)=I C H R(I C+1)$

40 IREED $=I R E E D / 10$

GC TO $\in O$

50

$K=I W L+J-1$

$I B U F(K)=I Z E R C$ ( J )

60

CCNT I NUE

70

80

CONT INUE

WRITE (NPNCH, 100$)$ (IBUF $(J), J=1,80)$

$M A X R L=M A X R L+8$

IF (MAXRL.LE.MAXR(I)) GO TO 10

CONT INUE

WRITE (NPNCH,110)

END FILE NPNCH

REWIND NPNCH

RETURN 


$\begin{array}{lll}\text { 51: } & C & \\ \text { 52: } & C & \\ \text { 53: } & 90 & \text { FCRMAT }(216,6 \text { A1) } \\ \text { 54: } & 100 & \text { FCRMAT }(80 A 1) \\ \text { 55: } & 110 & \text { FCRMAT }(T 78,3 \text { H999) } \\ \text { 56: } & C & \\ 57: & & \text { END }\end{array}$




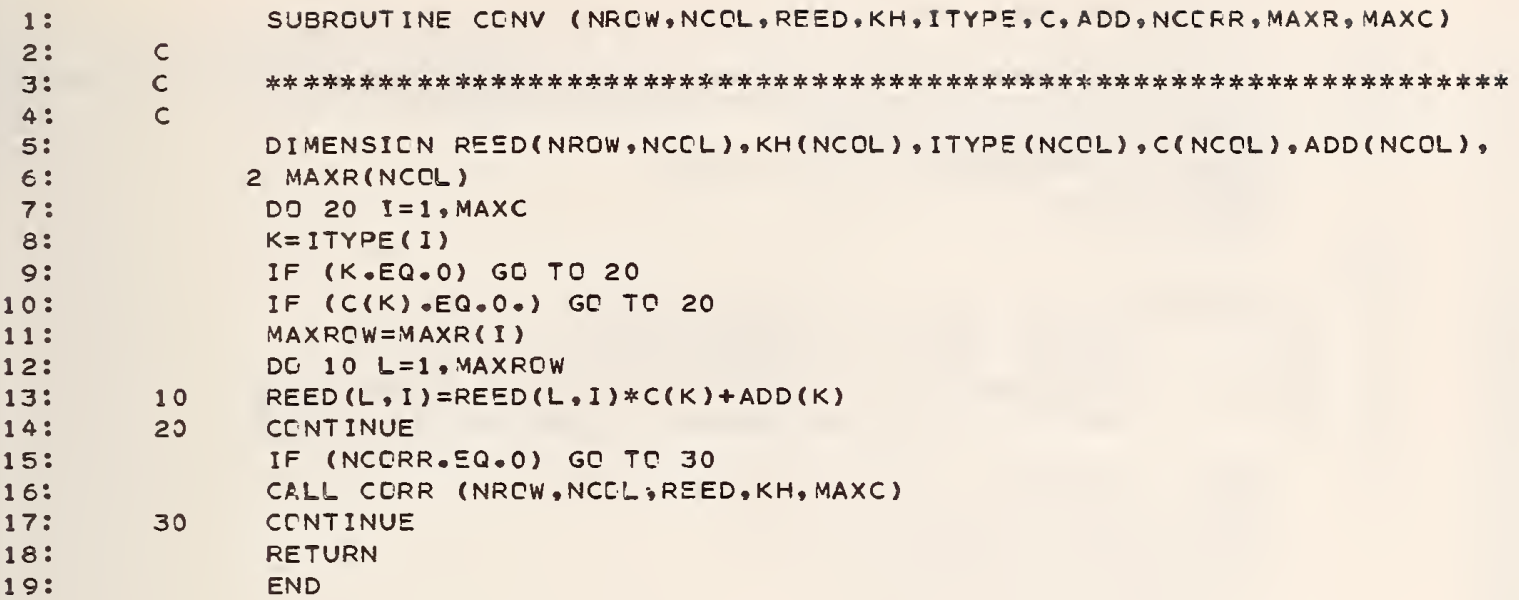




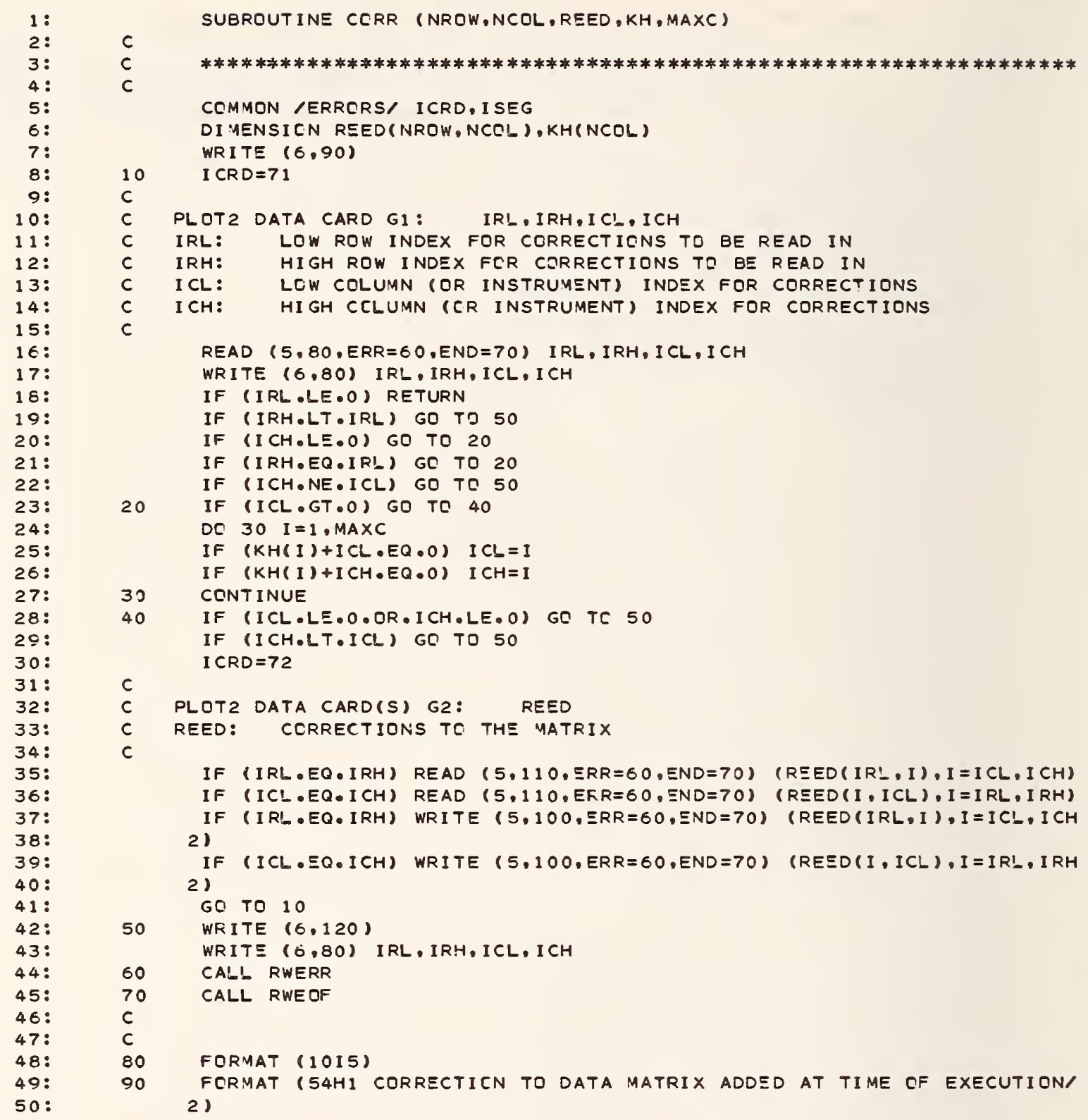




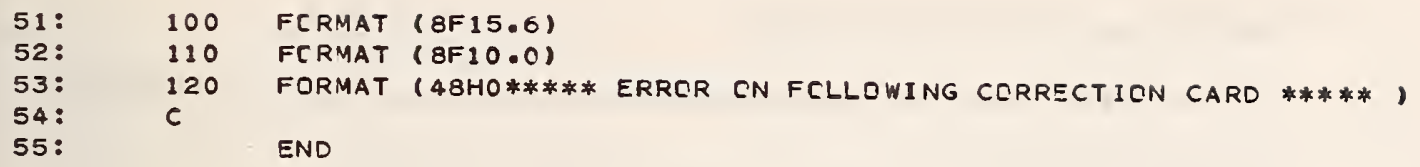




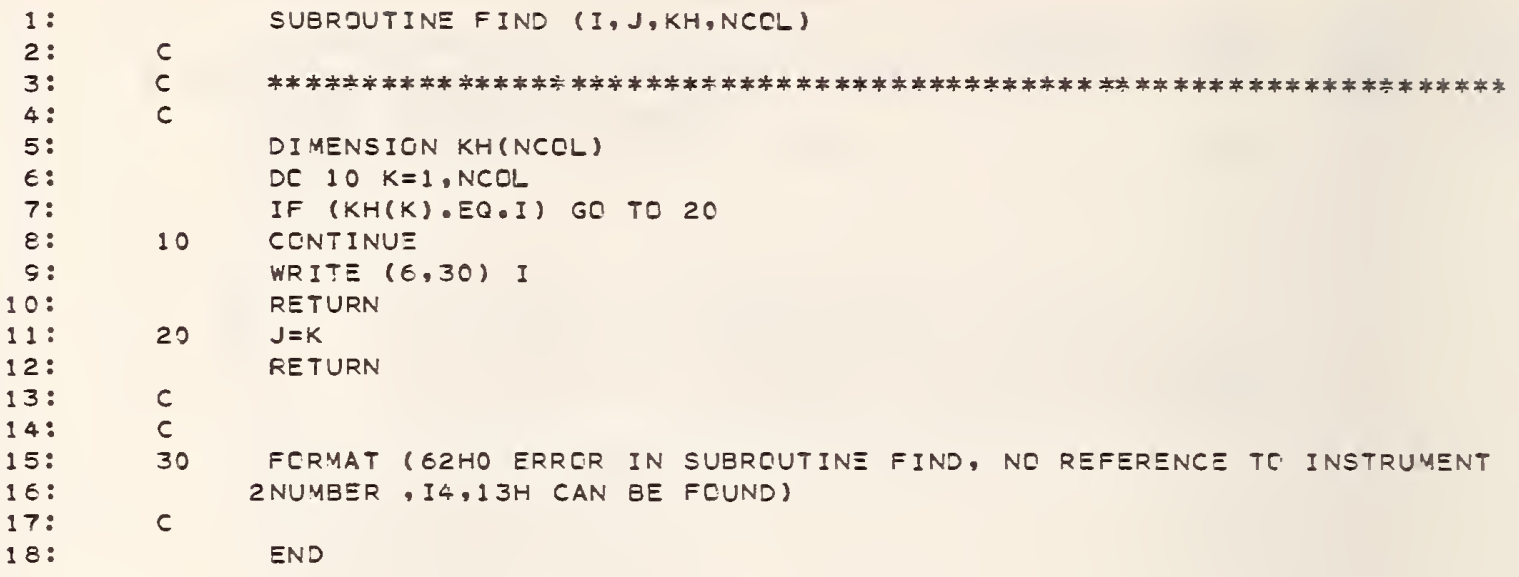


SUBRDUTINE DRAW (JM,IM,X,Y,XO,XM,YO,YM,I IBUFF, JBUFF, IHEAD,TITLE, IPN 2.NRCW.PLTDI Y)

C

C

C

INTEGER PLTDIM

DI MENSION X (NRCW,PLTDIM), Y(NROW, PLTDIM), JM(PLTDIM),P(101), YSC (6), 2 XSC(11), ISYM(6), IPN(PLTDIM,6), IHEAD $(80), \operatorname{IBUFF}(40), J B U F F(40)$,

3 TITLE (120)

INTEGER RM1,R, C,P,DASH,PLUS, BLANK

DATA BLANK, DASH,PLUS, (I SYM(I), I=1,6)/1H, $1 \mathrm{H}-, 1 \mathrm{H+}, 1 \mathrm{H*}, 1 \mathrm{HX}, 1 \mathrm{HO}, 1 \mathrm{H+}$. $21 \mathrm{H}-1 \mathrm{H}, \mathrm{O}$

WR I TE $(6,250)$ (TITLE( I ), I=1,120), (IHEAD(I), I =1,80)

WRITE $(6,280)$

$D Y M=Y M-Y O$

$D X M=X M-X O$

$Y S C(1)=Y M$

$X S C(1)=X 0$

DO $10 L=2,6$

$10 \quad Y S C(L)=Y S C(L-1)-D Y M / 5$ $D C 20 L=2.11$

$20 \quad X S C(L)=X S C(L-1)+D \times M / 10$

DO $30 \mathrm{~J}=1, I M$

$I M I=J M(J)-1$

DO $30 I=1, I M I$

IP $1=I+1$

$I M M=J M(J)$

DC 30 II = IP I, IMM

IF $(Y(I I, J) \cdot L T \cdot Y(I, J))$ GC TO 30

TEMP $=Y(I, J)$

$Y(I, J)=Y(I I, J)$

$Y(I I, J)=T E M P$

TEMP $=X(I, J)$

$X(I, J)=X(I I, J)$

$X(I I, J)=$ TEMP

30 CONTINUE

$R=0$

$L=0$

$40 \quad R=R+1$

IF (R.LE.6.OR.R.GE.47) GO TO 50

I CHAR $=$ I BUFF $(R-6)$

GC TO 60

50 ICHAR=BLANK

60 CONTINUE

RM $1=R-1$

$A R 1=(R M 1-.5) / 50$.

$A R 2=(R M 1+.5) / 50$.

DO $70 \quad C=1,101$

$70 \quad P(C)=B L A N K$

$J=I$ Y 


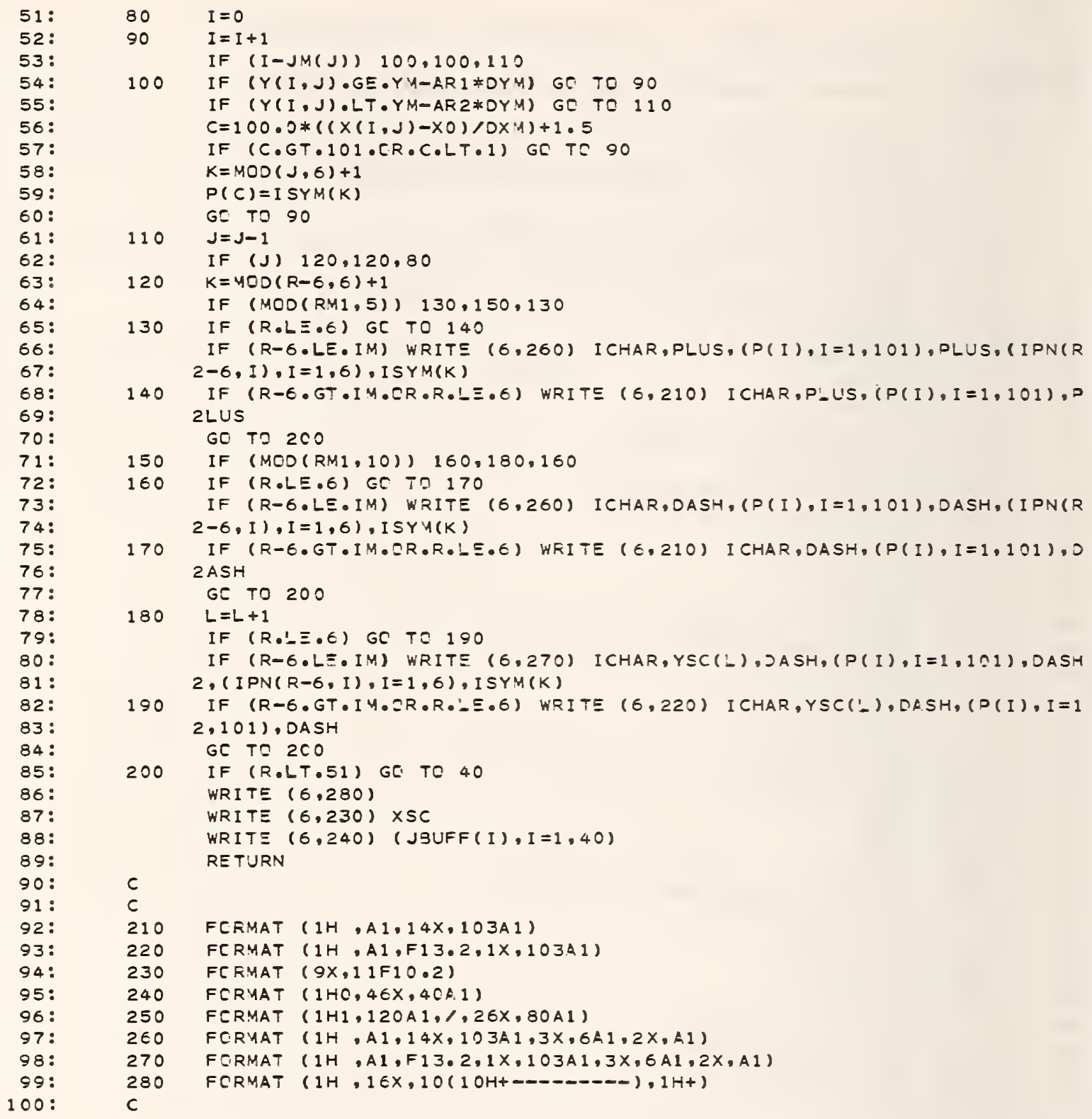




1:
2:
$3:$
$4:$
$5:$
$6:$
$7:$
$8:$
$9:$
$10:$
$11:$
$12:$

SUBROUTINE TEXT (XPOS, YPCS, HEIGHT, ICHARS, ANGLE, NCHRS)

$c$

c

c

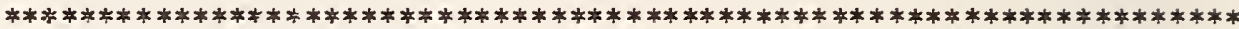
DIMENSION ICHARS (NCHRS)

DO $10 \quad I=1$, NCHRS

$X T P C S=(I-1) * H E I G H T * \operatorname{COS}(A N G L \equiv * 0.01745329)+X P C S$ YTPOS $=(I-1) * H E I G H T * S I N($ ANG $I \equiv * 0.01745329)+Y P E S$

CALL SYMBCL (XTPOS, YTPOS, HEIGHT, I CHARS(I), ANGLE, 1 )

10 CONTINUE

RETURN

END 


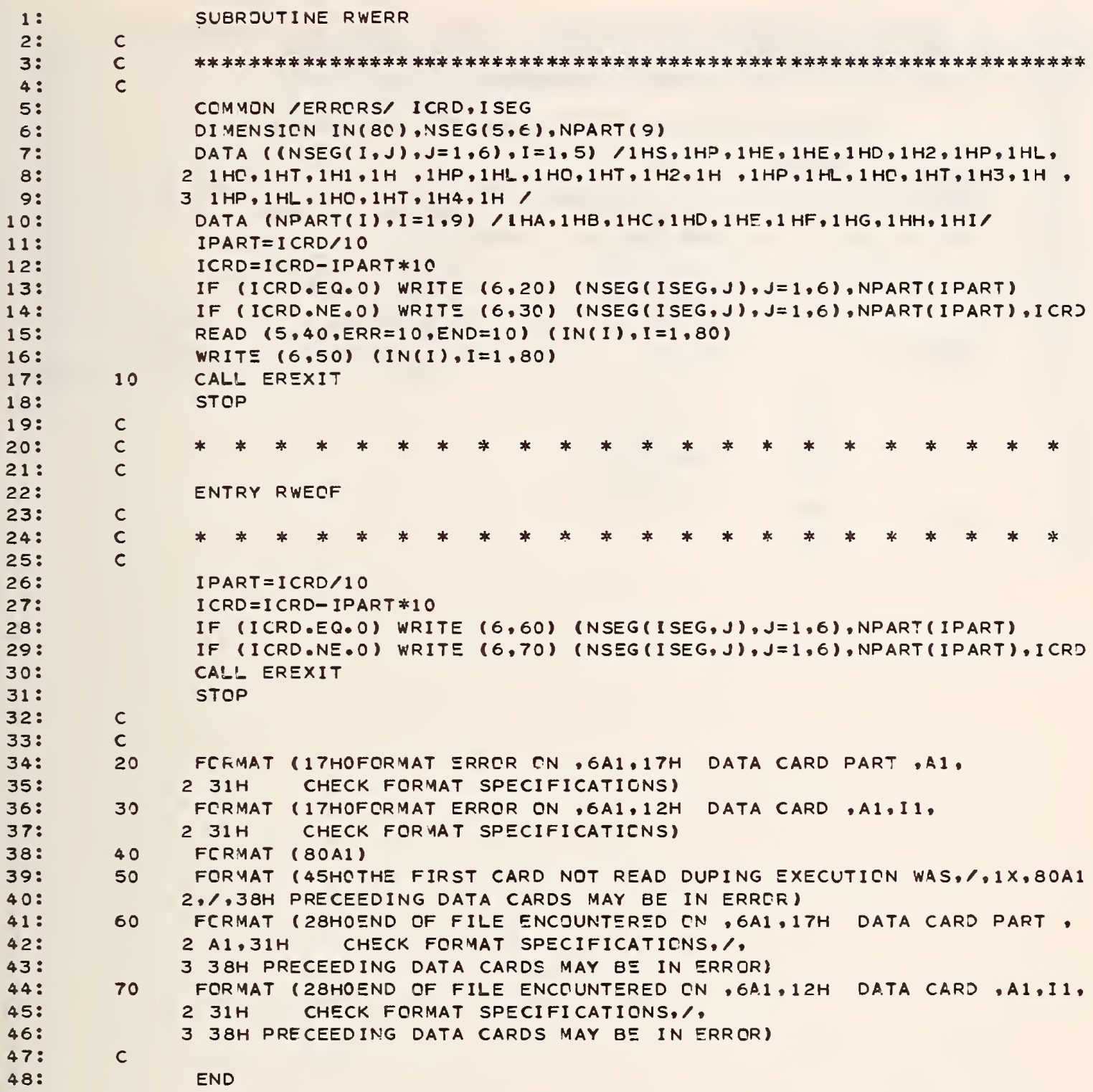




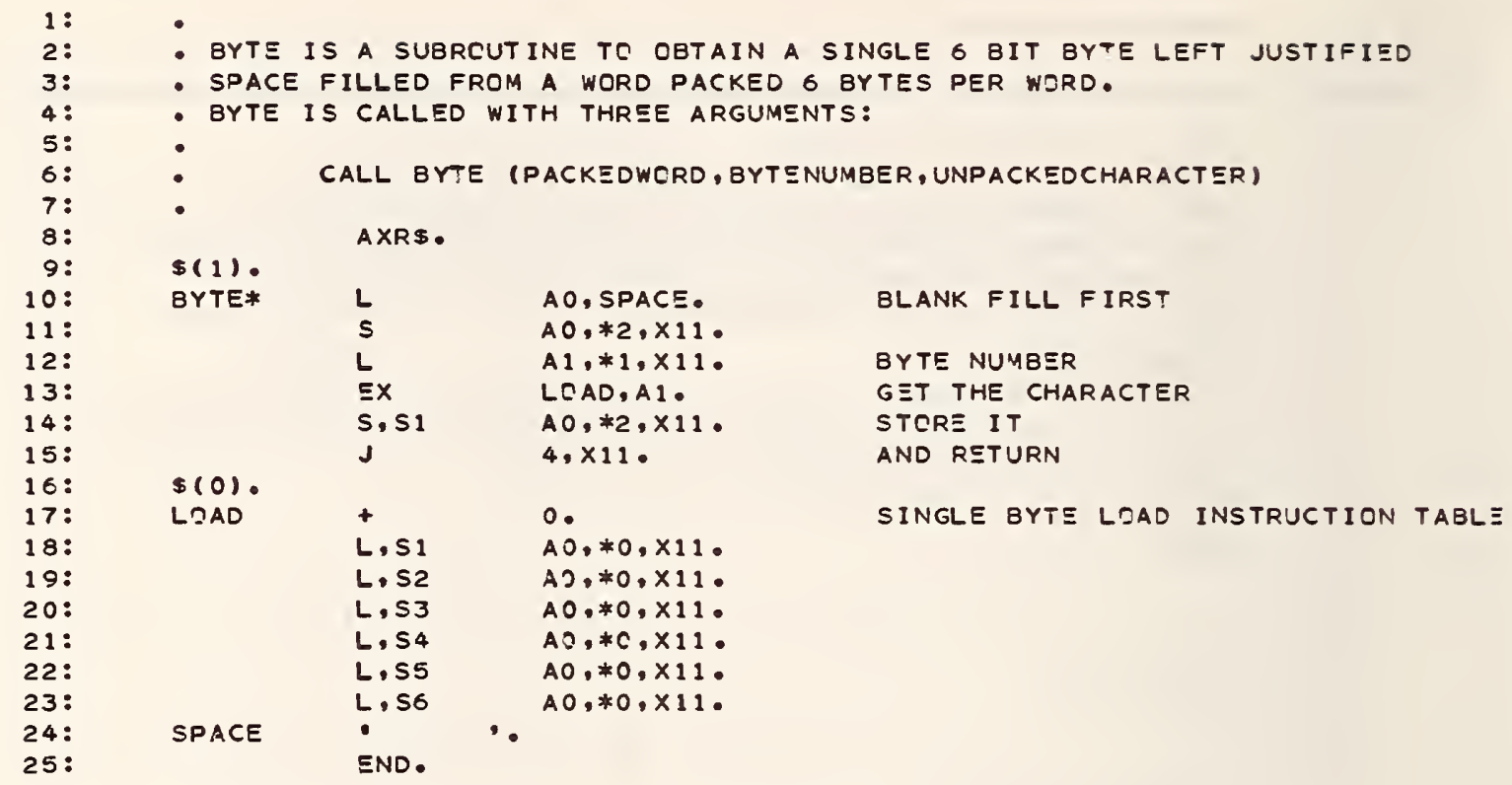


1:
- EREXIT

.

$-$

-

$-$

S(1).

EREXIT*

ER

L.U

ER

ER

$s(0)$.

ERRMSG

LOGMSG
IS A SUGRCUTINE FCR ERROR TERMINATIEN OF THE EXECUTICN OF SPEED2

TO BE USED CNLY ON A UNIVAC 1100 SERIES COMPUTER. A SIMILAR ROUTINE MUST BE WRITTEN FCR CTHER MACHINES TO PRINT AN ERRLR MESSAGE AND BOMB OUT EXECUTION.

AXRS.

- END OF SPEED2 ERRCR EXIT.

- aLCG SPEED2 ERROR EXIT -- RESULTS MAY BE INCCRRECT - •

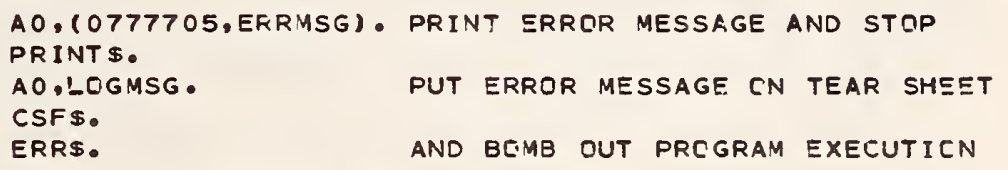




$\begin{array}{ll}\text { 1: } & \text { LIB NBS*CALCOM. } \\ \text { 2: } & \text { LIB NBSHJNTRAN. } \\ 3: & \text { SEG SPEED-2 } \\ \text { 4: } & \text { IN SPEED2 } \\ \text { 5: } & \text { SEG PLCT-1-2*, (SPEED-2) } \\ \text { 6: } & \text { IN PLOT1,PLOT2, ERROR } \\ \text { 7: } & \text { SEG PLOT-3-4*, (SPEED-2) } \\ \text { 8: } & \text { IN PLCT3,PLOT4 } \\ \text { 9: } & \text { END }\end{array}$

- MAIN PREGRAM

- PLIT1, PLCT2

- Plot3 


\section{SAMPLE RUNSTREAMS AND EXAMPLES OF THE USE OF SPEED2}

This appendix provides a number of examples of runstreams, data cards and FORTRAN code used to reduce data collected by data acquisition systems using SPEED2. Examples of the use of PLOT1, PLOT2, PLOT3, and PLOT4 are presented and described in some detail. Several examples are included describing the use of PLOT2, the most complicated phase of SPEED2. 
As a first step in the reduction of data using SPEED2, PLOTl provides the ability to quickly list the data recorded by a data acquisition system, check the correctness of the data and, if desired, output the data to punched cards, mass storage, or magnetic tape. This short example illustrates the use of PLOTl.

Explanation (numbers refer to lines within the example):

1.

Standard UNIVAC 1108 EXEC 8 run initiation card specifying run ID (FIREOI), account number (00000-AAAAAA), project ID (PROJECT), priority (M), and maximum run time and page estimate.

2 .

Assign a temporary mass storage file called 11.

3 . Assign a magnetic tape (FIREOI) containing data recorded by a data acquisition system to logical unit 7 . The assignment options (MTJI), and tape unit type (8C) are dependent upon the data acquisition system tape unit recording format (here, 7 track, 500 BPI, odd parity BCD).

4. Execute the program SPEED2 contained in the mass storage file SPEED2*SPEED2.

5. SPEED2 data card Al -- specifies one data set is to be processed.

6. SPEED2 data card A2 -- specifies PLOTl is to be called.

7. PLOTl data card Al -- entries specify: Esterline Angus PD2064 magnetic tape input (INTYPE=6); a printout of all input data records is to be produced from the data recorded on the magnetic tape (INPRT=-1); a copy of the input data is to be output to logical unit 11 (INPNCH=1l); up to 100 error messages are to be printed (INERR=100); and no input data records are to be skipped -- do not read PLOTl data cards Dl (INSKIP=0).

8-9. PLOTl data cards Bl and B2 -- title of the experiment.

10. Copy the raw data written into the mass storage file called 11 into a permanent mass storage file called FRANKLIN. The data is identified by the element name FIREOl/RAW within the file.

11. UNIVAC 1108 EXEC 8 run termination card. 
1: QRUN, M FIREO 1,00000-AAAAAA, PECJECT, 1.200

2: $\quad$ ASG,T 11.

3: DASG,MTJI 70,8C,FIREO 1

4: \XQT SPEED2*SPEED2.SPEED2

5:

6:

7:

8:

9:

10:

11:

1000

$6 \quad-1 \quad 11 \quad 100 \quad 0$

-FRANK-IN HEATER -- FIREPLACE' FIRE TEST

TEST: FIREO I

DCOPY, I 11 ,FRANKLIN .FIREO 1/RAW

DFIN 
After the data have been processed with PLOTl, the data must be reduced and converted into scientific units for analysis. PLOT2 is used for this purpose. In the first example, data recorded on magnetic tape were processed by PLOTl and output to a mass storage file for permanent storage. This example illustrates the use of PLOT2 to reduce data generated by PLOTl and produce printer plots of the data. The data are to be converted with only linear equations. Hence, there is no need to use FORTRAN coding to convert the data.

\section{Explanation (numbers refer to lines within the example):}

1. Standard UNIVAC 1108 EXEC 8 run initiation card.

2. Copy the mass storage file SPEED2*SPEED2 containing all the subprograms of SPEED2 into the temporary workspace file TPF\$.

3-5. Call the FORTRAN language processor to compile the main program SPEED2, changing the size of the data arrays to NROW $=240$ and NCOL $=25$ to allow up to 24 columns and 240 rows of data (note that one extra column is needed by SPEED2 over and above the number columns needed by the user).

6. Call the aMAP processor (the collector) to collect together the relocatable elements of the subprograms of the SPEED2 system to produce an absolute element for execution.

7. Assign a temporary mass storage file called 10.

8. Assign an existing mass storage file called FRANKLIN.

9. Execute the absolute element SPEED2 created earlier with the CMAP processor.

10-11. SPEED2 data cards $A 1$ and A2 -- specifies one data set (NSETS=1) is to be processed by PLOT2 (IPI=0, IP $2=1$, IP $3=0$, IP $4=0$ ).

12. PLOT2 data card Al -- entries specify: Esterline Angus punched card image format (INTYPE=7); only the first two input data records are to printed (INPRT=2); do not output the raw data (INPNCH=0); do not stop processing after reading the input data (INSTOP=0); do not print any input error messages (INERR=0); and do not skip any input data records -- do not read PLOT2 data card DI (INSKIP=0).

13. PLOT2 data card A2 -- entries specify: a test number of zero (NTEST=0); print the transformed data matrix (NPRT=1); output the transformed data matrix to unit 10 -- in this case, a previously assigned mass storage file ( $\mathrm{NPNCH}=10)$; read PLOT2 data cards part I to generate printer plots (NPLOT=1); and do not read PLOT2 data cards part $G$ to correct readings of the data matrix $(\mathrm{NCORR}=0)$.

14-15. PLOT2 data cards $\mathrm{Bl}$ and $\mathrm{B} 2$-- title of the experiment. 
PLOT2 data cards B3 -- instrument definition cards for instruments numbered $0-18$ and a time channel numbered 999.

36.

PLOT2 data card B4 signals the end of the set of instrument defining cards.

37-39. PLOT2 data cards B5 specifying conversion coefficients for instrument types 1,2 , and 3 .

40.

PLOT2 data card B6 signals the end of the set of coefficient cards.

41.

Element FIREO1/RAW in the mass storage program file FRANKLIN contains raw data images in the format of the data acquisition system prepared by using PLOT1 as in example 1. The @ADD

control statement is used to add into the runstream at this point the entire contents of the element. These are PLOT2 data cards part E.

42-45. PLOT2 data cards I1, I2, I3, and I4, the first of several sets of four cards to generate printer plots from the reduced data. Here, channel 999 is plotted as the $\mathrm{x}$-axis versus channel 1 as the y-axis. The axis limits are $0-5000$ for the $x$-axis and $0-0.25$ for the y-axis. The graph title is INCIDENT RADIANT FLUX ON BACK WALL SURFACE. Axis titles are HEAT FLUX WATTS PER SQUARE CM ( $y$-axis) and ELAPSED TIME IN SECONDS ( $x$-axis).

46-69. Six additional sets of four cards to generate six separate printer plots.

70.

A blank card signals the end of the plot generating cards.

71.

Copy the temporary mass storage file named 10 containing the reduced data output by SPEED2 ( $\mathrm{NPNCH}=10$ ) into permanent storage in the element named FIREOI/REDUCED within the mass storage program file named FRANKLIN.

Standard UNIVAC 1108 EXEC 8 run termination card. 


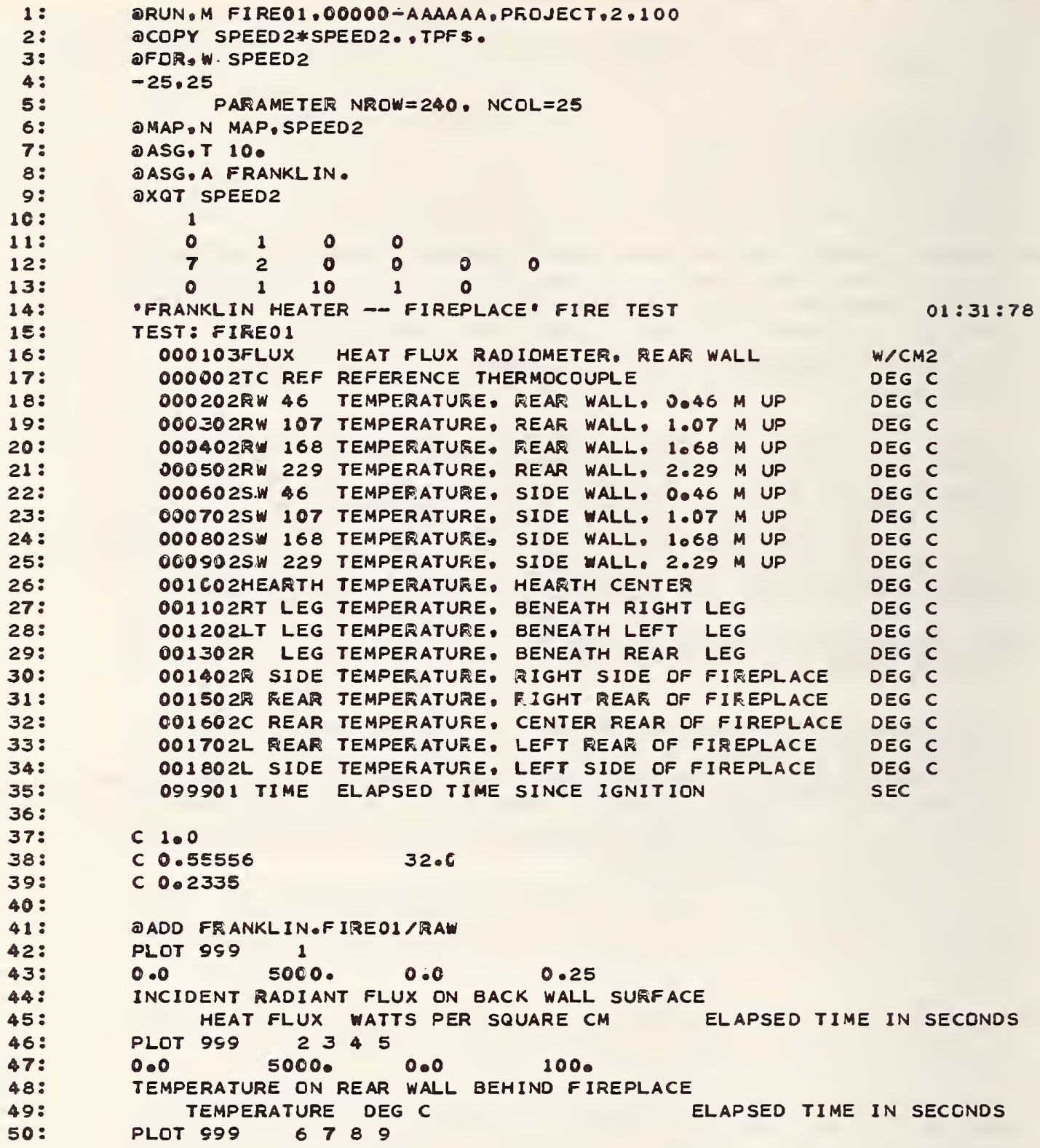




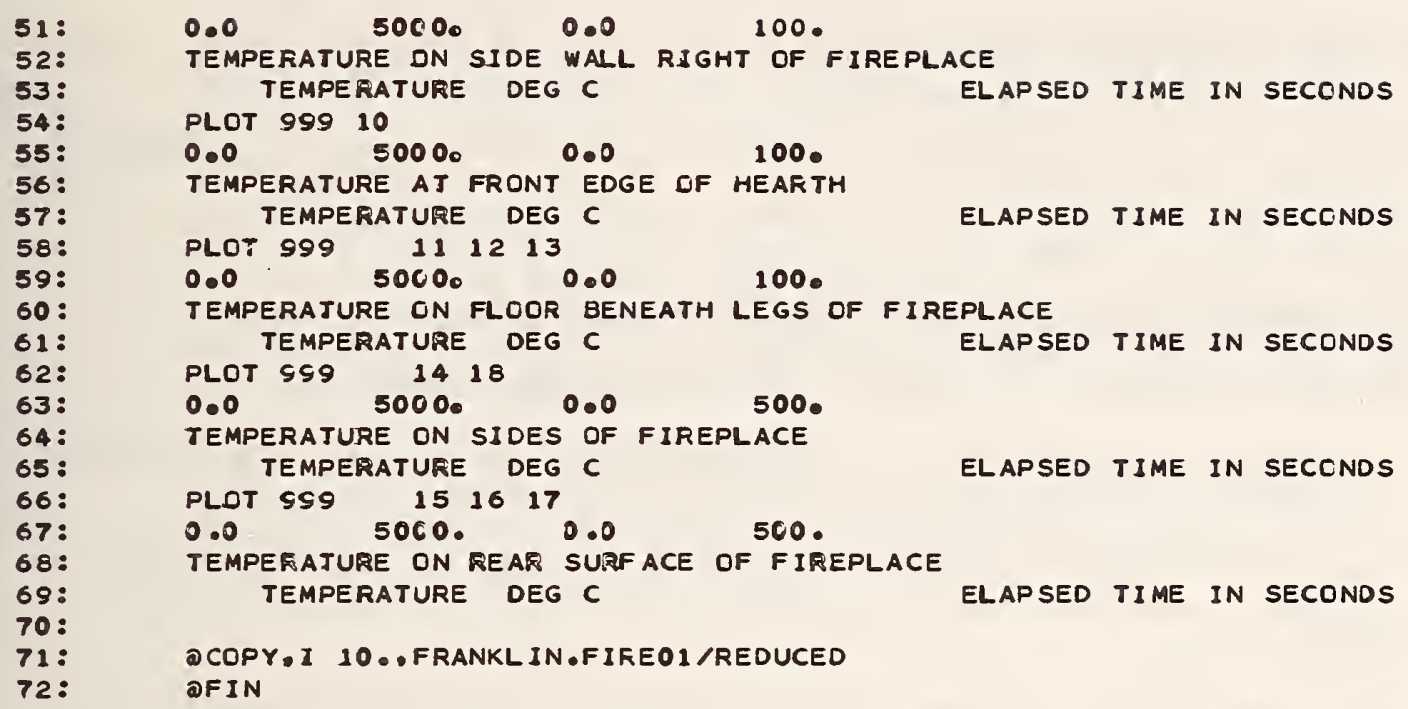


EXAMPLE 3. USING PLOT3 TO GENERATE PEN PLOTS FROM REDUCED DATA

For reports and other presentations of data, the user may desire better quality graphs of the data reduced and stored using PLOTl and PLOT2. In this example, PLOT3 is used to generate a pen plot from data reduced as shown in example 2 .

Explanation (numbers refer to lines within the example):

1 .

2 .

$3-5$.

6.

7 .

$8-9$.

10.

$11-12$

13.

14 .

$15-16$

$17-18$

19.

Standard UNIVAC 1108 EXEC 8 run initiation card.

Copy the mass storage file SPEED2*SPEED2 containing all the subprograms of SPEED2 into the temporary workspace file TPF\$.

Call the FORTRAN language processor to compile the main program SPEED2, changing the size of the data arrays to NROW $=240$ and $\mathrm{NCOL}=3$.

Call the QMAP processor (the collector) to collect together the relocatable elements of the subprograms of the SPEED2 system to produce an absolute element for execution.

Assign an existing mass storage file (A-option) called FRANKLIN. Assign a magnetic tape CAL to logical unit 7 to be used for the output of the CALCOMP plotting commands.

Execute the absolute element SPEED2 created earlier with the @MAP processor.

SPEED2 data cards $A 1$ and $A 2$-- specifies one data set (NSET=1) is to be processed using PLOT3 (IP1, IP2, IP4 equal zero and IP3 equals one).

PLOT3 data card Al -- entries specify: data matrix is not to be printed (NPRT=0); smooth the data before plotting using a running five point linear average (NSMTH=5); ignore all out of bounds points (NOUT=0); place axes on all four sides of each graph produced (NAXIS=1); draw a line through each data point of all curves, placing a special plotting symbol every 10 points (NPNT=10); and do not produce any legends on the graphs (NLEG=0).

PLOT3 data card A2 -- entries specify the size of the graphs produced. The $x$-axis length is 10.0 scaled inches (XSIZE=10.0); the $y$-axis length is 5.0 scaled inches (YSIZE=5.0); and the scaling factor is 0.8 ( $\mathrm{SCALE}=0.8$ ).

PLOT3 data cards Bl and B2 -- the title of the experiment.

PLOT3 data cards B3 -- instrument definition cards for channels 002 and 999.

PLOT3 data card B4 signals the end of the instrument definition cards. 
Element FIREOI/REDUCED in the mass storage program file FRANKLIN contains the set of reduced data output by PLOT2. The GADD control statement is used to add into the runstream at this point the entire contents of the element. These are PLOT3 data cards part $C$.

21-24. PLOT3 data cards D1, D2, D3, and D4 describing the pen plot to be produced. Channel 999 is plotted on the $x$-axis versus channel 002 on the $y$-axis. The axis limits are $0-5000$ for the $x$-axis and 0 - 100 for the y-axis. The graph title is FIGURE 4: SURFACF: TEMPERATURE ON REAR WALL BEHIND FIREPLACE. Axis titles are TEMPERATURE (DEG $C$ ) and TIME SINCE IGNITION (SEC) for the $y$ and $x$ axes respectively.

25. A blank card signals the end of the plot generating cards.

26. Standard UNIVAC 1108 EXEC8 run termination card. 


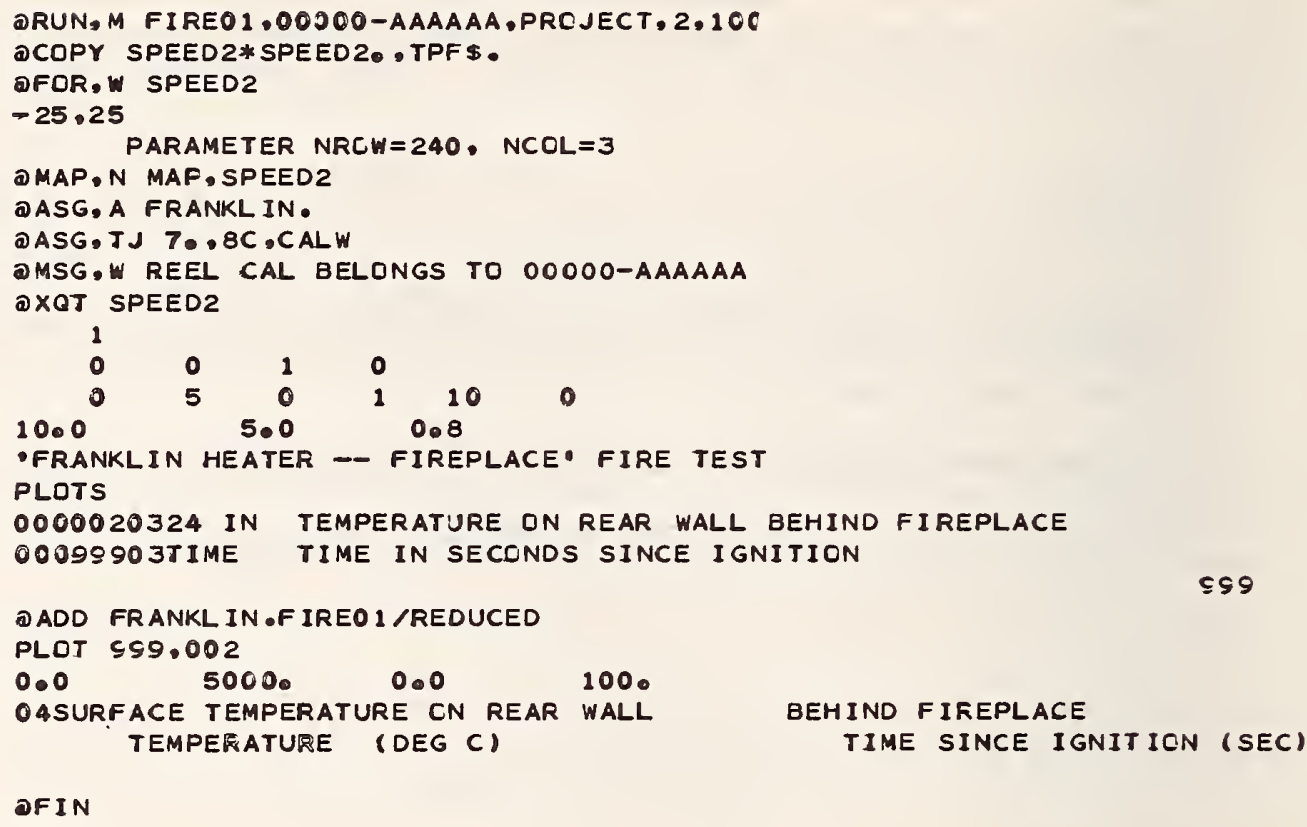

BEHIND FIREPLACE

TIME SINCE IGNITION (SEC) 
If a number of related tests have been processed using SPEED2, with the reduced data stored on mass storage, magnetic tape, punched cards, or the like, the user may wish to compare instruments from several different tests using PLOT2 or PLOT3. This example illustrates the use of PLOT4 to create a new combined set of reduced data that may then be further processed with PLOT2 or PLOT3.

Explanation (numbers refer to lines within the example):

1. Standard UNIVAC 1108 EXEC 8 run initiation card.

2. Copy the mass storage file SPEED2*SPEED2 containing all the subprograms of SPEED2 into the temporary workspace file TPF\$.

3-5. Call the FORTRAN language processor to compile the main program to change the size of the data arrays.

6. Call the @MAP processor (the collector) to collect together the relocatable elements of the subprograms of the SPEED2 system to produce an absolute element for execution.

7. Assign a temporary mass storage file called 10.

8. Assign an existing mass storage file called FRANKLIN.

9. Execute the absolute element SPEED2 created earlier with the amAP processor.

10-11. SPEED2 data cards $A 1$ and $A 2$-- specifies one data set is to be processed using PLOT4.

12. PLOT4 data card Al -- entries specify: do not print the combined data matrix (NPRT=0); and output the combined data matrix to unit 10 -- in this case, a previously assigned mass storage file $(\mathrm{NPNCH}=10)$.

13-14. PLOT4 data cards Bl and B2 -- the title of the experiment.

15-102. PLOT4 data cards B3 -- instrument definition cards for tests $1-4$.

103. PLOT4 data card B4 signals the end of the set of instrument defining cards.

104. PLOT4 data card Cl specifies that the data which follows belongs to test number 1 .

105. Element FIRE01/REDUCED in the mass storage program file FRANKLIN contains the set of reduced data for test number: 1 output by PLOT2. The QADD control statement is used to add into the runstream at this point the entire contents of the element. These are PLOT4 data cards C2.

106-111. Three similar sets of PLOT4 data cards part C for tests 2,3 and 4. 
112. PLOT4 data card C3 specifying the end of the data sets.

113. Standard UNIVAC 1108 EXEC8 run termination card. 
1:

2:

3:

4:

5:

6:

7:

8:

9:

$10:$

11:

$12:$

13:

14:

15:

16:

17:

18:

19:

20:

21:

22:

23:

24:

25:

26:

27:

28:

29:

30 :

31:

32 :

33 :

34:

35 :

36 :

37:

38:

39:

40:

41:

42:

43:

44:

45:

46:

47:

48:

49:

50:
DRUN, M FIRE, CO000-AAAAA, PROJECT, 2, 100

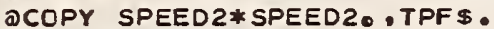

QFOR,W SPEED2

$-25 \cdot 25$

PARAMETER NROW $=200 ; \quad N C O L=90$

\MAP, N MAP, SPEED 2

QASG, T 10 .

QASG, A FRANKLIN。

QXQT SPEED2

$$
\begin{array}{rrrr}
1 & & & \\
0 & 0 & 0 & 1 \\
0 & 10 & &
\end{array}
$$

-FRANKLIN HEATER -- FIREPLACE" FIRE TEST

ALL TESTS

00100103FLUX HEAT FLUX RADJOMETER, REAR WALL $00100002 T C$ REF REFERENCE THEFMOCOUPLE

$00100202 R$ W 46 TEMPERATURE, REAR WALL, $0.46 \mathrm{M}$ UP

$00100302 R W 107$ TEMPERATURE, REAR WALL, $1.07 \mathrm{M}$ UP

00100402RW 168 TEMPERATURE, REAR WALL, $1.68 \mathrm{M}$ UP

00100502 W 229 TEMPERATURE, FEAR WALL, 2.29 M UP

$001006025 W 46$ TEMPERATURE, SIDE WALL, $0.46 \mathrm{M}$ UP

001007025107 TEMPERATURE, SIDE WALL, $1007 \mathrm{M}$ UP

00100802SW 168 TEMPERATURE, SIDE WALL, $1.68 \mathrm{M}$ UP

$001009025 W 229$ TEMPERATURE, SIDE WALL, 2.29 M UP

00101002HEARTH TEMPERATURE, HEARTH CENTER

$00101102 R T$ LEG TEMPERATUEE, BENEATH RIGHT LEG

$00101202 L T$ LEG TEMPERATURE, BENEATH LEFT LEG

$00101302 R$ LEG TEMPERATURE, BENEATH REAR LEG

O0101402R SIDE TEMPERATURE, RIGHT SIDE OF FIREPLACE

$00101502 R$ REAR TEMPERATURE, FIGHT FEAR OF FIREPLACE

O0101602C REAR TEMPERATURE, CENTER REAR OF FIREPLACE

C0101702L REAR TEMPERATURE, LEFT REAR CF FIREPLACE

Oग101802L SIDE TEMPERATURE, LEFT SIDE OF FIREPLACE

ON101902FLOOR TEMPERATURE, FLOOR SURFACE CENTER

001020025 STAK TEMPEFATURE ON SIDE WALL AT STACK

00199901 TIME ELAPSED TIME SINCE IGNITION

$00200103 F L U X$ HEAT FLUX EADIOMETER, REAR WALL

$00200002 T C$ REF REFERENCE THERMOCDUPLE

$0 J 200202 R W 46$ TEMPERATURE, REAR WALL, $0.46 \mathrm{M}$ UP

$00200302 R W 107$ TEMPERATURE, REAF WALL, 1.07 M UP

$0.2200402 R W 168$ TEMPERATURE, REAR WALL, $1.68 \mathrm{M}$ UP

$00200502 R W 229$ TEMPERATUEE, REAFS WALL, 2.29 M UP

$022006025 . W 46$ TEMPERATURE, SIDE WALL, $0.46 \mathrm{M}$ UP

0९2007025W 107 TEMPERATURE, SIDE WALL, 1.07 M UP

002008025W 168 TEMPERATUEE, SIDE WALL, $1.68 \mathrm{M}$ UP

$002009025 W 229$ TEMPERATUFE, SIDE WALL, 2.29 M UP

O0201002HEARTH TEMPERATURE, HEARTH CENTER

$00201102 R T$ LEG TEMPEFATURE, BENEATH RIGHT LEG

$00201202 L T$ LEG TEMPEEATURE, BENEATH LEFT LEG

C $3201302 R$ LEG TEMPERATURE, BENEATH REAR LEG
$W / C M 2$

DEG $C$

DEG $C$

DEG C

DEG C

DEG C

DEG C

DEG C

DEG C

DEG C

DEG C

DEG C

DEG C

DEG C

DEG C

DEG C

DEG $C$

DEG C

DEG C

DEG C

DEG C

SEC

W/CM2

DEG C

DEG C

DEG C

DEG C

DEG C

DEG $C$

DEG C

DEG C

DEG C

DEG $C$

DEG C

DEG C

DEG C 
$51:$

$52:$

$53:$

54:

55:

$56:$

57:

58:

59:

60:

61:

62:

63:

64:

65:

$66:$

67:

68:

69:

70 :

$71:$

$72:$

$73:$

$74:$

75:

76 :

77:

78:

79:

80:

81:

82 :

$33:$

84:

85:

86:

87:

88:

89:

90:

91 :

92:

93:

$94:$

95:

96:

$97:$

98 :

99:

100:
OJ201402R SIDE TEMPERATURE, RIGHT SIDE OF FIREPLACE O0201502R REAR JEMPERATURE, RIGHT REAR OF FIREPLACE O0201602C REAF JEMPERATURE, CENTER REAF OF FIREPLACE O0201702L REAR TEMPEFATUFE, LEFT REAR CF FIREPLACE O.2201802L SIDE TEMPERATUEE, LEFT SIDE CF FIREPLACE O0201902FLCCR TEMPERATURE, FLOOR SURFACE CENTER OD202002S STAK TEMPERATURE ON SIDE WALL AT STACK 00299901 TIME ELAFSED TIME SINCE IGNITICN COS001C3FLUX HEAT FLUX RADICMETER, REAR WALL $00300002 T C$ KEF REFERENCE THEFMOCOUPLE $00300202 \mathbb{1}$ W6 TEMPERATURE, REAR WALL, 0.46 M UP $00300302 \mathrm{WW} 107$ TEMPERATURE, REAR WALL, $1.07 \mathrm{M}$ UP $00300402 \pi W 168$ TEMPERATURE, REAR WALL, $1.68 \mathrm{M}$ UP $00300502 R$ W 229 TEMPERATURE, REAR WALL, 2.29 M UP $003006025 W 46$ TEMPERAATURE, SIDE WALL, $0.46 \mathrm{M}$ UP 003007025 W 107 TEMPERATURE, SIDE WALL, $1.07 \mathrm{M}$ UP 003008025 W 168 TEMPERATUEE, SIDE WALL, $1.68 \mathrm{M}$ UF $003009025 W 229$ TEMPERATURE, SIDE WALL, 2.29 M UP O0301002HEARTH TEMPERATURE, HEAR̄TH CENTEF $00301102 R T$ LEG TEMPERATURE, BENEATH RIGHT LEG $02301202 L T$ LEG TEMPERATURE, BENEATH LEFT LEG $00301302 R$ LEG TEMPEFATURE, BENEATH REAR LEG C $301402 R$ SIDE TEMPERATURE, RIGHT SIDE OF FIREPLACE O0301502R REAR TEMFERATUEE, KIGHT FEAR OF FIREPLACE O $301502 C$ REAR TEMPERATUFE, CENTER FEAF DF FIREPLACE $00301702 L$ REAR TEMPEKATUF̈E, LEFT REAÑ CF FIREPLACE $00301802 L$ SIDE TEMPERATURE, LEFT SIDE CF FIREPLACE $00301902 F L C O K$ TEMFERATURE, FLODF SURFACE CENTER O 33020025 STAK TEMPERATURE ON SIDE WALL AT STACK 00399901 TIME ELAPSED TIME SINCE IGNITICN OO400103FLUX HEAT FLUX RADIOMETER, REAR WALL 00400002TC REF REFEKENCE THEFMOCOUPLE $03400202 R W 46$ TEMPEFATUEE, SEAF WALL, 0.46 M UP $00400302 R$ W 1 C7 TEMPEFATURE, REAR WALL, 1.07 M UP $00400402 R W 168$ TEMPEFATUEE, REAF WALL, 1.68 M UP $00400502 R W 229$ TEMPERATURE, FEAR WALL, $2.29 \mathrm{M}$ UP CC400602SW 46 TEMPERATURE, SIDE WALL, 0.46 M UP \$0400702SW 107 TEMPEF.ATURE, SIDE WALL, 1.07 M UP 00400802SW 168 TEMPERATURE, SIDE WALL, 1.68 M UP $004009025 W 229$ TEMPERATURE, SIDE WALL, 2.29 M UP O0401002HEARTH TEMPERATURE, HEARTH CENTER OO401102RT LEG TEMPERATURE, BENEATH RIGHT LEG 604012C2LT LEG TEMPERATURE, BENEATH LEFT LEG $00401302 R$ LEG TEMPERATURE, BENEATH EEAR LEG O9401402R SIDE TEMPERATURE, RIGHT SIDE OF FIREPLACE $00401502 R$ REAR TEMPERATUFE, FIGHT REAR OF FIEEPLACE $00401602 C$ REAR TEMPERATUKE, CENTER REAR OF FIREPLACE $03401702 L$ REAR TEMPERATUEE, LEFT FEAR CF FIREPLACE CC401802L SIDE TEMPERATURE, LEFT SIDE CF FIREPLACE 00401902FLCOR TEMPERATURE. FLOCR SUPFACE CENTER
DEG C DEG $C$ DEG C DEG C DEG C DEG $C$ DEG C SEC

$W / C M 2$ DEG C DEG C DEG C DEG C DEG C DEG C DEG C DEG C DEG $C$ DEG C DEG C DEG C DEG C DEG C DEG C DEG C DEG C DEG C DEG C DEG C SEC W/CM2 DEG C DEG C DEG C DEG C DEG C DEG C DEG C DEG C DEG C DEG C DEG C DEG C DEG C DEG C DEG C DEG C DEG C DEG C DEG C 


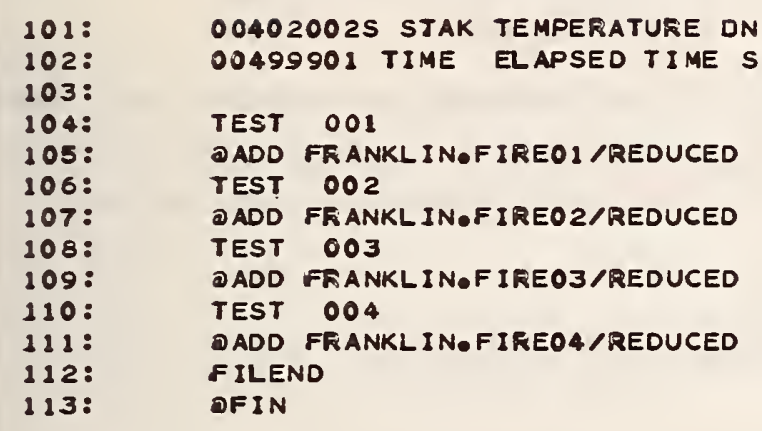

DEG C

SEC 
The reduction of data using SPEED2 can, of course, be considerably more complex than presented in example 2. In this example, subprogram CoNv is used with FORTRAN code to provide complicated data reduction and analysis.

Explanation (numbers refer to lines within the example):

1. Standard UNIVAC 1108 EXEC 8 run initiation card.

2. Copy the mass storage file SPEED2*SPEED2 containing all the subprograms of SPEED2 into the temporary workspace file TPF\$.

3-5. Call the FORTRAN language processor to compile the main program SPEED2, changing the size of the data arrays to NROW=51 and $\mathrm{NCOL}=36$ to allow up to 36 columns and 51 rows of data (note that one extra column is needed by SPEED2 over and above the number columns needed by the user).

6-7. Call the FORTRAN language processor to compile the subprogram CONV to provide FORTRAN changes for data reduction. Insert the FORTRAN code that follows in the runstream after line 17.

8-9. Call subprogram FIND to determine the location of instrument number 126. The subscript (column) of the array REED containing the readings from instrument number 126 is returned in the variable Ll26.

10. Establish the variable MAXROW as the maximum number of readings from instrument number 126 . This variable will be used throughout as the maximum number of readings for all instruments.

11-14. Convert the readings of instrument number 126 with a simple FORTRAN do loop.

15-34. Similar conversions for other instruments.

35-36. A more complicated conversion on several instruments by using an additional FORTRAN DO loop with instruments numbered 129 through 134 .

37-43. Convert the millivolt output for these instruments into meaningful units.

44-46. Create new instruments (which must be defined in instrument cards) numbered 329 through 334 and set the maximum number of readings for these instruments by letting MAXR(i) equal MAXROW for these instruments.

47-54. Define the readings for these user created instruments as conversions of readings from instruments scanned by the data acquisition system.

55-97. Similar conversions for other instruments.

98-121. Create four new instruments as different conversions from a single instrument. 
Call the amAP processor (the collector) to collect together the relocatable elements of the subprograms of the SPEED2 system to produce an absolute element for execution.

123. Assign a temporary mass storage file called 10.

124. Execute the absolute element SPEED2 created earlier with the @MAP processor.

125-130. SPEED2 data cards Al and A2, PLOT2 data cards A1, A2, B1 and B2.

131-165. PLOT2 data cards B3 -- instrument definition cards for instruments to be processed.

166. PLOT2 data card B4 signals the end of the set of instrument defining cards.

167-170. PLOT2 data cards B5 specifying conversion coefficients for instrument types $1,2,3$, and 4 .

171. PLOT2 data card B6 signals the end of the set of coefficient cards.

172. Element TESTI7 in the mass storage program file RAN-DATA contains raw data images in the format of the data acquisition system prepared by using PLOTl or PLOT2. The GADD control statement is used to add into the runstream at this point the entire contents of the element. These are PLOT2 data cards part $\mathrm{E}$.

173-216. Several sets of four cards to generate separate printer plots.

217. A blank card signals the end of the plot generating cards.

218. Copy the temporary mass storage file named 10 containing the reduced data output by SPEED2 (NPNCH=10) into permanent storage in the element named TESTI7 within the mass storage program file named REDUCED-DATA.

219. Standard UNIVAC 1108 EXEC 8 run termination card. 


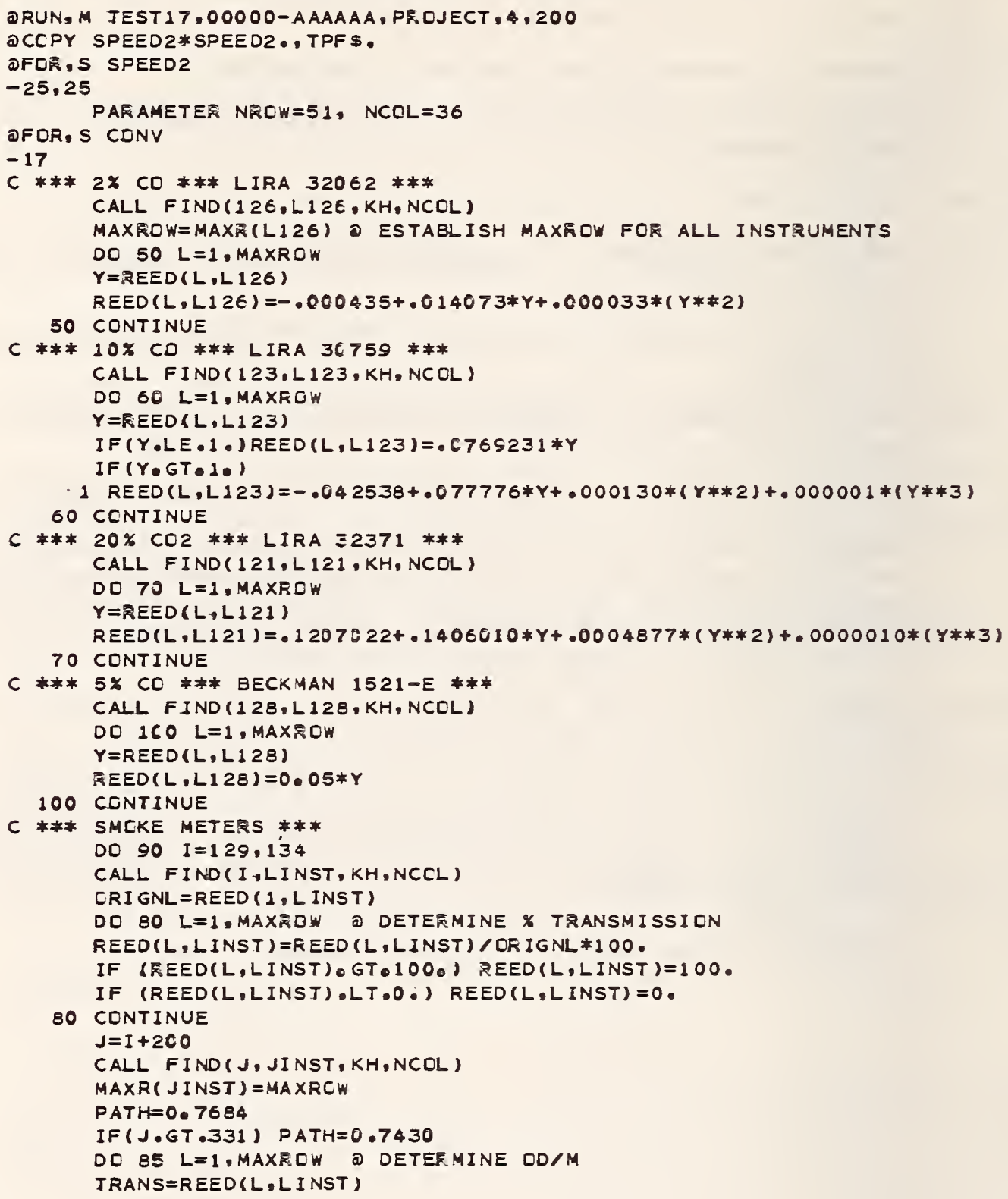


IF (TRANS.LT.0.001) TRANS $=0.001$

52:

REED(L, JINST $)=($ ALOG $10(1000 / T$ TANS $)$ )/PATH

85 CONTINUE

90 CONTINUE

C *** 02 READINGS ***

DO $130 \quad K=125,127$

$I=K$

IF(K॰EQ.126) I=124 @ DO CHANNEL S $124,125,127$

CALL FINO(I,LINST,KH,NCOL)

ORI GNL $=$ REED $(1$, LINST $)$

DO $120 . L=1$, MAXROW @ COMPUTE \& CONCENTRATION

REED (L.LINST) $=$ REED $(L$, LINST $) * 21, / O R I G N L$

120 CONTINUE

$J=I+200$

CALL FIND (J,JINST, KH,NCOL)

MAXR ( JINST) = MAXEOW

DO 125 L=1. MAXROW a COMPUTE (CHANGE IN * CONCENTRATION) = (

REED(L.JINST) $=2100-R E E D(L, L I N S T)$

125 CONTINUE

c

130 CONTINUE

C *** BURN RATE OF CRIB (PERCENT LOSS) ***

c

c

c

c

C

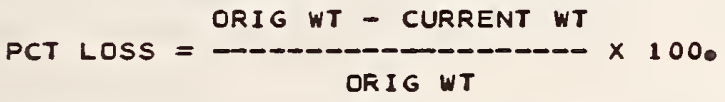

CALL FIND (135.L135,KH,NCOL)

CALL FIND(305, L $305, \mathrm{KH}, \mathrm{NCOL})$

$\operatorname{MAXR}(L 305)=$ MAXROW

CRI GNL $=$ REED $(1, L 135)$

DO $190 \mathrm{~L}=1$, MAXROW

190 RED $(L, L 305)=($ DRI GNL-त̃EED $(L, L 135)) / O R I G N L * 100$. $c$

C *** BURN RATE OF CRIB (PERCENT LOSS PER SECCND) ***

c

PCT LOSS CHANGE IN PCT LOSS OVER EACH 10 SEC INTERVAL

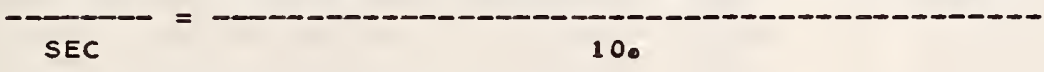

CALL FIND ( $306, L 306, \mathrm{KH}, \mathrm{NCOL})$

$\operatorname{MAXR}(L 306)=M A X R O W$

ORI GNL $=$ REED $(1, L 135)$

REED $(1, L 306)=0$ :

DO 200 L $=2$, MAXROW

$L M 1=L-1$

200 REED $(L, L 306)=(R E E D(L, L 305)-R E E D(L M 1, L 305)) / 10$ :

C

C *** BURN RATE OF CRIB (GRAMS/SEC) *** 
101:

102:

$105:$

$104:$

105:

$106:$

107:

108:

109:

110:

111:

112:

113:

$114:$

$115:$

116:

$117:$

$118:$

119:

120 :

121:

$122:$

$123:$

$124:$

$125:$

126 :

$127:$

128 :

$129:$

130 :

131:

132 :

$133:$

$134:$

$135:$

136:

$137:$

$138:$

$139:$

140:

141:

142:

$143:$

$144:$

145:

146:

147:

148:

$149:$

150 :
CALL FIND (307,L $307, K H, N C O L)$

CALL FIND $(308, L 308, K H, N C C L)$

CALL FIND $(309, L 305, K H, N C O L)$

CALL FIND $(310, L 310, \mathrm{KH}, \mathrm{NCOL})$

MAXR (L 307) = MAXRCW

MAXR $(L 308)=$ MAXEOW

MAXR (L $30 S)=$ MAXROW

MAXR $(L 310)=$ MAXR OW

C CONVERT LOAD CELL READINGS FROM PCUNDS TO GRAMS

DO $210 L=1$, MAXROW

210 REED(L,L 307$)=R E E D(L, L 135) * 453.6$

C DETERMINE BUFN RATE (G/S) CVEF A 10-SEC, 30-SEC, 60-SEC INTERVAL DO $220 \mathrm{~L}=2$, MAXRDW

$L$. $11=L-1$

220 KEED $(L, L 308)=($ XEED $(L M 1, L 307)-\overline{X E E D}(L, L 307)) / 10$.
DO 230 L=4, MAXFOW

$L M B=L-3$

230

REED $(L, L 309)=(R E E D(L$ 13,L 307$)-R E E D(L, L 397)) / 30$. DO 240 L=7, MAXROW

$L M G=L-6$

240 KEED $(L, L 310)=($ KEED $(L M 6, L 307)-$ KEED $(L, L 307)) / 60$. D MAP, S MAP, SPEED2

D $A S G, T 10$.

๑XQT SPEED2$$
\begin{array}{crc}
1 & & \\
0 & 1 & c \\
3 & -1 & 0 \\
0 & 1 & 10 \\
* * * & \multicolumn{2}{c}{\text { MOBILE }}
\end{array}
$$

$\begin{array}{lr}00007102 T C & 1 \\ 00007202 T C & 2 \\ 00007302 T C & 23 \\ 00007402 T C & 37 \\ 00012004 R A O & 1 \\ 00012502 H S M & 4 \\ 00013002 H S M & 5 \\ 00013162 H S M & 6 \\ 00013202 H S M & 8 \\ C 0013302 H S M & 9 \\ 00013402 H S M & 10 \\ 00032902 H S M & 4 \\ 00033002 H S M & 5 \\ 00033102 H S M & 6 \\ 00033202 H S M & 8 \\ 00033302 H S M & 9 \\ 00033402 H S M & 1 C \\ 00012302 C O & 13 \\ 00012102 C O 2 & 14 \\ 0001240202 & 15\end{array}$

D LOAD CELL READINGS IN GRAMS

10 SECOND INTEX̃VAL

D 60 SECOND INTER VAL$$
\text { (axaT }
$$

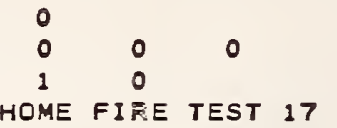

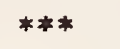

1

2

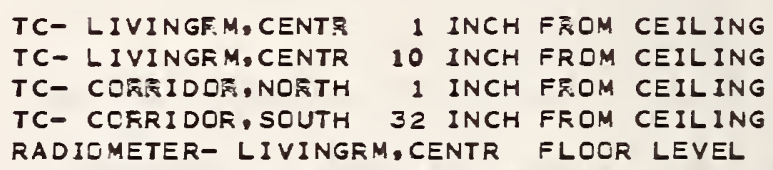




$\begin{array}{lll}151: & 0001250202 & 24 \\ 152: & 00012602 C 0 & 16 \\ 153: & 0001270202 & 17 \\ 154: & 00012802 C 0 & 25 \\ 155: & 0003240202 & 15 \\ 156: & 0003250202 & 24 \\ 157: & 0003270202 \text { 17 } \\ 158: & 00013503 L \text { CELL } \\ 159: & 00030502 \% \text { LOSS } \\ 160: & 00030602 \times / S E C \\ 161: & 00030702 \text { GRAMS } \\ 162: & 000308026 / S 10 \\ 163: & 00030902 \text { G /S } 30 \\ 164: & 000310026 / S 60 \\ 165: & 00000001 \text { TIME }\end{array}$

$166:$

$167:$

168:

$169:$

170:

171:

$172:$

173:

174:

175:

$176:$

177 :

178:

$179:$

180:

181:

$182:$

183:

$184:$

185 :

186:

187:

188:

$189:$

190:

191:

192:

193:

194:

195:

196:

197:

198:

199:

$200:$

.0940

2.69

PLUT $0000 \quad 0120$

D2 - DOOR OF LIVINGRM AT I IN ABOVE FLOCR

CO - SOUTH CORRIDOR AT 5 FT ABCVE FLOCR

D2 - SOUTH CORAIDOE AT 5 FT ABCVE FLOOR

CO - SOUTH CORRIDOF AT 3 FT ABOVE FLOOR

02 - CENTER CF LIVINGRM, 5 FT ABV FLR (CHG IN CONC)

02 - DOOR OF LIVINGRM, I IN ABV FLR (CHG IN CONC)

02 - SUUTH COKRIDOR, 5 FT ABV FLF. (CHG IN CONC)

LOAD CELL (POUNDS)

BURN RATE IN PERCENT LOSS

BURN RATE IN PEECENT LOSS PER SECOND

LOAD CELL (GRAMS)

BURN RATE IN GFAMS PEF SECOND (1O-SEC INTERVAL)

BURN RATE IN GRAMS PER SECOND (30-SEC INTERVAL)

BURN RATE IN GRAMS PER SECCND (6O-SEC INTERVAL)

TIME IN SECONDS AFTER IGNITION

660.0

$\triangle A D D R A W-D A T A \bullet T E S T 17$

PLOT OC00 0071007200730074

0.01250 .1000 . 0.0

THERMOCOUPLES- LIV F.M CENTER (1.10 IN), CORR N ( I IN), COF.F S ( 32 IN) DEGREES C

SECONDS AFTER IGNITICN

0.1250 .0 . 5.

RADIOMETER- CENTER OF LIVING ROCM, FLOOR LEVEL

WATTS PER SQUARE CM

PLOT COOO 03290330.0331

O. 1250 . O० 7.5

SMOKE METERS- CORRIDOR, NDRTH $(2,4,6$ FT ABCVE FLCOR)

OPTICAL DENSITY PEE METEE SECONDS AFTER IGNITIEN

FLOT $0000 \quad 03320333 \quad 0334$

0. 1250. 0.705

SMOKE METERS- CORRIDCR, SOUTH (2,4,6 FT ABOVE FLOOR) OPTICAL DENSITY PER METER

PLOT 0000012401250127

0.250 . 1250

D2- LIV RM CENTER (5 FT), LIV FM DOOR ( 1 IN), CORR SLUTH (5 FT) PERCENT GAS IN THE ATMOSPHERE SECONDS AFTER IGNITICN

PLOT OOCO 01230121

0.1250 .20$.

$10 \% \mathrm{CO}, 20 \% \mathrm{CD} 2-$ LIVING F.OOM CENTER (5 FT ABOVE FLCOR)

PEFCENT GAS IN THE ATMOSPHEEE SECONDS AFTER IGNITICN

PLOT OCCO 01260128

2\% CO (5 FT UP), $5 \%$ CO (3 FT UP) - CCIFRIDOF SCUTH PERCENT' GAS IN THE ATMOSPHERE

SECONDS AFTER I GNITICN 


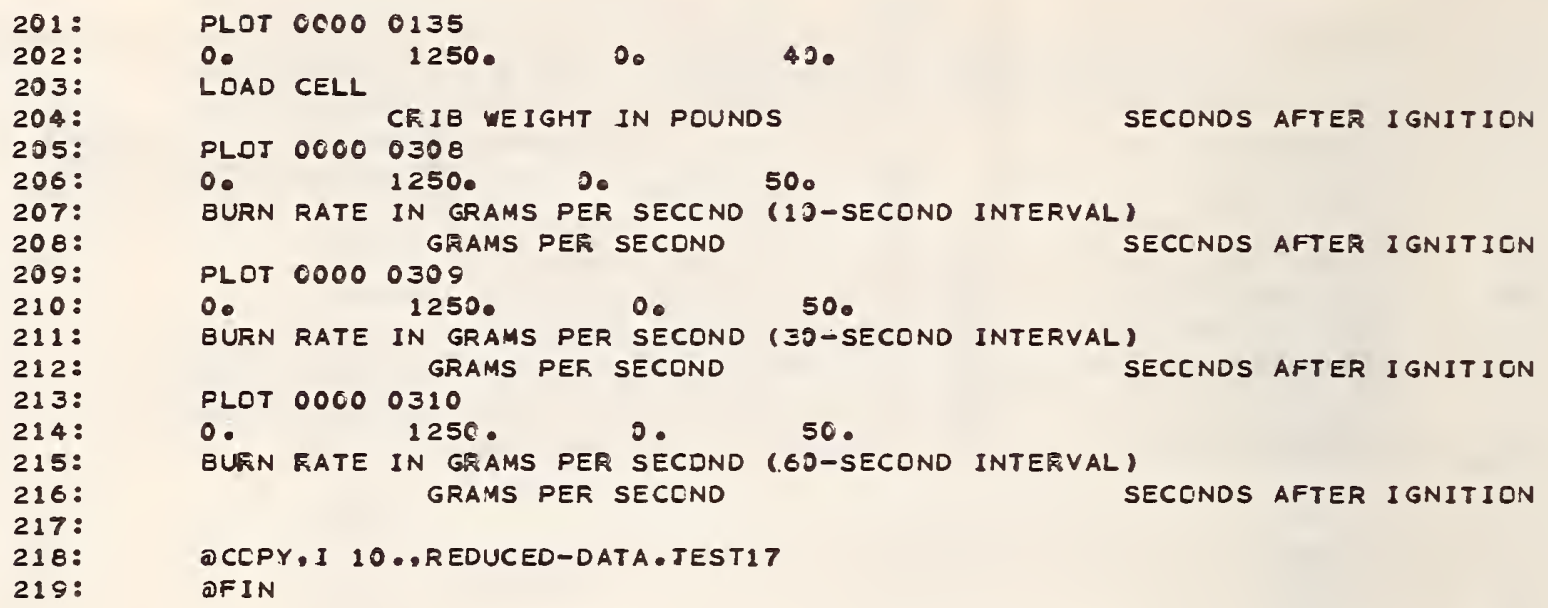


NBS-114A (REV. 9-78)

U.S. DEPT. OF COMM.

BIBLIOGRAPHIC DATA SHEET

1. PUBLICATION OR REPORT NO.

NBS Tech Note 1108

4. TITLE AND SUBTITLE

SPEED2, A Computer Program for the Reduction of Data

from Automatic Data Acquisition Systems

September 1979

C. Perforahing Organizatión Code

8. Performing Organ. Report No.

Richard D. Peacock and John M. Smith

19. Profectrask/fork Unit No.

9. PERFORMING ORGANIZATION NAME AND ADDRESS

NATIONAL BUREAU OF STANDARDS

DEPARTMENT OF COMMERCE

11. Contract/Grant No.

WASHINGTON, DC 20234

12. SPONSORING ORGANIZATION NAME AND COMPLETE ADDRESS (Streol, City, State, ZIP)

13. Type of Report \& Period Covered Final

14. Sormorind Agericy Coge

15. SUPPLEMENTARY NOTES

X Document describes a computer program; SF-185, FIPS Software Summary, is attached.

16. ABSTRACT (A 200-word or less factual summary of most significant information. If document includes a significant bibliography or literature survey, mention it here.)

The voluminous amount of data that can be collected by automatic data acquisition systems requires the use of a digital computer for the reduction of data. A general purpose computer program for the reduction of data collected by automatic data acquisition systems is presented. The program is written with the ability to accept data from a number of different data acquisition systems, with the ability to check the correctness of data included. Through the use of FORTRAN computer programming, the data can be converted to meaningful scientific and engineering units. The data can then be presented in tabular, printer plot or ink pen plot form.

The program is documented, and detailed instructions for 'its use, with examples, are presented. The use of SPEED2 requires some knowledge of FORTRAN programming language and the executive control language for the computer system in use.

17. KEY WORDS (six to twelve entries; alphabetical order; capitalize only the first letter of the first key word unless a proper name; separated by semicolons)

Computer program; data reduction; data acquisition systems; plotting . 

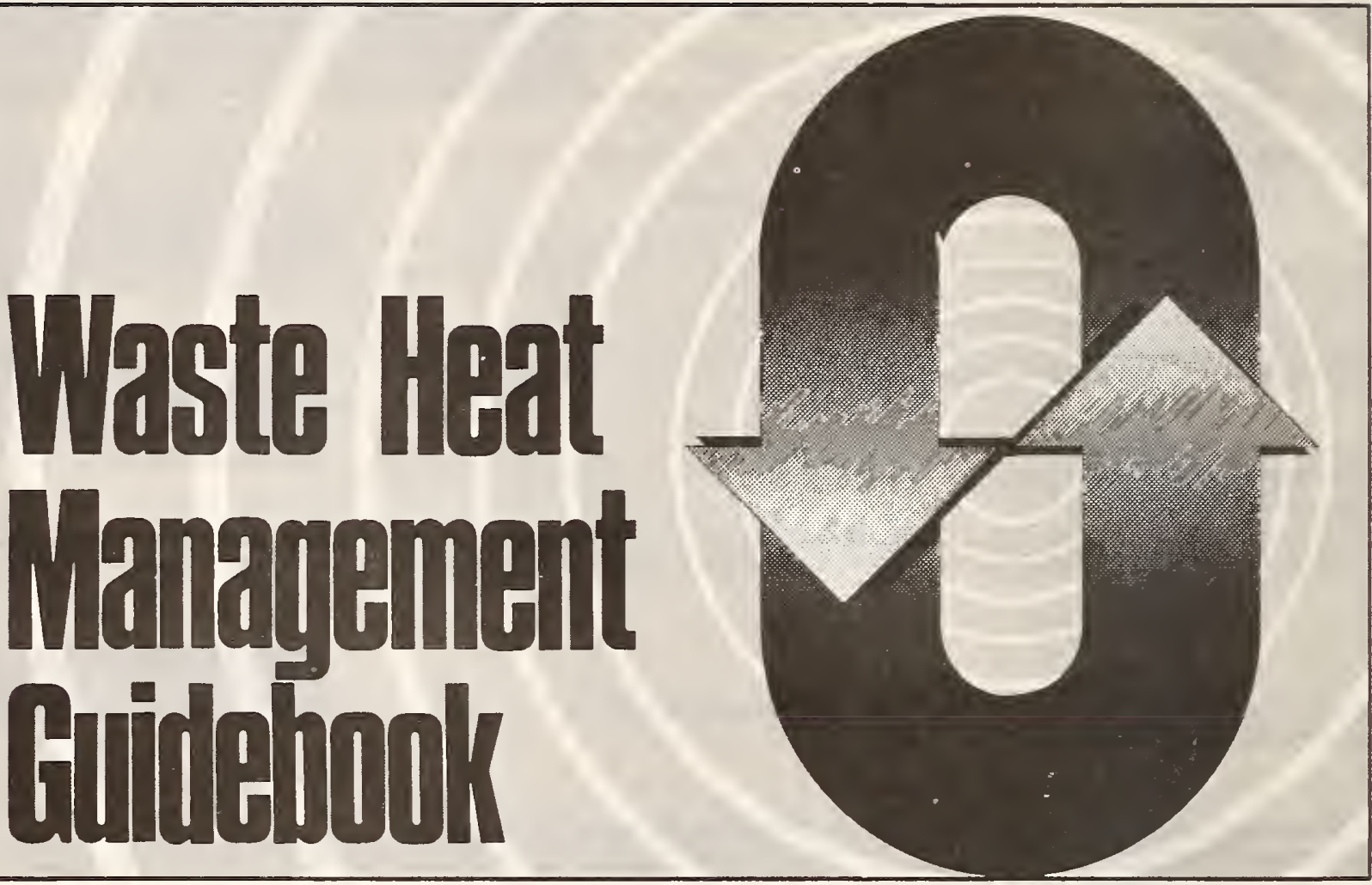

A typical plant can save about 20 percent of its fuel-just by installing waste heat recovery equipment. But with so much equipment on the market, how do you decide what's right for you?

Find the answers to your problems in the Waste Heat Management Guidebook, a new handbook from the Commerce Department's National Bureau of Standards and the Federal Energy Administration.

The Waste Heat Management Guidebook is designed to help you, the cost-conscious engineer or manager, learn how to capture and recycle heat that is normally lost to the environment during industrial and commercial processes.

The heart of the guidebook is 14 case studies of companies that have recently installed waste heat recovery systems and profited. One of these applications may be right for you, but even if it doesn't fit exactly, you'll find helpful approaches to solving many waste heat recovery problems.
In addition to case studies, the guidebook contains information on:

- sources and uses of waste heat

- determining waste heat requirements

- economics of waste heat recovery

- commercial options in waste heat recovery equipment

- instrumentation

- engineering data for waste heat recovery

- assistance for designing and installing waste heat systems

To order your copy of the Waste Heat Management Guidebook, send $\$ 2.75$ per copy (check or money order) to Superintendent of Documents, U.S. Government Printing Office, Washington, D.C. 20402. A discount of 25 percent is given on orders of 100 copies or more mailed to one address.

The Waste Heat Management Guidebook is part of the EPIC industrial energy management program aimed at helping industry and commerce adjust to the increased cost and shortage of energy. 


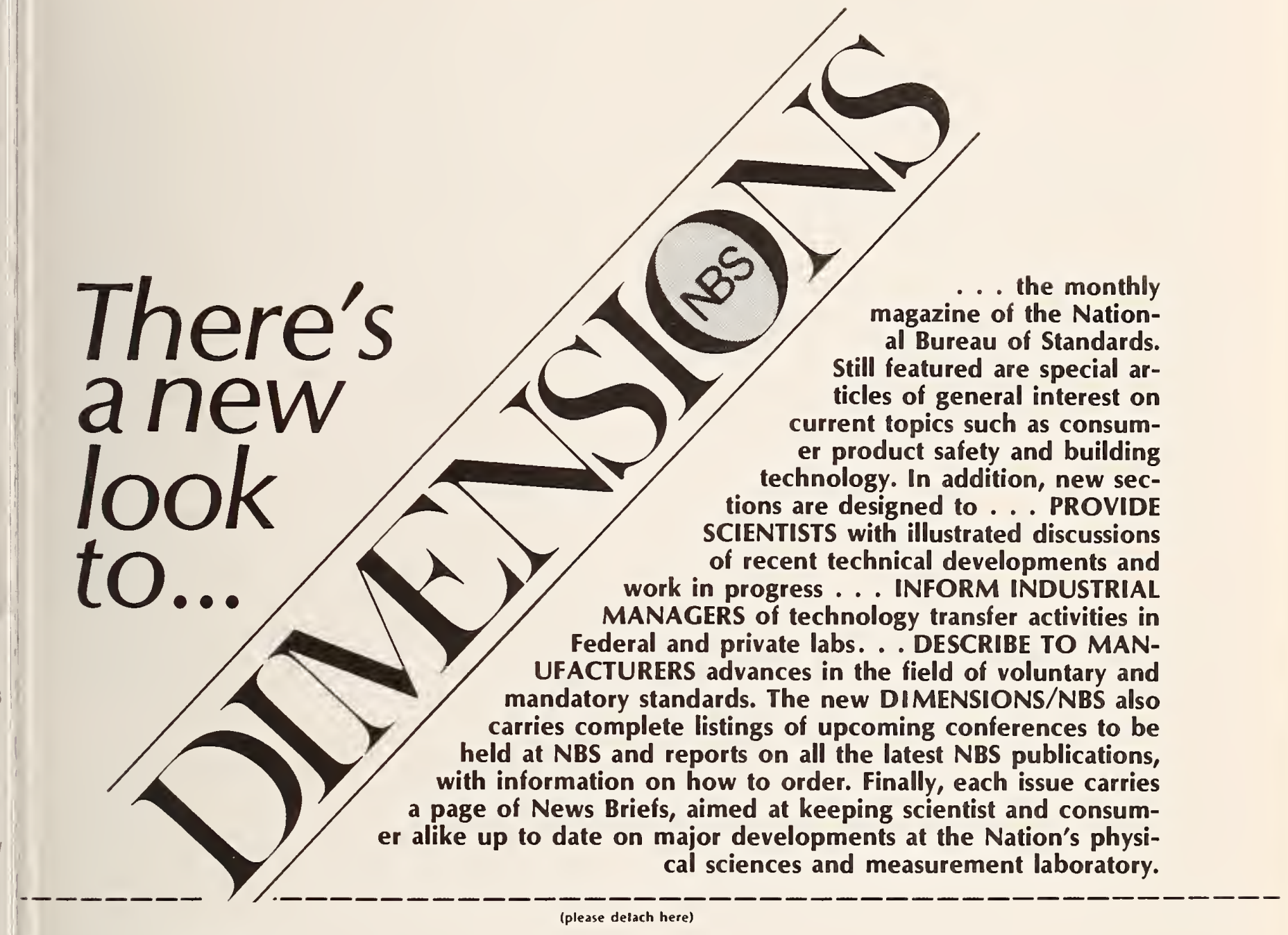

\section{SUBSCRIPTION ORDER FORM}

Enter my Subscription To DIMENSIONS/NBS at $\$ 11.00$. Add $\$ 2.75$ for foreign mailing. No additional postage is required for mailing within the United States or its possessions. Domestic remittances should be made either by postal money order, express money order, or check. Foreign remittances should be made either by international money order, draft on an American bank, or by UNESCO coupons.

Send Subscription to:

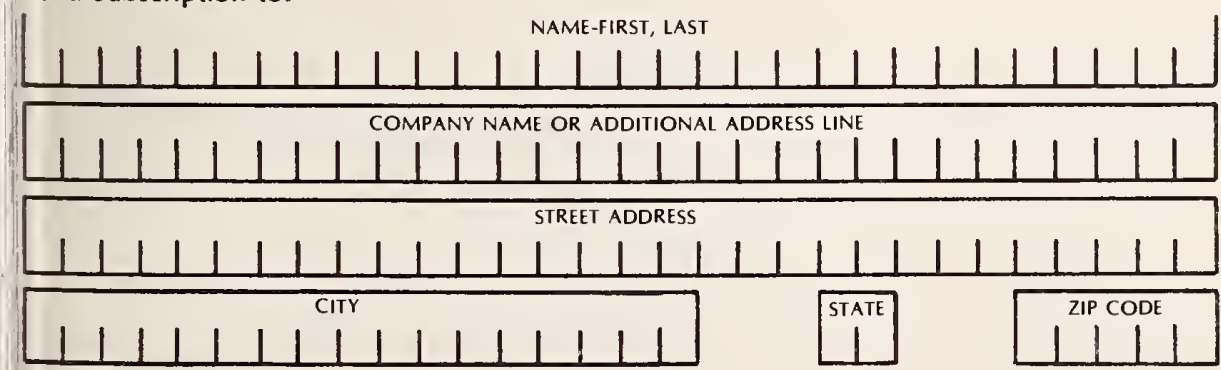

Remittance Enclosed (Make checks payable to Superintendent of Documents)

Charge to my Deposit Account No.

MAIL ORDER FORM TO: Superintendent of Documents Government Printing Office Washington, D.C. 20402 



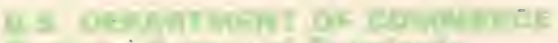

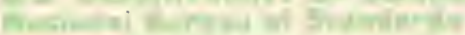

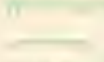

ir non

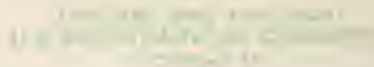

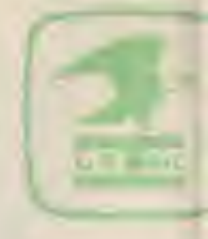

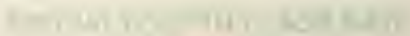

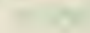

